Sous la direction de

Hoai Huong Aubert-Nguyen et Michel Espagne

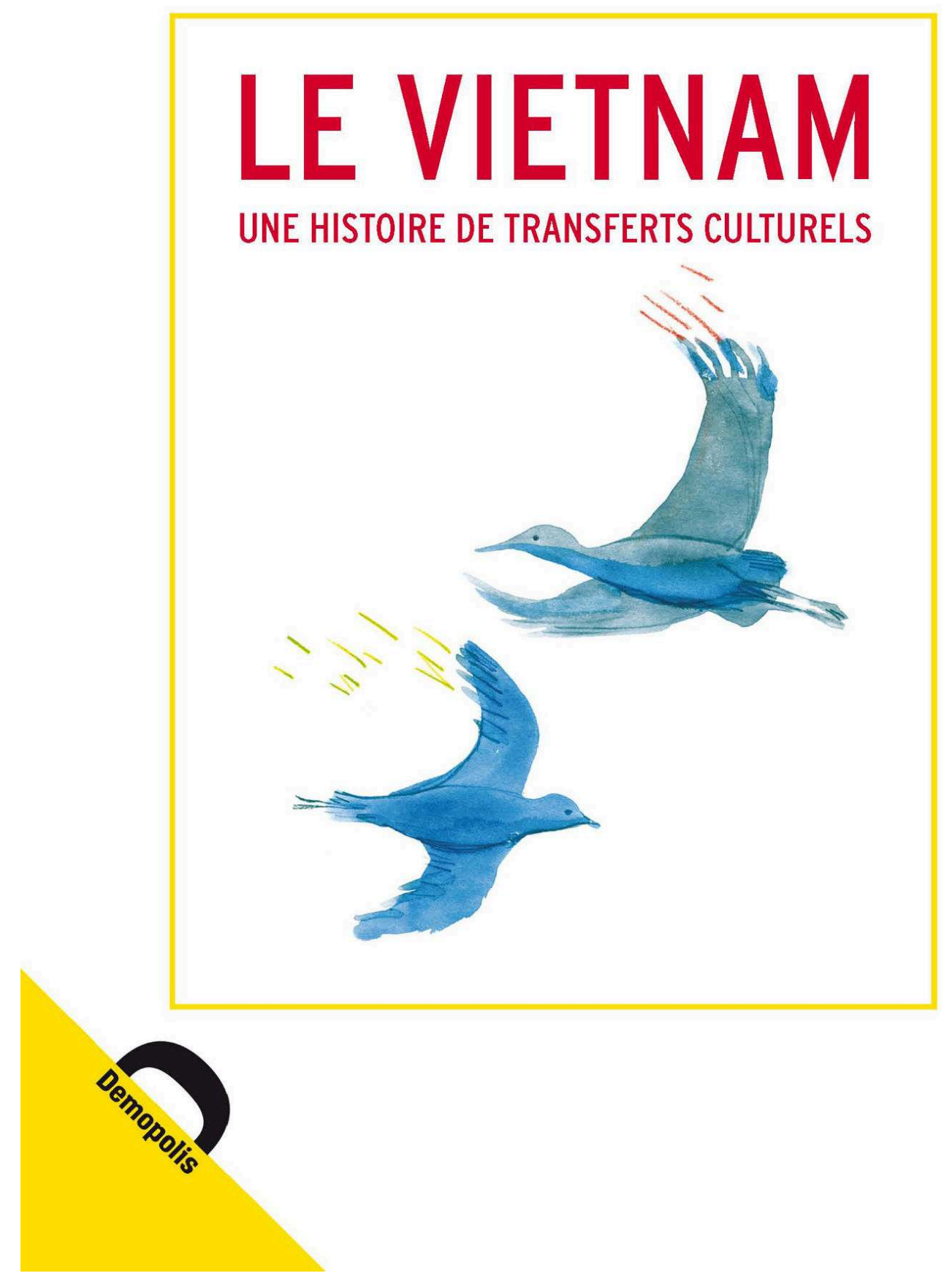




\section{Le Vietnam}

Une histoire de transferts culturels

Hoai Huong Aubert-Nguyen et Michel Espagne (dir.)

DOI : 10.4000/books.demopolis.461

Éditeur : Demopolis

Année d'édition : 2015

Date de mise en ligne : 30 juin 2016

Collection : Quaero

ISBN électronique : 9782354571146

\section{Doneratition}

http://books.openedition.org

Édition imprimée

ISBN : 9782354570774

Nombre de pages : 324

\section{Référence électronique}

AUBERT-NGUYEN, Hoai Huong (dir.) ; ESPAGNE, Michel (dir.). Le Vietnam : Une histoire de transferts culturels. Nouvelle édition [en ligne]. Paris : Demopolis, 2015 (généré le 10 décembre 2020). Disponible sur Internet : <http://books.openedition.org/demopolis/461>. ISBN : 9782354571146 . DOI : https:// doi.org/10.4000/books. demopolis.461.

(c) Demopolis, 2015

Conditions d'utilisation:

http://www.openedition.org/6540 


\section{Sous la direction de \\ Hoai Huong Aubert-Nguyen et Michel Espagne}

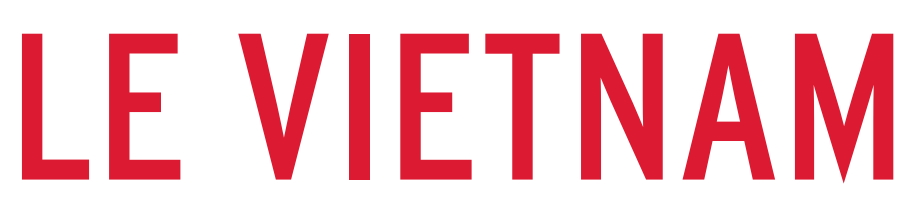

UNE HISTOIRE DE TRANSFERTS CULTURELS

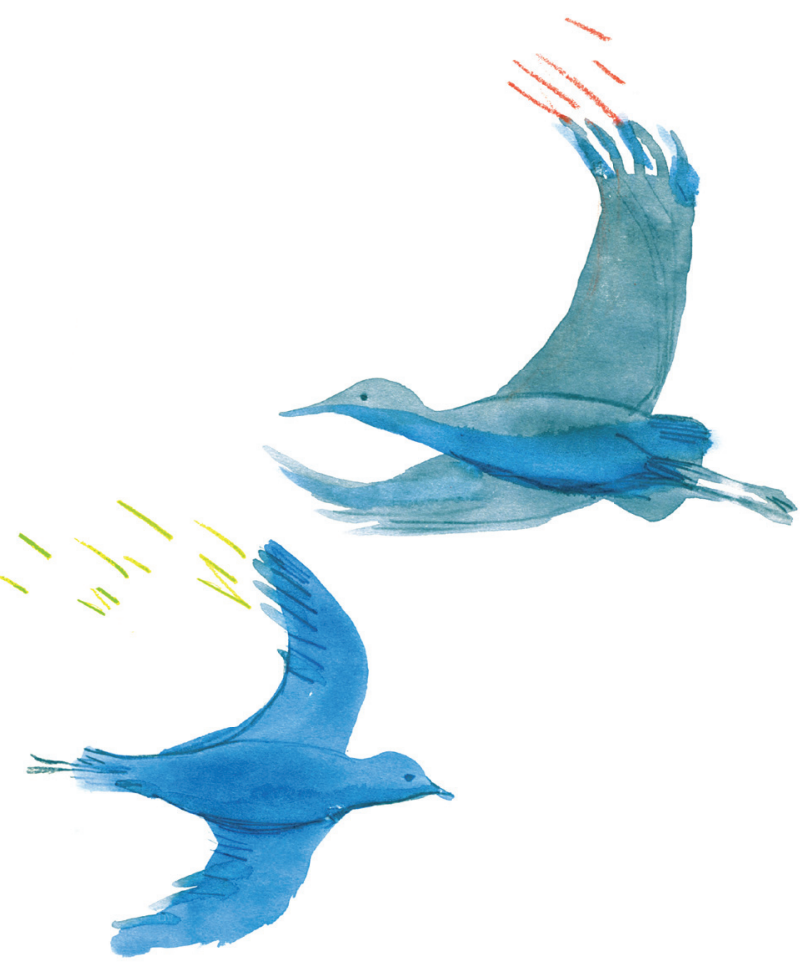






\section{LE VIETNAM}

UNE HISTOIRE DE TRANSFERTS CULTURELS 


\section{«QUAERO »}

Collection dirigée par Jean-Christophe Tamisier

Barbara Cassin et Danièle Wozny (dir.),

Les intraduisibles du patrimoine en Afrique subshaharienne.

Hosham Dawod (dir.),

La constante "Tribu », variations arabo-musulmanes.

Christian Ehrenfreund et Jean-Philippe Schreiber (dir.),

Les marranismes. De la religiosité cachée à la société ouverte.

Alexandre Fontaine,

Aux heures suisses de l'école républicaine.

Christian Ghasarian,

Rapa. Île du bout du monde, ile dans le monde.

Charles-Édouard Niveleau (dir.),

Vers une philosophie scientifique. Le programme de Brentano.

Pascale Rabault-Feuerhahn (dir.),

Théories intercontinentales. Voyages du comparatisme postcolonial.

Michèle-H. Salamagne et Patrick Thominet (dir.),

Accompagner. Trente ans de soins palliatifs en France.

Cet ouvrage a été publié avec le soutien du laboratoire d'excellence TransferS (programme Investissements d'avenir

ANR-10-IDEX-0001-02 PSL* et ANR-10-LABX-0099).

(C) Illustration de couverture: Valérie Linder

(C) Éditions Demopolis, 2015

4 , rue Scipion

75005 Paris

www.demopolis.fr

ISBN : 978-2-35457-077-4 
Sous la direction de

HOAI HUONG AUBERT-NGUYEN

et MICHEL ESPAGNE

\section{LE VIETNAM}

UNE HISTOIRE DE TRANSFERTS CULTURELS 
Le présent volume rassemble les contributions présentées à l'occasion d'un colloque qui s'est tenu à l'École normale supérieure et à la Bibliothèque nationale de France du 4 au 6 juin 2014. Cette rencontre s'inscrivait dans le cadre de l'année FranceVietnam organisée par l'Institut français.

Il convient de remercier ici l'ENS, le labex TransferS, l'IDEX PSL et la BnF qui ont accueilli cette manifestation, ainsi que l'UVSQ dont plusieurs membres ont activement participé à sa préparation. Merci à Valérie Linder pour l'illustration de couverture. 


\title{
Les auteurs
}

\author{
NADINE ANDRÉ-PALLOIS (INALCO) \\ JULIE ASSIER (université de Cergy-Pontoise) \\ HoAI Huong AUBERT-NGUYEN (université de Versailles-St-Quentin) \\ PierRe BROCHEUX (université Paris-Diderot) \\ CAM ThI DOAN (INALCO) \\ MICHEL ESPAGNE (CNRS/ENS) \\ JoËLLE GARCIA (BnF, département des Arts du spectacle/BULAC)
}

DenIS GAZQUEZ (BnF, département Philosophie, Histoire, Sciences de l'homme)

JÉrome GIDOIN (université Paris-Descartes)

ANDREW HARDY (EFEO)

Tuân HOANG VAN (université de Versailles-St-Quentin)

Olivier LOISEAUX (BnF, département des Cartes et Plans)

Pierre-Yves MANGUIN (EFEO)

LAURENCE MONNAIS (université de Montréal)

GIANG Huong NGUYEN (BnF, département Littérature et Art)

Phuong Ngoc NGUYEN (université Aix-Marseille)

Thuy Phuong NGUYEN (université Paris-Descartes)

Veronica NTOUMOS (Paris IV Sorbonne/Université libre de Bruxelles/ aspirante FNRS)

Philippe PAPIN (EPHE)

Alain RUSCIO (CID Vietnam)

THANH VÂN TON THAT (université Paris-Est-Créteil) 

AVANT-PROPOS 

$\mathrm{I}$ existe des représentations idylliques et oniriques du Vietnam transmises par des romans comme L'Amant de Marguerite Duras ou par des films qui illustrent tantôt une société indochinoise raffinée et menacée de disparition dans Indochine, tantôt la sensualité de la vie quotidienne dans l'Odeur de la papaye verte. Le Vietnam s'identifie alors à une suite de goûts, de senteurs, de paysages, à des végétations inconnues sous la chaleur écrasante, aux grandioses paysages de la baie d'Ha Long ou du cours du Mékong. Pourtant, le pays s'est aussi et avant tout confondu dans la conscience et l'imaginaire de beaucoup d'Européens, et plus particulièrement de Français, avec l'histoire de deux guerres et de leurs conséquences.

La première est associée aux images de Dien Bien Phu ou du bagne de Poulo Condor et de ses cages à tigre, à celle de l'infirmière Geneviève de Galard ou du commissaire politique Georges Boudarel. Elle est souvent représentée à travers le prisme de deux conceptions opposées. L'une met en lumière les combats du Vietminh, dont les partisans apparaissent comme des héros de l'anticolonialisme et de la révolution sociale cette vision occultant quelquefois des dérives inhumaines, notamment au cours de la révolution agraire. L'autre repose sur la nostalgie d'une époque magnifiée: celle des enseignants français contribuant à la démocratisation et à la diversification de la culture en Indochine, celle des gloires et de l'honneur militaires, qui transparaissent dans les films de Pierre Schoendoerffer, où la guerre d'Indochine est perçue in fine comme un combat antitotalitaire - vision qui ne peut s'affranchir d'un soupçon d'indulgence vis-à-vis de la féodalité annamite et du colonialisme. Ces deux conceptions sont évidemment inconciliables.

Pour la guerre américaine, on pense à une petite fille brûlée par le napalm qui court sur une route de campagne, au chef de la police de Saigon exécutant un combattant vietcong, à la destruction de Hué et aux 
monstruosités provoquées par l'agent orange, ou plus tard aux derniers officiels américains quittant Saigon par le toit de l'ambassade puis aux boat people errant de Hong-Kong à l'Australie. Du côté des figures vietnamiennes, il y a l'image un peu estompée de l'empereur Bao Dai, celle de Thich Quang Duc s'immolant par le feu, de Ngo Dinh Diem assassiné puis celle de Ho Chi Minh, témoin asiatique du congrès de Tours, et de son général et stratège, enseignant dans le civil l'histoire de France, le général Giap et ses bo doi. Des films comme Apocalypse now, des romans d'émigrés écrits en anglais pour un public américain comme La nuit nous a surpris de Kien Nguyen ou Entre le ciel et la terre de Ly Hayslip, lui-même adapté dans un film américain, ont façonné une mémoire dominante de cette guerre qui s'est substituée à la mémoire coloniale française, dans laquelle chaque camp, supportant des pertes humaines et symboliques immenses, se définit malgré les antagonismes comme celui de la liberté.

Si ces images contradictoires sont souvent liées à la guerre, leur présence obsessionnelle, accordant au fond bien peu de place à la perception proprement vietnamienne de l'histoire du $x^{\mathrm{e}}$ siècle, ont surtout effacé un fait assez simple. Loin de se résumer à un champ de bataille ou à un espace colonial dont l'émancipation a déclenché tous les mouvements de libération ultérieurs, en Afrique notamment, le Vietnam a été l'un des lieux les plus frappants de rencontres entre les peuples et les cultures dans l'histoire humaine, un creuset de civilisations par rapport auquel les melting pots les plus souvent invoqués comme celui des États-Unis se résument à des équations beaucoup plus simples. Il y a certainement un moment vietnamien de l'histoire globale, les transferts culturels qui ont formé le pays donnant un écho universel aux cataclysmes qui se sont abattus sur lui. Le Vietnam a pu être, pour un temps, beaucoup plus qu'un théâtre de guerre, le miroir privilégié d'un basculement du monde.

À la vérité, une science des choses vietnamiennes s'est développée en France depuis plus d'un siècle ${ }^{1}$. Elle s'enracine dans des récits de voyage ou d'expéditions qui ont depuis longtemps cédé la place à des recherches répondant à toutes les exigences de l'érudition historique, depuis les travaux historiques de Lê Thân Khôi jusqu'aux études de Philippe Langlet sur le bouddhisme vietnamien. Nombre d'auteurs de cet ouvrage font partie de la famille des vietnamologues, dont on pourrait dès maintenant reconstruire l'histoire, les terrains de recherche favoris, les habitudes de publication et les questionnements.

1. On pense au récit du docteur Hocquard, Une campagne au Tonkin, réédité par Philippe Papin (1999) et qui décrit le Vietnam des années 1880. 
Soixante ans après la bataille de Dien Bien Phu, il est désormais temps d'envisager le Vietnam sous l'angle des rencontres auxquelles il a servi de cadre, en soulignant ce que ces dernières, au-delà des traumatismes, générateurs de luttes fratricides mais aussi de formes et d'idées nouvelles, ont apporté à la définition d'une culture ou plutôt de plusieurs: car l'imaginaire européen ne serait sans doute pas le même sans la rencontre avec le Vietnam.

Cette rencontre entre les cultures a pris une forme qui a profondément marqué les hommes qui en firent l'expérience dans les champs où ils œuvrèrent. Que l'on pense à Han Mac Tu ou à Marguerite Duras, ou encore à Victor Tardieu et Nam Son qui créèrent ensemble l'École supérieure des beaux-arts de l'Indochine, que l'on considère la philosophie, l'architecture, la médecine: tous ces domaines témoignent de la fécondité qui en a découlé.

Dans son Kim Vân Kiêu, poème qui s'ouvre sur le thème de rencontres, notamment celle de Kim Trong et de Thuy Kiêu, Nguyen Du évoque ainsi l'union qu'elle fait naître entre les personnages, avant leur séparation:

Le jeune homme dit: " Aujourd'hui il vente, demain il peut pleuvoir. Les jours de printemps promettent-ils plus d'une rencontre heureuse? Si vous méconnaissez ce cœur éperdu, il en souffrira, certes, mais sans nul profit pour personne. Engageons-nous d'abord pour quelque mutuelle promesse. [...]

Elle dit: «Dans cette première rencontre, émue par votre cœur, comment pourrai-je faire taire mon cœur? Puisque votre cœur généreux me porte de l'intérêt, cette promesse, je l'accepte pour la graver à jamais sur la pierre et sur l'or. " [...]

Un serment fut prononcé, scellant leur union comme laque et colle ${ }^{2}[\ldots]$

Sinh rằng: "Rày gió, mai mưa, Ngày xuân đã dễ tình cờ mấy khi!

Dù chăng xét tấm tình si, Thiệt đày mà có ích gì đến ai ? Chút chi gắn bó một hai [...]" Rằng: " Trong buổi mới lạ lùng Nể long, có lẽ cầm lòng cho đang!

Đã lòng quân tử đa mang Một lời, vâng tạc đá vàng thủy chung. [...]"

Một lời gắn bó tất giao $[\ldots]^{3}$

2. Nguyen Du, Kim-Vân-Kiêu, traduit du vietnamien par Xuân-Phuc et Xuân-Viêt, Connaissance de I'Orient, Gallimard/Unesco, Paris, 1961, p. 51-52.

3. Nguyễn Du, Truyện Thúy Kiều, Institut de I'Asie du Sud-Est, Paris, 1986, p. 75-76. 
S'il est donné à la poésie d'exprimer la complexité de l'histoire humaine, elle peut refléter comment se compose, à la croisée des cultures française et vietnamienne, un tableau qui les associe comme la laque et la colle, l'idée et le motif - le tableau d'un paysage inachevé.

À qui ce livre s'adresse-t-il ? D'abord, à un public qui fut déjà celui du colloque $^{4}$. Ce lectorat était notamment vietnamien, ou d'origine vietnamienne. C'était celui de personnes ayant quitté leur pays natal.

Parmi ces lecteurs attendus, certains connaissent aussi bien Nguyen Du et Ho Xuân Huong que Victor Hugo ou Paul Verlaine, car ils se sont formés au contact de ces deux cultures. On peut penser aussi aux personnes qui sont venues travailler, étudier et se sont installées en France. À ceux qui ont rejoint la France pendant ou après la guerre d'Indochine et du Vietnam, et qui ont trouvé en France un pays d'accueil, à tous ceux qui se situent, pour diverses raisons, entre les deux cultures française et vietnamienne, aux Vietnamiens du Vietnam curieux de l'effet de miroir produit par les complexités de leur histoire.

À ce premier public s'adjoindra tout naturellement celui des Français, historiens de l'art, de la peinture, de la littérature, urbanistes, anthropologues, linguistes, traducteurs, étudiants, voyageurs, artistes qui se sont intéressés au Vietnam et ont noué des relations fortes avec ce pays. Ils ont tous une expérience vécue des sujets abordés, une mémoire dont il convient de ne jamais trop s'éloigner. Tant de visiteurs du Vietnam ou de lecteurs curieux de sa culture peuvent s'intéresser à lui, qu'ils en soient ou non originaires, comme à une part cachée d'eux-mêmes.

Quatre questions ont principalement orienté notre réflexion:

Peut-on exporter le concept de transfert culturel, forgé pour étudier les passages d'une culture européenne à l'autre, aux relations entre la France et le Vietnam? Et si oui, comment se sont élaborés les transferts culturels entre ces deux pays? Comment ces relations culturelles s'inscrivent-elles dans les arts, la littérature, les sciences, l'histoire des idées, la société? Comment ont-elles pu engendrer des idées et des formes nouvelles, à travers un mélange culturel?

Suivant ce fil directeur, nous avons d'abord choisi de voir pourquoi l'histoire culturelle du Vietnam est particulièrement propice à l'étude de transferts culturels, entre les Viêt et les minorités, entre les Viêt et les Chinois ou le Champa indianisé, avec la France ou les États-Unis, bref

4. Les contributions ont d'abord été présentées dans le cadre d'un colloque qui s'est tenu à l'ENS et à la BNF en juin 2014. Jean-Yves Mollier avait apporté une aide importante à la conception de cette rencontre. 
pourquoi un décloisonnement de l'histoire culturelle vietnamienne permet seul d'en apprécier la richesse et la complexité. L'ouvrage n’aborde pas la vie économique et sociale de l'Indochine puis du Vietnam, mais se concentre délibérément sur la culture savante, littéraire ou artistique. Étudier l'histoire de la pensée au Vietnam implique par exemple que, d'une part, l'on mette en parallèle la manière dont les Lumières ont pénétré l'espace intellectuel vietnamien et, d'autre part, qu'on prenne en compte le facteur religieux à travers deux des religions les plus répandues, le bouddhisme et le catholicisme.

Les rencontres entre les cultures aboutissent à la construction de nouveaux savoirs. Parfois l'émergence de ces savoirs s'observe dans les échanges entre les Viêt et des minorités comme les Hrê, et on ne doit pas oublier le rôle joué par la pluralité du monde vietnamien dans les connaissances anthropologiques élaborées en France, notamment dans le cadre de l'École française d'Extrême-Orient, fondée en 1898 à Saigon, transférée à Hanoi en 1902, avant de s'étendre à l'ensemble des pays asiatiques. Des savoirs mixtes se sont constitués au Vietnam dans tous les domaines. Comment en serait-il autrement alors que l'école vietnamienne et l'université vietnamienne se sont, durant la première moitié $d u x^{e}$ siècle, fondées sur des modèles français réinterprétés et adaptés au contexte vietnamien? Cette mixité s'observe au niveau de sciences particulières comme la médecine dite française dans son application au terrain vietnamien. L'institut Pasteur serait-il concevable sans ce contexte vietnamien auquel s'était tant attaché Yersin? Les récits journalistiques portant sur le Vietnam, y compris ceux que l'on trouvait dans la presse illustrée des années 1950, sont eux aussi une forme de constitution d'un savoir mixte.

Il convient de s'attacher plus particulièrement aux arts et à la littérature qui sont, en général, des marqueurs d'identité, mais qui dans le cas des relations franco-vietnamiennes pourraient être considérés comme des vecteurs de métissage. Les thèmes vietnamiens sont de plus en plus présents dans la littérature française ${ }^{5}$. Il y a l'architecture orientalisante qui se développe en Indochine et l'appropriation des paysages vietnamiens par des peintres français, tandis que les peintres vietnamiens adoptent des modèles français. Les romans vietnamiens utilisent, à côté de traditions remontant à la Chine, des modèles français, mais les contes vietnamiens peuvent de leur côté être transcrits pour un public

5. Sans remonter au Barrage contre le Pacifique (1950), songeons simplement pour ces dernières années, parmi bien d'autres exemples, à la biographie romancée d'Alexandre Yersin par Patrick Deville Peste et choléra (2012) ou au roman de Minh Tran Huy, Voyageur malgré lui (2014). 
francophone: les œuvres littéraires sont de plus en plus nombreuses à tisser les liens franco-vietnamiens comme si les deux histoires littéraires étaient liées par des liens structurels, des liens d'autant plus paradoxaux que la simple expression de la subjectivité paraît déterminée par les spécificités de deux langues très éloignées. L'évocation du Cambodge littéraire rappelle qu'on ne peut totalement séparer le Vietnam, dans ses rapports à la France, d'un ensemble plus vaste qui fut l'Indochine.

On ne saurait s'engager dans l'histoire culturelle de l'espace vietnamien sans se préoccuper des sources, et, en particulier, du fait que la Bibliothèque nationale de France est détentrice d'une part importante de la mémoire vietnamienne, qu'il s'agisse des premières photographies prises dans le pays, des traces de spectacles qui y furent données ou encore de documents imprimés et manuscrits qui invitent à s'interroger sur l'histoire de l'écriture au Vietnam.

Il serait présomptueux de prétendre aborder dans un même volume les multiples facettes du phénomène des imbrications et réinterprétations culturelles qui ont fait l'espace vietnamien. Enfin, s'il est vrai que les relations entre la France et le Vietnam sont le fruit d'une longue histoire depuis le $\mathrm{xvII}^{\mathrm{e}}$ siècle, elles continuent naturellement à se construire aujourd'hui; sans doute la référence à la France s'estompe-t-elle pour le Vietnam derrière un faisceau complexe de relations, celles que le Nord a entretenues avec la Russie, celle que le pays réunifié peut avoir avec les États-Unis. Ce volume se veut donc simplement une invitation à poursuivre l'exploration d'un champ dont il esquisse les contours possibles. 
PREMIÈRE PARTIE

\section{MISE EN PERSPECTIVE THÉORIQUE ET HISTORIQUE}





\section{Les transferts culturels franco-vietnamiens}

\section{Un cas de figure paradigmatique Michel Espagne}

La France et le Vietnam ont une histoire commune depuis 1624, date à laquelle le missionnaire jésuite Alexandre de Rhodes s'embarque de Macao pour le port de Hoi An au Sud de Danang et se prépare à un séjour de plusieurs années au Vietnam. Il en reviendra avec un premier dictionnaire de la langue vietnamienne, le Dictionarium Annamiticum lusitanum et latinum, publié à Rome en $1651^{1}$. Ce n'est toutefois pas la seule raison de voir dans le Vietnam un terrain particulièrement favorable à l'étude des transferts culturels. À côté de l'histoire franco-vietnamienne, il y a au Vietnam une histoire partagée avec la Chine, le Japon, les ethnies minoritaires pour ne pas parler des États-Unis jusqu'à la fin de la guerre du Vietnam. Chacune de ces interactions, de ces dynamiques constitue une strate dans un long déroulement diachronique.

Peut-être pourrait-on partir des perceptions les plus immédiates, de l'étrange impression de familiarité que donnent ces paysages urbains vietnamiens où la mairie, la gare, l'église, le théâtre, et le musée rappellent les diverses composantes des villes françaises sous la Troisième République. Il y a, de même, des figures partagées qui pourraient reposer dans une galerie historique franco-vietnamienne, où Yersin aurait sa place, à côté des soldats blancs de l'émancipation, comme ces légionnaires passés du côté du Vietminh, ou à côté du philosophe Tran Duc Thao ou du mathématicien Ngo Bao Chau.

1. Jean Le Pichon, France-Indochine. Au cœur d'une rencontre 1620-1820, Paris, éditions du Jubilé, 2005. 


\section{Retour sur une notion}

Pour parler de transferts culturels, il importe de rappeler qu'il ne s'agit pas d'aborder, d'une manière devenue assez banale, les relations entre des espaces culturels, qu'ils soient nationaux ou définis par des appartenances plus floues, ethniques, linguistiques ou religieuses; il s'agit encore moins de comparer ces espaces. L’objet, de façon beaucoup plus spécifique, est de mettre en évidence les points d'articulation qui les relient. Le terme de transfert implique certes une dynamique d'importation et d'exportation de biens culturels, qu'ils soient matériels ou correspondent seulement à des modes de pensée. Mais le point essentiel est la question de la réinterprétation liée au déplacement dans l'espace ou dans le temps. Un tableau de la Renaissance italienne exposé au Louvre n'a pas exactement le même sens ni la même valeur que ceux qu'il pouvait avoir dans son contexte florentin. Le Kant français transformé en idéologie de la Troisième République n’est plus le philosophe de Königsberg. Chénier ou Parny traduits par Pouchkine ne sont plus juste des poètes néoclassiques français.

Comprendre un transfert culturel implique que l'on abandonne l'imaginaire de l'influence, terme qui relève quasiment de la magie, pour s'attacher aux médiations historiquement constatables par lesquelles un livre, une œuvre d'art ou une école de pensée ou encore un objet matériel ont pu circuler. Ces médiations font intervenir des catégories sociologiques différenciées et complexes. Le négoce est évidemment un vecteur essentiel. L'existence de colonies de négociants allemands à Bordeaux ou à Nantes explique par exemple le voyage de Hölderlin au bord de la Garonne ou celui de Herder à Nantes. Les traducteurs sont aussi des médiateurs, et l'histoire de la traduction n'en est encore qu'à ses débuts. Comptent aussi parmi les médiateurs les professeurs de langue, là encore une catégorie sociale mal connue. On doit penser également aux soldats: la bibliothèque de l'armée de terre, à Vincennes, est riche en documents du XviII ${ }^{\mathrm{e}}$ siècle informant les officiers des caractéristiques culturelles de ce théâtre des guerres d'Ancien Régime qu'était l'Europe centrale. Enfin, comme les militaires et marins, les prêtres, missionnaires, pasteurs et rabbins voyagent. De façon générale, toutes les catégories sociales vouées aux déplacements d'un espace culturel à l'autre contribuent à la mise en place de transferts. L'histoire du judaïsme européen, passant au gré des contextes politiques d'un pays à l'autre et plus précisément de l'Espagne à la Hollande et à la Turquie, ou durant le cours du $\operatorname{xIX}^{\mathrm{e}}$ siècle de l'Est à l'Ouest de l'Europe est une histoire de transferts culturels. La dimension sociologique du phénomène oblige à s'occuper de populations parfois 
réduites. Il n’y a aucune relation de proportionnalité entre l'importance d'un transfert et celui du vecteur démographique. Le fait qu'une référence culturelle n'ait qu'une présence homéopathique n'autorise pas à la rejeter comme hasardeuse, sauf à considérer que le critère quantitatif est le seul pertinent en historiographie.

L'interprétation du bien culturel importé en modifie plus ou moins profondément le contenu et dépend du contexte d'accueil, de l'utilité que cette importation revêt dans le champ de tensions du contexte en question, de ses implications. L'émergence d'un sens nouveau ne peut en aucun cas être interprétée comme une déperdition de sens. Une des caractéristiques essentielles de la recherche sur les transferts est l'axiome selon lequel une traduction est aussi légitime que l'original, et selon lequel l'originalité se recrée. Le kantisme français n'a pas moins de pertinence que le kantisme allemand, le bouddhisme chinois n'est pas moins original que le bouddhisme indien. Même lorsque la transposition repose sur des informations partielles, la nouvelle configuration de sens ne doit pas pour autant être envisagée sous l'angle d'un déficit. Il existe des transferts dont l'utilisation dans la culture d'accueil reste latente jusqu'à ce qu'un besoin se fasse sentir de transformer la mémoire passive en mémoire active. S'interroger sur ces déplacements implique en général une confrontation avec les structurations même de la mémoire, celle-ci, dans l'organisation étatique des archives, visant souvent à reléguer dans ses marges ce qui ne conforte pas l'organisation de l'État. On tend trop souvent à réduire les transferts culturels à des relations bilatérales. En fait on a le plus souvent affaire à des configurations beaucoup plus complexes. Il y a une concurrence franco-allemande dans l'histoire culturelle de la Russie. La réception de l'histoire intellectuelle allemande en Italie est souvent passée par l'intermédiaire d'une étape française. Un système de traductions relais explique le passage de la littérature anglaise en Allemagne au xviI ${ }^{\mathrm{e}}$ siècle. Le même système prévaut encore lorsqu'il s'agit de faire circuler à partir d'une version anglaise des œuvres rédigées dans une langue peu connue.

Les transferts culturels sont le plus souvent envisagés dans la synchronie. Il est toutefois important de rappeler qu'on peut prendre en compte leur dimension diachronique. C'est même sur un transfert culturel diachronique qu'est fondée l'œuvre de l'historien de l'art Aby Warburg quand il étudie la resémantisation d'éléments antiques dans l'art de la Renaissance. Enfin les transferts ne se limitent pas à l'histoire moderne, même si le point de départ de ce type d'investigations est lié à l'histoire du xix siècle et des relations entre nations durant ce siècle. En effet la rencontre des Grecs de Phocée et des populations 
celtes sur le site de Marseille, la rencontre des Grecs et des Cariens vers Halicarnasse, celle des soldats d'Alexandre et de peuples nomades ou de peuples iraniens vers Ai Khanoun en Afghanistan ne sont pas des cas de figure moins pertinents que les importations en France de moments de l'histoire culturelle allemande. Il faut toutefois pour s'en apercevoir remettre en cause l'opposition entre centres culturels (en l'occurrence la Méditerranée gréco-romaine) et périphéries. Cette observation conduit à une réflexion sur la notion même de culture dans la recherche sur les transferts culturels. Il n'est pas question d'en rester à la représentation d'une culture nationale avec sa littérature et son historiographie implantées dans le cadre d'un État-nation. D’abord, les mœurs et les formes économiques font partie intégrante de la culture. D'autre part, toute forme de communauté linguistique, ethnique, religieuse susceptible de fournir un cadre de réinterprétation et d'appropriation concomitante d'objets ou d’idées importés peut servir de théâtre à un transfert.

\section{Transferts en contexte colonial}

La méthode d'approche historiographique en termes de transferts culturels s'applique avec une pertinence particulière aux pays qui ont subi une domination de type colonial. Il y a à cela plusieurs raisons. D'abord, la comparaison est particulièrement impropre à intégrer dans une historiographie transnationale des territoires caractérisés par une situation d'asymétrie et soumis à une violence structurelle. On ne peut pas comparer l'Angleterre et la Birmanie, la France et le Cameroun. Cela ne signifie pas que l'histoire des interactions soit sans intérêt d'un point de vue d'histoire globale, ni qu'elle se résume à l'histoire d'une domination. Il est au contraire tout à fait envisageable dans une perspective de transferts d'inverser la perspective coloniale. On peut observer, par exemple, que les habitudes de consommation des métropoles ont été profondément modifiées par l'existence de territoires colonisés (café, cacao, caoutchouc ou à un tout autre niveau uranium). Cette transformation d'ordre économique suffirait à montrer que les relations ne sont pas unilatérales. S'il n’y a pas grand sens à comparer la France aux pays africains, il est très actuel de montrer à quel point l'Afrique est présente à tous les niveaux de la vie culturelle, économique et même politique française. La même observation s'imposerait au niveau de la culture artistique. Le cubisme est par exemple incompréhensible sans ce que Carl Einstein appelait la " plastique nègre ». Dans les domaines littéraires, il 
conviendrait d'observer à l'époque contemporaine la place occupée par des littératures africaines dans la littérature française contemporaine.

D’Assia Djebbar à Yasmina Khadra, la littérature algérienne n’est évidemment pas en reste. Une histoire littéraire qui les ignorerait se ridiculiserait. Aborder les anciennes colonies en termes de transferts culturels implique de porter une attention particulière aux institutions de formation. Elles sont en principe, au moment de leur création, destinées à former des élites locales qui joueraient le rôle de représentants fidèles des forces de la métropole. Dans les faits, elles remplissent souvent ce rôle mais elles sont aussi parfois conduites à puiser dans les enseignements acquis dans les écoles ou universités françaises les outils qui permettront de penser la situation des colonies et d'envisager des mouvements de révolte. On peut dire que la puissance coloniale, en créant les prérequis de sa propre liquidation, montre qu'il n'y a pas de relation de domination unilatérale. D'une certaine manière, la puissance coloniale est même responsable des mécanismes identitaires, de la création d'entités nouvelles. Tout cela non seulement parce que la métropole dessine des frontières et répartit de la sorte des populations dans l'espace mais aussi parce qu'elle rassemble des données ethnographiques qui confortent le sentiment identitaire. L'ethnoanthropologie est toutefois ambiguë. Elle peut collecter des enseignements sur des peuples qui s'en serviront pour construire leur propre histoire. Elle sert en même temps de miroir dans une réflexion générale sur les sociétés. Mais elle peut aussi servir d'élément de domination en promouvant un fractionnement à l'infini de l'espace en ethnies, en langues dont les spécificités sont soulignées aux dépens des solutions de continuité. Les deux cents langues de tel pays africain ont pu être aussi un élément permettant d'asseoir une domination fondée sur une ethnicisation abusive.

L'histoire du Vietnam est un terrain idéal pour observer les phénomènes de transferts culturels entre un pays jadis colonisé et la France mais aussi, dans une durée bien plus longue, les transferts culturels à l'intérieur de l'espace asiatique.

\section{Le Vietnam ou la perpétuité du transfert}

L'histoire ancienne du Vietnam est déjà une histoire d'imbrications entre les cultures. Une importante exposition sur les arts de l'ancien Vietnam qui s'est tenue à Houston en septembre $2009^{2}$ révèle, par

2. Nancy Tingley (éd.), Arts of Ancient Vietnam. From River Plain to Open Sea, Houston, the Museum of Fine Arts, 2009. With essays by Andreas Reinecke, Pierre-Yves Manguin, Kerry Nguyen-Long, and Nguyen Dinh Chien. 
exemple, la combinaison au début de notre ère de motifs chinois et de motifs de la culture de Dong Son spécifique du fleuve Rouge. Même si les modèles chinois semblent progressivement l'emporter, des nuances locales montrent bien qu'il s'agit plutôt d'une adaptation que d'un emprunt. La culture de Sa Huynh au Sud est différente de celle qui prévaut dans le bassin du fleuve Rouge, mais des contacts sont fréquents et l'on trouve par exemple des tambours de type Dong Son dans des champs de fouilles relevant de la culture Sa Huyhn.

C'est pourtant avec l'apparition de la culture cham que le Vietnam devient le théâtre de métissages particulièrement spectaculaires. Le royaume du Champa, lui-même confronté aux Khmers, introduit le panthéon hindouiste, vénéré dans des sanctuaires sous forme de tours de briques qui constituent encore un des témoignages essentiels du Vietnam ancien, et introduit également la mythologie des textes sanscrits. Les inscriptions du Champa mettent en évidence une connaissance des principales œuvres littéraires indiennes. Si le cham est une langue dite austronésienne, l'usage du sanscrit est fréquent, et le Champa est une société bilingue; le Nord du Vietnam avec l'usage complémentaire du vietnamien et du chinois l'est également. Parfois liés aux Viêt contre les Khmers ou les Mongols, parfois alliés aux Chinois contre les Viêt, les Cham représentent en tous cas dans l'histoire culturelle du Vietnam une alternative à la culture chinoise, ou plutôt les deux modèles se combinent dans un syncrétisme dont on a fait la caractéristique de plusieurs dynasties viêt (les Ly et les Tran). On peut observer dans le domaine religieux que telle déesse mère cham (Po Nagar-Bhagavati) protectrice du pays a été intégrée dans le panthéon viêt sous forme de déesse bienfaisante et proche du peuple ${ }^{3}$. Le dieu Indra est honoré à Tang Long. L'oiseau mythique Garuda propre à la tradition indienne passe du Champa dans les motifs décoratifs de l'art viêt, tandis que l'art cham adopte depuis le $\mathrm{IX}^{\mathrm{e}}$ siècle les dragons que l'art viêt a hérités de la Chine ${ }^{4}$. Si un décret du XIv ${ }^{\mathrm{e}}$ siècle interdit de parler la langue cham en pays viêt et si un décret $d u x^{\mathrm{e}}$ siècle interdit aux Viêt de prendre des épouses cham, ces interdictions attestent par ellemêmes d'une tendance perçue comme dangereuse au métissage. Après les campagnes victorieuses contre le Champa, les armées viêt s'emparent des danseuses et des musiciens. Les Cham comme les Viêt ont payé tribut à la Chine, même si le poids du modèle chinois est plus particulièrement sensible chez les Viêt qui ont emprunté à leurs voisins du Nord les textes

3. Anne Valérie Schweyer, Le Vietnam ancien, Paris, Les Belles Lettres, 2005, p. 186 sq.

4. Ibid., p. 277-278.. 
du canon bouddhique en version chinoise. Si l'étude des parties séparées du Vietnam ancien est évidemment très importante pour comprendre l'histoire du Vietnam, elle l'est peut-être moins que celle des interactions qui la jalonnent.

Le port de Hoi An, d'abord intégré aux échanges économiques du Champa, assurait notamment les échanges avec la Chine. Mais la présence d'un pont couvert japonais montre qu'il s'agissait aussi d'un relais important dans le commerce avec le Japon. Les négociants de Hoi An avaient au $\mathrm{XVI}^{\mathrm{e}}$ siècle des connexions jusqu'à Kyoto. Mais au XvII ${ }^{\mathrm{e}}$ siècle le commerce de Hoi An était contrôlé par des marchands chinois. Une épave de navire thaï $d u x^{e}$ siècle retrouvée au large de Hoi An et transportant sans doute vers le golfe du Siam des porcelaines chinoises et des produits de l'artisanat viêt témoigne de la complexité des échanges économiques contrôlés par le port de Hoi An. Les mariages mixtes entre les marchands étrangers et les femmes locales qui jouaient un rôle considérable dans les activités commerciales semblent avoir été une règle dans ce foyer de rencontre entre les cultures. Lors de l'implantation des jésuites en Asie, la mission de Macao envoya des prêtres japonais à Hoi An où fut notamment élaboré avec l'aide de jésuites portugais le quoc ngu.

La culture de Oc Eo dans le delta du Mékong, présentée par PierreYves Manguin dans le catalogue de l'exposition sur l'art de l'ancien Vietnam, révèle, dans l'ensemble des strates culturelles dont la composition constitue le Vietnam, un ensemble très lié à l'Inde, puisqu'on a retrouvé des inscriptions sanskrites du v siècle. Pourtant il ne s'agissait pas d'une culture cham. Deux médaillons romains du second siècle de notre ère (époque des Antonins) retrouvés sur un site du delta relevant de la culture de Oc Eo confirment la dimension cosmopolite de cette culture. La cité de Fu Nan, dans laquelle certains voient le port le plus oriental évoqué par Ptolémée, le mythique Cattigara, entretenait à l'évidence des relations étroites avec l'Inde. On a trouvé des gemmes et des camées d'origine indienne, et la situation de la ville laisse supposer des relations avec la Malaisie. Le commerce international a été une des sources de revenus de cette ville de Fu nan citée dans les sources chinoises. Et l'on soupçonne la présence de commerçants d'Asie centrale, de Sogdiane ou de Bactriane.

L'enquête sur le cosmopolitisme des régions du Vietnam et sur les interactions entre ces diverses régions n'a pas été seulement un des objets d'étude de l'École française d'Extrême-Orient depuis sa fondation. La pluralité vietnamienne est aussi une des origines ou des champs d'application favoris de l'anthropologie structurale française. On pense avant tout au livre de Georges Condominas Nous avons mangé la forêt (1957). Il s'agit 
d'une description de la vie sociale du village des Mnong Gar de Sar Luk près de Dalat. Condominas perçoit d'emblée le rôle joué par l'observateur dans l'enquête: "Qui dit homme dit contexte social. Bien que j'aie rejeté le comportement habituel, "normal”, de mes compatriotes et que j'aie tenté de me fondre dans le milieu Mnong, les Hommes de la forêt ne pouvaient pas voir en moi autre chose qu'un membre - tout à fait particulier bien entendu - d'une catégorie sociale relevant d'une structure globale dans laquelle eux-mêmes ont été projetés par le fait colonial ${ }^{5}$. » Malgré cette précaution, Georges Condominas essaie de décrire la structure d'une société pour ainsi dire à l'état pur, sans s'arrêter trop longuement sur les imbrications ni avec la société coloniale, ni avec d'autres sociétés traditionnelles. Pourtant le regard structuraliste, qui a lui-même sa propre histoire, est un exemple de transfert culturel, associant un objet vietnamien et une construction herméneutique. Aussi bien les sociétés qui composent le Vietnam traditionnel que le regard porté sur elles peuvent être interprétés comme des phénomènes de transfert!

\section{Une architecture urbaine}

S'il est un domaine de la vie sociale où l'imbrication des références culturelles donne lieu à des signes clairement lisibles, c'est bien celui de l'architecture urbaine. L'architecture sert d'abord à affirmer une domination. Il n'est pas nécessaire de commencer par le Vietnam pour réfléchir au phénomène. Les sept grands immeubles staliniens de Moscou imposent dans le paysage urbain de la capitale russe un système de références destiné à en organiser la vie. Berlin a marqué son emprise sur le territoire centrifuge de l'électorat de Saxe. C'est aussi une forme de domination symbolique qui a fait organiser à des architectes allemands la ville d'Athènes selon des modèles marqués par le néoclassicisme allemand.

Pour manifester son emprise, inscrire sa domination dans l'espace urbain, la France choisit Hanoi, capitale non seulement du Vietnam mais, initialement, de l'ensemble colonial indochinois. On pense à la cathédrale Saint-Joseph, au palais du gouverneur, au théâtre municipal. Remarquons au demeurant que ces constructions tendent à rapprocher le paysage urbain de Hanoi du paysage urbain français: un rapprochement qui ne fut à l'ordre du jour dans aucun pays d'Afrique noire. Comme le montre Philippe Papin dans son histoire de Hanoi ${ }^{6}$, c'est avec Ernest Hébrard,

5. Georges Condominas, Nous avons mangé la forêt, Paris, Mercure de France, 2003, p. 10-11.

6. Philippe Papin, Histoire de Hanoi, Paris, Fayard, 2001. 
lauréat du grand-prix de Rome 1904, responsable des questions d'urbanisme à Hanoi de 1923 à 1933, que s’annonce un style dit indochinois, qui avec ses toits emboîtés et recourbés vise à produire des formes mixtes. Le bâtiment de l'École française d'Extrême-Orient, inauguré en 1931 et devenu le musée national, ou la direction des finances (1934) illustrent ce moment de mixité qui semble s'ouvrir avec l'Université indochinoise de 1927. À Saigon ${ }^{7}$, on observe une évolution comparable. Le musée Blanchard de la Rosse par Auguste Delaval (1929) ou le lycée Pétrus Ky d'Ernest Hébrard édifié la même année trahissent la volonté d'intégrer des éléments vernaculaires dans l'architecture comme dans la société. Le musée de Danang s'efforce, quant à lui, d'intégrer des moments de l'architecture cham. Il faut attendre l'église construite entre 1959 et 1962 à Hué par Ngô Viêt Thu pour que l'architecture religieuse soit aussi marquée par les tendances à la mixité des formes dont le moment essentiel est l'adaptation des toits à des traditions locales. Les bâtiments résultant de ces expériences architecturales ont été parfaitement intégrés à la vie $\mathrm{du}$ Vietnam contemporain.

\section{Dalat, ville-transfert}

On ne saurait limiter la question des imbrications culturelles à la présence ou non de formes ressenties par des architectes comme asiatiques. Il existe au Vietnam un lieu parfaitement improbable, un cas de transfert réellement impressionnant et suggestif: il s'agit de la ville de Dalat, conçue selon des normes et des projections européennes mais avec une reconnaissance de la nécessité, pour rendre viable le projet dans son intention même, de procéder à un exercice de transposition contrôlée et d'adaptation aux conditions de l'Asie du Sud-Est et des contraintes coloniales: la ville a été récemment décrite et analysée par Eric Jennings ${ }^{8}$, qui estime que sa création fut de l'ordre de la condition nécessaire pour l'existence de l'Indochine française. Dalat, c'est d'abord une oasis de salubrité dans un pays hanté par la malaria et où la mortalité des troupes françaises est extrêmement élevée. Cette oasis est d'ailleurs largement fantasmatique, puisque le climat de Dalat n'empêche pas la diffusion de la malaria sous prétexte que le lieu a une certaine allure de paysage

7. Voir Arnauld Le Brusq et Léonard de Selva, Vietnam à travers l'architecture coloniale, Paris, Patrimoines et médias/éditions de l'amateur, 1999.

8. Eric Jennings, La ville de l'éternel printemps. Comment Dalat a permis l'Indochine française, Paris, Payot, 2013. 
alpestre. Sur le plateau de Lang-Bian, des milliers de travailleurs issus des tribus montagnardes environnantes sont employés à réaliser un rêve, similaire au demeurant à ce qu'on rencontre dans d'autres colonies (Darjeeling par exemple pour l'Inde britannique). Dalat était destiné à produire des légumes européens et du vin et à les exporter sur l'ensemble de l'Indochine, à l'intention de toute la société coloniale. Les classes bourgeoises venaient y passer les vacances, utiliser les pistes cyclables, les cours de tennis ou les piscines d'un univers qui se voulait francilien. La chasse au tigre ou à l'éléphant représentait un élément d'étrangeté de bon aloi dans ce cadre à l'européenne.

L’environnement des ethnies moï permettait enfin de célébrer une prétendue hiérarchie des races qui donnait la possibilité au colonisateur de se ménager des alliés contre la majorité vietnamienne. La publication de lexiques dans les langues des minorités ethniques prouvait leur différence en l'ancrant dans une problématique réalité linguistique. Dalat devint une sorte de capitale des minorités ethniques des hautes-terres.

Le référent de la station alpestre dissimulait ainsi des arrière-pensées moins bucoliques mais très liées à la représentation en projection d'une présence française à long terme et d'une France adaptée au contexte. Le modèle ségrégationniste dans l'organisation des quartiers fut d'ailleurs progressivement remis en cause, du moins pour ce qui est de la bourgeoisie vietnamienne; l'installation de Bao Dai à Dalat conforta cette ambiguïté. Inspirée de la gare de Deauville, la gare de Dalat révèle la continuité d'une nostalgie fondatrice, mais Dalat est progressivement perçu et revendiqué par le colonialisme français comme un modèle d'intégration des Vietnamiens dans la culture française. Et ce nonobstant certains Vietnamiens se sont retrouvés dans Dalat, ville sur laquelle Han Mac Tu a écrit un poème.

Le lycée Yersin (le pastorien, découvreur du site, y fit bâtir un sanatorium) s'est efforcé de former une élite indigène composite pour laquelle le latin restait une discipline fondamentale. En 1944, l'hommage rendu à Jeanne d'Arc à Dalat inclut les sœurs Trung qui au premier siècle avant notre ère avaient chassé les Chinois du Vietnam et se retrouvent dans les désignations des groupes de scouts. En 1946, une conférence sur l'avenir de l'Indochine a lieu précisément à Dalat. Certes la ville a été une construction du colonialisme, un outil destiné à assurer la main mise des colons, un endroit où se sont cristallisées toutes les contradictions du colonialisme en Indochine. Mais à l'encontre du projet initial la ville est aussi devenue un des lieux d'apparition d'une société mixte, tout autre chose qu'une station d'altitude destinée à restaurer la santé des militaires. 


\section{Dans les beaux-arts}

Ce détournement des fins initiales, caractéristique de l'urbanisme et de l'architecture, s'observe à divers niveaux de la vie artistique. On sait par exemple, grâce en particulier à un livre de Nadine André-Pallois ${ }^{9}$, que l'Indochine a été une source d'inspiration importante pour les peintres français et que certains d'entre eux, comme Joseph Ingimberty (18931971), peintre du delta du fleuve Rouge, ont enseigné à l'école des beauxarts de Hanoi fondée en 1924. Leurs toiles ont été présentées à Paris et ils ont ainsi contribué à modifier la sensibilité esthétique du public parisien. Mais ils ont aussi formé une génération d'élèves vietnamiens à qui ils ont inculqué des modes de représentation de leur propre environnement naturel ou humain, réincarnés via l'adoption des traditions ou des supports locaux: la peinture sur soie ou la laque. Un peintre comme Nguyen Tien Chung (1914-1976) a été successivement élève (1936-1941) puis professeur (1955-1966) à l'école des beaux-arts de Hanoi; il représente des scènes de la vie vietnamienne en employant des codes picturaux empruntés notamment à Matisse. Le catalogue de l'actuel musée des beaux-arts de Hanoi fait apparaître une suite de tableaux où les traditions picturales françaises des années 1920, transformées et adaptées au contexte du Nord-Vietnam sont mises au service de représentations non seulement de la population et des paysages, mais aussi des luttes anticoloniales.

\section{En littérature}

Il n’y a rien d'original à rappeler que la référence au Vietnam fait partie de la littérature française, qui a cherché des mots et des métaphores pour rendre des impressions, des goûts liés à ce pays lointain. Il y a certes des colonialistes parmi les écrivains qui se sont intéressés au Vietnam, mais beaucoup manifestent aussi une grande aptitude à s'identifier à la population vietnamienne. On pense notamment aux reportages de Léon Werth rassemblés sous le titre Cochinchine (1926). On a, d'un côté, des tentatives de description d'impressions inédites:

L'atmosphère est lourde et poisse aux choses. Les fleurs des flamboyants s'étagent en touffes de grenadine et de minium. Les fleurs éclatantes d'Europe poussent en serre ou dans les jardins. Mais ici c'est au haut d'un arbre $^{10}$.

9. Nadine André-Pallois, L'Indochine: un lieu d'échanges culturels? Les peintres français et I'Indochine (fin XIXe-XXe siècle), Paris, Presses de l'École française d'Extrême-Orient, 1997.

10. Léon Werth, Cochinchine, Paris, Viviane Hamy, 1997, p. 27. 
Ces notations alternent avec des observations à fleur de peau du colonialisme en action:

Un soldat frappe un coolie-pousse qui ne veut pas « marcher ». Boulevard Bonnard, devant un magasin de soieries tenu par un Annamite et par sa femme, qui est lyonnaise. Cette femme s'approche du soldat et, très doucement lui dit: «Voyons mon ami... soyez gentil... » Le soldat se retourne et lui crie: «Si vous vous êtes fait naturaliser nha-qué, moi pas ${ }^{11} \ldots$ »

Le Vietnam dans la littérature française, c'est aussi le réalisme dans la description du petit blanc dont la vie marginale et semée de dangers n'a rien de particulièrement idyllique, un petit blanc dont les formes de fraternisation avec la population vietnamienne relèvent d'une sorte de complicité naturelle des laissés-pour-compte plutôt que d'une quelconque profession de foi emphatique. Ce sont les personnages de la Nuit indochinoise (1950-1958) de Jean Hougron. Il y a bien sûr aussi les découvreurs d'un monde exotique: Jules Boissière utilise le Tonkin comme cadre de ses Confessions d'un fumeur d'opium (1896), un genre littéraire déjà établi mais qui trouve un prolongement dans les descriptions fantasmatiques du Vietnam. Dans les Civilisés qui paraissent quelques années plus tard en 1905 et valent le prix Goncourt à Claude Farrère, on assiste encore à la description d'un monde vénéneux de fumeries d'opium et de maisons closes, mais qui est tout sauf une apologie du colonialisme, plutôt une dénonciation d'un monde cynique mêlée à une fascination postromantique pour le dérèglement des sens; les colons du Tonkin n'ont au demeurant jamais éprouvé la moindre sympathie pour Claude Farrère. Mais tout se passe comme si des thématiques littéraires françaises, héritées par exemple de Huysmans, trouvaient dans le Vietnam une sorte de champ d'application susceptible de subvertir le colonialisme même. Il est vrai qu'à l'époque même où Boissière célébrait l'opium, Albert de Pouvourville dans L'Annam sanglant (1898) exprimait sa fascination pour un Vietnam puisant sa force dans l'héritage intellectuel chinois. Des toponymes vietnamiens enrichissent la langue française en lui donnant des sonorités étranges tandis que le combat engagé n'est pas décrit dans la perspective du colonisateur mais plutôt dans celle que Pouvourville imagine être celle du colonisé, comme si la littérature française était devenue l'outil d'une condamnation des barbares conquérant dans le feu et le sang des terres sinisées.

À date plus récente, on pourrait même soupçonner une certaine littérature vietnamienne d'avoir, bien au-delà de la diffusion des noms qui

11. Ibid., p. 58-59. 
caractérise Pouvourville, retrouvé des rythmes liés au Vietnam. Cela a été dit souvent de Marguerite Duras peut-être marquée par une immersion dès l'enfance dans la langue vietnamienne et dans des rythmes qui auraient débouché sur une esthétique de la parataxe. On sent une aspiration à cette esthétique de la parataxe dans le style d'un autre prix Goncourt, Pascale Roze, dans son roman L'eau rouge:

La rivière n'en finit pas de dénouer ses méandres, les clochers disparaissent et réapparaissent. Enfin le quai des messageries. Une fanfare militaire les accueille, qui lui donne des frissons au cœur. Mais ce qui l'envahit avant même de descendre à terre, c'est l'odeur. L'odeur de Saigon, ce mélange lourd de vase, de sucre, la fleur du frangipanier, d'épices de saumure, épaissi par la chaleur, qui vous prend, vous saupoudre la chair et imprègne le souffle $\mathrm{e}^{12}$.

À l'inverse, on observe des modèles littéraires français dans la littérature vietnamienne. On pourrait parler du travail du très francophile Nguyen Van Vinh (1882-1936) qui, non content de proposer une version française du Kieu a aussi et peut-être surtout traduit les fables de La Fontaine en vietnamien - il a commencé dès les années 1900 - et largement contribué par ses traductions à la naissance d'une littérature vietnamienne moderne. On lui doit en effet des versions vietnamiennes de Balzac, Hugo, Molière, l'abbé Prévost, sans oublier Les trois mousquetaires d'Alexandre Dumas. Ces traductions choisies dans l'éventail d'une littérature de grande diffusion, sont en outre en quoc ngu comme la revue fondée par Nguyen Van Vinh et donc véritablement destinées à exercer une large influence sur la production littéraire. Même s'il n'est pas facile de déterminer quelles ont été les sources d'inspiration précises de Nhat Linh, par exemple dans son roman Solitude, il est évident qu'on se situe, avec le roman psychologique visant à promouvoir l'émancipation de la femme, dans le cadre d'une tradition française des romans psychologiques, de Flaubert à Maupassant, lus par l'auteur durant un séjour en France, avant même la rédaction de ses propres romans fondateurs. Les filiations sont plus claires pour les poèmes d'un Han Mac Tu déchiré entre l'extase et le péché, qu'il est plus facile de ranger dans la filiation d'un Baudelaire. Il ne s'agit naturellement pas d'inscrire Nhat Linh ou Han Mac Tu dans une quelconque dépendance, car la situation de la femme française n'est pas celle de la femme vietnamienne dans les années 1920 et le péché ou l'extase n'ont pas la même valeur dans le Paris du milieu du $\mathrm{XIX}^{\mathrm{e}}$ siècle et dans un contexte largement marqué par le bouddhisme, mais

12. Pascale Roze, L'eau rouge, Paris, Stock, 2006, p. 21. 
de suggérer l'importance du matériau français, de la référence à l'histoire littéraire française dans la construction d'une littérature nationale.

Si une littérature se définit en fonction de la langue utilisée, le lectorat fait aussi partie des paramètres à prendre en considération. Certains auteurs vietnamiens écrivent directement en français, en visant manifestement un lectorat mixte: celui d'une diaspora tout aussi rompue à l'usage du français qu'à celui du vietnamien, et un lectorat francophone prêt à développer une empathie. Une histoire littéraire ni française ni vietnamienne s'inscrit en filigrane. Quand Kim Thuy intitule un roman en français $R u$, en précisant que le mot veut dire en français « écoulement de larmes ou de sang " et en vietnamien " berceuse » le ton est donné par cette rescapée de l'épopée des boat people. Le vietnamien reste d'ailleurs présent comme une interrogation dans le texte français:

Dans le cas du vietnamien il est possible de classifier, de quantifier le geste d'aimer par des mots spécifiques: aimer par goût (thich), aimer sans être amoureux (thuong), aimer amoureusement (yeu), aimer avec ivresse (me), aimer aveuglément ( $m u$ quang), aimer par gratitude (tin nghia). Il est donc impossible d'aimer tout court, d'aimer sans sa tête ${ }^{13}$.

Ce type de littérature comprend beaucoup d'autobiographies qui sont des descriptions de passage d'une culture à l'autre, parfois des transferts impliquant plusieurs langues et espaces comme le récit de Thi-Hien Tran Itinéraire d'une Vietnamienne. L'étudiante insoumise. L'auteur raconte l'adaptation d'une étudiante vietnamienne à la Pologne puis à la France et s'interroge d'entrée de jeu sur son identité:

Je me sens, bien que fondamentalement apatride, nostalgiquement vietnamienne, sentimentalement polonaise, virtuellement française. En moi cohabitent en permanence trois cultures, qui se retrouvent dans les plus petites choses du quotidien ${ }^{14}$.

Le roman de Kim Doan Sur place relève encore de la transposition romanesque d'une autobiographie qui s'adresse au moins autant au Vietnam qu'à la France et thématise cette double identité:

Depuis qu'elle est en transit, sur le fil des frontières, cette frontière intérieure qu'elle a durement tracée pour séparer sa part vietnamienne de sa part française s'est comme évanouie, permettant aux deux parts de s'entremêler et de se confondre. ${ }^{15}$

13. Pascale Roze, L'eau rouge, Paris, Stock, 2006, p. 21.

14. Thi-Hien Tran, Itinéraire d'une Vietnamienne. L'étudiante insoumise, Paris, Balland, 2009, p. 12.

15. Kim Doan, Sur place, Paris, Plon, 2003, p. 304. 
Peut-être faut-il intégrer dans cette catégorie des auteurs vietnamiens pour un public français des auteurs qui sont passés par la traduction, parlent essentiellement de la société vietnamienne mais vivant en France, ayant rencontré quelques problèmes au Vietnam, bénéficiant d'une très large diffusion par le biais de la langue française, ont aussi une identité mixte comme Duong Thu Huong, dont les publications sont omniprésentes dans les librairies françaises.

\section{Dynamiques de la langue}

La littérature est un outil important dans la compréhension des transferts culturels parce qu'elle permet d'observer des dynamiques sociales, l'exil étant l'une des plus frappantes. C'est précisément sur ces dynamiques sociales qu'il convient de se concentrer pour éviter que les transferts culturels ne soient saisis que sous la forme d'inventaires statiques. La langue semble être un domaine particulièrement intéressant. Il est inutile de revenir sur le rôle d'Alexandre de Rhodes dans la création d'une forme d'écriture latine complétée de signes diacritiques pour transcrire le vietnamien. Le quoc ngu a eu d'abord une fonction essentiellement religieuse, servant à des missionnaires, donc à des Européens, même si les premières ordinations de Vietnamiens sont intervenues très tôt. C'est autour de 1900 et sous la pression des autorités coloniales que cette translittération primitive devient le mode naturel d'écriture de la langue vietnamienne. Cette adoption a des conséquences bien connues qui sont, d'une part, une déconnexion des modèles chinois et, d'autre part, un rapprochement de formes européennes et françaises. Le journal Phong hoa, fondé par Nhat Linh à la suite de son séjour en France et riche de caricatures politiques sur les colons et le petit peuple vietnamien, caricatures accompagnées de légendes humoristiques, serait une imitation orientale du Canard enchaîné ${ }^{16}$. Indépendamment de la graphie, la langue elle-même s'enrichirait rapidement de termes français, parfois des reproductions purement phonétiques pour désigner la cuiller, la fourchette ou le fromage, le biscuit, l'omelette, l'essence ou la valise, parfois une tentative de former à partir de composants vietnamiens un nouveau terme du champ politique ou social comme colonialisme, féminisme ou syndicat ${ }^{17}$. Mais il s'agit aussi parfois de transformations touchant plus

16. Nguyen van Ky, La société vietnamienne face à la modernité. Le Tonkin de la fin du xixe siècle à la Seconde Guerre mondiale, Paris, L'Harmattan, 1995, p. 191-228.

17. Ibid., p. 134-138. 
profondément aux structures de la langue, comme le passage progressif de thuong à yeu pour aimer ou même l'émergence du pronom toi pour désigner le sujet, l'individu ${ }^{18}$.

La dynamique linguistique a une incidence sur les habitudes sociales et les mœurs. Bien que les Européens ne représentent en proportion de la population pas plus d'une personne sur mille (montant toutefois jusqu'à 3,5\% à Haiphong et Hanoi à la fin des années 1930), ils ont une incidence forte sur les habitudes vestimentaires. C'est ainsi que le fameux ao dai serait une synthèse de traditions vietnamiennes et d'expérimentations de tailleurs ou dessinateurs calquant leur pratique sur les modèles de la mode occidentale, parmi lesquels un certain Nguyen Cat Tuong, (rebaptisé Le Mur) aurait ainsi créé dans les années 1930 ce que l'on tend à considérer comme la mode féminine vietnamienne. C'est d'ailleurs concernant les femmes et leur statut que l'évolution des mœurs et un certain rapprochement avec les habitudes françaises semblent avoir été les plus notables. Du blanchiment des dents au raccourcissement des cheveux, la France participe clairement, notamment à travers les retours de France, à une évolution de la société vietnamienne qui, tout en s'acheminant ainsi vers une accentuation du rôle du sujet, n'en garde pas moins un certain nombre de traditions structurantes comme le quan ho. Les transferts culturels ne font pas disparaître le contexte d'accueil mais adaptent les importations à ce contexte.

L'adaptation des importations prend parfois la forme d'un renversement. Ainsi la formation occidentale ou d'abord japonaise puis occidentale, le Japon servant de tiers dans les transferts culturels, aboutit au contraire d'un alignement. On sait que le général Giap a enseigné l'histoire, que nombre d'étudiants partis, comme le futur directeur du journal Phong Hoa, Nhat Linh, étudier les sciences en France, en revenaient avec une bonne connaissance de la culture française mais aussi des conceptions politiques très arrêtées. C'est un peu la même évolution que connut par exemple Tran Duc Thao, parti pour passer une agrégation de philosophie et revenu comme penseur d'une extension marxiste de la phénoménologie. La participation de Ho Chi Minh au congrès de Tours, son rôle dans la publication en France de l'hebdomadaire anticolonialiste Le Paria n'ont pas besoin d'être rappelés ici ${ }^{19}$, mais ils illustrent par excellence le fait que la référence à la France dans la dynamique de la société vietnamienne semble avoir souvent servi à prendre ses distances par

18. Ibid., p. 126.

19. Pierre Brocheux, Hô Chi Minh. Du révolutionnaire à l'icône, Paris, Payot, 2003. 
rapport à la métropole coloniale tout en se servant d'apports intellectuels adaptés et transformés.

$$
* * *
$$

Comme tous les espaces où l'on étudie les transferts culturels, le Vietnam donne lieu à des études différenciées, éclairant tantôt un aspect tantôt un autre. La tentative d'aborder l'histoire culturelle du Vietnam en termes de transferts culturels rencontre à la vérité plusieurs écueils. Le premier est lié à la représentation de filiations autochtones exclusives, à l'effort pour dessiner des histoires strictement nationales, compréhensibles de la part d'États-nations relativement jeunes. À l'heure où l'on doit se demander si l'histoire littéraire allemande et l'école des beauxarts de l'Indochine française peuvent vraiment être distinguées de façon systématique, l'isolement d'une histoire culturelle du Vietnam serait un véritable anachronisme. Le problème ne serait pas moindre au demeurant si l'on renonçait à intégrer à une histoire de la littérature française les points de contact de celle-ci avec la langue ou plus généralement la culture du Vietnam, que ces contacts passent par des membres de la diaspora ou par des voyageurs français. Il n'est pas possible d'étudier les relations d'un point de vue unilatéral. C'est aussi le visage de l'histoire intellectuelle française qui a été modifié grâce au Vietnam, et plus largement au territoire de l'ancienne Indochine.

La relation interculturelle franco-vietnamienne ne peut enfin pas en rester à des mécanismes d'emprunt et de resémantisation purement bilatéraux, ne serait-ce que parce que le Vietnam en tant que tel n'existe que comme le produit de rencontres multiples entre les diverses ethnies qui le composent, de rencontres avec la culture indianisée des Cham, avec la Chine, avec le colonisateur français, avec la protomodernisation japonaise ou avec l'adversaire (ou allié) américain. Cette persistance des transferts de l'Antiquité à l'époque contemporaine dans l'histoire du Vietnam fait de cet espace un terrain d'étude particulièrement fécond et invite à s'interroger sur la dimension de réaction vitale, de créativité culturelle brute qui paraît les déclencher et les caractériser dans la longue durée. 

2

\title{
Un laboratoire pour l'étude des transferts
}

\author{
Philippe Papin
}

Pour qui s'intéresse à l'épineuse question des transferts culturels, y compris dans leur dimension théorique, le Vietnam constitue un excellent terrain d'observation, et sans doute même l'un des meilleurs au monde. Quels que soient l'endroit et le moment où le regard se porte, on peut y voir à l'œuvre - grandeur nature et sur la longue durée - des phénomènes de mises en contact, d'emprunts et d'échanges qui, ailleurs, apparaissent mal ou pas assez longtemps. Au-delà du cas particulier, des circonstances, des propriétés qui n’appartiennent qu’à lui, ce pays situé à la croisée des grandes aires de civilisations orientales, et à qui plusieurs des Occidents ne sont pas inconnus, est un véritable laboratoire expérimental dans lequel peuvent être éprouvés, évalués, soumis à l'épreuve de la réalité plusieurs des modèles opératoires et des hypothèses de travail concernant la notion de transfert culturel.

\section{Maillon d'empire et croisée des chemins}

En termes de diversité, de jeux sur les échelles d'emboîtement, de poids politique, d'influence subie ou voulue, de logiques de fonctionnement, de fréquence et nature des échanges, le terrain vietnamien est d'une richesse inouïe.

À l'origine de cette richesse, il y a d'abord, sur l'axe des ordonnées, la géographie. Elle joue de deux façons. D’une part, en raison de la position du Vietnam sur la carte, immédiatement limitrophe de la Chine, celle-ci étant entendue comme un empire, donc comme une formation politique agrégative et qui ne fonctionne que parce qu'elle agrège (en écho: la 
Gaule vis-à-vis de l'empire romain). D'autre part, en raison de la configuration physique du territoire, cette longue bande de terre fermée au Nord par un arc de cercle montagneux et qui, par conséquent, n’offrait pour exutoire aux migrants poussés par la pression démographique qu'un étroit corridor longeant la mer pour se diriger vers le Sud, donc vers la terre des autres (en écho: le Pérou Inca qui a glissé le long de la cordillère des Andes vers les terres du Chili actuel). Accroché par la tête à l'Empire, le Vietnam en était dans sa partie basse une marche, une frontière à la fois de défense et d'extension, autrement dit le dernier maillon provisoire: celui qui, selon les circonstances, stoppe la chaîne ou assure sa prolongation.

Sur l'axe des abscisses, c'est évidemment l'histoire. Pour s'en tenir à l'essentiel, disons que le Vietnam - en tant que peuple ou que construction politique - a rencontré quatre protagonistes:

- Tout d'abord l'empire sinisé, à l'origine de la création des deux premiers États implantés sur son territoire: le Âu-Lạc, puis le Nam-Việt, aux $\mathrm{III}^{\mathrm{e}}$ et $\mathrm{II}^{\mathrm{e}}$ siècles avant notre ère. L'empire, colonisateur direct pendant toute la durée du premier millénaire, plusieurs fois envahisseur par la suite, fut aussi un modèle d'inspiration pour l'élite vietnamienne, singulièrement à partir $d u \mathrm{xv}^{\mathrm{e}}$ siècle quand elle mit en place une monarchie centralisée appuyée sur la classe des mandarins. Il est bien sûr l'élément étranger culturellement dominant — jusque dans sa déclinaison contemporaine, maoïste hier, libérale à orientation socialiste aujourd'hui - et forme ainsi la toile de fond de l'histoire des transferts.

- Ensuite le Champa, brillant foyer de civilisation qui s'étendait sur les deux tiers méridionaux du Vietnam actuel et qui, du viII ${ }^{\mathrm{e}} \mathrm{au} \mathrm{Xv}^{\mathrm{e}}$ siècles, a fait circuler les cultures indienne et javanaise, l'hindouisme, le sanscrit, l'islam et la tradition commerciale maritime. Peu à peu grignoté par les Vietnamiens partis à la conquête du Sud, ou pour le dire autrement: s'étant peu à peu agrégé au royaume vietnamien — notez combien la manière de dire change tout - il disparaît politiquement en 1471, définitivement à la fin du XviII ${ }^{\mathrm{e}}$ siècle.

- En toile de fond, mais moins connue que les précédents protagonistes, la mosaïque des principautés internes à l'espace vietnamien, ces formations politiques et sociales qu'on appelle, en bloc et en usant d'un vocabulaire stalino-colonial, les ethnies minoritaires - comme si leurs caractéristiques ethniques étaient seules capables de les définir. Ce monde hétérogène, atomisé, moins influent que la Chine et le Champa, n'intervient vraiment dans l'histoire du Vietnam qu'après le $\mathrm{xv}^{\mathrm{e}}$ siècle, 
et surtout durant la période contemporaine. Il est, politiquement, socialement, culturellement et démographiquement très divers selon les cas et selon les moments. Il n'y a rien de commun entre les puissants gouvernements des Thai (ils sont encore quatre millions aujourd'hui), les commanderies mường (un million), les Thổ du Nghệ-An et du ThanhHoá Hoa (soixante-dix mille) et la poussière des petits groupes ne contrôlant qu'un territoire étroit, comme les Chứt, les Miao, les Khơ-Mu, les Hrê, les Ma, etc. Cela dit, le nombre n'est pas forcément un critère. Par exemple les Poong, qui ne sont plus qu'une centaine, portent encore dans leur langue l'héritage de ce qu'était le vietnamien avant qu'il ne fût sinisé, ce qui est d'un intérêt capital. Quoi qu'il en soit, cette diversité contribue grandement à la richesse du laboratoire vietnamien car, parmi les quelque vingt-cinq ou trente groupes ethniques qui ont été actifs dans l'histoire des échanges, il y en a toujours un qui correspond aux problèmes que se pose le chercheur.

- Le monde occidental enfin, à commencer par la France coloniale, un autre empire qui surgit au moment où le Vietnam des Nguyễn s'est lui-même mué en empire régional. Ce qui cependant change tout, cette fois, c'est la distance géographique et culturelle entre les deux éléments soudain mis en contact. On tient donc là, encore, une configuration extrêmement féconde à étudier. Il en va de même d'ailleurs de la seconde grande culture européenne qui a joué un rôle au Vietnam, et qu'on oublie trop souvent alors que son empreinte est bien plus nette que l'empreinte laissée par la France: la culture communiste soviétique, internationaliste à la mode du Komintern, bureaucratique dans sa conception mais qui a touché une grande partie de la population et qui est pourvue d'une indéniable homogénéité.

On voit qu'autour du maillon vietnamien s'enroulent, pour s'y accrocher ou non, des figures de transferts qui sont multiples, variées, constantes et compliquées. Au dehors: la Chine, le Champa, la France et le bloc de l'Est; au dedans: des potentats relevant d'autres cultures et possiblement concurrents de la monarchie, des moyennes et petites puissances, des isolats très faibles. Au total, ce n'est pas moins d'une dizaine d'intervenants qui, localement, se rencontrent et s'entremêlent.

\section{L'emprunt, signe d'activité politique et sociale}

Si maintenant l'on s'interroge sur la manière dont se sont établis les contacts entre ces intervenants, il convient d'abord de rappeler cette idée 
générale, et maintenant largement admise, que les influences culturelles - comment dire autrement? - ne s'exercent jamais dans un seul sens. Plus: quand on dit que A influence B, il faut comprendre que c'est B qui est l'élément actif. Il puise lui-même dans A ce qui l'intéresse, opère une sélection et, le cas échéant, transforme ce qu'il a importé. Ce qui rend impossible de penser le contraire, du moins avant l'époque moderne, c'est qu'aucun État (A) ne possède la puissance de s'imposer durablement ailleurs (B), et encore moins de s'y imposer culturellement, d'autant que, sur ce dernier point, il n'en nourrit pas même le projet. À cet égard, c'est peu dire que nous sommes piégés par le vocabulaire - sinisation, vietnamisation, hindouisation, romanisation, et jusqu'à cette influence en forme d'écoulement unilatéral (influere) - qui suggère une politique délibérée d'acculturation du point d'arrivée par le point de départ: or tout, dans l'histoire, montre l'inverse.

Il existe au Vietnam mille illustrations de ce phénomène de captation. Mentionnons par exemple les tombeaux vietnamiens d'architecture chinoise dans la province de Quảng-Bình; la déesse Bhagavathi Uma devenue Po Nagar au Champa puis Thien Y A Na au Vietnam; les villas typiquement coloniales construites par et pour la bourgeoisie vietnamienne; l'introduction dans la langue du vocabulaire et de la phraséologie communiste... Dans tous ces cas, comme dans bien d'autres, on voit souvent poindre ce métissage qui, comme le dit Michel Espagne, aboutit par nature à une resémantisation. Les tombeaux contiennent de la céramique locale, la déesse hindoue devint une immortelle, des éléments décoratifs vietnamiens s'ajoutèrent ici et là aux maisons françaises, les termes bolcheviques s'ancrèrent dans le communautarisme paysan local. Dès lors, il est évident que la traduction est aussi importante que l'original et qu'il ne saurait plus être question d'établir, comme on l'a fait jadis, des degrés de pureté par rapport à un étalon de référence. Du reste, cet étalon de référence est lui-même une vue de l'esprit car on ne le trouverait nulle part, et surtout pas dans le foyer d'origine (A) « Rome n'est plus dans Rome ", y compris quand Rome est vietnamienne.

La captation sélective, qui promeut $B$ comme élément actif, ne signifie pas pour autant que l'ensemble de B soit concerné. Ce ne sont pas «les Vietnamiens " qui mobilisent et utilisent des référents culturels chinois, cham ou français; ni "les Cham " qui puisent au stock culturel vietnamien; ni "les Thai ", "les Thổ ", "les Khmers " ou "les Khơ-Mu " pris globalement. Ce sont, seulement, certains d'entre eux. C'est l'élite, parce qu'elle en a besoin, parce que c'est son intérêt, parce que ces emprunts, une fois assimilés, servent ses propres objectifs, lesquels sont des objectifs 
à portée locale. Les hommes et les femmes enterrés dans les tombeaux d'architecture typiquement chinoise, de même que les dignitaires religieux qui ont importé Bhagavathi et les riches colonisés qui se sont offerts des villas françaises étaient tous au sommet de la société vietnamienne de leur époque. Ce sont eux, non les individus ordinaires, qui ont tiré richesse et prestige de leur association à l'empire ou à la culture exogène dominante. L'élite, quand elle transfère, s'emploie à manifester ce transfert et à s'en targuer, et elle le fait parce qu'il a, pour elle, une utilité. Le mouvement, dont on a déjà dit qu'il allait en réalité de $\mathrm{B}$ vers $\mathrm{A}$, par exemple du Vietnam vers l'Empire chinois, et non l'inverse, n'est pas initié par le peuple, la nation, l'ethnie ou toute autre généralité creuse, mais par un groupe dirigeant précis dont les membres s'insèrent dans un dispositif politique et social concret.

\section{Usages, contextes et diversité}

Car, de fait, l'élite n'est jamais faite d'une pièce. Elle est composite. En son sein, tout le monde n'a pas des besoins identiques et le recours à l'emprunt constitue bien souvent la manière dont une fraction se distingue d'une autre. Pensons aux mandarins du Vietnam du Nord qui, parce qu'ils étaient tenus à l'écart par la cour de Hué, n'ont pas beaucoup résisté à l'alliance avec les autorités coloniales, à la grande différence de leurs homologues d'Annam. Il faut donc y regarder de près. Et y regarder à chaque fois, dans chacune des configurations, puisque les affaires d'intérêt sont toujours des affaires de contexte. L'élite du Champa n'était pas de même nature que celle du Vietnam ou des gouvernements thaï, ni n'avait les mêmes aspirations.

En outre, à l'intérieur d'un même pays ou d'une même population, le groupe dirigeant a varié au fil du temps, sa composition son volume et ses mécanismes de domination se sont modifiés, de sorte que ce qui était vrai à une époque ne l'est plus à l'époque suivante. Même quand les choses se ressemblent, il faut penser a priori qu'elles diffèrent, et par exemple ne pas croire, sur la foi des apparences et du mot confucianisme abusivement employé, que l'élite du Vietnam sinisé de la seconde moitié $\mathrm{du} \mathrm{Xv}^{\mathrm{e}}$ siècle fonctionnait de la même façon que celle du Vietnam sinisé au xix ${ }^{e}$ siècle. Un gouffre les sépare, elles n'ont pas puisé dans l'Empire les mêmes références, leurs emprunts politiques et culturels sont différents parce que leurs besoins l'étaient aussi. Qu'elles aient l'une et l'autre joué à plein du transfert culturel - leur seul point commun - ne préjuge ni du contenu de celui-ci, ni de ses raisons exactes, ni des acteurs qui l'ont 
mis en œuvre: l'histoire, en tant qu'étude d'un faisceau de circonstances, demeure la principale clé de lecture de la réalité.

La conséquence, c'est que les phénomènes de transfert, s'ils sont bel et bien internes à une fraction de l'élite des sociétés réceptrices, n'aboutissent pas du tout à une uniformisation. Bien au contraire: c'est justement parce que ces fractions d'élite sont à la fois diverses selon les lieux et changeantes selon les époques qu'elles entretiennent chacune des rapports spécifiques avec le patrimoine culturel extérieur. Elles n’empruntent pas nécessairement les mêmes éléments, elles ne les utilisent pas toujours de la même façon, et dès lors il n'y a pas standardisation du point d'arrivée mais, à l'inverse, création de constructions à chaque fois originales. Pour le dire autrement: un même A produit plusieurs B parce que ce sont des $\mathrm{B}$ différents qui s'en sont inspirés. Le point A restant à peu près fixe, on ne peut nier qu'il existe une tonalité commune (l'Asie sinisée, l'Europe romaine), mais, quand on observe les choses dans le détail, on ne peut manquer d'être frappé par les dissemblances. Pour reprendre notre exemple, le modèle chinois adopté par les souverains de la dynastie des Lê au xve siècle n'a rien à voir avec celui qu'appliqueront les Nguyễn trois siècles plus tard; il s'agit assurément d'emprunts dans les deux cas, et d'emprunts puisés à la même source géographique, et cependant ce ne sont pas les mêmes références, les mêmes livres canoniques, les mêmes traditions, le même contexte, ni la même élite, si bien que le rapprochement des deux épisodes ne relève que d'un jeu sur l'apparence des mots (le « modèle confucéen »).

Faisons le point: le transfert de A vers B signifie que B est l'élément actif, il est commandé par une logique d'utilité sociale, il est le produit de son temps et présente une extrême diversité de contenus. Reste un problème très épineux qu'il faut maintenant aborder de front: l'idée que ce qui est transféré est nécessairement modifié, traduit, adapté, recomposé.

\section{L'adaptation est-elle systématique?}

On voit bien l'intérêt théorique, et même un peu politique, de la proposition. Une adaptation systématique renforcerait le rôle de B en lui ôtant d'emblée toute fonction subalterne, toute suspicion de suivisme et d'imitation servile. Elle annulerait d'un coup ce que la reconnaissance d'un emprunt fait à l'extérieur pourrait avoir d'humiliant pour le génie local. En attribuant à celui-ci une créativité systématique, une capacité permanente à transformer les choses, elle balaie à l'avance l'accusation de copie et, dans la foulée, ce qui lui paraît être un corollaire: l'idée du 
sous-produit. D'autre part, la proposition est d'autant plus séduisante qu'elle rencontre mille illustrations dans les faits. Partout, toujours, on pourra trouver des cas précis d'adaptations locales prouvant que ce qui existe ici n'est plus exactement ce qui a été pris là-bas. Cependant, la question de fond à laquelle il faut répondre n'en demeure pas moins celle-ci: la transformation est-elle systématique?

Rien n'est moins sûr. Quand on regarde la réalité froidement, on peut même penser que la transformation est un cas de figure minoritaire, un arbre qui cache la forêt, et que l'essentiel des emprunts consiste en de pures duplications. L'architecture funéraire en fournit un exemple, qui prête à développements en comparaison, par exemple avec ce qui s'est produit dans le bassin méditerranéen pendant l'Antiquité. Mais, pour en rester au Vietnam et sans multiplier les exemples, songeons à des choses aussi simples que la toponymie, l'organisation administrative, les principes de la légitimité politique (l'empereur fils du ciel et détenteur d'un mandat), l'architecture religieuse et civile, la réglementation. En toute honnêteté, que dire du code civil et pénal de Gia-Long, en 1812, dont les 398 articles sont la traduction mot à mot du code chinois du XVII ${ }^{\mathrm{e}}$ siècle? Que dire des examens mandarinaux entièrement tournés vers la connaissance des classiques chinois? De l'usage de l'écriture littéraire noble, le chinois, qui a toujours fait obstacle à l'écriture démotique? De l'imagerie populaire du Nouvel An? Du temple de la littérature qui est la reproduction, en plus petit, du temple de Qufu dans la province du Shandong? De tout cela, on ne peut dire qu'une chose: ce sont des emprunts directs, ou, si l'on préfère, des types d'emprunts au sein desquels la duplication l'emporte largement, sinon totalement, sur l'adaptation.

Bien sûr, on trouvera toujours un petit élément de couleur locale enfin non, pas toujours: parfois. Telle forme de tuile, telle couleur sur les images, telle posture d'une statue, par exemple, mais enfin ce qui saute aux yeux et ce qui caractérise le toit, l'image et la statue, c'est qu'elles sont ultra-majoritairement d'inspiration chinoise. Il arrive un moment où il faut compter, mesurer, qualifier, peser ce qui va dans un panier de la balance et ce qui va dans l'autre, et non transformer en caractéristiques saillantes de légères variations qui, quand elles existent, ne sont d'ailleurs pas très différentes de ce que seraient des variations régionales en Chine même. L'exception n'est pas plus la règle que l'arbre n'est la forêt.

Plutôt que de prendre des exemples faciles, comme l'architecture bouddhiste ou la prose savante, essayons même de nous pencher sur le cas du roman Kim Vân Kiều qui est souvent cité par les tenants de l'adaptation systématique. Ce roman d'origine chinoise a été réinterprété au 
Vietnam, comme le Cid en France, et dans une version qui est devenue meilleure que l'original. Certes, mais il n'empêche que, comme dans son modèle, l'action du roman se passe en Chine, sa trame est identique, les vicissitudes que traversent les personnages sont les mêmes, les noms des personnages sont chinois, les lieux et les références culturelles aussi. Il en va de même des romans Lục Vân Tiên, Nhị Độ Mai, Lý Công truyện, etc. Ce qui est original dans le Kim Vân Kiều, c'est la langue, c'est le vietnamien qui passe par l'écriture démotique, c'est la belle et puissante métrique en vers 6-8, c'est l'ensemble du travail stylistique - et c'est évidemment l'essentiel, personne ne prétend le contraire. Mais, dans le cadre d'une œuvre littéraire, cette originalité est une originalité par définition. Si je traduis ou translittère, je crée; si je change de mots, je change de sens. Il ne peut en être autrement (à la différence des exemples faciles que sont la littérature en chinois et l'architecture pour lesquelles il était concevable, et la plupart du temps effectivement réalisé, de dupliquer à l'identique). Le Kim Vân Kiều est un merveilleux roman - grâce à la langue, au style, aux inflexions de sens - mais, pour la question précise qui nous intéresse aujourd'hui, le fait saillant est qu'il ne contient pas l'ombre d'une vietnamisation du système référentiel ni des structures narratives. À cela, beaucoup de raisons, la principale étant que ce n'était pas nécessaire. C'eût été même déclasser l'histoire et son auteur que d'évoquer l'environnement local. Écrire en démotique était déjà une avancée énorme car, jusqu’au cœur du $\mathrm{xIX}^{\mathrm{e}}$ siècle, un lettré vietnamien aurait été horrifié de devoir écrire en vietnamien, comme son homologue français avant du Bellay de ne pas utiliser le latin. Ainsi, dans cette affaire de fidélité consentie à l'original, c'est l'usage, l'intérêt, la stratégie, l'utilité (ici: ennoblir, faire voyager, faire rêver; ailleurs: se distinguer du vulgaire) qui prime et qui explique le geste.

\section{La reprise et la réplique}

Il n'y a rien de surprenant dans ce processus d'emprunt sans transformation. Il est fréquent. Après tout, on voit mal ce qu'il y a de gaulois dans les demeures des riches Gallo-Romains du type de celle de Vaisonla-Romaine, ou bien, hormis un nom gravé, dans un monument comme l'arc de Germanicus à Saintes. Et dirait-on que le droit français est du droit romain métissé de droit druidique? Évidemment non, et ça ne lui retire rien, sauf aux yeux des chauvins; je dirais même que cela lui ajoute et, pour reprendre la belle analogie dont usait Julien Gracq à propos du roman, que le transfert culturel est, en l'espèce, un épiphyte. Sans aller 
chercher aussi loin, nous avons tous les jours sous les yeux des illustrations de duplication à l'identique, et on se donnerait bien de la peine, sinon du ridicule, à identifier ce qui est espagnol dans le traîneau du père Noël tiré par des rennes, allemand dans le port du jean, italien dans la fête d'Halloween, ou français dans les parades de majorettes d'avant-hier, la pyramide du Louvre d'hier et les élections primaires d'aujourd'hui... En outre, et j'insiste sur ce point, admettre la pure duplication ne remet pas en cause l'idée que B est l'élément actif. Il le reste parce que, comme dans l'adaptation, c'est lui seul qui est à l'origine du mouvement et qui est son moteur. En revanche, ce à quoi la duplication nous fait renoncer, c'est à l'idée que la transformation est inscrite dans la logique de l'emprunt. L'élément B est actif, oui, mais pas forcément à métisser ce qu'il transfère.

À ce stade, il faut être clair et dissiper toute ambiguïté. Il n’y a aucun jugement de valeur dans cette manière de voir les transferts culturels, aucune connotation d'infériorité posée sur celui qui importe à l'identique, aucun retour en arrière méthodologique. Remarquons, du reste, que la position inverse comporte des dangers d'interprétation malveillante qui ne sont pas moindres: dire que l'adaptation est constante, qu'on ne cesse de réinterpréter, d'ingérer, de faire sien l'étranger, pourrait laisser croire à la surestimation d'une culture exogène jugée si attractive qu'elle ne peut que séduire et qu'être appréciée. C’est ainsi: d'un côté comme de l'autre, la question des transferts culturels est remplie de pièges. Et ces pièges sont largement causés par le fait que personne ne peut échapper, dans l'énonciation, à des termes tels que "Vietnamiens ", "Chinois " ou "Gaulois ", et à leurs noms de pays, qui sont d'usage commode mais suggèrent insidieusement l'existence d'un état culturel chimiquement pur, en tout cas antérieur au contact qu'on étudie, et sur la base duquel on juge du degré d'étrangeté de l'import. C'est parce qu'on qualifie de chinois les tombeaux où étaient enterrés les dignitaires vietnamiens du premier millénaire que ces tombeaux semblent exogènes, donc empruntés; mais tout changerait dans notre perception si on les estimait communs à toute l'Asie orientale: ils seraient "asiatiques", comme les anciens Vietnamiens, et l'affaire s'arrêterait là. Aux époques anciennes au moins, ce qui nous paraît aujourd'hui extérieur, emprunté, n’était probablement pas si étranger aux populations locales que nous le croyons, sans compter qu'à leurs yeux il n’y avait sans doute pas de différence de nature entre un emprunt fait " à l'étranger " et un autre fait à la ville d'à côté ou à la capitale... Aussi bien, contre une tendance de l'esprit quasiment naturelle, il faut se résoudre à penser que les blocs culturels idéaux-types n'existent pas. Les Vietnamiens, les Gaulois, tous les autres sont déjà, au moment 
où l'on prononce le mot, y compris en l'assortissant d'une qualification liée au statut social (" l'élite vietnamienne »), le résultat d'une infinité de transferts à quantité, qualité et modulations variables.

\section{Vertu de la duplication}

Et quand bien même? Et si, réellement, le caractère exogène était patent? C'est un cas de figure fréquent dans le passé, ainsi que je l'ai dit plus haut, et qui, tout compte fait, est encore plus visible à l'époque contemporaine. Le Vietnam colonial, socialiste et actuel en offre des brassées d'illustrations, et indéniables parce qu'elles concernent non les voisins immédiats mais des aires culturelles éloignées, distantes, avec lesquelles il n’avait eu aucun contact par le passé. De fait, il se trouve qu'il a existé un certain Vietnam francisé, puis russifié et maintenant américanisé — on dirait bien sûr la même chose, en changeant les références, de la France, de l'Angleterre ou de l'Italie, mais il est vrai que le phénomène de reproduction culturelle apparaît plus nettement dans le laboratoire vietnamien. Or, et c'est là où je voulais en venir, de tous les types de transferts culturels, c'est justement la duplication à l'identique qui est la plus riche de sens.

Qu'on mêle les choses étrangères aux siennes est attendu. Mais qu'on les conserve telles quelles, qu'on s'évertue à montrer qu'elles sont exogènes, qu'on revendique leur caractère étranger : voilà ce qui est significatif. Le geste dénote une stratégie sociale et politique, une volonté de se distinguer du vulgaire, du local, de l'ancien, en arborant comme un trophée sa familiarité avec une référence jugée légitimante. Si les mandarins étaient sélectionnés sur leur connaissance des classiques chinois, parfaitement inutiles dans leur travail quotidien, c'est parce qu'ils leur conféraient du prestige auprès de leurs administrés: n'importe qui savait comment réparer une digue mais il fallait être très cultivé, très impressionnant, un peu étrange par son savoir, pour jouir de l'autorité permettant de lever la corvée villageoise qui allait s'en charger. Quand le satrape perse Mausole fit construire son tombeau à Halicarnasse, avec un propylée, une krépis, une frise sur laquelle figure Héraklès, le tout étant surmonté d'un quadrige, il voulait affirmer dans la pierre son appartenance à l'illustre civilisation grecque, et non pas achéménide. Auguste aussi, à Rome, s'est donné pour dernière demeure un monument de type grec. Et en 1070, soixante ans après l'indépendance, la fondation par le souverain vietnamien Lý Thánh Tông du temple de la Littérature, voué à Confucius, réplique du modèle chinois, signifie qu'il dote le Vietnam du type de bâtiment que se doit de posséder toute nation reconnue autonome 
au sein de l'Empire céleste. Ce faisant, il définit symboliquement mais parfaitement, et avec beaucoup d'audace à l'époque, la position politique de son royaume: associé et non sujet, dans l'empire et non en dessous. Alors, une copie? Oui, et justement parce que seule une copie, en tant que duplication parfaite d'un symbole, pouvait manifester l'aspiration à l'égalité.

Les duplications culturelles voulues par Mausole, Auguste et Lý Thánh Tông ont été des actes conscients de stratégie politique, de même que l'arc de Germanicus érigé par un descendant de Gaulois et la villa coloniale d'un riche Vietnamien furent des actes conscients de stratégie sociale. La culture revendiquée, en tant qu'elle qualifie ostensiblement son possesseur, a partie liée avec la volonté de se distinguer des uns et de se rapprocher des autres. Or on ne se distingue jamais mieux qu'en se donnant pour étranger, donc en dupliquant l'exogène. Il n'y a pas d'adaptation, pas d'acclimatement, pas de transformation, mais maintien délibéré des traits du modèle jugés caractéristiques, voire leur accentuation jusqu'à la caricature. Quitte à faire de l'étranger, autant le faire franchement et prendre non de l'avant-garde, non la dernière mode, mais le parfait stéréotype qui sera compris par tout le monde au sein de son propre environnement politique et social. C'est, entre parenthèses, toute l'histoire du confucianisme vietnamien. En attendant, malgré les apparences, malgré aussi la petite gêne qu'on éprouve à renoncer à la belle idée du métissage systématique, cette sorte d'emprunt appartient pleinement à la famille des transferts culturels. Elle en est même, sous ses allures un peu frustes ou vieux jeu, l'un des membres les plus sophistiqués. Sans prétendre que la duplication soit la modalité dominante du transfert (mais qui sait?), il importe de reconnaître qu'elle existe et que la compréhension de son mécanisme - à commencer par son origine: non pas $\mathrm{A}$ mais $\mathrm{B}$, résolument - ouvre les portes de la signification sociologique des contacts et de la profondeur historique.

\section{Rupture, déconnexion, placage de références nouvelles}

C'est sur ce dernier point qu'il faut tâcher de boucler la boucle. Il existe en effet une relation de fond entre l'idée que les transferts sont toujours des métissages et l'idée que l'histoire est faite de continuités. Dans l'autre camp, on trouve la relation opposée: entre l’idée que le transfert n’implique pas nécessairement la transformation et l'idée qu'il existe des ruptures franches en histoire. Ce sont deux positions, qui chacune ont 
leur logique. Je me rallie plutôt à la seconde. Je ne crois pas que l'histoire soit toujours faite de transitions, de porosités lentes, d'enchaînements, de passages insensibles d'un état à l'autre, le précédent se dupliquant en partie dans le suivant, le suivant gardant en lui la marque du précédent. Je ne crois pas que le mécanisme majeur soit, en histoire, celui de la pérennité, de la perpétuation et de la persistance. Et donc je ne pense pas qu'existent les traditions culturelles - en tant que corpus disponible à un moment donné, oui; mais pas comme chaînage d'une époque à l'autre. Dès lors, il est pour moi concevable qu'un temps historique soit déconnecté de l'épisode qui l'a précédé, qu'il surgisse en bousculant tout, qu'il ne se raccroche à rien de local, rien d'ancien, rien de déjà-vu, et qu'il soit en définitive parfaitement exogène.

Prenons un exemple concret. Tout le monde sait qu'on ne comprend l'ancien système politique vietnamien qu'en référence au modèle chinois, au point qu'il faut souvent aller chercher dans le second ce qui permet d'éclairer les zones d'ombre du premier, moins documenté. Je ne dis pas qu'en cette matière tout venait de la Chine impériale: je dis que l'essentiel en provenait. Et la période contemporaine? L'après 1954? Il est frappant de voir les tentatives désespérées qui sont faites, par les uns et les autres, toutes options politiques confondues - à moins qu'il ne s'agisse des mêmes options, mais travesties, justement... - pour recoller cette histoire socialiste à l'histoire ancienne, alors qu'elles n'ont rien à voir. Ils cherchent de la continuité et, péniblement, ils en trouvent. Ils se penchent sur la moindre petite chose qui rappelle l'ancien (car il en existe toujours, fatalement) mais négligent la rupture coloniale, laquelle a définitivement fait perdre au Vietnam son écriture, aussi bien que la rupture socialiste. Ils vont parler de la poursuite du culte des ancêtres quand ils ont sous les yeux un pays radicalement transformé depuis trois ou quatre générations, une société plusieurs fois bouleversée, détruite, recomposée, un langage nouveau, des pratiques sociales et religieuses nouvelles, et surtout d'énormes raccords avec la civilisation communiste qui est si bien connue par ailleurs, par exemple l'organisation en cellules du Parti, en comités populaires, en usines étatiques du genre " unité de production n²4D32 ", en syndicats contrôlés, en architecture civile stalinienne, en journalisme militant, en culte de la personnalité, en idéologie importée. Et ils tiendront le même discours aveugle et passéiste face aux changements qui poussent maintenant le Vietnam du côté de l'économie libérale et de l'intégration régionale au Sud-Est asiatique. Place Ba-Đình, ils n’ont pas voulu voir que l'ancienne Assemblée nationale était d'inspiration russe: ils ne verront pas que la nouvelle ressemble à celle de Séoul. 
En postulant l'existence d'un socle, d'un « ce-par-rapport-à-quoi » les choses changent ou ne changent pas, se démarquent ou ne se démarquent pas, et en concluant à la stabilité, on rejoint finalement l'attitude qui consiste à voir de l'hybridation partout, sauf que cette hybridation s'exerce cette fois dans la durée: les époques sont censées se mêler comme les cultures se mêlent, l'histoire censée tisser des liens dans le temps comme les échanges tissent des liens dans l'espace. En termes de passages, de transferts d'un pays à l'autre ou d'une période à l'autre, cette vision nie ou minimise les ruptures, les brusqueries, les étrangetés, l'irruption possible du radicalement autre. Or, pour ma part, je maintiens qu'il faut très bien connaître la Chine pour comprendre le Vietnam d'hier et qu'il est nécessaire, pour comprendre celui d'aujourd'hui, d'être informé sur l'Union soviétique, le monde communiste et sa grande orchestration politique, administrative, sociale et culturelle. Ce sont certes des références extérieures - ce qui ne m'effraie pas, d'autant que jamais je n'ai prétendu qu'elles sont exclusives -, mais ce sont aussi, et c'est un gage de leur validité, des références synchrones.

$$
* * *
$$

Le Vietnam est un magnifique laboratoire pour étudier la question des transferts. Il est si riche qu'il suscite même l'envie, à laquelle je n'ai pas résisté, de tenter des expériences un peu extrêmes et gentiment provocatrices. Il faut dire que le thème des transferts culturels est deux fois passionnant: il est d'un très grand intérêt et, par son enjeu, déclencheur d'enthousiasme et d'ardeur. Il me semble que cela tient à deux raisons principales. La première, c'est qu'il place d'emblée les acteurs au centre de leur histoire, faisant devenir internes des phénomènes qu'on a trop longtemps vus comme externes, ce qui débouche sur des interrogations liées aux causes et aux buts des articulations, des contacts, des échanges, et non pas simplement à leurs modalités. Derrière, c'est donc nécessairement toute une vision sociale qui se profile et la manière dont on envisage les transferts en dit long sur la manière dont on conçoit le fonctionnement d'une société. La seconde raison est que l'échange culturel véhicule beaucoup plus que de la culture: il transporte du politique, du rapport de force, de la stratégie, de la domination. Il clive, comme on dit. Et c'est justement parce qu'il clive, parce qu'il est conçu pour cela, qu'il est de plain-pied dans le domaine de l'histoire, du mouvant, du changeant, de la constante recomposition des forces à l'œuvre dans une société donnée. L'une et l'autre de ces raisons s'unissent en ce constat: l'étude des 
transferts culturels est l'antidote à la maladie infantile de l'histoire des contacts qui consiste à associer une culture à un peuple et ce peuple à un territoire, donc à emboîter trois généralités l'une sur l'autre pour finalement créer des blocs qui n'existent pas.

J'ai essayé de dire que ces transferts culturels pouvaient fort bien prendre la forme de la reproduction, et que cette reproduction avait un sens. Je voudrais ajouter pour finir qu'il faudrait en réalité, si l'on veut faire avancer le débat, s'employer à mesurer, compter, évaluer en plusieurs configurations - disons: plusieurs cas et plusieurs époques - ce qu'ils contiennent d'adaptation et ce qu'ils contiennent de réplique. Il y a peut-être là, en ce critère de la plus ou moins grande proximité avec l'original, de quoi établir les gradients d'une typologie qui, nous informant sur la densité du transfert, nous renseigne aussi sur la portée de ce qui est transféré. 


\section{DEUXIÈME PARTIE}

\section{HISTOIRE DE LA PENSÉE}





\section{3 \\ Vents d'Est, vents d'Ouest \\ L'introduction de l'esprit des Lumières au Vietnam au début du $x x^{e}$ siècle. Phuong Ngoc Nguyen}

À partir du milieu du XIx ${ }^{\mathrm{e}}$ siècle, le royaume Dai Nam des Nguyễn passe progressivement sous l'autorité française: après l'attaque du port de Tourane (actuellement Đà Nẵng) en 1858, Saigon est prise en 1859, le Sud du pays devient une colonie française sous l'appellation de Cochinchine à partir de 1862 et le reste du pays, divisé en Tonkin et Annam, entre définitivement en 1884 dans l'Empire sous le régime du protectorat. La langue française étant la langue de l'administration coloniale, on forme des interprètes et autres auxiliaires indispensables à la marche des services. Il est logique de penser que les idées des Lumières ont dû s'introduire au Vietnam directement dans les fourgons du français ${ }^{1}$. La réalité est plus complexe.

$\mathrm{Au}$ début $\mathrm{du} \mathrm{xx}^{\mathrm{e}}$ siècle, tout en s'intégrant dans l'empire colonial français, le Vietnam continue à partager avec d'autres pays asiatiques, par l'intermédiaire des caractères chinois, les fondamentaux d'un espace sinisé millénaire. Grâce aux livres et à des journaux en chinois, appelés " nouveaux écrits " (Tân Thu') par opposition aux classiques confucéens, des lettrés vietnamiens apprennent des nouvelles du monde et font connaissance avec des penseurs occidentaux. La réussite japonaise et les activités des réformateurs chinois leur donnent matière à réflexion et les encouragent à passer à l'action. Malgré la dure répression coloniale

1. Ce sera effectivement le cas de la génération de 1925, dont un des plus brillants représentants est le général Giap, admirateur de la Révolution française et vainqueur des armées françaises à Dien Bien Phu en 1954. Sur les générations intellectuelles, voir Trinh Van Thao, Vietnam du confucianisme au communisme, Paris, L'Harmattan, 1990; réédition corrigée et complétée en 2008. 
de 1908, leur remise en cause généralisée de l'ordre confucéen et leurs diverses actions (création d'écoles et d'activités économiques) contribuent à changer en profondeur et durablement la société vietnamienne.

Si ces deux thèmes - introduction de la culture occidentale et influence des " nouveaux écrits " asiatiques - ont suscité chacun une littérature abondante, ils sont trop souvent considérés à part l'un de l'autre. Or, pour bien comprendre ce processus d'introduction et de diffusion des idées des Lumières dans la société coloniale, il importe de les replacer dans l'identité de cette même conjoncture historique qui fut la leur et de saisir comment ils s'y trouvèrent en rapport, en synergie.

Il s'agirait de retracer les trajets et les modalités du passage de ces idées des Lumières, dont celles du contrat social, de la liberté et des droits de l'homme: lecture de traductions ou de textes en français, puis diffusion plus large par des moyens de communication disponibles. Il s'agirait également de mesurer l'impact de ces idées sur les générations successives. Comme programme, cela impliquerait un travail de longue haleine, nécessairement collectif.

Contentons-nous ici de situer quelques repères principaux et de décrire quelques modalités concrètes de ce processus de transfert.

\section{Début $\mathrm{du} \mathrm{xx}^{\mathrm{e}}$ siècle: crise et ouverture}

Comme le souligne Michel Espagne dans son introduction au colloque Transferts culturels: France-Vietnam, Europe-Asie, la notion du transfert implique un mouvement d'importation et d'exportation, et non pas donc un mouvement à sens unique: s'effectue un phénomène de réinterprétation et d'appropriation, une création de sens dans un nouveau contexte social. Et comme le précise Philippe Papin, dans un transfert du A vers B, il y a une « captation sélective " de B non pas par l'ensemble de la population, mais seulement par une partie de cette population qui réalise ce transfert en fonction de ses propres intérêts à un moment donné: nous conviendrons avec lui que cela vaut éminemment pour le Vietnam. Ce qui nous invite à nous demander très concrètement: qui avait lu quoi, où, quand et comment? Qu'est-ce qui fut retenu puis diffusé au sein d'un public plus large?

Rappelons tout d'abord qu'à partir du milieu du xix ${ }^{\mathrm{e}}$ siècle, le Vietnam comme l'ensemble des pays asiatiques se trouve confronté à un défi inédit: l'expansion des puissances occidentales. On connaît la réponse du Japon, qui s'emploie à partir de 1868 à contrer cette expansion par une politique d'ouverture, ouvrant l'ère Meiji, alors que la Chine, incapable de réagir efficacement, subit de plein fouet les attaques successives 
qui aboutissent au dépècement de son empire. Parmi les petits pays, la Thaïlande se singularise en prenant le chemin du réformisme dès les années 1850, notamment sous le règne de Rama V (1868-1910)².

$\mathrm{Au}$ Vietnam, les rois Nguyễn, au pouvoir depuis 1802, se montrèrent, malgré une assez bonne perception de la situation régionale ${ }^{3}$, incapables de sauvegarder la souveraineté du royaume ${ }^{4}$. À partir du milieu du $\mathrm{xIX}^{\mathrm{e}}$ siècle, de la Cour aux villages, le pays se retrouva divisé de facto en deux camps, celui des tenants de la lutte armée (chủ chiến) et celui des partisans la négociation (chủ hòa). Le mouvement Cần vương (" aide au Roi »), déclenché par l'entrée du roi Hàm Nghi en résistance à l’été 1885, se poursuivit, malgré la capture du jeune roi en 1888, une dizaine d'années. Cependant, dès septembre 1885, les Français intronisèrent Đồng Khánh, qui fut privé de toute liberté d’action.

$\mathrm{Au}$ tout début $\mathrm{du} \mathrm{xx}^{\mathrm{e}}$ siècle, les Vietnamiens font face à la situation suivante: impasse de la résistance armée traditionnelle, capitulation de la royauté, mandarinat au service du pouvoir étranger, installation a priori durable de la colonisation. Cette conjoncture plonge les lettrés vietnamiens dans une véritable crise idéologique. Le modèle de la fidélité envers le souverain (trung) est devenu obsolète, repenser le Vietnam est à présent une nécessité vitale. Découvrant la réflexion menée par des réformistes chinois et prenant acte de la réussite nipponne couronnée par la victoire sur la Russie (1905), des lettrés appellent à l'apprentissage de ce qui fait la supériorité de l'Occident. Mais si l'objectif fait consensus, les moyens diffèrent: ceux qui misent sur la lutte armée, avec pour figure emblématique le lettré Phan Bội Châu (1867-1940) s’opposent à ceux qui placent leurs espoirs dans une réforme de la société ${ }^{5}$. Ce dernier choix, tout à fait inédit, est clairement assumé, comme l'exprime le lettré Phan Chu Trinh (18721926) dans sa lettre ouverte au gouverneur général d’Indochine, rédigée

2. Jean Baffie, «Sous le règne de Rama V (1868-1910), l'adaptation du Siam à la modernité occidentale », in Gilles de Gantès et Phuong Ngoc Nguyen (dir.), Vietnam, le moment moderniste, Aix-en-Provence, PUP, 2009, p. 27-41.

3. Chen Ching-ho, « Les « missions officielles dans les Ha châu » ou "Contrées méridionales » de la première période des Nguyên », BEFEO, 1994, p. 101-124. Phan Huy Chú, Hải trình chí lược ("Récit sommaire d'un voyage en mer »), Cahier d'Archipel, 1994. Traduction et présentation par Phan Huy Lê, Claudine Salmon et Tạ Trọng Hiệp.

4. Voir Nguyen The Anh, Monarchie et fait colonial au Vietnam (1875-1925). Le crépuscule d'un ordre traditionnel, Paris, L'Harmattan, 1992; ainsi que l'ouvrage collectif Le contact colonial franco-vietnamien (Le premier demi-siècle, 1858-1917), Aix-en-Provence, PUP, 1999.

5. Notons que ces lettrés désirent tous ardemment la souveraineté du Vietnam. Leur opposition pourrait être considérée plutôt comme un partage des tâches. L'historiographie vietnamienne voit les deux Phan comme deux lettrés patriotes l'un et l'autre. 
en 1906 en caractères chinois, traduite immédiatement en français et largement diffusée par la presse de l'époque ${ }^{6}$. Plaidant pour une collaboration loyale avec le pouvoir colonial, il revendique pour les lettrés modernistes le rôle d'intermédiaire légal et légitime entre ce dernier et le peuple. La nouvelle politique coloniale inaugurée officiellement en février 1905, dite politique d'association, semble propice à cette vision.

Indéniablement, les lettrés vietnamiens trouvent une nouvelle source de réflexion dans les "nouveaux écrits " importés des pays voisins. Ces livres présentent des idées occidentales, notamment celles des Lumières; Montesquieu et Rousseau (Mạnh Đức Tư Cưu et Lư Thoa dans la transcription vietnamienne de ces noms en chinois) prennent via ces écrits le statut de véritables nouveaux maîtres à penser. Ces mêmes ouvrages relatent aussi les hauts faits des patriotes et héros politiques occidentaux, en leur donnant valeur d'exemplarité; ils vantent également la modernisation japonaise et rapportent des événements récents, comme le font par exemple Mậu Tuất chính biến (sur la réforme de cent jours en 1898 en Chine), Trung Quốc hồn ("L’Âme chinoise "), ou encore Nhật Bản duy tân sử (" Histoire de la modernisation japonaise »).

Encouragés par ces exemples japonais et chinois, les lettrés se lancent dans une entreprise d'ouverture sans précédent, qui a pour slogan khai dân trí, chấn dân khí, đào tạo nhân tài (" éduquer le peuple, le revigorer, former son élite »). C'est le temps du duy tân (" modernisation »). Mais ce moment est de courte durée; foisonnant d'initiatives inédites, il est malheureusement stoppé brutalement par le pouvoir colonial, effrayé par les manifestations anti-impôt en Annam à partir de mars 1908, puis par la tentative d'empoisonnement de la garnison de Hanoi le 27 juin 1908.

Les recherches effectuées sur ce moment moderniste confirment la réalisation remarquable du premier volet du programme. L'école Đông Kinh Nghĩa Thục (" école de la juste cause ») ouverte à Hanoi en 1907 en est le témoignage le plus célèbre, mais à cette époque sont créées un peu partout d'autres écoles qui accueillent des dizaines et parfois des centaines d'élèves de tous âges ${ }^{7}$.

\footnotetext{
6. Cette lettre est publiée dans le Bulletin de l'École française d'Extrême-Orient, 1907, p. 166-175.

7. Voir notamment Nguyễn Hiến Lê, Đông Kinh Nghĩa Thục, Hanoi, Éd. Van Hoa - Thong Tin, 2002 (première édition en 1958 à Saigon). Dans le Centre, le nombre d'écoles semble approcher la quarantaine et les trois écoles les plus grandes de la province de Quang Nam comptent chacune quelque de 70 à 80 élèves. Voir Trương Chính, Đôi điều khác biệt giữa các nho sĩ Trung quốc với các nhà nho Việt Nam khi tiếp nhận Tân Thư ("Quelques différences dans la réception des Nouveaux Livres chez les lettrés chinois et Vietnamiens »), in Đinh Xuân Lâm (dir.), Tân Thư và xã hội Việt Nam cuối thế kỷ XIX đầu thế kỷ XX ("Les Nouveaux Livres et la société vietnamienne à la fin du XIXe-début XXe siècle »), Hanoi, Éd. Chính Trị Quốc Gia, 1997, p. 189.
} 
Ce qui est moins connu, c'est l'effort d'adaptation de ces lettrés de formation classique à leur environnement économique et leur souci d'y marquer leur emprise. Joignant les actes au discours, ils créent un nombre considérable de sociétés coopératives artisanales, agricoles ou commerciales: Liên Thành, entreprise de fabrication du nước mắm, fondée en 1906, fonctionnera jusqu'en $1975^{8}$; on voit même des mandarins démissionner de leurs postes pour se lancer dans l'entreprenariat. Un exemple illustre bien ce changement de mentalité: envoyés au bagne à Côn Đảo, Huỳnh Thúc Kháng et ses amis montent en 1916, profitant d'une politique plus libérale des autorités, une affaire si rentable qu'ils se retrouvent à la tête du commerce le plus important de l'île, dépassant même les Chinois ${ }^{9}$.

S'il est incontestable que ces transformations culturelles radicales ont eu les " nouveaux écrits " pour déclencheur, il y a probablement lieu de faire la part de raisons endogènes, à étudier dans la profondeur historique: par exemple, les liens entre le milieu lettré et celui des affaires dans le Vietnam précolonial ${ }^{10}$. En s'intéressant aux initiatives concrètes de l'époque moderniste, par exemple, la coopérative fondée par Lê Cơ dès $1904^{11}$, on pourrait mettre à jour des processus déjà à l'œuvre dans le Vietnam précolonial.

Le moment moderniste présente encore une autre particularité. Outre les lettrés, cette sensibilité concerne en effet des milieux très divers et rarement évoqués. Pour l'historiographie vietnamienne, l'école Đông Kinh Nghĩa Thục fut surtout le lieu de ralliement des lettrés patriotes. Or, il est maintenant établi qu'elle réunissait à la fois des lettrés et des nouveaux diplômés dans un projet collectif; des francophones y donnaient des cours de français et de quoc ngu, s'occupaient des relations avec l'administration

8. Trinh Van Thao, "Liên Thành (1906-1975), naissance et transfiguration d'une entreprise moderniste. Essai de sociologie historique sur une expérience entrepreneuriale dans la protoindustrialisation du Vietnam », in Gilles de Gantès et Phuong Ngoc Nguyen (dir.), ouvrage cité, p. 149-162.

9. Huỳnh Thúc Kháng, Huỳnh Thúc Kháng tự truyện (" Autobiographie de Huỳnh Thúc Kháng »), Hué, Éd. Anh Minh, 1963, p. 37-38.

10. Au milieu du XIXe siècle par exemple, Vũ Tông Phan, un des représentants les plus éminents des lettrés du Nord, s'allie avec la famille Bùi, riche propriétaire, en autorisant le mariage de son fils aîné à la fille des Bùi. Cf. Vũ Thế Khôi, «L'association Hướng Thiện des lettrés du temple Ngọc Sơn et l'origine socio-culturelle de Duy Tân et de Đông Kinh Nghĩa Thục », in Gilles de Gantès et Phuong Ngoc Nguyen (dir.), ouvrage cité, p. 98.

11. 100 năm trường Tân học Phú Lâm và nhà thực hành Duy Tân xuất sắc: Lê Cơ (" 100 ans de l'école de nouvelles études Phú Lâm et le praticien moderniste éminent: Lê Cơ »), Quảng Nam, Département de la Culture et de l'Information, 2006. 
coloniale $^{12}$ et diffusaient les idées modernistes dans leur journal Đăng cổ tùng báo (1907) ${ }^{13}$. En Cochinchine, certains bourgeois apparemment assimilés menèrent des actions modernistes, tel Gilbert Chiếu, citoyen français et patriote vietnamien ${ }^{14}$. Fait remarquable, des femmes participèrent à ces entreprises de manière tout à fait résolue ${ }^{15}$. Et si l'on conçoit l'esprit moderniste comme la capacité à s’adapter à un environnement socio-économique nouveau, on peut, à coup sûr, y rapporter aussi les initiatives de ces villageois illettrés qui s'employèrent à maîtriser le changement social à leur avantage, tels ces paysans des environs de Vinh faisant le choix d'aller travailler dans une usine de chemin de fer ${ }^{16}$.

La conjonction de ces dispositions à agir chez plusieurs groupes sociaux aide seule à comprendre l'ampleur des actions modernistes et la rapidité de leur développement. En ce début $d u x^{\mathrm{e}}$ siècle, il y a accord général sur la nécessité du changement: telle est la réalité. Mais qui sont ceux qui ont eu l'accès aux nouvelles idées et qui contribuent à les diffuser largement dans la société?

\section{Acteurs, moments, lieux, moyens de communication}

Il faut avoir à l'esprit la grande complexité de la situation linguistique dans le Vietnam colonial au début du $x^{e}$ siècle. De la Cochinchine au Tonkin, trois langues et trois écritures coexistent pendant quelques décennies: le français est la langue officielle de l'administration coloniale, le peuple vietnamien parle sa langue qui s'écrit en caractères nôm ou en écriture romanisée quoc ngu, la Cour et le mandarinat continuent à utiliser les caractères chinois; les lettrés travaillent les classiques confucéens pour se préparer aux concours triennaux qui ne disparaissent

\footnotetext{
12.Nguyễn Hiến Lê, Đông Kinh Nghĩa Thục, Phuong Ngoc Nguyen, « La Société d'enseignement mutuel du Tonkin (Hội Trí Tri, 1892-1946) - une autre version de l'action moderniste », in Gilles de Gantès et Phuong Ngoc Nguyen (dir.), ouvrage cité, p. 223-238.

13.Pour les historiens vietnamiens, ce journal est « progressiste ». Voir Đỗ Quang Hưng, Lịch sử báo chí Việt Nam (1865-1945) [« Histoire du journalisme au Vietnam »], Éd. Dai Hoc Quoc Gia, Hanoi, 2000, p. 44.

14. Brocheux Pierre, « Note sur Gilbert Chiêu (1867-1919), citoyen français et patriote vietnamien », Approche-Asie, n 11, 1992, p. 72-81.

15. Bùi Trân Phượng, «Les femmes dans le mouvement de la modernisation vietnamienne au début du xxe siècle : les germes d'une émergence », in Gilles de Gantès et Phuong Ngoc Nguyen (dir.), ouvrage cité, p. 163-176.

16. David Del Testa, «S'adapter pour ne pas être expulsé: les manifestations paysannes de Vinh en 1905 », in Gilles de Gantès et Phuong Ngoc Nguyen (dir.), ouvrage cité, p. 135-148.
} 
définitivement du paysage qu'en 1919. Ce n’est que dans les années 1920, sous l'effet du règlement général de l'Instruction publique adopté en 1917, qu'on voit arriver à l'âge adulte une nouvelle génération francophone formée entièrement à l'école franco-indigène ${ }^{17}$.

Pendant cette période de la transition linguistique, rares sont ceux qui maîtrisent les trois écritures. Notons que les lectures en chinois et en français ne se rejoignent pas toujours: l'orientaliste Noël Péri, lors du concours mandarinal de 1910, constate que le candidat confronté à la lecture d'un texte français parlant de Montesquieu ignore souvent qu'il s'agit du célèbre Mạnh Đức Tư Cưu des réformistes chinois ${ }^{18}$. Ce n’est qu'un peu plus tard, dans la Đông Dương tạp chí (" Revue indochinoise », 1913-1919) que l'on pourra découvrir les noms de personnalités occidentales célèbres écrits en quoc ngu, avec leurs noms en caractères chinois et en français ${ }^{19}$.

Les gens ordinaires n'ont pas encore accès aux textes écrits, loin s'en faut. Le nombre de personnes directement impliquées dans le processus de transfert reste ainsi, logiquement, bien faible. On peut, en fait, identifier quatre groupes sociaux qui ont joué un rôle le plus actif dans la réception, la sélection et la transmission des idées occidentales.

Le premier groupe est celui des lettrés non mandarins. Avec des figures emblématiques telles que le vice-docteur Phan Châu Trinh et les docteurs Huỳnh Thúc Kháng et Ngô Đức Kế, ils sont entrés dans l'histoire sous la bannière collective des " lettrés patriotes " ayant refusé les profits de la carrière mandarinale et, pour plusieurs d'entre eux, enduré les prisons coloniales. C'est ce groupe qui est à l'honneur dans l'historiographie vietnamienne. Avec la fin des concours, d'autres lettrés, comme le célèbre poète Tản Đà, s'inscriront dans le sillage de ce groupe et découvriront à leur tour ces Lumières qui éclaireront leur vie d'un nouvel éclat ${ }^{20}$.

Le deuxième groupe est celui des lettrés mandarins ${ }^{21}$. Contrairement aux premiers, ils œuvrent en faveur de la modernisation tout en restant

17. Trinh Van Thao, École française en Indochine, Paris, Karthala, 1995.

18. Noël Péri, Rapport au gouvernement général à propos du concours de doctorat de 1970, Archives EFEO, carton XVI, dossier 20 (participation à l'organisation des examens en langues orientales).

19. Sur la presse vietnamienne en quoc ngu, voir Phạm Thế Ngũ, Việt Nam văn học sử giản ước tân biên (tập III - Văn học hiện đại 1862-1945 (" Histoire de la littérature vietnamienne », vol. 3, 1862 à 1945), Saigon, Éd. Dai Nam, 1965.

20. Phuong Ngoc Nguyen, «Tản Đà (1889-1939) - un lettré rêveur de I'Occident », revue Moussons, n² 24, décembre 2014.

21. Emmanuel Poisson, Mandarins et subalternes au Nord du Vietnam, une bureaucratie à l'épreuve (1820-1918), Paris, Maisonneuve \& Larose, 2004. 
à leur poste. Trần Tán Bình, de formation classique et non francophone, membre de la première mission permanente indochinoise venue en France pour étudier la civilisation française ${ }^{22}$, donne en 1907 des conférences retentissantes en faveur de la modernisation ${ }^{23}$. Une autre personnalité marquante est Thân Trọng Huề, membre de la famille royale et ancien élève de l'école coloniale ${ }^{24}$; il se prononce en faveur de la modernisation du système d'enseignement et de la société. On peut encore citer Đoàn Triển, qui milite pour les nouvelles études, ou Nghiêm Xuân Quảng qui démissionne pour créer une entreprise artisanale. Malgré l'image répandue du mandarin "véreux ", pour toute une partie du mandarinat, "la modernisation est une préoccupation majeure $»^{25}$.

Le troisième groupe représente une catégorie sociale nouvelle, celle des " nouveaux diplômés ", désignation générique des premiers élèves francophones formés pour remplir les fonctions subalternes indispensables au fonctionnement de l'administration coloniale. Contrairement à une idée reçue sur ces secrétaires «buvant du champagne le soir, du lait le matin " (tối rượu sâm banh sáng sũa bò), on peut voir une association comme la Société d'enseignement mutuel du Tonkin (SEM du Tonkin) ou un individu comme Nguyễn Văn Vĩnh s'engager en faveur des actions modernistes ${ }^{26}$, d'ailleurs souvent en collaboration avec les lettrés de formation classique. Encore peu développé au début du xx $\mathrm{x}^{\mathrm{e}}$ siècle, ce groupe préfigure l'apparition des intellectuels francophones de la génération suivante.

Le quatrième groupe est le moins bien connu. Il regroupe les premiers entrepreneurs partisans de la modernisation. Trần Chánh Chiếu, dit Gilbert Chiếu (cf. supra), est actif en Cochinchine; Bạch Thái Bưởi, secrétaire dans une société française puis dans l'administration tonkinoise,

22. Pasquel Rageau Christiane, "Récits de voyage de "mandarins" vietnamiens et cambodgiens en France (1906-1907) ", in Récits de voyage des Asiatiques (Genres, mentalités, conception de l'espace), Paris, EFEO, 1996, p. 385-405.

23. Sur une de ses conférences : BEFEO, 1907, p. 155-166. Sur Trần Tán Bình (1868-1937), on peut consulter sa biographie sur http://tronglang.com, site tenu par un de ses descendants.

24. Sur Thân Trọng Huề (1869-1925), voir Emmanuel Poisson, 2004, 189-190. Une notice nécrologique est fournie par Pasquier Pierre, son camarade à l'école coloniale : «Thân Trọng Huề », BAVH, 1925, p. 211-215.

25. Emmanuel Poisson, Mandarins..., p. 189. Soulignons que Huỳnh Thúc Kháng, une des figures emblématiques des lettrés patriotes, évoque dans ses mémoires la figure de Thân Trọng Huề comme un auteur moderniste (Huỳnh Thúc Kháng, Autobiographie..., p. 26).

26. Sur le rôle de la SEM du Tonkin et d'autres SEM dans la vie culturelle, cf. Phuong Ngoc Nguyen, À l'origine de l'anthropologie au Vietnam, PUP, Aix-en-Provence, 2012. Sur Nguyễn Văn Vĩnh, cf. Emmanuelle Affidi, Đông Dương Tạp Chi (1973-1919), une tentative de diffusion du discours et de la science de l'Occident au Tonkin: l'interculturalité, un enjeu colonial entre savoir et pouvoir (1906-1936), thèse, Paris VII, 2006. 
démissionne après avoir fait le voyage de Bordeaux pour l'exposition de 1895 et s'impose comme l'un des plus grands entrepreneurs vietnamiens. Possédant une bibliothèque riche en "nouveaux écrits ", il la met à disposition de ses amis lettrés ${ }^{27}$. L'étude de ces hommes d'affaires soucieux de se rendre utiles à la société pourrait fournir des éclairages inédits sur le changement qui s'opère à l'époque coloniale.

Ces groupes sociaux, qui ont accès aux informations et qui en effectuent la sélection selon leur compréhension et leurs intérêts propres, participent à la transmission des idées avec la légitimité que leur confèrent leurs positions respectives dans la société à ce moment donné.

Dans ce processus, il convient de garder à l'esprit l'existence d'intermédiaires occasionnels: des Chinois (propriétaires de bibliothèque ou de librairie), des Français (membres de la Ligue des droits de l'homme, par exemple). Il faut également se poser la question des liens personnels noués entre des catholiques et des non-catholiques au Vietnam, malgré l'isolement concevable en situation coloniale de la communauté catholique vietnamienne par rapport à d'autres fractions dans la société.

Un autre sujet d'interrogation est la chronologie même de l'introduction des idées occidentales au Vietnam. Bien que le déroulement général soit connu, il y a des moments dont la nature et l'importance n'apparaissent qu'à travers la connaissance plus ou moins précise que l'on peut prendre d'histoires individuelles et de trajectoires singulières. Influencé par la restauration de Meiji au Japon, le lettré catholique Nguyễn Trường Tộ proposa ainsi à la cour de Hué des idées de réforme de nature à permettre de résister à la conquête française ${ }^{28}$. Grâce à quelques lettrés dont Nguyễn Lộ Trạch, auteur de Thiên hạ đại thế luận ("Commentaires sur l'état du monde"), ses écrits furent conservés et exercèrent quelque influence dans le milieu très confidentiel du haut mandarinat ${ }^{29}$ : la capitale impériale, endroit réputé le plus conservateur, fut ainsi, paradoxalement, le lieu où l'on pouvait, fort discrètement, accéder à des appels au changement. On peut en citer quelques exemples à l'appui. Phan Bội Châu rencontre à Hué, aux alentours de 1900, Nguyễn Thượng Hiền qui « conservait en secret les écrits de Nguyễn Lộ Trạch qu'il n’avait jamais montré à personne avant [lui] ${ }^{30}$ ». Ngô Đức Kế,

27. Đặng Phong, "Vietnam au XXe siècle, de Duy Tân au Đổi Mới », in Gilles de Gantès et Phuong Ngoc Nguyen (dir.), ouvrage cité, p. 276.

28. Cf. Nguyễn Bá Cần, Nguyễn Trường Tộ - con người và di thảo. Nxb TP. Hồ Chí Minh, 2002.

29. Đào Duy Anh, «Les grandes familles de l'Annam. S.E. Tran-Tiên-Thanh », Bulletin des Amis du Vieux Huế, n² 2, avril - juin 1944, p. 91-158.

30. Phan Bội Châu niên biểu ("Phan Bội Châu - chronologie »), Éd. Van Nghe TPHCM, Ho Chi Minh-ville, 2001, p. 59. 
reçu docteur en 1901, refuse d'intégrer le mandarinat, sensibilisé qu'il est par les lectures auxquelles il s'est livré dans la célèbre bibliothèque Long Cương de Cao Xuân Dục ${ }^{31}$. Il semble même que le roi Thành Thái, qui sera déposé par les autorités coloniales en 1907, avait une bibliothèque secrète riche en "nouveaux écrits " qu'il prêtait de temps en temps à quelques personnes de confiance ${ }^{32}$. Phan Châu Trinh, reçu vice-docteur en 1901, se soucie moins d'être présent à son bureau au ministère des Rites que d'aller " lire les " nouveaux écrits ", discuter avec Đào Nguyên Phổ et Thân Trọng Huề ${ }^{33}$ ». Quant à Huỳnh Thúc Kháng, c'est seulement à l'âge de vingt-neuf ans et après avoir été reçu au concours de 1904 qu'il a l'occasion de lire des " nouveaux écrits " chez Đào Nguyên Phổ ${ }^{34}$. Convaincu par ces lectures, il prend la voie de la modernisation en refusant la carrière toute tracée: «Depuis toutes ces années, le vœu de mon père était seulement la réussite au grand concours, j'atteins donc l'objectif; quant à faire carrière de mandarin, cela n'était pas mon souhait. Pour cette raison, après être reçu docteur, je prétextai une maladie pour rester chez moi $^{35}$. "

En 1905, un voyage singulier eut lieu. Les docteurs Huỳnh Thúc Kháng et Trần Quý Cáp, ainsi que Phan Châu Trinh qui avait démissionné de son poste, partirent pour le Sud du pays, afin de le visiter. De passage dans une petite ville où l'on organisait un examen blanc pour les candidats aux concours, les trois amis s'inscrivirent sous de faux noms et rédigèrent un poème et un texte de prose signés Đào Mộng Giác (« Đào qui se réveille du rêve ") dans lesquels ils appelaient les lettrés à abandonner les concours pour se rendre utiles au pays. Immédiatement diffusés, largement, ces textes rencontrèrent un écho favorable dans le milieu lettré déjà impressionné par la victoire du Japon sur la Russie, qui eut un grand retentissement dans le Vietnam colonial $^{36}$. Bien évidemment, la lecture ne signifie pas la compréhension ni l'adhésion, mais cet appel de trois grands lauréats semble être bien entendu comme en témoignent les nombreuses créations d'écoles et de sociétés agricoles, artisanales et commerciales.

\footnotetext{
31. Ngô Đức Kế. Cuộc đời và tác phẩm ("Ngô Đức Kế - vie et œuvre "), Sở văn hóa thể thao và du lịch Hà Tĩnh, 2008. p. 447.

32. Ibid., p. 467

33. Phan Châu Trinh, về tác gia và tác phẩm ("Phan Châu Trinh - sur l'auteur et son œuvre »), Hanoi, Éd. Giáo dục, 2007. Chương Thâu, et Hải Yến Trần (dir.), p. 56.

34. Huỳnh Thúc Kháng, op. cit., p. 26.

35. Huỳnh Thúc Kháng, Autobiographie..., p. 26-27.

36. Trinh Van Thao, "La perception vietnamienne de la conjoncture de 1905 », Études Indochinoises III, IPHOM, $\mathrm{n}^{\circ} 25$.
} 
$\mathrm{Au}$ début $\mathrm{du} \mathrm{xx}^{\mathrm{e}}$ siècle, les livres en français sont rares, du fait de l'éloignement géographique et de leur coût élevé. La possibilité d'y accéder est offerte par un séjour en France dans le cadre des études (cas de Thân Trọng Huề de 1888 à 1895) ou d'une mission (cas de Nguyễn Văn Vĩnh envoyé à l'exposition coloniale de Marseille en 1906). En Cochinchine, où les contacts sont plus précoces, il est plus aisé d'en acheter ou d'en lire, mais cela reste limité. C'est donc, sans conteste, essentiellement par l'intermédiaire des écrits en chinois que les Vietnamiens sont entrés en contact avec les philosophes occidentaux. Les ports internationaux sont des points de contact avec l'étranger: Hué profite de sa situation à une centaine de kilomètres de Tourane et Hanoi bénéficie de Haiphong, tandis que Saigon, port le plus important d'Indochine, voit arriver les navires européens qui remontent le Mékong. Les bibliothèques privées les plus importantes se trouvent logiquement dans ces centres urbains. Mais ce qui est remarquable, c'est l'importance de certaines bibliothèques installées dans des villages et jouissant d'une grande réputation, comme celles de la famille de Ngô Đức Kế, de la famille de Đặng Thai Mai ou de la famille de Đặng Xuân Bảng. Ce n’est pas un hasard: des actions entreprises par les lettrés modernistes, la création d'une bibliothèque collective est souvent la première.

On peut distinguer les différentes étapes de la diffusion des idées nouvelles. Au début, la lecture, solitaire, s'effectue le plus souvent dans un cadre confidentiel. D’après le témoignage de Huỳnh Thúc Kháng, ce n’est qu'autour de 1903-1904 que les idées commencent à circuler plus ouvertement. Il faut garder à l'esprit que les rencontres entre lettrés sont autant occasions pour discuter en confiance et diffuser les idées nouvelles dans un cadre amical. On sait que les lettrés aiment se réunir pour faire de la poésie et se divertir aux jeux raffinés des belles lettres ${ }^{37}$. Mais les sociabilités lettrées ne se limitent pas à ces divertissements: les écoles ont par exemple pour coutume d'inviter des lettrés réputés à venir commenter des textes, dont des compositions d'élèves ${ }^{38}$. À la différence de leurs aînés qui visaient la réforme par le haut en présentant des suppliques au souverain, au début $\mathrm{du} \mathrm{xx}^{\mathrm{e}}$ siècle les lettrés modernistes cherchent d'abord à convaincre leurs pairs lettrés.

Les grands rassemblements pendant les périodes de concours favorisent d'ailleurs la transmission des idées. Quand des milliers de lettrés se rassemblent au même moment dans un même lieu, une nouvelle peut se

37. Nguyễn Tuân, Vang bóng một thời ("Echos et reflets d'un temps »), Hanoi, 1940.

38. Emmanuel Poisson, op. cit., p. 189. 
propager à une grande vitesse. Il faut également garder à l'esprit qu'autour des lettrés gravite tout un monde de serviteurs (qui portent leurs équipements) et de marchands (de papier, d'encre, etc.). Par ces relais, une information peut se diffuser dans d'autres groupes sociaux. Dans une société ignorant encore la presse écrite, le rôle de la báo miệng (presse orale) est essentiel. Sa vitesse et son efficacité peuvent être redoutables. Ce n'est certainement pas un hasard si l'école Đông Kinh Nghĩa Thục, outre la diffusion de textes écrits, fait délibérément le choix de la poésie pour promouvoir les nouvelles idées par la transmission orale ${ }^{39}$. Les lettrés modernistes vont jusqu'à se rendre aux pagodes et aux temples pour atteindre la masse des fidèles qui s’y réunit tous les premiers et quinzièmes jours du mois lunaire. C'est au temple Ngọc Sơn, en plein centre de Hanoi, que les lettrés de Đông Kinh Nghĩa Thục font leur première sortie publique. Outre ces lieux de culte, les marchés sont également des lieux où les nouvelles et les actualités se transmettent.

On reste admiratif devant le souci exceptionnel qu'ont ces lettrés, "chefs naturels" pour reprendre l'expression de Nguyen The Anh, d'expliquer et de diffuser les idées nouvelles: le plus largement possible, par un moyen approprié (la transmission orale) et avec le renfort de leur présence dans les lieux publics. L'engagement de ces grands lauréats des concours, investis d'une légitimité reconnue, exerça indéniablement un pouvoir de conviction. Il n'est pas étonnant que ces lettrés non mandarins soient restés dans la mémoire collective comme les acteurs de la modernisation.

Dans les années 1906-1907, on voit apparaître une nouvelle forme de communication: les causeries ou conférences. L'école Đông Kinh Nghĩa Thục, comme d'ailleurs la SEM du Tonkin, invite des grands noms qui attirent un public nombreux. Le phénomène s'étend même en dehors de Hanoi. Les conférences, en province, sont parfois l'occasion de véritables triomphes des nouvelles idées: en 1907, Trần Tán Bình fait une conférence le 6 mars à Nam Định devant 1000 personnes, le 7 mars à Lạc Quân — 1500 personnes - et le 9 mars à Núi Gối en présence de 6000 personnes $^{40}$.

39.Le Quốc văn tập đọc («Livre de lecture de la littérature nationale ») comprend dix-neuf poèmes écrits en quoc ngu qui sont autant des arguments "pour apprendre le quoc ngu », " pour aimer la patrie », " pour faire des études à l'étranger », pour encourager à « lire les journaux », etc., parfois présentés sous forme des paroles « de la femme au mari », « de la mère à l'enfant », « adressées à la jeunesse », « adressées aux fumeurs d'opium », aux « alcooliques » et même aux " amateurs de femmes », etc.

40. Đăng cổ tùng báo, 28 mars 1907, p. 14. 
C'est une époque d'innovations importantes. L'édition et la presse écrite n'étaient pas inconnues, mais le Đăng cổ tùng báo (1907) dirigé par Nguyễn Văn Vĩnh s’impose comme le premier journal en quoc ngu à avoir milité au Tonkin en faveur de la modernisation. Le comité de rédaction de l'école Đông Kinh Nghĩa Thục fournit en manuels non seulement cette dernière, mais aussi d'autres écoles du Nord et du Centre, augmentant sensiblement le public touché. La revue Đông dương tạp chí de Nguyễn Văn Vĩnh œuvre pour la modernisation au moins jusqu'en 1915, quand il deviendra impossible de critiquer le mandarinat dans le contexte de la Première Guerre mondiale.

Reste un autre moyen de communication: il s'agit du roman, dont les penseurs japonais et chinois font grand cas, car ils l'estiment le plus approprié pour diffuser les nouvelles idées au sein des masses populaires. Les lettrés vietnamiens en connaissent la force, sous la plume de Liang Qichao essentiellement, et certains ont l'occasion de lire Giai nhân kỳ ngộ ("Rencontre merveilleuse avec des beautés »), un célèbre roman japonais racontant les aventures d'un Japonais en Occident ${ }^{41}$. Phan Châu Trinh entreprendra même sa traduction en vietnamien pendant son exil en France ${ }^{42}$. Au Vietnam, il faudra attendre un peu pour voir paraître le premier roman de ce type: dans Giấc mộng con ("Le Petit rêve ") écrit en 1916 et publié en 1917, Tản Đà relate le voyage imaginaire d'un jeune homme du pays d'Annam autour du monde et déclare vouloir jouer le rôle d'un "philosophe oriental " au service de son peuple.

Mais que retient-on au Vietnam de ces lectures diverses?

\section{Quelles Lumières?}

Dans les études effectuées au Vietnam sur la période, l'attention est essentiellement orientée sur la poésie et la prose patriotes ou révolutionnaires. Dans la perspective adoptée ici, nous prenons en compte des productions écrites ou orales de tous les autres acteurs de la modernisation: conférences de mandarins, articles de presse de nouveaux diplômés, mais aussi le roman Le Petit rêve qui, malgré sa parution tardive, exprime bien les représentations partagées en milieu lettré.

Il est clair que ce qui est retenu en premier lieu par les lettrés modernistes, dans leur lecture des nouveaux livres, c'est le patriotisme. Les

41. Il s'agit du roman de Tôkai Sanshi intitulé Kajin no Kigū publié en plusieurs volumes de 1885 à 1897 .

42. Phan Châu Trinh, 2007. Voir également Vĩnh Sính, Việt Nam Nhật Bản giao lưu văn hóa ("Échanges culturels entre le Japon et le Vietnam »), Ho Chi Minh-ville, Éd. Văn Nghệ, 2001. 
notions de patrie (tổ quốc) et de peuple (dân) permettent de reconfigurer le sentiment patriotique, qui n'est plus la fidélité au souverain (trung). Un indice en est la popularité extraordinaire du livre de Liang Qichao, Trois grands héros italiens, connu au Vietnam sous le titre Ý Đại Lợi kiến quốc tam kiệt truyện. Mazzini (Mã Chi Nê en transcription sino-vietnamienne) est le héros de Phan Bội Châu et Phan Châu Trinh, les deux leaders de l'élite lettrée. Phan Châu Trinh va jusqu'à proclamer son admiration pour le patriote italien en adoptant comme pseudonyme Hy Mã (suivant Mazzini). Ou encore: Tỉ-tư-mạch (Bismarck), Cách-lan-tư-đốn (Gladstone), Qua-đặc (Jeanne d'Arc), mais aussi La-lan ( $\mathrm{M}^{\text {me }}$ Roland) sont réunis pour leur amour de la patrie et leur esprit révolutionnaire dans un poème qui prend la forme des paroles qu'une jeune épouse adresse à son mari pour l'exhorter à se mettre au service de la patrie, en suivant l'exemple de ces héros des pays étrangers ${ }^{43}$. On peut s'étonner de la présence des femmes dans cette littérature. Cela fait sans doute partie de la rhétorique: si même une femme est consciente de l'œuvre à accomplir, alors un homme se doit de la réaliser afin d'être digne de son statut. Dans la réalité, non seulement les femmes prennent une part active dans les actions modernistes comme il est dit plus haut, mais les lettrés, Phan Bội Châu en particulier, manifestent à leur égard une attitude beaucoup plus égalitaire et respectueuse que les « nouveaux diplômés » dans leur ensemble.

Prenant connaissance des idées de penseurs occidentaux, les lettrés vietnamiens semblent particulièrement marqués par les notions d'évolution, de concurrence et de sélection. Dans sa lettre ouverte déjà citée, Phan Châu Trinh prend l'exemple des Peaux-Rouges pour montrer qu'il est conscient que l'extinction d'une " race " n'est pas une vue de l'esprit et attester qu'il envisage la collaboration avec le gouvernement colonial d'une façon tout à fait consciente et loyale: il est donc vital d'apprendre et d'évoluer pour résister à la concurrence. C'est dans ce contexte de prise de conscience d'une possible disparition qu'il faut comprendre le fervent encouragement donné aux études, aux affaires, et en général tout cet intérêt pour les divers aspects de la civilisation occidentale, du point de vue technique et économique, mais aussi de celui de l'organisation sociale et des institutions politiques. Il est évident que l'Occident, par sa vitalité même, est un modèle à suivre. L'ouvrage programmatique Văn minh tân học sách (" Nouvelle étude de la civilisation ») fait l'éloge de son organisation:

43. Đặng Thai Mai, Văn tho cách mạng Việt Nam đầu thế kỷ XX [ [ La littérature révolutionnaire du Vietnam au début du XXe siècle »], 1961, p. 199. 
Si l'on considère les pays occidentaux: en haut il y a une Assemblée pour faire appliquer les lois, en bas il y a la presse comme moyen d'expression populaire. Parmi les œuvres philosophiques il y a le Contrat social de Rousseau, L'Évolutionnisme de Spencer, L'Esprit des lois de Montesquieu. En général, que ce soit les conférences ou la poésie, tout sert à développer l'amour de la patrie, du peuple ${ }^{44}$.

La grande découverte est celle de l'existence d'une autre forme étatique (la république), d'une autre forme d'organisation politique (la démocratie) et d'un autre principe de souveraineté (le peuple). Les notions du dân quyền (droits du peuple), dân ước (contrat du peuple), pháp luật (lois) - tout un groupe de mots empruntés au chinois - font leur entrée dans la langue et dans les esprits vietnamiens. Parmi les soixante-dix-neuf textes du Livre de lecture pour le peuple diffusé par Đông Kinh Nghĩa Thục, le thème de la vie en société est le plus important. Pour exemple, ces titres parlants: "Les citoyens doivent savoir l'importance de la politique ", " À propos des droits et des responsabilités ", "À propos des lois ", etc. Dans sa conférence, Trần Tán Bình livre un récit d’une grande sincérité de sa découverte de l'Occident:

Lorsque pour la première fois je lus, dans une traduction chinoise d'un livre européen, le mot " République ", je ne pouvais concevoir comment un État pouvait subsister sans Roi qui ordonne, sans « pères et mères du peuple » qui administrent des coups de rotin à leurs enfants. Ce nom de " domestiques du peuple » que les réformistes chinois donnent aux fonctionnaires sonnait mal à mes oreilles. Comment, me demandais-je, des hommes qui passent des années à étudier pour être reçus aux examens triennaux, peuvent-ils sans injustice ne servir qu'à travailler pour cette vile populace? Comment se peut-il qu'ils n’aient pas une supériorité sur les autres? Les termes "égalité ", "fraternité entre tous les hommes ", choquaient violemment mes oreilles ${ }^{45}$.

C'est cette sincérité qui permet à ce mandarin de parler du changement complet de ses idées après avoir vu les institutions démocratiques en France:

Je suis convaincu maintenant que de par le monde, il y a une nation, et même plusieurs, où la souveraineté réside dans le peuple, où les fonctionnaires, voire le plus grand, le chef de l’État, qui est le président de la République, n'ont qu'un pouvoir exécutif, c'est-à-dire qu'ils ne font autre chose que de faire ce qu’ont décidé les représentants élus par le peuple.

44. Ce texte anonyme en caractères chinois est traduit en vietnamien par Đặng Thai Mai et repris dans Luận về quốc học ("Essai sur les études nationales »), Da Nang, Éd. Da Nang, 1999, p. 159.

45. BEFEO, 1907, p. 160. 
Pour beaucoup de lettrés, dân quyền (droits du peuple) ne signifie nécessairement la démocratie et la république. Le choix vietnamien se penche plutôt vers la monarchie constitutionnelle, en prenant l'exemple japonais. Les lettrés sont d'ailleurs réticents à l'idée du contrat qui leur semble mercantile, car basé sur l'intérêt, et font plutôt référence à une communauté issue des mêmes ancêtres, les rois Hùng légendaires ${ }^{46}$. Mais l'essentiel est qu'on conçoit que le peuple a désormais son mot à dire. Cette idée, inédite dans la société vietnamienne, trouve son application pratique à l'occasion des élections des conseillers vietnamiens à la mairie de Hanoi en 1907. Dans plusieurs numéros, le Đăng cổ tùng báo rend compte des élections et accompagne ses lecteurs dans l'apprentissage de la démocratie. À propos du vote, le journal écrit:

Il faut réfléchir avant de venir sur le choix de la personne pour qui voter, il faut bien choisir une personne intelligente qui a un statut social et qui connaît la loi, c'est bien un choix personnel, personne ne peut vous obliger à faire ce que vous ne voulez pas $^{47}$.

Pour les générations plus jeunes qui maîtrisent le français, cela semble être bien naïf. Malgré les railleries dont les lettrés sont l'objet, qu'en est-il en réalité? Pour essayer d'y voir plus clair, prenons le cas de Rousseau.

\section{Des Rousseau pluriels et divergents}

En Chine, le philosophe est rendu célèbre par l'intermédiaire de Liang Qichao qui souligne l'apport du Contrat social. Dans un dossier d'études sur Rousseau publié en 1901, il présente une courte biographie de Rousseau et expose des notions principales de son œuvre (liberté, égalité, souveraineté du peuple, volonté générale et différentes formes de gouvernement). Dans une lettre à Kang Youwei, Liang Qichao écrit: " Il faut que le peuple chinois prenne conscience que la liberté est attribuée par le Ciel, on ne peut se la laisser ôter par autrui ni y renoncer de soi-même ${ }^{48}$. "

C'est dans les écrits de Liang Qichao que les lettrés vietnamiens découvrent ces notions du droit et de la liberté, d'ailleurs dans un style

46. Depuis 2000, ces rois légendaires sont proclamés « ancêtres » de la nation vietnamienne par le Parti communiste et le gouvernement.

47. Đăng cổ tùng báo, 25 avril 1907, p. 66.

48. Cité par Xiaoling Wang, "Liang Qichao, lecteur de Rousseau», Études Jean-Jacques Rousseau, $n^{\circ} 18,2010-2011$, p. 261. La première traduction de Rousseau en chinois a été réalisée par un Japonais Nakae Chômin au printemps 1898, mais n'a pas eu d'influence. C'est plus tard, par des lettrés en exil au Japon, que le philosophe est mieux connu. II existe plusieurs traductions du japonais, puis du français en 1916 par Ma Junwu. 
flamboyant qui leur communique l'enthousiasme de l'auteur. Liang réalise un colossal travail d'explication et donne une réelle valeur ajoutée aux textes de Rousseau ${ }^{49}$. On sait qu'avec Montesquieu, Rousseau est le philosophe occidental le plus connu au Vietnam au début du xx $\mathrm{x}^{\mathrm{e}}$ siècle. Sa représentation la plus répandue chez le lecteur vietnamien est peutêtre celle qu’offre le poème de Tản Đà intitulé Nhớ ông Lư Thoa ("Penser à monsieur Rousseau ") publié dans la revue Hũu Thanh en 1921 où l'auteur se dit élève du philosophe: Rousseau y est présenté comme le promoteur des droits de l'homme (nhân quyền) et du contrat social (dân ước). Dans le roman Le Petit rêve publié en 1917, on peut également voir l'ombre du philosophe se profiler dans la description d'une société idéale, le Nouveau Monde découvert au milieu des glaces du pôle Nord par les voyageurs dont fait partie le héros. Dans cette petite communauté, tout se fait en commun et par un commun accord, il n'y pas d'argent ni d'oppression, y compris vis-à-vis de la nature et des animaux qui tirent les voitures sans harnachement ${ }^{50}$.

Paradoxalement, dans la revue Đông dương tạp chí des " nouveaux diplômés " francophones, Rousseau est peu présent. De 1913 à 1918, on peut juste dénombrer sept extraits traduits dans la rubrique morceaux choisis : cinq tirés de l'Émile, un de La Nouvelle Héloïse, un des Lettres morales, alors que La Fontaine totalise cinquante textes et Molière environ trente ${ }^{51}$. Les extraits de Rousseau ne sont d'ailleurs pas situés dans leur contexte et comportent des omissions. Deux exemples peuvent en donner une idée. Dans une lettre, "Ma respectable amie " disparaît dans la traduction vietnamienne, et comme la langue vietnamienne ne distingue pas le genre, pour le lecteur vietnamien, la lettre d'une teneur philosophique ne peut être adressée qu'à un homme. Dans le dernier extrait tiré de l'Émile, la phrase "Là je rassemblerais une société, plus choisie que nombreuse, d'amis " n'est pas entière, car il manque la fin qui dit "d'amis aimant le plaisir et s'y connaissant, de femmes qui puissent sortir de leurs fauteuils et se prêter aux jeux champêtres ». En résumé,

49. Plus tard, en 1903 après son voyage aux États-Unis, Liang Qichao critiquera Rousseau et adoptera les thèses du juriste Bluntschli (Marianne Bastid-Bruguière, "Aux origines des conceptions modernes de l'Etat en Chine: la traduction du Quojia lun de J. K. Bluntschli », in Jacques Gernet et Marc Kalinowski (dir.), En suivant la Voie royale. Mélanges offerts en hommage à Léon Vandermeersch, Paris, EFEO, 1997, p. 309-417.)

50. Phuong Ngoc Nguyen, op. cit.

51. Emmanuelle Affidi, «Traduire ou ne pas traduire Rousseau: un dilemme pour Nguyễn Văn Vĩnh dans le Việt Nam des premières années du vingtième siècle », Études Jean-Jacques Rousseau, nº 18, 2010-2011, p. 277-292. 
c’est un Rousseau exclusivement pédagogue qui est présenté au lecteur vietnamien. Il s'agit bien entendu d'un choix délibéré, car il est exclu que le rédacteur en chef, Nguyễn Văn Vĩnh, ait ignoré l'influence de Rousseau sur les révolutionnaires. Cet excellent connaisseur de la langue française, une des figures de la modernisation, semble avoir traduit en vietnamien le Contrat social, sans doute avant 1910.

Dans la revue Nam Phong ("Vent du Sud ») qui domine la vie culturelle vietnamienne de 1917 à 1934, quatre numéros consécutifs, du n 104 au $n^{\circ} 107$, sont consacrés à Rousseau ${ }^{52}$. On pourrait dire qu'il s'agit là d'un traitement de faveur, par rapport à Montesquieu ( $\left.n^{\circ} 107\right)$ et Voltaire ( $\left.n^{\circ} 114\right)$. Cependant, l'intérêt porté au philosophe est étonnamment tardif: en 1926, année de parutions de ces numéros, la revue existe déjà depuis dix ans. Le portrait est certes plus complet que dans Đông dương tạp chí: une biographie de Rousseau (sur dix-neuf pages) est suivie d'une présentation de La Nouvelle Héloise et Émile, puis de la Profession de foi $d u$ vicaire savoyard, et, en dernier lieu, d'une présentation du Contrat social (sur douze colonnes) et des Confessions. Mais Phạm Quỳnh, rédacteur en chef, souligne surtout les comportements déviants de Rousseau. Sur le fond, il affirme que sa pensée n’est pas révolutionnaire (" à tous les pays, [Rousseau] conseille de garder le régime politique ») et cherche par là, manifestement, à désacraliser cette figure emblématique des lettrés.

La même année, le Contrat social dont Nguyễn An Ninh a achevé la traduction du français trois ans avant, en 1923, est publié à Saigon; la capitale de la Cochinchine, en tant que colonie française, jouit d'un statut plus libéral que le Tonkin et l'Annam placés sous le régime du protectorat ${ }^{53}$. Bien qu'incomplète, cette traduction réalisée par un francophone imprégné des idées révolutionnaires eut sans doute un certain écho auprès du public vietnamien, qui put y trouver cette référence directe au fait colonial: « Car jusqu'à nos jours, dans l'humanité, le pouvoir politique n'appartient pas en fin de compte au peuple. " Le fait que dans une même année paraissent ces travaux d'esprit tout opposé, est en soi significatif. Il resterait à étudier les conditions de leur production respective, mais on peut supposer que ce sont en quelque sorte les réponses de différents groupes sociaux, qui se

\footnotetext{
52. Phung Ngoc Kien, « L'expérience de lecture de Rousseau au Vietnam dans le premier quart du XXe siècle », communication présentée à la journée d'études Rousseau et la modernité asiatique organisée le 15 décembre 2012 par le groupe Rousseau-Asie (Centre d'étude de la langue et de la littérature françaises des XVII ${ }^{e}$ et XVIII ${ }^{e}$ siècles) à l'université Paris IV-Sorbonne.

53.Nguyễn Lan Huong, «Rousseau inspirant l'idéal annamite. La traduction du Contrat social en vietnamien par Nguyễn An Ninh », Études Jean-Jacques Rousseau, n 18, 2010-2011, p. 293300.
} 
positionnent différemment par rapport à une nouvelle donne de la situation indochinoise, consécutive à l'arrivée au poste de gouverneur général d'un socialiste, Alexandre Varenne.

Les images de Rousseau présentées au public vietnamien sont donc quelque peu assujetties à l'intérêt de la personne qui réalise l'opération du transfert ou du groupe social que cette personne représente. À première vue, nous sommes d'ailleurs devant un paradoxe: ce n'est pas grâce aux traductions directes du français, mais par l'intermédiaire des textes en chinois que Rousseau est présenté au Vietnam de la manière la plus fidèle. À bien y réfléchir, il est logique que les lettrés modernistes, animés par leur patriotisme, aient trouvé dans les textes du philosophe des résonances à leurs préoccupations, d'ailleurs amplifiées par l'interprétation brillante de leur collègue chinois Liang Qichao. Il est également compréhensible que les "nouveaux diplômés " francophones, même admirateurs de Rousseau, n’aient pu si librement transmettre des idées susceptibles d'ébranler l'ordre colonial.

Les idées des Lumières sont entrées au Vietnam par deux voies distinctes: la voie du Nord via les " nouveaux écrits " en caractères chinois lus par les lettrés, et la voie du Sud via les textes en français lus par les "nouveaux diplômés " de l'école franco-indigène. Pendant le bref moment moderniste, de 1905 à 1908, ces différentes lectures aboutissent globalement à la même compréhension. À partir du moment où le pouvoir colonial durcit sa politique à la suite des manifestations de contestation de l'ordre établi, il devient impossible d'avoir le même discours selon la position qu'on occupe dans la société coloniale.

La contradiction du colonialisme s'avère ainsi manifeste et inévitable: la mission civilisatrice est réussie, mais avec un effet inattendu et non souhaité par les autorités coloniales. En lisant les philosophes des Lumières, des Vietnamiens forment le projet de prendre en main leur destinée, ce qui deviendra réalité avec la génération formée par l'école française et nourrie à la civilisation occidentale. 



\title{
4
}

\section{La catholicité vietnamienne}

\section{Quand se conjuguent préservation de la foi et participation au destin national}

\author{
Pierre Brocheux
}

En 2008-2009, une exposition sur les cultures des minorités ethniques du Vietnam se tint au musée national d'ethnographie de Hanoi, avec une section intitulée "Vivre les sacrements - culture catholique contemporaine au Vietnam "; malgré le fait insolite que les catholiques vietnamiens avaient été classés minorité ethnique, il faut retenir que cela valut « hommage aux valeurs religieuses et culturelles de la communauté catholique "; ce fut un acte de reconnaissance officielle de la catholicité nationale (fidèles, clergé et culture) après quatre siècles de rapports conflictuels.

\section{L'ouverture de l'Asie à l'évangélisation}

Les Vietnamiens avaient déjà accueilli le bouddhisme, le confucianisme et le taoïsme, ensemble de croyances supranaturelles, d'idéologies et de pratiques rituelles d'origine étrangère. Dernier arrivé, le christianisme se fit une place dans ce réceptacle complexe où le bouddhisme, et le confucianisme participaient déjà, depuis quatre siècles, à la structuration des liens sociaux, politiques et culturels, à la légitimation du pouvoir politique dans le royaume Đại Việt ${ }^{1}$.

Dans la conscience commune, on relie l'expansion du christianisme porté par les missionnaires de l'Église catholique romaine au mouvement impulsé par les grandes découvertes $\mathrm{du} \mathrm{xvI}^{\mathrm{e}}$ siècle et de la mise en relations transocéaniques de l'Europe et des autres continents. Mais il ne faut

1. Au Đại Việt, le bouddhisme eut une position hégémonique du XI ${ }^{\mathrm{e}}$ au XIII ${ }^{\mathrm{e}}$ siècle, mais à partir du XIVe siècle, le confucianisme commença à s'imposer dans l'éducation et le gouvernement. 
pas oublier que, s'agissant de l'Asie orientale et méridionale, ces relations sont antérieures et remontent au XIv siècle. L'Église catholique n'eut pas à attendre les prises de possession européennes aux Amériques pour enregistrer et penser le fait qu'il existait des peuples et des croyances complètement étrangers à celles que ses missionnaires venaient prêcher.

Pour utiliser le vocabulaire militaire, il y a la stratégie et la tactique. L'Église avait comme but stratégique la christianisation des populations dites païennes, la tactique ou méthode pour parvenir à ce but dépendait du degré d'évolution culturelle de ces peuples: soit la table rase soit l'adaptation. Le jésuite portugais José de $\operatorname{Acosta}^{2}$ se fit le défenseur de l'adaptation au terrain ultramarin où les missionnaires se trouvèrent confrontés à des sociétés évoluées (possédant une écriture, une littérature très anciennes, des coutumes et mœurs raffinées, une tradition artistique, une organisation politique et religieuse aussi avancées que celles d'Europe).

Les jésuites Robert de Nobili en Inde, Alexandre de Valignano au Japon et Matteo Ricci en Chine poussèrent les tentatives d'adaptation assez loin pour être suspectés et condamnés d'avoir enfreint l'orthodoxie de l'Église romaine. Les autres missionnaires, jésuites, franciscains ou dominicains, se soumirent aux directives de Rome tout en adoptant une démarche empirique que leur imposaient les contraintes des traditions locales.

\section{Implantation au Đại Việt}

L'interdiction du christianisme dans l'Empire chinois et l'ostracisme qui frappe les chrétiens au Japon et aboutit à leur expulsion en 1637 poussent les jésuites vers les rivages du royaume du Sud, le Đại Việt; ils débarquent au Đàng Ngoài (Tonkin) en $1626^{3}$. Le moment est déterminant à deux points de vue.

D'une part, le christianisme ne faisait que rejoindre, si l'on peut dire, le mouvement général d'irrésistible expansion dans la région des religions de Salut $\mathrm{au} \mathrm{xv}^{\mathrm{e}}$ siècle (le bouddhisme sur le continent et l'islam dans l'Insulinde).

Mais, d'autre part, il débarquait alors même que s'imposait aux seigneurs Trịnh la nécessité de renforcer par un pouvoir symbolique le

2. De Procuranda Indorum Salute, Madrid, 1588, cité dans Histoire du Christianisme, t.8 le temps des confessions (1530-1620), ch. IX, p. 815, Paris, Desclée, 1992.

3. 3. Le royaume était divisé en deux entités, de part et d'autre du fleuve Sông Giang: Đàng Ngoài et Đàng Trong que les missionnaires appelèrent respectivement Tonkin et Cochinchine. La dynastie des Lê était toujours sur le trône mais les deux grandes maisons seigneuriales Trịnh au Nord et Nguyễn au Sud exercaient le pouvoir réel. 
pouvoir de fait qu'ils exerçaient aux dépens de la dynastie légitime des Lê; rôle symbolique habituellement confié par les seigneurs viêt comme par les empereurs chinois au confucianisme, idéologie d'ordre, de continuité et de stabilité via le culte des ancêtres, ciment d'unité en même temps que symbole de la stricte hiérarchisation de la société. Le confucianisme qui n'est pas une religion à proprement parler, encadre la société et légitime le pouvoir du souverain. C'est précisément ce corpus de rites, dont le culte des ancêtres est la clé de voûte, que l'église catholique estimait incompatible avec ses croyances fondamentales et rites attachés ${ }^{4}$.

D'où un contexte à la fois favorable et défavorable à une implantation du christianisme: si le peuple victime de ce qu'on devait appeler la guerre de Cinquante Ans entre les grandes maisons seigneuriales était en attente d'une espérance de Salut, en revanche, vu les exigences doctrinales du christianisme, la classe des lettrés fonctionnaires porteurs de l'idéologie confucéenne d'apparition contemporaine (1637 et 1640 furent les années de concours de recrutement) ne pouvait que s'opposer à la nouvelle religion et au clergé qui la prêchait.

Le favorable tendit à l'emporter sur le défavorable, dans la mesure où les seigneurs avaient besoin des services des marchands européens (principalement des Portugais); ces derniers servirent de couverture aux prêtres missionnaires, qui jouirent d'une relative liberté religieuse entre 1724 et 1773. En dépit des fluctuations politiques locales mais aussi des querelles entre les congrégations missionnaires, notamment entre les jésuites et les missions étrangères de Paris, la chrétienté catholique progressa: de 83500 en vers la fin du xvI ${ }^{\mathrm{e}}$ siècle le nombre de convertis passa à 140000 en 1707 et à 275000 en 1766. C'est durant cette période que les jésuites inventèrent le quốc ngư, transcription latinisée de la langue viêt ${ }^{5}$ : un instrument d'évangélisation qui devait se transformer trois siècles plus tard en outil de la modernisation culturelle et politique du Vietnam.

\section{Des persécutions à l'hypothèque coloniale}

Le $\mathrm{XIX}^{\mathrm{e}}$ siècle s'ouvrit avec la réunification territoriale et politique du royaume, accompagnée d'une restauration monarchique absolutiste,

\footnotetext{
4. J. Gernet, Chine et Christianisme. Action et réaction, Paris, Gallimard, 1982.

5. L'invention est attribuée au jésuite français Alexandre de Rhodes, fréquemment invoqué dans l'argumentaire colonial français alors qu'il était originaire du Comtat Venaissin et donc sujet du pape. En réalité, il fut initié à la langue du pays par les jésuites portugais déjà sur place; il fit partie, assurément, de ceux qui mirent au point le quốc ngử, mais il n'en fut pas l'inventeur. Voir Roland Jacques, « Le Portugal et la romanisation de la langue vietnamienne. Faut-il réécrire l'histoire? », Revue française d'Histoire d'Outre-mer 1998/1, p. 21-54.
} 
contraignante, à la mesure des déchirures causées par les guerres intestines de plus d'un siècle. Le christianisme en subit les effets négatifs. On proclama son interdiction et des persécutions eurent lieu pour des raisons essentiellement politiques: la religion chrétienne mettait en péril la restauration monarchique en plaçant Dieu, en la personne du Christ, audessus du souverain viêt qui, lui, avait reçu le mandat du Ciel (une notion qui relève de la cosmologie chinoise). Il faut ajouter que les prêtres catholiques faisaient figure de concurrents des lettrés qui se considéraient comme les pères et mères du peuple. Le rejet brutal du christianisme fut le prétexte de l'intervention militaire de la France, en 1858.

En conséquence, la catholicité vietnamienne subit les effets de l'amalgame entre religion chrétienne et domination étrangère.

L'histoire eût-elle pu évoluer autrement? L'aide militaire et diplomatique apportée par les Français à Nguyễn Phúc Ảnh, sanctionnée par un traité en 1787, fut probablement décisive et lui permit de vaincre les Tây Sơn, puis de s'imposer finalement comme empereur, ouvrant la dynastie des Nguyễn sous le nom de Gia Long (1802). En retour de cet appui, l'évêque d'Adran Pierre Pigneau de Béhaine, auquel le seigneur Nguyễn avait confié l'éducation de son fils Cảnh, héritier du trône, put nourrir l'espoir qu'il octroierait la liberté religieuse à ses sujets. Mais, en dépit de la confiance personnelle et de la gratitude manifestée ouvertement par le nouveau souverain au prélat, l'impératif de réunification du royaume et le caractère incontournable du recours à l'armature idéologique du confucianisme pour asseoir le pouvoir monarchique eurent vite fait de compromettre les chances d'obtenir la liberté d'évangélisation ${ }^{6}$.

Un incident fut sans aucun doute déterminant: le refus du prince héritier Nguyễn Phúc Cảnh de se prosterner devant l'autel des ancêtres de la lignée des Nguyễn fut imputé à la néfaste influence chrétienne. Les mandarins, imprégnés de confucianisme par conviction comme par intérêt de caste, prirent ombrage de l'action des missionnaires qui leur apparaissaient comme une classe d'éducateurs concurrents, et de surcroît porteurs d'une doctrine dangereuse pour l'ordre social et politique. D'autres faits contribuèrent à faire pencher la balance, comme l'insurrection

6. La condamnation des rites sino-vietnamiens par les papes, et en premier lieu celle du culte des ancêtres fut sans aucun doute au cœur des conflits internes et externes des catholiques du monde sinisé. $M^{g r}$ Pigneau de Béhaine ne fut pas le seul prêtre missionnaire qui prit conscience que le culte des ancêtres n'était pas un rite d'adoration et n'avait rien de religieux. II fit souvent part de son opinion à la hiérarchie, mais il ne transgressa jamais l'interdit. Ce ne fut qu'à la fin des années 1930 que le culte des ancêtres, auquel les fidèles ne renoncèrent jamais, obtint de l'Église une reconnaissance de fait; mais il fallut attendre Vatican II (1962-1965) pour que l'interdit fût officiellement aboli par Rome. 
séparatiste de Lê Văn Khôi (1833-1835) à laquelle le missionnaire Joseph Marchand fut accusé d'avoir activement participé. Le christianisme se trouva de nouveau impliqué, comme il l'avait été auparavant, et souvent malgré lui, dans les conflits internes au corps politique vietnamien.

La disparition précoce du prince Cảnh, après celle de Pigneau en 1799, scella le sort de la catholicité au Vietnam. Un autre fils de Gia Long fut investi à sa mort (1820) sous le nom de Minh Mạng; son règne (18201840) fut le temps fort de la restauration du pouvoir royal en même temps que de l'expansion territoriale de l'empire (avec annexion d'une partie du royaume du Cambodge et de portions du territoire lao). Minh Mạng affirma son pouvoir en s’inspirant du modèle chinois. Dans les Dix articles (édit de 1834), il mit l'accent sur l'éducation en prônant le respect de l'ordre et de l'orthodoxie; le respect filial, le respect dû aux aînés et à l'empereur père de tous ses sujets, furent posés comme les valeurs fondamentales.

Les Dix articles renforcèrent un premier édit de proscription qui, en 1833, avait dénoncé le christianisme comme une religion irrationnelle, perverse, porteuse d'idolâtrie et de superstitions. Et en 1838-1839, Minh Mạng lança une véritable campagne d'éradication du christianisme qui aboutit à la grande persécution de 1841 (cent trente martyrs reconnus) et conduisit la communauté catholique au bord de l'anéantissement ${ }^{7}$. La protection des chrétiens et la revendication de la liberté d'évangélisation - justifications aussi de la participation française, aux côtés des Britanniques, aux guerres de l'opium contre la Chine (1840 et 1860) - servirent de prétexte à l'agression francaise contre le Vietnam.

Une intervention navale française isolée (1845) précéda l'expédition proprement dite et la conquête de la partie méridionale du Vietnam en 1858-1872 par un corps expéditionnaire franco-espagnol (nombre de prêtres catholiques au Vietnam appartenaient à cette nationalité). Les persécutions antichrétiennes servirent de déclencheur à l'offensive militaire française qui, en retour, motiva la proscription antichrétienne sous les empereurs Thiệu Trị et Tử Đức. Ces campagnes anticatholiques furent l'occasion de constater que le christianisme avait fait des adeptes dans tous les milieux de la société, y compris au sein du mandarinat, avec la conversion secrète de mandarins de rang élevé ${ }^{8}$.

7. II faut rappeler que les persécutions conduisaient non seulement aux tueries, aux fuites, à l'exil mais également à de nombreuses apostasies.

8. Jacob Ramsay, Mandarins and Martyrs. The Church and the Nguyen Dynasty in Early Nineteenth Century Vietnam, Stanford, 2008. 
L'extension progressive de la domination française à l'ensemble de la péninsule eut pour double effet d'assurer la protection des chrétiens et de pousser au renforcement d'un sentiment antichrétien assez général: les chrétiens locaux furent positionnés - et se positionnèrent eux-mêmes souvent - comme les alliés de fait des envahisseurs; en maints endroits, la guerre de conquête fit basculer les chrétiens persécutés, enrôlés comme auxiliaires de l'armée française, dans le mauvais camp. Et si le peuple catholique ne disparut pas, sa progression numérique à tout le moins, marqua le pas.

En parallèle, le corps ecclésiastique évolua: dans un premier temps, les persécutions réduisirent les effectifs missionnaires, si bien que fut dévolu aux prêtres indigènes un rôle plus important, avec plus d'initiative et d'autonomie - conformément aux souhaits du délégué apostolique, $\mathrm{M}^{\mathrm{gr}}$ Cuénot (1802-1861). Mais l'établissement de la domination française contraria cette évolution. Débarquèrent des bataillons de missionnaires formés dans l'esprit contre-révolutionnaire et peu ouvert qui fut celui de la majeure partie du catholicisme français du xix ${ }^{\mathrm{e}}$ siècle, un esprit que ne pouvait que renforcer un statut de position dominante: une mentalité coloniale diffuse, à la fois racialiste et paternaliste, imprégna largement les missionnaires. L'ambiance des rapports de domination produisit, à l'échelon individuel, des comportements excessifs et des dérives peu ou pas catholiques. Le R.P. Léopold Cadière (1859-1955), considéré comme le fondateur de la vietnamologie, sut déplorer et dénoncer la prégnance de ce comportement colonialiste dans les rangs du clergé missionnaire.

\section{La décolonisation de la catholicité vietnamienne}

Dès lors, c'est une position fausse, en tout cas sur le plan social, que celle des catholiques vietnamiens: d'un côté, ils apparaissent comme des auxiliaires du régime colonial, voire même comme ses principaux bénéficiaires $^{9}$. De l'autre, ils sont soumis aux mêmes contraintes que leurs compatriotes, et souffrent, sont victimes d'une sujétion commune au pouvoir d’un conquérant étranger.

9. La réalité fut plus mitigée lorsque la France devint une république laïque et lorsque de nombreux coloniaux, à commencer par des administrateurs (certains francs-maçons), firent preuve d'anticléricalisme, les différends politico-religieux passant de la métropole à la colonie. Voir Luc Garcia, Quand les missionnaires rencontraient les Vietnamiens. 1920-1960, Paris, Karthala, 2008, p. 81. 
La catholicité vietnamienne se devait dès lors d'affirmer son identité; pour ce faire, il lui fallait s'affranchir de la tutelle de l'église missionnaire en même temps que de la tutelle politique de la France. C'était en quelque sorte le minimum nécessaire pour défaire l'amalgame de la religion catholique et de l'agression étrangère. Amalgame que les événements du dernier tiers du xix ${ }^{e}$ siècle avaient créé, ou en tout cas renforcé, imposant un infléchissement malheureux à une histoire qui, au départ et dans toute la complexité du contexte politique et culturel du Vietnam d'alors, ne s'était pas ouverte dans l'évidence d'une telle compromission antinationale, selon une formulation quelque peu anachronique.

La catholicité vietnamienne trouva les artisans de son émancipation dans l'élite des lettrés, des clercs et de la bourgeoisie en formation dans les deux décennies qui précédèrent la Seconde Guerre mondiale. L'évolution du monde catholique vietnamien s'entrelaça dès lors avec celle de la société globale, en même temps qu'elle fut partie prenante des transformations du catholicisme à l'échelle mondiale.

À partir du dernier tiers du xix siècle, le Vatican renforça son attention aux problèmes contemporains et sa conscience que leur prise en compte nécessaire ne pouvait rester sans implications: industrialisation et naissance du prolétariat, sécularisation des idées et libéralisation des mœurs, montée en puissance des doctrines socialistes et notamment du marxisme, etc. La guerre de 1914-1918 fut un moment décisif. La violence déchaînée par les belligérants européens de tradition chrétienne, l'onde de choc de la révolution bolchevique s'étendant sur l'Asie, entre influence de la $\mathrm{III}^{\mathrm{e}}$ Internationale et surgissement de mouvements révolutionnaires recelant en fait bien des traits nationaux, conduisirent l'Église catholique à s'interroger sur la situation du christianisme dans le monde, et à admettre la fin de certaines évidences et la nécessité de nouvelles formes d'action.

Le pape Léon XIII (1878-1903) fut le premier à s'inquiéter des liens entre les missionnaires et les régimes coloniaux; il appela à accorder plus d'attention aux clergés locaux et non-européens. La conquête des colonies et la violence inhérente à la domination exercée par un peuple sur un autre étaient fondamentalement antichrétiennes. Deux proclamations pontificales donnèrent le signal de la décolonisation de la catholicité vietnamienne: en 1919, Benoît XV déclara contraires au christianisme la collusion voire les liens organiques entre Église et gouvernement colonial; en 1926, Pie XI dénonça la discrimination raciale au sein du clergé dans les colonies et manifesta sa crainte que la fin des régimes coloniaux ne fût accompagnée de l'éviction de la religion catholique. 
En 1922, le Vatican formalisa son encouragement au développement des églises locales en Indochine, tandis qu'au sein même des missions étrangères de Paris, $\mathrm{M}^{\mathrm{gr}}$ Jean de Guébriant en 1926 et le R.P. Cadière en 1929 appelèrent à l'égalité entre prêtres missionnaires et prêtres indigènes, pour mettre fin à une discrimination raciale contraire à la doctrine chrétienne; simultanément, ils insistèrent sur la promotion du clergé indigène.

En fait, ces missionnaires donnaient un écho favorable à un mouvement qui s'affirmait localement, et visait à engager la communauté catholique vietnamienne dans la voie de l'autonomie par rapport à l'établissement ecclésiastique colonial. Pour y parvenir, l'élite catholique porta directement ses revendications à Rome. Un homme joua un rôle déterminant: ce fut le mandarin Nguyễn Hữu Bà ${ }^{10}$. En 1922, profitant du placement de l'Office de la propagation de la foi sous le contrôle direct du Vatican, ce dernier se rendit à Rome pour présenter au pape quatre revendications: des manuels de catéchisme en quốc ngử, l'uniformisation des textes d'enseignement pour les séminaires, l'établissement d'une hiérarchie ecclésiastique vietnamienne, l'instauration d'une journée de célébration des martyrs.

L'accent fut mis sur la formation du clergé: peu après le voyage de Nguyễn Hữu Bài à Rome furent créés les grands séminaires de Hanoi et Nam Dinh. Le principal périodique interne, Sacerdos Indosinensis, devint bilingue et, de manière générale, l'édition catholique connut un remarquable essor; dans les années 1920-1930, la presse catholique se développa dans les mêmes proportions que la presse laïque et d'opinion. En 1941, les publications en quốc ngử devinrent la norme ${ }^{11}$.

La revendication d'une hiérarchie ecclésiale indigène reçut une réponse un peu plus tard, dans les années 1930: trois évêques furent ordonnés entre 1933 et 1938. En 1938, dans son homélie d'investiture, $\mathrm{M}^{\mathrm{gr}} \mathrm{Ngô}$ Đình Thục, tout en rendant grâce aux missions étrangères de

\footnotetext{
10. Nguyễn Hữu Bài (1863-1935), mandarin qui occupa de très hautes fonctions sous l'empereur Khải Định puis sous Bảo Đại. II fut l'exemple même du lettré cultivant les valeurs sociomorales du confucianisme tout en étant très attaché à la foi chrétienne. II illustre la compatibilité du christianisme et du confucianisme que l'on trouve chez d'autres lettrés du XIXe siècle. Son cas, qui ne fut pas unique, éclaire l'existence d'autres lettrés et mandarins qui survécurent aux proscriptions sous Thiệu Trị et Tử Đức. Son action pour autonomiser le catholicisme vietnamien par rapport à l'Église missionnaire et lui donner une identité nationale, révèle qu'une partie de l'élite, accusée de collaboration ou de servilité, restait profondément patriote tout en adoptant une stratégie de contournement.

11. Voir Philippe Peycam, The Birth of Vietnamese Political Journalism. Saigon 1916-1930, Columbia University Press, 2012.
} 
Paris d'avoir édifié l'Église du Vietnam, martela que celle-ci était fille de Rome et ne dépendait que du Vatican ${ }^{12}$.

À la fin de la Seconde Guerre mondiale, en 1945, la nationalisation du clergé vietnamien était d’ores et déjà accomplie: il y avait 1400 prêtres, 2800 catéchistes et 5000 religieuses indigènes pour moins de 700 étrangers (330 missionnaires et 350 religieuses). Les fidèles n'en demeuraient pas moins, pour autant, une minorité au sein de la population : 1,8 million de catholiques pour 20 millions d'habitants.

\section{Des décennies 1930 et 1940 décisives}

Elles furent décisives pour que la catholicité vietnamienne prenne sa place dans la société et la culture du Vietnam.

Le peuple catholique connut de profonds changements, les laïcs évoluant de pair avec les clercs. Mais surtout, cette minorité avança à l'unisson de la société globale et ne fit que coller de plus en plus à celle-ci. La catholicité vietnamienne intégrait depuis le $\mathrm{XvII}^{\mathrm{e}}$ siècle des paysans, des pêcheurs, des soldats, des lettrés, voire des mandarins; dans la première moitié $\mathrm{du} \mathrm{xx}^{\mathrm{e}}$ siècle, elle prit aussi une forte dimension citadine; émergèrent en son sein des travailleurs manuels, des bourgeois comme des intellectuels.

Une même dialectique associa le renforcement des liens intracommunautaires (avec développement des associations cultuelles, des confréries, des mutuelles, des pèlerinages, du scoutisme) et une ouverture sur l'horizon international et les temps forts mondialement partagés: congrès eucharistiques (1928, 1931, 1935, 1937), rencontres de la jeunesse (Rome, 1939). Les catholiques furent ainsi conduits à la fois à approfondir leur foi religieuse et ses pratiques et à se sensibiliser aux questions sociales et politiques concernant les femmes, la jeunesse, le monde du travail: Nguyễn Mạnh Hà, contrôleur du travail, implanta la JOC à Haïphong; en Annam, Ngô Đình Cần fonda en 1933 un mouvement social-chrétien, inspiré de la démocratie chrétienne italienne et française; toutefois, il ne connut pas d'essor réel.

Il s'opéra une convergence avec la réflexion des non-catholiques sur la société, la nation, le confucianisme et le socialisme. Mais en même temps que les catholiques se positionnaient sur ces enjeux, ils eurent à affronter les risques et défis liés à l’influence croissante du communisme

12. Ces nominations de prélats indigènes provoquèrent des réactions hostiles de la part de certains prêtres missionnaires (refus ou interventions en haut lieu), voir Luc Garcia, op. cit. 
international porté par le Parti communiste indochinois. Au sein de la mouvance catholique elle-même, des divergences d'orientation apparurent entre une aile traditionaliste et conservatrice et une aile progressiste.

La révolution de 1945 et la proclamation d'une république indépendante (la République démocratique du Vietnam) conduisirent les catholiques à se démarquer clairement du régime colonial et à participer au grand élan patriotique: les quatre évêques catholiques firent une déclaration qui manifesta leur adhésion à l'indépendance nouvellement proclamée ${ }^{13}$.

Mais les divergences entre catholiques et non catholiques se cristallisèrent pendant la guerre d'indépendance (1945-1954) ${ }^{14}$ et surtout après la partition du pays en deux États de part et d'autre du $17^{\mathrm{e}}$ parallèle. Le régime autoritaire instauré par le clan catholique des $\mathrm{Ngô}^{15}$ fit supporter à la catholicité vietnamienne une deuxième hypothèque dont les effets se font sentir encore aujourd'hui.

En dépit de l'amalgame entre religion catholique et domination étrangère pendant la période coloniale, la catholicité vietnamienne est aujourd'hui pleinement intégrée dans la nation et dans son histoire. Les catholiques restent une minorité: selon les derniers chiffres disponibles (2010) six millions de fidèles répartis dans vingt-six diocèses ${ }^{16}, 6000$ églises, 15000 prêtres, six grands séminaires. Mais ils occupent une place et jouent un rôle à la fois visible et déterminant au plan national et international. Et la résilience de la catholicité vietnamienne démontre que les Vietnamiens, dans l'ensemble, ont parfaitement intériorisé le message

13. En septembre et novembre 1945, Mوrs Nguyễn Bá Tòng, Hồ Ngọc Cẩn, Ngô Đình Thục, Lê Hữu Từu adressèrent deux messages au pape et aux chrétiens du monde entier pour qu'ils soutiennent le Vietnam nouvellement indépendant, Archives des Missions étrangères de Paris DH 300. Le premier du 23 septembre 1945 fut publié dans Témoignage chrétien du 30 nov. 1945. II fut distribué à la porte de l'église Notre-Dame des-Champs le 25 novembre.

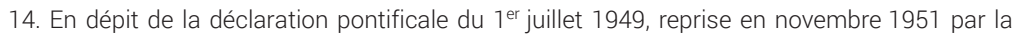
lettre pastorale des évêques vietnamiens qui interdisait aux catholiques de s'allier avec les communistes, de nombreux catholiques participèrent ou soutinrent la résistance anti-française dirigée par les communistes (voir Ngô Van Chiêu, Journal d'un combattant vietminh, Paris, 1954, Luc Garcia, op. cit.) Si pendant l'exode de 1954-1955, 600000 à 800000 chrétiens choisirent de fuir vers le Sud, 200000 à 300000 restèrent dans le Nord.

15. Ngô Đình Diệm fonda et présida la République du Vietnam de 1955 à 1963.

16. Un nouveau diocèse a été créé en 2014. 
des Évangiles. Des témoignages significatifs de la transculturalité acquise et reconnue sont apparus depuis le Đổi Mới (que l'on traduit par rénovation ou restructuration, lorsqu'en 1986, le Parti communiste adopta une nouvelle ligne politique semblable à la Perestroïka soviétique). On peut citer la restitution par les autorités d'Hô Chi Minh-ville du centre Alexandre de Rhodes à la province du Vietnam de la Société de Jésus, l'ouverture d'un séminaire pontifical sous l'égide des jésuites (à Thử Đức) et l'annonce de l'ouverture d'une université catholique privée en 2015 à Hô Chi Minh-ville. L'entrée du Vietnam dans le Đổi Mới a permis le renouveau du pèlerinage de La Vang (province de Quảng Trị) qui date du $\mathrm{xvIII}^{\mathrm{e}}$ siècle et tourne autour du culte de la Vierge Marie. Du 13 au 15 août 2014, 200000 à 300000 prêtres et fidèles venus de tout le pays y ont témoigné de la profonde et indéracinable ferveur du peuple catholique au Vietnam ${ }^{17}$.

17. Le pèlerinage avait reçu une forte impulsion sous le règne de la famille Ngô : c'est pourquoi le renouveau du pèlerinage de La Vang est un signe que l'hypothèque qui pesait sur le catholicisme à cause de la collusion perçue comme une affaire politique (entre 1955 et 1963), est aujourd'hui levée. 



\section{5}

\section{Un bouddhisme vietnamien de France?}

\section{Des hommages aux défunts comme facteur d'émergence identitaire Jérôme Gidoin}

Le premier lieu de culte bouddhique en France est édifié en 1917 à Fréjus $^{1}$, dans les limites d'un camp militaire, par les premiers migrants vietnamiens, en l'honneur de leurs camarades annamites ${ }^{2}$ morts pour la France lors de la Première Guerre mondiale. Il faut attendre l'arrivée des rapatriés indochinois en 1954, à la suite des accords de Genève, pour que le bouddhisme vietnamien commence véritablement à s'implanter ${ }^{3}$. Mais c'est à partir du milieu des années 1970, avec l'arrivée massive des Vietnamiens fuyant le régime communiste, qu'il s'installe durablement. Un grand nombre de centres bouddhiques voit alors le jour. Dès les

\footnotetext{
1. Le lieu fut officiellement reconnu par la ville de Fréjus comme espace cultuel, c'est-à-dire comme une pagode à part entière, à partir de 1971.

2. Des tirailleurs tonkinois ont également été mobilisés. S'il n'en est pas fait mention, c'est probablement en raison d'une mauvaise lecture de l'histoire vietnamienne de la part des exilés sud-vietnamiens militants, très hostiles au régime communiste assimilé aux Vietnamiens du Nord, et étrangement aux Tonkinois.

3. Certains rapatriés, par exemple, reprirent le culte bouddhique à la pagode de Fréjus, où leurs prédécesseurs avaient laissé une œuvre admirable, mais laissée à l'abandon de très nombreuses années. Une association bouddhique fut constituée le 9 novembre 1967 pour accueillir les fidèles toujours plus nombreux. La rénovation de la pagode fut achevée en 1972. Une nouvelle étape a été franchie avec l'édification d'un grand bâtiment de culte en 1978.
} 
années 1990, on recense pas moins de vingt-deux centres bouddhiques vietnamiens en France, dont neuf en région parisienne ${ }^{4}$.

On retrouve le même phénomène dans tous les grands pays d'accueil: États-Unis, Canada, Australie, et, en Europe, Suisse, Norvège, Danemark, Allemagne, Pologne.

Ce bouddhisme de la diaspora ${ }^{5}$, marqué par une forte et encore récente réalité d'exil, plus tant politique qu'économique et culturelle, s'organise en réseaux associatifs, nationaux et transnationaux; on en compte deux principaux, l’Église bouddhique du Vietnam unifiée (ÉBVU), le plus important, et la congrégation mondiale Linh Son.

Le présent article se focalisera sur l'ÉBVU, dans la mesure où il s’agit, comme nous le verrons, du courant le plus structuré et le plus représentatif de la communauté vietnamienne exilée. Cela ne signifie nullement méconnaître l'importance propre et le positionnement spécifique de la congrégation Linh Son; cette dernière compte une cinquantaine de pagodes dans le monde, dont une dizaine en France; son siège mondial se trouve au monastère de Joinville-le-Pont, en région parisienne. Son orientation religieuse est axée sur la propagation de l'enseignement du Bouddha (Dharma) au sein du courant Mahâyana. Son fondateur, le vénérable Thich Huyên Vi (1934-2005), a beaucoup fait pour la diffusion du bouddhisme en Occident, notamment en créant un établissement supérieur d'études bouddhiques en France, visant à former des moines pour assurer la poursuite cette œuvre de prosélytisme ${ }^{6}$.

\section{Un arrière-plan politique}

Ce qui, en terre d'exil, caractérise au premier chef le bouddhisme vietnamien, tel que le représente l'ÉBVU, c'est son arrière-plan politique.

Ce rapport au politique n'est pas nouveau: il est déjà présent dans le contexte colonial indochinois au début du $\mathrm{xx}^{\mathrm{e}}$ siècle. Et pour comprendre la situation du bouddhisme vietnamien en France, une brève rétrospective historique est nécessaire. À cette époque, pour les responsables des

4. Dinh Trong Hieu, "La pagode au Vietnam et dans l'immigration », Hommes et Migrations $N^{\circ} 1171 /$ décembre 1993, p. 25. Nous ne disposons pas de recensement officiel en ce qui concerne la situation actuelle, mais nous pouvons raisonnablement estimer qu'il existe une trentaine de centres bouddhiques vietnamiens en France. Mentionnons notamment l'édification de la plus grande pagode d'Europe à Évry en 2010, cf. p. 5.

5. Gidoin J., (2013a) « La pagode, lieu de mémoire d'un bouddhisme vietnamien transnational », dans la revue Carnets de Géographes, $n^{\circ} 6$ « Géographies des faits religieux », dossier coordonné par Frédéric Dejean et Lucine Endelstein, p. 2.

6. La congrégation est aujourd'hui présidée par le vénérable Thich Tinh Hanh. 
différentes organisations bouddhiques, la perspective d'un bouddhisme rénové, c'est-à-dire modernisé, tant au niveau doctrinal qu'organisationnel, apparaît comme un remède à l'altération de l'identité vietnamienne induite par la domination coloniale. On met en avant l'adéquation du bouddhisme avec le monde moderne et sa capacité émancipatrice aussi bien pour l'individu que pour la collectivité. En 1934, par exemple, l'important organe de presse bouddhiste Vien Am ("La Voix parfaite ") met en avant quatre arguments qui justifient cet engagement bouddhiste:

1) Les valeurs confucéennes traditionnelles qui déterminaient l'ancienne structure de la société sont en crise, et probablement en phase de disparition; 2) de plus, le bouddhisme peut fournir les fondations d'une identité vietnamienne adaptée à la modernisation de la société; 3) le bouddhisme est non seulement compatible avec la science moderne mais il peut lui fixer des limites morales; 4) pour pouvoir jouer ce rôle, le bouddhisme doit se séparer des rituels compliqués, des superstitions locales, afin de servir les générations futures qui auront ainsi une approche rationnelle de la connaissance ${ }^{7}$.

Parallèlement, dans les années 1930, l'école de la Terre pure ${ }^{8}$ rencontre un grand succès auprès des couches populaires, davantage par le pragmatisme de l'amidisme que préoccupé, à l'instar des moines activistes, par la rénovation de la doctrine. Quoi qu'il en soit, le soutien de la population incite les représentants des différentes associations mahayanistes à se rapprocher afin de coordonner leurs activités. Dès lors, on peut

7. Lioger R., Le bouddhisme mondialisé, Ellipses, 2004, p. 194-195. Notons que cette idée de la modernité du bouddhisme est née au Japon à la fin du XIXe siècle, cf. p. 170-172.

8. L'amidisme ou école de la Terre pure (Tịnh đo) a été introduit assez tard au Vietnam. Si dès le IXe siècle, la dévotion à Amitâbha y était connue, elle est demeurée assez discrète au cours de la longue période où le Thiên (Chan en chinois : école classique du bouddhisme chinois) domine le bouddhisme vietnamien. Unie au Thiên par le maître chinois Thao-Duong, l'école de la Terre pure a connu un succès populaire foudroyant au cours du XXe siècle, à partir des années 1930, tout en demeurant au sein du Thiên. D'abord considérée comme une pratique auxiliaire du culte du bouddha Sâkyamuni, la pratique de l'invocation du nom d'Amitâbha, en vietnamien Nam mô A Di Đà Phật (adoration au Bouddha Amitâbha), pratique facile destinée à faire renaître le fidèle en Sukhâvatî (en vietnamien Tây-phương Cực-lạc) est rapidement devenue partie intégrante de la plupart des prières. Elle représente aujourd'hui la forme de bouddhisme la plus répandue parmi les laïcs vietnamiens (et dans toute l'Asie orientale). Amitâbha est ainsi censé recevoir tous ceux qui l'ont invoqué (il suffit d'avoir simplement murmuré au cours de sa vie la formule), ce qui est un moyen particulièrement rapide d'échapper aux rouages de la métempsychose, ou plutôt, dans la conscience populaire, d'atteindre au salut et au bonheur parfait, alors que, pour parvenir au même but, le Thiên exige de ses fidèles d'austères et longues pratiques. Selon un dicton vietnamien, «le Thiên est un lion et le Tịnh đo, un lion ailé ». Voir Cornu Ph., (2006) Dictionnaire encyclopédique du bouddhisme, nouvelle édition augmentée, Paris, Seuil, p. 625, et Lafont, P.- B., (1976) «Le bouddhisme vietnamien », in Histoire des religions, encyclopédie de la Pléiade (Paris, Gallimard), t.3, p. 353-370. 
dire qu'un processus d'institutionnalisation du bouddhisme vietnamien se met en place. À partir de ce moment-là, les structures d'encadrement se rationalisent, l'ensemble des organisations bouddhistes se fédèrent et s'affilient à un réseau bouddhiste mondial, de manière à ce que le bouddhisme vietnamien étende son influence dans toutes les sphères de la société et devienne une force politique majeure. Notons qu'avant ce processus d'unification, le bouddhisme vietnamien était relativement éclaté. En 1951, un congrès organisé à Huê permet le regroupement de tous les mouvements en une organisation unique, l'Association générale des bouddhistes du Vietnam, qui s'affilie à l'Association bouddhiste mondiale $^{9}$. En 1954, avec les accords de Genève, l'unité est rompue. De 1954 à 1962, selon les chiffres donnés par l'Association générale des bouddhistes à l'époque, plus de 12000 pagodes furent construites ${ }^{10}$ dans le Sud. En 1964, l'Église bouddhique unifiée du Vietnam succède à l'Association générale des bouddhistes du Vietnam; elle réunit tous les bouddhistes du Sud, qu'ils en soient originaires ou qu'ils soient réfugiés du Nord, qu'ils soient moines ou laïcs. Les responsables de l'ÉBUV, qui sont conscients d'être devenus des acteurs incontournables soutenus par des millions de partisans ou sympathisants, veulent offrir une sorte de troisième voie qui passerait par une paix négociée et la neutralité du pays. Mais cette volonté de l'Église bouddhique va être contrecarrée par l'installation d'un régime militaire au Sud-Vietnam, voulu et soutenu par les États-Unis, en raison des enjeux géopolitiques de la guerre froide. Là, des congrégations bouddhistes concurrentes, pro-gouvernementales, vont être mises en place. Après la prise de Saigon en 1975, la religion apparaissant comme la négation même du projet révolutionnaire marxiste-léniniste, l’Église bouddhique unifiée est très vite mise sous haute surveillance, et beaucoup de moines sont persécutés. Nombre d'entre eux s'exilent. Certains avaient déjà quitté le Vietnam durant les années 1960-1970.

En exil, les moines continuent la lutte et dénoncent l'autoritarisme du régime communiste. Au Vietnam, l’Église bouddhique unifiée survit difficilement depuis qu'elle a été supplantée par l’Église bouddhique parrainée et contrôlée par le Parti; elle n’a plus de siège officiel et ses dirigeants ne sont plus reconnus par les autorités. Les moines entrent alors en dissidence et la résistance est appuyée par les congrégations de l’Église

9. Cornu, Ph., op. cit., p. 695. Notons que le bouddhisme vietnamien participe d'un phénomène de mondialisation du bouddhisme en général. Cf. Roger Liogier (2004).

10. Nguyên Thê Anh, Le Sangha bouddhiste et la société vietnamienne aujourd'hui, site internet de Vietnam infos: htp://www.vinfos.com, p. 3/12. 
bouddhique à l'étranger (Europe, États-Unis, Canada, Australie). En 1984 a lieu la plus dure campagne de répression jamais menée, à l'issue de laquelle une vingtaine de religieux sont condamnés très sévèrement pour leurs " activités visant à renverser le gouvernement du peuple ${ }^{11}$ ".

On voit ainsi qu'il existe un lien indissociable entre le sort de l’Église bouddhique unifiée au Vietnam et l'émergence du bouddhisme vietnamien en terre d'exil.

\section{L'ÉBVU en France}

Entre la fin des années 1970 et le début des années 1980, environ une trentaine d'établissements bouddhiques voient le jour en France. C'est la période la plus prolifique en termes de création de pagodes ${ }^{12}$. Et il existe autant d'associations déclarées aux préfectures, relevant des lois de 1901 (but non-lucratif) et 1905 (séparation de l'Église et de l'État) que de pagodes instituées ${ }^{13}$. Faisons remarquer par parenthèse que l'existence (minoritaire ${ }^{14}$ ) de pagodes progouvernementales affiliées à l'ambassade de la République socialiste du Vietnam ne constitue pas véritablement un facteur de scission pour la communauté bouddhiste vietnamienne: les exilés, dont la majorité est arrivée après 1975, ont de bonnes raisons d'éprouver de l'antipathie pour le régime communiste.

Dans les années 1970-1980, la congrégation de l'Église bouddhique unifiée en France est représentée par l'association bouddhique Khanh Anh de la pagode de Bagneux en région parisienne. Celle-ci a été fondée par le vénérable Thich Minh Tâm, figure charismatique du bouddhisme vietnamien en exil, président de l'ÉBVU en Europe, qui est arrivé en France en 1973 après avoir suivi des études bouddhiques au Japon, et qui n'est jamais rentré au Vietnam. La pagode de Bagneux est un espace d'expression politique autant que religieuse et culturelle. Elle édite sa revue où cohabitent motions politiques antigouvernementales et textes

11. Nguyên Thê Anh, ibid., p. 7/12.

12. Jarnot S., "Historique et organisation des bouddhismes en France », Hommes et Migrations N 1234/Novembre-décembre 2001, p. 105.

13. Tran Thi Nhung, "Le bouddhisme vietnamien en France, Tentatives de structuration », in Hommes et Migrations, $n^{\circ} 1134$ - juillet 1990, p. 28.

14. Le seul temple à l'époque dont la construction a été partiellement subventionnée par l'ambassade a reçu le sobriquet de «pagode d'ambassade » selon un jeu de mot vietnamien (chùa quán sứ " Pagode sise à l'emplacement de l'accueil aux anciennes ambassades chinoises à Hanoi », en inversant les mots, devient chùa sứ quán « pagode ambassade »), et des quolibets atteignent les religieux qui y résident, appelés bonzes d'État, su nhà nước. Cf. Dinh Trong Hieu., op. cit., p. 24, 25. 
consacrés aux activités religieuses; elle cherche aussi à fédérer au maximum en mobilisant les ressources symboliques de la culture vietnamienne.

Historiquement et en termes de fréquentation, la pagode de Bagneux est la plus importante en France ${ }^{15}$. À l'origine, ce n'était qu'un petit pavillon de banlieue; il s'est agrandi dans les années 1980 pour accueillir davantage de fidèles, de plus en plus nombreux en région parisienne. $\mathrm{Au}$ milieu des années 1990, l'association bouddhique de Bagneux a entrepris de construire une grande pagode à Évry, afin de disposer d'un haut lieu d'enseignement, de formation des moines et d'échange entre bouddhistes. Les travaux ont commencé au milieu des années 1990 et se sont terminés en 2012. Leur coût fut évalué à environ 7, 6 millions d'euros, en grande partie financés par la communauté bouddhiste affiliée à la pagode. Une grande statue du Bouddha, parée d'or, haute de quatre mètres et pesant plus de cinq tonnes, surplombe l'autel principal.

La pagode d'Évry est aujourd'hui le siège de l'ÉBVU en Europe; elle est le plus grand édifice bouddhique du continent, toutes obédiences confondues $^{16}$, et peut accueillir jusqu'à 1500 personnes, ce qui lui donne une dimension institutionnelle de premier plan, y compris à l'échelle internationale. Ce nouvel espace religieux représente à coup sûr un des hauts lieux de l'avenir du bouddhisme vietnamien en terre d'exil.

Dans ce long processus d'expansion, l'engagement des laïcs a été déterminant. Ce sont majoritairement des bénévoles qui, souvent, arrivés à l'âge de la retraite, redécouvrent le bouddhisme en France; ils s'investissent alors dans la vie associative en se livrant, parallèlement aux pratiques religieuses, à différents types d'activités qui permettent à la pagode de subvenir à ses besoins; ainsi, par exemple, ce que l'association appelle le repas social annuel, une fête qui a pour objectif de réunir un maximum de fidèles afin de collecter des dons. En bon pragmatisme, on organise une prière bouddhique collective et un hommage aux défunts dans la matinée, puis un grand repas convivial suivi d'un spectacle dans l'après-midi. Les familles et les fidèles se retrouvent autour d'une table dans un environnement vietnamien réaménagé.

15. Dans la mesure où nous avons réalisé une enquête ethnologique dans les pagodes de Bagneux et d'Evry de 2004 à 2008, nous nous appuyons ici sur une connaissance directe du terrain.

16. En 2008, en cours de construction, la pagode d’Evry a été bénie par le dalaï-lama. 


\section{L'activisme bouddhique en terre d'exil}

$\mathrm{Au}$ regard de cette rapide mise en perspective de l'implantation du bouddhisme vietnamien en France, et à la constatation de ce lien de l'histoire du bouddhisme vietnamien à la dimension politique, on pourrait penser que l'affiliation à la pagode, à côté des activités proprement religieuses et culturelles, implique inévitablement un engagement militant de la part des exilés. En fait, il faut voir que l'action des moines se distribue à deux niveaux au moins.

Le premier est effectivement politique: il est lié au sort des religieux au Vietnam et à la défense des libertés religieuses. Et l'activisme sur ce plan fait d'autant plus de sens que l'Église bouddhique unifiée est parvenue à se maintenir jusqu'à aujourd'hui au Vietnam, malgré la répression et son refus d'intégrer l'église d'État; elle est même devenue une force d'opposition qui a gardé une base populaire, en particulier au sein de la jeunesse $\mathrm{e}^{17}$. On observe aussi que le régime lâche un peu de lest parce que l'idéologie communiste est de moins en moins crédible à mesure que le pays se libéralise économiquement. Il semblerait enfin qu'une partie de la population, y compris chez les jeunes, se tourne de plus en plus vers la religion pour redécouvrir les racines spirituelles de la culture vietnamienne ${ }^{18}$. Avec une base idéologique ancrée dans la culture et un soutien populaire, l'ÉBVU dispose donc d'une marge de manœuvre dans son opposition au régime, ce qui explique aussi les tensions permanentes entre l'État et les religieux.

Le second niveau, sur lequel nous allons maintenant insister, est celui d'une action qui se donne pour axe les préoccupations réelles des exilés, qu'elles soient d'ordre religieux, culturel, identitaire, mémoriel ou encore eschatologique. Car les Vietnamiens de France, bien qu'ils restent très attachés à leur culture native, ont reconstruit leur vie ici et voient les événements passés et actuels au Vietnam avec une certaine distance. Et c'est encore plus vrai, évidemment, pour leurs enfants et petits-enfants. La plupart des fidèles aujourd'hui ne s'intéressent plus aux questions politiques. Les moines en ont conscience et savent que l'avenir français du bouddhisme vietnamien ne peut être envisagé qu’à partir du vécu de cette population, désormais profondément implantée dans le pays. Le bouddhisme vietnamien en France se dessine en perspective comme un bouddhisme vietnamien de France, se projetant dans l'avenir à partir

17. Nguyên Thê Anh, op. cit., p. 8/12.

18. Nguyên Thê Anh, op. cit., p. 10/12. 
de la situation française. Citons ici le vénérable Thich Minh Tâm, interviewé dans l'émission de télévision "Sagesses bouddhistes » en 2007, qui aborde deux sujets fondamentaux liés au contexte français, d'une part, l'avenir de la jeunesse bouddhiste franco-vietnamienne et, d'autre part, le rapport qu'entretient le bouddhisme vietnamien avec les autres traditions bouddhistes de France:

L'objectif de la pagode d'Évry, aujourd'hui, est de former des moines et des nonnes pour continuer à développer le bouddhisme vietnamien en France. [...] La pagode favorise les activités religieuses et sociales pour les jeunes Vietnamiens. Ces enfants, nés en France, devraient pouvoir garder leurs traditions culturelles et religieuses. [...] Il devient difficile pour les enfants élevés en France de comprendre la langue, la culture vietnamienne et la religion bouddhiste. [...] L'adaptation se fait petit à petit et se fera tout de même, d'une autre façon.

Oui, pour nous c'est un plaisir d'assembler toutes les traditions bouddhistes à la pagode d'Évry, parce qu'elles ont un seul but: le Bouddha et son enseignement, c'est-à-dire résoudre les douleurs de la personne sur la terre ${ }^{19}$.

Cette perspective ouverte par le contexte post-migratoire est essentielle puisque ce sont les laïcs sympathisants qui, par leurs dons, permettent à la pagode de réaliser ses projets. Rappelons que la grande pagode d’Évry a été principalement financée avec les dons des fidèles. C'est en grande partie grâce à ces dons que le bouddhisme vietnamien a pu se développer. Et ce qui est intéressant, c'est qu'ils proviennent essentiellement des offrandes en espèces effectuées par les fidèles qui ont délégué leur culte des ancêtres aux moines.

\section{Un essor via la prise en charge des hommages aux défunts}

À l'issue de notre enquête, nous pouvons dire en effet que le bouddhisme vietnamien s'est développé par le biais d'une politique de gestion de l'identité culturelle principalement axée sur l'intégration des pratiques d'hommage aux ancêtres. Chaque dimanche matin, la pagode organise des cérémonies consacrées aux défunts qui rassemblent en général une centaine de personnes venues en famille. Dans la salle de prière, deux

19. Propos de Thich Minh Tâm, invité de l'émission de télévision "Sagesses bouddhistes » sur France 2, le 11 novembre 2007, cf. site internet: http://www.bouddhisme-France.org/voixbouddhistes/detail-des-emissions/071111.htm 
grands autels réservés aux ancêtres occupent une place importante et cohabitent avec l'autel du Bouddha.

$\mathrm{Au}$ niveau d'ampleur qu'il a pris en France, ce phénomène est assez inédit. Avant l'exil, disons dans le centre et le sud du Vietnam d'avant 1975, d'où sont originaires la plupart des exilés, le culte des ancêtres et le bouddhisme constituaient deux cultes bien distincts. Le culte des ancêtres $^{20}$ se pratiquait dans l'espace domestique. Les familles avaient recours au service des moines pour qu'ils effectuent quelques prières au moment des funérailles, mais les hommages aux défunts, en aucun cas, ne se pratiquaient de façon définitive et régulière à la pagode. Par contraste, en France, les moines vietnamiens sont en quelque sorte parvenus à obtenir le monopole des pratiques d'hommage aux défunts.

Avec le temps, après quatre décennies très souvent, les générations se succédant, les rapports à la culture vietnamienne à la famille ainsi qu'à la mort ont connu des mutations. C'est pourquoi nous pouvons raisonnablement faire l'hypothèse que les familles sont à la recherche de nouvelles modalités pour continuer d'honorer leurs défunts et transmettre certaines valeurs aux plus jeunes. Il est vraisemblable que le ritualisme familial traditionnel s'est progressivement déstructuré au contact de la société d'accueil (c'est un fait empirique que nous avons fréquemment constaté dans l'espace domestique). Ce phénomène a des conséquences sur la relation qu'entretiennent les Vietnamiens avec leurs défunts. Or, tous ceux qui s'intéressent à la culture vietnamienne le savent, l'échange symbolique avec les défunts, en termes axiologiques, joue un rôle essentiel dans la vie des Vietnamiens. Pour compenser les effets de l'acculturation, la majorité d'entre eux dispose d'une ressource culturelle qu'elle peut mobiliser, à savoir le bouddhisme, quitte à ce qu'il soit réinterprété de multiples façons. Sur le plan pratique, le fait de transférer le culte des ancêtres à la pagode simplifie la vie des familles, car elles n'ont plus besoin d'entretenir le culte à leur domicile, dans la mesure où les moines prennent le relais en récitant des prières et en effectuant fréquemment des offrandes à leur place.

Les moines, de leur côté, gagnent de nouveaux adeptes puisque pour pouvoir bénéficier des rites adressés aux défunts, une famille doit adhérer à l'association bouddhique de la pagode et faire assez régulièrement des offrandes en espèces. Rappelons qu'en France, les pagodes dépendent presque entièrement des dons des fidèles, sans lesquels elles

20. Le culte des ancêtres est un aspect du culte des génies que l'on retrouve dans toute l'Asie du Sud-Est. II a été rationalisé par le confucianisme comme d'autres aspects des cultures sinisées, notamment au XVE siècle, période d'occupation chinoise. 
ne pourraient tout simplement pas exister. Les moines disposent par conséquent d'un moyen efficace pour fidéliser les laïcs.

Au-delà de l'aspect pratique, la pagode offre la possibilité de réaménager un environnement social vietnamien mobilisable périodiquement, et plus particulièrement pour les anciennes générations, elle apporte une sécurité existentielle, à la fois identitaire et eschatologique ${ }^{21}$. L'affiliation au bouddhisme permet également de recomposer une éthique sociale qui peut répondre à de nombreuses attentes. Pour les premières générations, le bouddhisme en France intègre les représentations du patrimoine éthique et culturel vietnamien, et pour les nouvelles générations, il est porteur de valeurs universelles souvent perçues en adéquation avec le monde contemporain.

\section{Assimilation de l'identité bouddhique à l'identité culturelle}

L'intégration des hommages aux défunts au bouddhisme, qui s'est faite avec la bénédiction des moines, plutôt tolérants à l'égard des pratiques religieuses populaires, résulte d'une stratégie de conversion consistant à assimiler l'identité bouddhique à l'identité culturelle. Pour illustrer ce phénomène, nous ferons référence à un événement qui s’est déroulé à la pagode de Bagneux durant l'été 2005. Une délégation de la ville de Bagneux était venue inspecter la pagode de fond en comble et avait réalisé un compte rendu, à la suite de quoi, le jour même, le maire avait signé un arrêté interdisant l'une des principales activités bénévoles, à savoir la préparation des mets végétariens destinés aux fidèles le dimanche matin. Les représentants de la municipalité avaient interprété et caractérisé les activités des femmes de l'association comme un service de restauration lucratif, incompatible donc avec le statut associatif ${ }^{22}$. Or, l'expression d'exercice de la restauration, motivant cette mesure, ne correspondait guère à l'activité et au ressenti de bénévoles visant à récolter des dons afin de subvenir aux besoins de la pagode ${ }^{23}$. En réponse à l'arrêté du maire, le vénérable rédigea une plaidoirie où il présenta officiellement

21. Gidoin J., (2013) «Affiliation identitaire et affiliation eschatologique comme richesses primordiales chez les Vietnamiens en France », in Archéologie et Ethnologie, $n^{\circ} 8$ « Richesses et société », sous la direction de Catherine Baroin et Cécile Michel, colloques de la Maison René-Ginouvès.

22. Cette réaction de légalisme face à une situation mal comprise faisait suite à une plainte d'une personne du voisinage à l'intention quelque peu malveillante.

23. Il est vrai qu'au Vietnam, il est impensable de vendre de la nourriture dans une pagode, c'est un phénomène propre au contexte post-migratoire. 
les principes qui fondent selon lui l'identité du bouddhisme vietnamien en France. Dans sa lettre, il écrit que l'une des principales finalités de l'association bouddhiste est de préserver la culture bouddhique pour les Vietnamiens exilés. Il est également question de "préserver les us et coutumes (sous-entendu le culte des ancêtres, “institution” en quelque sorte par laquelle il les résume) de la religion bouddhique ". La culture bouddhique assimile clairement ici le culte des ancêtres.

En porte-parole des exilés militant pour la défense de leur culture et de leurs pratiques religieuses ancestrales, le vénérable dramatise l'événement, allant même jusqu'à imaginer une " dissolution " de la pagode. Il laisse entendre que la cessation des activités dites de restauration menace la pérennité de la pratique du culte des ancêtres, et avec elle, l'identité culturelle des Vietnamiens. L'arrêté du maire est interprété comme un acte « assimilationniste », voire " ethnocidaire » :

Les conséquences entraînées par votre arrêté nous imposent de couper immédiatement tout lien avec nos racines, nos traditions et nos coutumes, que nous avons préservées pendant des milliers d'années au prix de nombreux sacrifices, et transmis de génération en génération.

Pour donner du poids à son propos, le vénérable intègre les intérêts singuliers de l'association bouddhique de Bagneux à la dimension plus globale de la communauté bouddhique asiatique en France (500000 personnes environ selon lui, mais il n'existe pas en réalité de chiffres fiables ${ }^{24}$ ) en brandissant un " Nous les bouddhistes ". D’autre part, sûr de sa légitimité, en particulier parce que la communauté vietnamienne aurait la réputation d'être bien intégrée en France, avec cette image pour le moins stéréotypée du Vietnamien discret et travailleur, sans oublier l'histoire qui lie intimement les deux peuples à travers le passé colonial, il n’hésite pas à s'en référer aux instances supérieures de l'État pour qu'elles puissent juger « si la communauté vietnamienne et plus particulièrement la communauté bouddhique ont été par le passé, sont aujourd'hui ou seront à l'avenir sources de préjudice ou de bienfait envers la France ».

Ces données descriptives nous apportent des éléments tout à fait significatifs pour comprendre les ressorts de l'assimilation de l'identité bouddhiste à l'identité culturelle. À vrai dire, le phénomène est aussi ancien que l'implantation des premières pagodes vietnamiennes en France. Ce qui est inédit avec cet épisode malencontreux, c'est que l'association Khanh Anh, et à travers elle le bouddhisme vietnamien de France, puisqu'elle

24. Obadia L., (1999) Bouddhisme et Occident. La diffusion du bouddhisme tibétain en France, Paris, L'Harmattan («Religion et sciences humaines »), p. 167-170. 
demeure la plus organisée, après presque quarante ans d'existence, se présente légitimement comme l'organisation représentative de l'identité culturelle vietnamienne et la plus à même de fédérer les personnes originaires du Vietnam. Le vénérable lui-même, une des plus hautes autorités du bouddhisme vietnamien en Europe, se revendique comme le gardien des us et coutumes des Vietnamiens et normalise l'hommage aux ancêtres assimilé à une pratique de la culture bouddhiste. L'implication du vénérable, et surtout ce qu'il représente, c'est-à-dire non seulement le bouddhisme vietnamien en France et en Europe, mais également asiatique et international ("par extension » comme il l'écrit), crédibilise le bouddhisme en tant qu'institution efficace pour défendre les intérêts des Vietnamiens en situation de crise $^{25}$.

Le vénérable, à travers sa lettre, et les bénévoles de l'association Khanh Anh, à travers leur mobilisation auprès des sympathisants - l'affaire eut un certain retentissement - , activent une frontière identitaire et la question de l'ethnicité, au sens où l'entend le sociologue F. Barth ${ }^{26}$, comme " usage stratégique et sélectif de la culture visant à maintenir ou à remettre en cause des frontières identitaires ". C'est une façon pour eux d'asseoir l'influence du bouddhisme vietnamien en faisant preuve de pragmatisme et d'opportunisme. Lorsque le vénérable parle d'offrandes aux ancêtres, par opposition aux activités de restauration qu'a constatées l'administration, et qu'il fait état d'une pratique caractéristique de la culture vietnamienne (judicieusement associée au bouddhisme), il opère d'autorité, en quelque sorte, une requalification de la chose par introduction dans les mots d'une dimension spirituelle. Le vénérable joue délibérément sur la sémantique, en sachant que l'image du bouddhisme est perçue de manière positive en Occident. En stratège, il envisage aussi l'avenir du bouddhisme, car sans ces dons, la pagode n'est pas viable et l'expansion de cette religion inimaginable.

\section{La pagode, un symbole de la vietnamité en France}

En novembre 2008, le même jour, deux monuments ont été inaugurés à la grande pagode d’Évry: un stûpa de neuf étages accueillant les cendres

25. Faisons remarquer que le bouddhisme est probablement plus apte que le confucianisme à apporter un réconfort moral aux personnes lorsque, dans des situations de crise, les repères vacillent.

26. Barth F., (2008) «Les groupes ethniques et leurs frontières», in Poutignat Ph., et StreiffFénart J., Théories de l'ethnicité, PUF « Quadrige », p. 205-251. 
des défunts et un mémorial en hommage aux boat people ainsi qu'aux soldats de l'armée sud-vietnamienne. Les deux monuments étaient clairement associés d'un point de vue symbolique. Les familles affiliées à l'association bouddhique peuvent acheter une concession et y déposer les cendres de leur(s) défunt(s). Le stûpa, détourné de sa fonction originelle, s'apparente à une sorte de tombeau collectif bouddhiste pouvant accueillir près de quatre mille urnes funéraires. Et il est remarquable d'observer que de plus en plus de fidèles envisagent désormais leur destinée postmortem en France selon cette nouvelle option.

La pagode devient ainsi un lieu de mémoire, au sens où l'entend Pierre Nora ${ }^{27}$. Dans tous les pays de la diaspora, les mémoriaux pour les boat people sont des lieux de mémoire fédérateurs. Le mémorial fait référence aux ancêtres bâtisseurs, ce qui signifie que par-delà les déchirures engendrées par les épisodes tragiques de l'histoire vietnamienne, il existe une continuité qu'incarnerait aujourd'hui la pagode en terre d'exil. Faisons remarquer qu'en passant de l'inauguration du stûpa à celle du mémorial, nous passons d'un hommage aux ancêtres privés, c'est-à-dire familiaux, à un hommage aux ancêtres publics, l'association des deux ayant à l'évidence une fonction idéologique porteuse d'enjeux mémoriaux et identitaires.

Étant donné que la pagode d’Évry assume aujourd'hui une dimension internationale, l'édification d'un tel stûpa consacré aux défunts a indéniablement une grande portée symbolique. On peut concevoir l'idée d'un processus de structuration, en terre d'exil, d'une communauté vietnamienne à partir du bouddhisme via la prise en charge des hommages aux défunts ${ }^{28}$. Le stûpa et le mémorial objectivent au sein du plus grand lieu de culte bouddhiste d'Europe la relation indissociable entre la pagode et la mémoire des exilés. À travers la perpétuation d'une pratique du culte des défunts réaménagée, le lien entre la pagode et les fidèles est en quelque sorte consacré.

$$
* * *
$$

Nous avons cherché à montrer que le bouddhisme vietnamien s'est développé en France en intégrant les hommages aux défunts, et plus globalement, via la prise en charge de la mémoire collective des exilés.

27. Nora P., (1984), «Entre mémoire et histoire : la problématique des lieux », in P. Nora (dir.), Les lieux de mémoire, t. 1 : La République, Paris, Gallimard, p. 1-42.

28. Gidoin J., (2013a), op. cit. 
Alors qu'elle a pour cause première sa commodité, la pratique des hommages aux défunts à la pagode tend à devenir une preuve d'adhésion au bouddhisme. Cette façon chez les laïcs de normaliser leur affiliation au bouddhisme est la conséquence assez logique d'une normalisation qui s'est opérée en amont à travers la politique de gestion de l'identité conduite par l'association bouddhique. L'édification du stûpa pour les ancêtres à Évry résulte d'une relation de plus en plus resserrée entre une institution bouddhique très structurée, misant sur la carte culturelle pour s'accroître, et des fidèles vraisemblablement de plus en plus bouddhistes, dans la mesure où la pagode s'efforce de polariser les ressources symboliques de la vietnamité.

Évoquons pour terminer la situation assez paradoxale du bouddhisme vietnamien en France. On relève en effet une contradiction entre sa dimension ethnique et son message universel. Le bouddhisme vietnamien serait la forme de bouddhisme la plus répandue en France aujourd'hui; ses fidèles représenteraient environ $60 \%$ des bouddhistes de France. Mais, en raison de son communautarisme, ce bouddhisme reste aussi le plus méconnu. Notons que le bouddhisme vietnamien de France est affilié à une église bouddhique transnationale qui conserve son assise symbolique dans le pays d'origine, le quatrième patriarche de l'Église bouddhique unifiée, Thich Huyên Quang, demeurant au Vietnam ${ }^{29}$. Nous avons donc affaire à une situation un peu étonnante, d'autant plus qu'avec l'essor du bouddhisme de France, le bouddhisme vietnamien a de toute évidence son rôle à jouer, notamment au sein d'une institution comme l'UBF (Union des bouddhistes de France). La contradiction est en fait liée à la dimension transnationale du bouddhisme vietnamien en exil, avec ses tensions entre le local et le global, entre des intérêts nationaux et internationaux dont dépend son expansion.

29. Thich Huyên Quang est mort le 5 juillet 2008. Thich Quang Dô lui a succédé. 
TROISIÈME PARTIE

\section{CONSTRUCTION DES SAVOIRS}





\section{6}

\section{L'économie hybride du post-Champa}

\section{Le commerce plaine-montagne et les " marchés des sources " (nguồn) à l'époque des seigneurs Nguyễn (XVI $I^{e}-\mathrm{XVIII}{ }^{e}$ siècles) Andrew Hardy}

Un élément notable dans la formation de l'identité particulière du Vietnam, entre intégration culturelle et diversité, est à rechercher du côté de la période post-Champa et de ses marchés des sources.

La période durant laquelle les seigneurs Nguyễn gouvernent - du $\mathrm{XVI}^{\mathrm{e}}$ au $\mathrm{XVIII}^{\mathrm{e}}$ siècle - marque un tournant dans le processus de longue durée de la transition économique dans le centre-Vietnam. Avant le $\mathrm{xvI}^{\mathrm{e}}$ siècle, le modèle économique dominant est celui du Champa. Il est basé sur un système mixte reposant sur les échanges maritimes, les échanges plaine-montagne et l'agriculture. Après le $\operatorname{xvIII}^{\mathrm{e}}$ siècle, les échanges commerciaux n'occupent plus qu'une position marginale dans l'économie de la région, qui est désormais devenue largement dépendante de l'agriculture.

Ces deux modèles peuvent être distingués par leur orientation géographique respective. Dans celui du Champa, de grandes richesses sont créées par la circulation de biens de haute valeur marchande entre les hautes terres et la mer, par l'intermédiaire de ports installés dans la plaine côtière, servant de pôles d'échange et de comptoirs commerciaux 
pour des produits provenant, pour la plupart d'entre eux, des régions montagnardes ${ }^{1}$. Ce modèle est orienté est-ouest.

Le modèle vietnamien, au contraire, est basé sur la riziculture; la production est organisée à la fois par les colons-migrants venus du Nord et par les indigènes; la majorité des biens proviennent de la plaine côtière elle-même. Ce modèle est donc orienté nord-sud. Les orientations géographiques de ces deux modèles sont représentées par la figure 1.

$\mathrm{Du} \mathrm{XVI}^{\mathrm{e}}$ au $\mathrm{xvIII}^{\mathrm{e}}$ siècle, les deux modèles coexistent. D’un côté, une expansion agricole le long de la plaine côtière — du Quảng Nam jusqu'au Gia Định — due à l'installation et à l'intégration des migrants originaires du Nord au sein des populations locales, se traduit par l'augmentation de la surface des terres mises en culture, et plus particulièrement en riziculture. De l'autre, les échanges maritimes amont-aval continuent de prospérer à travers Hội An et, dans une moindre mesure, à travers les autres ports situés le long de la côte.

Durant cette période, la région centre du Vietnam présente donc une économie qu'on peut qualifier d'hybride. Tout est dès lors affaire de point de vue. Cette économie peut être considérée sous l'angle du modèle émergent, le modèle vietnamien. C'est ainsi que les historiens ont étudié cet ensemble de transformations historiques, sous le nom de nam tiến ou " marche vers le Sud ${ }^{2}$ ". Si, récemment, le terme est passé de mode, c'est probablement parce qu'il ne permet pas de rendre suffisamment compte de la force et des effets d'intégration des contacts culturels tels qu'ils se nouent entre les différents acteurs mis en rapport par le processus

1. En cela, le modèle économique du Champa comporte des similarités avec d'autres modèles d'Asie du Sud-Est, notamment celui du monde malais. Voir Geoffrey Benjamin, « On Being Tribal in the Malay World », dans Geoffrey Benjamin \& Cynthia Chou, Tribal Communities in the Malay World, Historical, Cultural and Social Perspectives, Singapore \& Leiden, ISEAS \& IIAS, 2003, p. 10 [7-76]. Voir aussi Kathleen D. Morrison \& Laura L. Junker, Forager-Traders in South and Southeast Asia, Long-Term Histories, Cambridge University Press, 2002. L'importance des relations plainemontagne au Champa et dans le centre-Vietnam est également mise en avant par Jacques Dournes, "Recherches sur le haut Champa », France-Asie, 24 février 1970: p. 143-162, et par Oscar Salemink, «A view from the mountains: A critical history of lowlander-highlander relations in Vietnam », dans Upland transformations in Vietnam, Thomas Sikor, Nghiem Phuong Tuyen, Jennifer Sowerwine \& Jeff Romm (eds), Singapore, NUS Press, 2011, p. 27-50.

2. Les recherches sur l'expansion vietnamienne vers le Sud ont été regroupées sous le nom de nam tiến, terme utilisé notamment par les historiens du Sud-Vietnam, entre 1955 et 1975. Voir le numéro spécial de Sử Địa [« Histoire et géographie »] dans " Nam tiến của dân tộc Việt Nam » " La marche vers le Sud de la nation vietnamienne »], 19-20, 1970; et le livre de Phan Khoang, Việt sử: xứ Đàng Trong, $1558-1777$ (cuộc Nam-tiến của dân tộc Việt-Nam) [« L'histoire vietnamienne : le territoire de Đàng Trong, 1558-1777 (la marche vers le Sud de la nation vietnamienne) »], Saigon, Khai Trí, 1970 [691 p.]. 
Figure 1. Orientations économiques du Champa, du Vietnam et du territoire des seigneurs Nguyễn pendant la période de transition (XVI'-XVIII siècles)

Orientation économique vietnamienne (nord-sud)

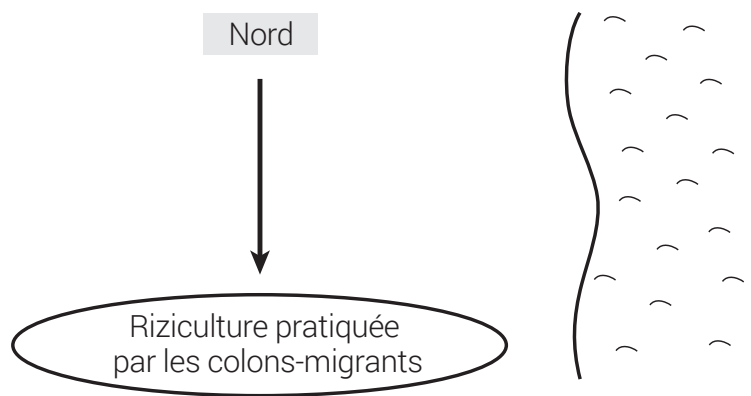

Orientation économique du Champa

(est-ouest)

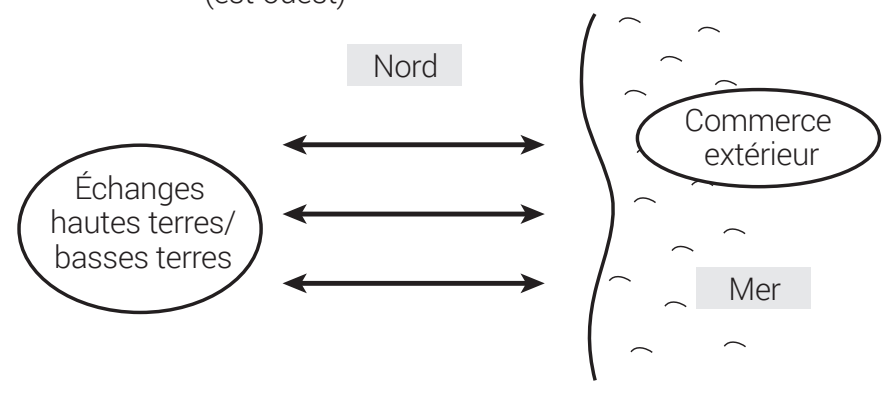

Orientation économique des seigneurs Nguyễn

(hybride)

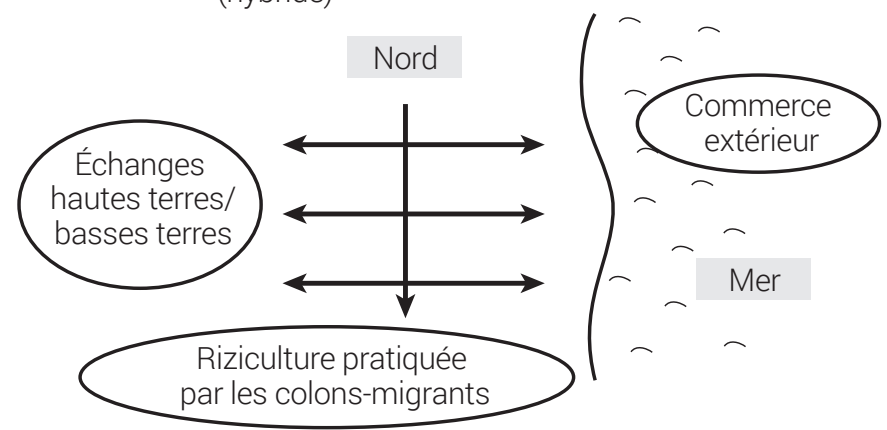


de l'expansion vietnamienne, à l'intérieur des territoires des royaumes champa et khmer ${ }^{3}$.

À l'inverse, prendre la mesure de la persistance de caractéristiques du système économique champa après l'effondrement politique du royaume au $\mathrm{xv}^{\mathrm{e}}$ siècle ne suffit pas, si cela conduit à ne cibler que l'obstination du même à se reproduire face à l'autre qui finit inexorablement par le supplanter...

Il nous paraît plus approprié et fondé de reconnaître à cette hybridité les caractéristiques d'une véritable dynamique - de transition, entre deux périodes historiques - et la cohérence, la densité d'un modèle, qu'on nommera ainsi modèle post-Champa.

Pour contribuer à l'élucidation de ce modèle et du jeu afférent de contacts culturels, nous nous concentrerons sur le principal point de contact et d'échange, social et économique, entre les différentes populations des hautes terres et des basses terres. Ce point de contact est un type spécifique de marché, connu en vietnamien sous le nom de nguồn ou đầu nguồn, ce qui qui signifie littéralement " source ». L'expression se réfère à la fois à l'élément aquatique (source d'eau ou cours d'eau alimentant une rivière, un fleuve), et à la dimension économique (la source comme lieu d'approvisionnement en biens et en marchandises). La traduction proposée: marché des sources, rend compte de cette polysémie.

L'étude de ces lieux permettra à la fois d'appréhender un fonctionnement - d'éclairer concrètement ce caractère hybride de la région durant la période post-Champa - et de mieux cibler les raisons pour lesquelles se clôt cette période, avec la crise du XviII ${ }^{\mathrm{e}}$ siècle $^{4}$.

\section{Le modèle économique post-Champa}

Ces deux modèles, vietnamien et post-Champa, ne font pas que présenter des orientations géographiques différentes (nord-sud et est-ouest).

3. Pour une critique du concept de marche vers le Sud, voir Keith W. Taylor, "Surface Orientations in Vietnam: Beyond Histories of Nation and Region », Journal of Asian Studies 57/4, 1998, p. 949-978.

4. Cet article est le développement d'idées introduites dans trois précédentes publications: " 'Nguồn'trong kinh tế hàng hóa ở Đàng Trong » ("La "source" dans l'économie commerciale à Đàng Trong »), dans Chúa Nguyễn và Vương Triều Nguyễn trong lịch sử Việt Nam từ thế kỷ XVI đến thế kỷ XIX (" Les seigneurs Nguyễn et la cour de Nguyễn dans I'histoire du Vietnam du XVIe siècle au XIXe siècle »), Hanoi, Nxb Thế giới, 2008, p. 55-65; « Núi và biển trong lịch sử kinh tế Chămpa và Việt Nam » ("La montagne et la mer dans l'histoire économique du Champa et du Vietnam »), dans Văn hóa biển miền Trung và văn hóa biển Tây Nam Bộ (" La culture maritime de la région centrale et la culture maritime du Sud-Ouest »), Hanoi, Nxb Từ điển Bạch hoa, 2008, p. 88-102; « Eaglewood and the Economic History of Champa and Central Vietnam », in Andrew Hardy, Mauro Cucarzi \& Patrizia Zolese (éds), Champa and the Archaeology of My Son (Vietnam), Singapore, NUS Press, 2008, p. 107-126. 
S'ajoutent deux autres contrastes importants: le terrain qu'ils recouvrent et les groupes ethniques qu'ils comprennent.

Schématiquement parlant, le modèle vietnamien est géographiquement et ethniquement homogène. Il s'implante sur des terrains propices à la pratique de la riziculture inondée et, de ce fait, tend à intégrer les autres groupes ethniques dans son système social et économique, qui est donc assimilateur et unificateur. Au contraire, le modèle du Champa opère sur un territoire étendu et d'une grande diversité topographique, comprenant aussi bien des forêts, des montagnes que des plaines côtières, englobant ports et îles et s'étendant jusqu'en haute mer; il y a, sur ce territoire hétérogène, mise en réseau d'une grande variété de groupes ethniques, mais pas, pour autant, unification de ces derniers: socialement et économiquement, ces groupes continuent de présenter des modèles d'organisation très divers.

Il y a donc contradiction entre le modèle du Champa, qui promeut la circulation et favorise la diversité et les caractéristiques distinctives du modèle vietnamien, qui sont l'uniformité et la centralisation et qui tendent à amoindrir à la fois la circulation et les différences.

Cette contradiction ne s'est pas résolue à la hâte. Il y a survivance au niveau local - durant les siècles suivant le démantèlement, à partir de 1471, du Champa en tant que royaume unifié - de plusieurs pratiques économiques, culturelles et politiques et donc, comme nous l'avons dit, cohérence d'un modèle intermédiaire post-Champa.

Cette période est, probablement et précisément à cause de sa nature transitoire, particulièrement pauvre sous le rapport des sources disponibles: quelques textes, datant $d u x{ }^{e}$ et $d u$ xvII ${ }^{e}$ siècle, ne délivrent que de très maigres informations; le XviII ${ }^{\mathrm{e}}$ siècle est à peine mieux documenté. Cependant, malgré cette indigence, il est clairement attesté que l'une des plus importantes survivances du Champa sous la dynastie des Nguyên est le système d'échange amont-aval.

Dans la mesure où il est peu probable que les pratiques commerciales du Champa aient survécu telles quelles, sans être soumises à aucun processus de changement, nous sommes enclins (sous bénéfice des arguments et preuves restant à réunir) à définir le "marché des sources ", objet de la présente étude, comme une innovation caractéristique de cette période post-Champa; vont de pair apparition de ce type de place et conservation des caractéristiques principales du modèle du Champa, à savoir son orientation géographique et la diversité des territoires et des groupes ethniques.

Comment ce système post-Champa fonctionne-t-il à travers les différents contextes géographiques et ethniques que son orientation 
amont-aval nécessite? Nous pouvons synthétiser ses caractéristiques en mettant en avant deux éléments principaux:

1. Le premier est le système de places d'échanges, incluant:

- Les ports côtiers, le plus important étant Hội $\mathrm{An}^{5}$ (durant la période du Champa, les îles situées le long des côtes - Cu Lào Chàm, Cu Lào Ré, etc. - ont également été utilisées comme des points de transbordement. Cette pratique disparaît durant la période post-Champa. Dans l'actuelle province de Quảng Nam, le mouvement s'accompagne de l'émergence de la ville de Đà Nẵng, point de débarquement pour les visiteurs étrangers);

- Les marchés des sources, points de collecte et de taxation des biens circulant entre les hautes terres et les plaines. Ces bourgs-marchés sont localisés le long des fleuves principaux, à l'extrémité de la plaine côtière;

- Des marchés subsidiaires, liés aux marchés des sources. Ce sont des places d'approvisionnement en biens divers achetés auprès des populations montagnardes, localisés plus en altitude sur les hautes terres, ou des lieux situés entre les marchés des sources et les ports, servant de point de collecte pour les marchandises qui circulent entre les hautes terres et les plaines;

- De multiples lieux situés dans les montagnes où les populations échangent des biens au gré de négociations informelles - dans les villages ou dans les sentiers forestiers plutôt que dans les marchés réguliers; les transactions reposent en grande partie sur la médiation des anciens du village.

2. Les places d'échanges sont liées entre elles par une série de relais d'échanges, gérés, en fonction de leur localisation, par des groupes divers. La figure 2 représente une section théorique de ce système d'échanges à travers les hauts plateaux de Tây Nguyên. Des acteurs spécifiques opèrent dans des régions géographiques distinctes: les Chinois dans les zones maritimes et les basses terres, les Việt dans les zones de basses terres et

\footnotetext{
5. Sur le commerce maritime pratiqué à Hội An, voir les chapitres 2 et 3 de Li Tana, Nguyen Cochinchina, Southern Vietnam in the Seventeenth and Eighteenth Centuries, Ithaca, New York, Cornell, 1998 [194 p.]; Chingho A. Chen, "Historical Notes on Hôi-An (Faifo) », in Eclipsed Entrepots of the Western Pacific, John E. Wills Jr. (ed.), Aldershot, Ashgate, 2002, p. 285-361. Sur la coexistence des populations cham et việt dans la région de Hội An durant la période postChampa, voir Charles Wheeler, "One Region, Two Histories: Cham Precedents in the History of the Hoi An », in Vietnam, Borderless Histories, Nhung Tuyet Tran \& Anthony Reid (eds), Madison, University of Wisconsin Press, 2006, p. 157-193.
} 
celles de moyennes altitudes, de multiples et différents groupes montagnards dans des zones montagneuses, et ainsi de suite. Chaque groupe circule entre les différentes places d'échanges, dans une seule section de la route commerciale. Aucun groupe n'opère seul à travers tout le système, dont l'hétérogénéité ethnique est ainsi assurée.

Figure 2. Le système des relais dans le système commercial est-ouest durant la période des seigneurs Nguyễn (XVI-XVIII ${ }^{\mathrm{e}}$ siècles)

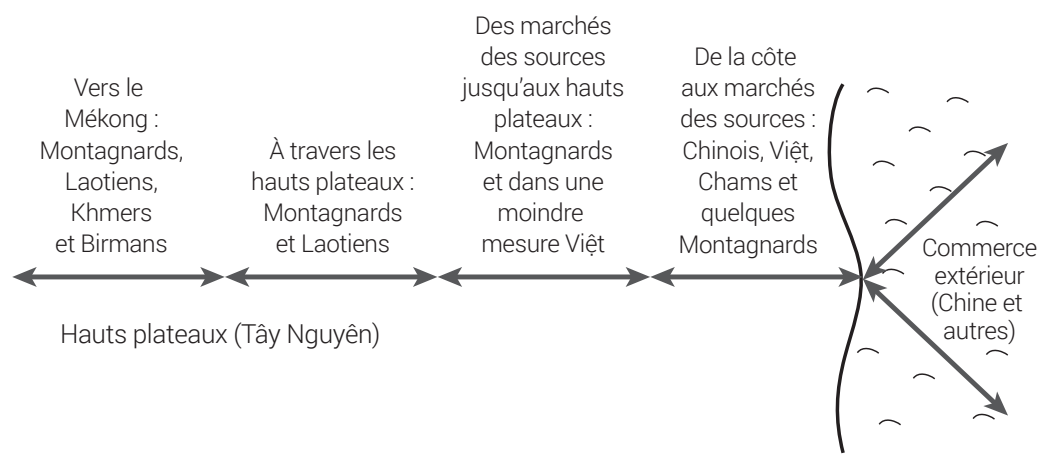

L'importance des marchés des sources repose sur leur position de pivot au sein de l'ensemble des relais d'échange. Le bourg qui se développe autour d'un tel marché est habité par des agents chinois appartenant aux grandes maisons de commerce des ports côtiers, ainsi que par des Việt travaillant pour ces agents, comme à leur propre compte. Le marché est aussi régulièrement fréquenté par des populations montagnardes. La comparaison de plusieurs documents - la compilation de documents portant sur le Sud (Phủ biên tạp lục) de Lê Quý Đôn (xvIII siècle), les archives officielles de la dynastie des Nguyễn (XIX ${ }^{\mathrm{e}}$ siècle), les récits écrits par les premiers voyageurs français et les résultats des terrains ethnographiques - nous permettent de nous faire une idée du fonctionnement de ce système.

\section{Les places d'échanges}

Un ouvrage du missionnaire Pierre Dourisboure, datant du XIX siècle, nous offre une bonne vision des conditions de voyages à travers les montagnes vietnamiennes du centre et nous permet également de mesurer la complexité des contacts culturels amont-aval. Publié en 1873, ce texte retrace les étapes suivies par les fondateurs de la mission de Kon Tum et 
leurs premières tentatives pour atteindre les hauts plateaux, afin d'échapper aux persécutions des années $1840^{6}$. En 1842, deux autres missionnaires français parviennent jusqu'aux plateaux par la route la plus accessible, en remontant le fleuve Ba qui traverse l'actuelle province de Phú Yên. Ils sont finalement capturés dans les zones montagnardes par des marchands Việt et livrés aux mandarins. D'autres tentatives dans les actuelles provinces de Quảng Ngãi et de Quảng Nam échouèrent de façon similaire, à cause toujours des relations étroites entre les marchands việt et les mandarins ${ }^{7}$.

En 1848, l'évêque français envoie un jeune catholique việt en mission au marché des sources d'An Sơn afin de trouver une route reliant les plateaux aux fleuves de la province de Bình Định. Dourisboure décrit An Sơn comme un "grand centre de commerce entre les Annamites et les sauvages " à partir duquel «les Annamites font [...] un commerce considérable chez les sauvages dont ils parcourent sans cesse les tribus, mais aucun d'eux ne peut songer à y fixer sa demeure. Les sauvages, de leur côté descendent pour leurs ventes ou achats jusqu'à An Son, sans jamais oser franchir cette limite ${ }^{8}$. " Il commente également brièvement l'importance politique passée de cet endroit: " C'est ce village qui a été, vers la fin du siècle dernier, le berceau de la révolte contre le roi Gia-Long. [...] Depuis cette mémorable époque, An-Son (jadis Tay-Son) inspire toujours des craintes à la politique soupçonneuse des rois d'Annam; aussi leurs lois défendent-elles, sous la menace des peines les plus sévères, aux Annamites de s'établir sur les terres des sauvages, et à ceux-ci de dépasser An-Son, pour entrer en Annam. "

Le programme du jeune catholique, dessiné par l'évêque, est le suivant: "Je me ferai marchand [...] et tout en faisant semblant de commercer, je m'avancerai dans l'intérieur jusque par-delà des limites que ne franchissent pas les autres marchands ${ }^{9}$. " Après avoir travaillé comme cuisinier pour un marchand việt, il devient marchand à son propre compte, apprenant la langue et la topographie des lieux, jusqu'à ce qu' « un jour, les sauvages résolurent de s'emparer de ses marchandises, qu'ils se figuraient devoir être très précieuses, et de le prendre, lui et ses gens, pour les vendre comme esclaves au Laos ${ }^{10}$ ». Ayant réussi à

\footnotetext{
6. Pierre Dourisboure, Les sauvages Ba-hnars (Cochinchine orientale) : souvenirs d'un missionnaire, Paris, de Soye, 1873 [449 p.].

7. Ces relations méritent à elles seule une étude, les marchands étant la plupart du temps des agents de commerce des autorités locales.

8. Ibid., p. 9

9. Ibid., p. 11.

10. Ibid., p. 14-15
} 
s'échapper, il informe l'évêque que la route des hautes-terres n'est pas fréquentée par les marchands vietnamiens.

Cette route est empruntée un an plus tard par deux missionnaires, Combes et Fontaine. À partir de Trạm Gò, sur la côte ouest de la plaine de Bình Định, ils voyagent jusqu’au village de Kon Phar: "Arrivés là, ils avaient dépassé la limite au-delà de laquelle ne s’avancent pas les marchands annamites, et ils pouvaient sans craindre prendre la direction du sud-ouest ${ }^{11}$ ". Avant leur départ, l'évêque les avait mis en garde contre le bourg d'An Sơn et leur avait bien recommandé d'éviter de croiser le chef d'un village bahnar nommé Kiêm: "Comme il parlait bien la langue d'Annam et faisait avec les marchands cochinchinois un grand commerce, ceux-ci le prenaient pour arbitre dans les différends qui s'élevaient entre eux et les sauvages. Sa supériorité était donc généralement reconnue, et les mandarins annamites, pour mieux le gagner et se servir de son ascendant, lui avaient procuré un diplôme. [...] Ils en avaient fait un agent dévoué du gouvernement annamite ${ }^{12}$ ".

Malheureusement, lors de leur arrivée à Kon Phar, « la première personne qu'ils y rencontrèrent fut le fameux Kiêm... "

Kiêm se révéla aussi puissant que les missionnaires l'avaient redouté, mais nettement plus favorable à leur entreprise que ce qu'ils avaient anticipé.

Quelques années plus tard, après l'établissement réussi de la mission de Kon Tum, un mandarin vietnamien du district de Tuy Viễn — district dans lequel se trouve An Sơn - devait rapporter à la cour quelques aspects de la vie sociale et économique de cette région. Le contenu du rapport est organisé par rubriques, sous divers intitulés administratifs: "Pratiques et coutumes ", "Production locale ", "Rivières et montagnes ", etc. Il contient des détails sur les montagnards au milieu desquels vivent les missionnaires et également des informations sur la villemarché d’An Sơn (nommée ici Phương Kiệu), que les pères catholiques traversent désormais sans craindre d’être capturés.

Pratiques et coutumes

Les montagnards des villages des hautes terres construisent des maisons sur pilotis et élèvent du bétail pour vivre (les habitants vivent sur les plates-formes, le bétail en-dessous). Ils attachent des ficelles pour établir des relations de confiance, se vêtent simplement et en fonction de leur activité, ne commettent pas de vol et aiment posséder des biens en

11. Ibid., p.27.

12. Ibid., p.29. 
bronze comme des plateaux, des marmites, des gongs et des récipients comme des jarres en terre cuite ${ }^{13}$.

Productions locales

Du bois de kiền-kiền, des casseroles, de la résine, des lampes à huile, du bois d'aigle, de l'ivoire, du miel d'abeille, de la cire d'abeille, de la teinture brune, des paons, des buffles et des chevaux sont issus des cantons de Phú Phong et de Nhơn Nghĩa, disponibles dans les marchés des sources et dans les hautes terres (en faible quantité) ${ }^{14}$.

Les rivières et les montagnes

Le marché des sources de Phương Kiệu: il fait partie du village de An Khê, situé sur la frontière ouest du canton de Phú Phong, dans le district du même nom. Ce territoire est empli de miasmes. Un fort y a été construit pour surveiller l'endroit. Auparavant, c'était un lieu d'échanges, comprenant une frontière et des officiers des douanes postés pour le superviser. An Khê était à l'origine la première ferme des [frères] Tây Sơn; le terrain est plat et étendu. Ce marché des sources se situe sur le fleuve Ba, qui est connecté avec la région des hautes terres à quelques lieux en amont du fleuve. Après quelques jours de voyage, on rencontre le fleuve Đôn. Les rivières ruissellent toutes vers le Sud et rejoignent le fleuve Đà Điễn, dans le comté de Phú Yên. Si l'on continue 12 ou 13 jours vers l'ouest, on atteint le fleuve Ba La, qui s'écoule vers l'ouest et rejoint le fleuve Khung Giang [Mékong]. Quelques fois, des chefs religieux provenant de l'Océan de l'Ouest [L'Europe] viennent ici et les chefs religieux de notre pays vivent ici pour enseigner leur religion aux montagnards ${ }^{15}$.

Une comparaison résumée de ces deux sources (le récit de Dourisboure et le rapport de mandarin du district de Tuy Viễn) nous donne les informations suivantes:

- Avant la fin du xviII ${ }^{\mathrm{e}}$ siècle, An Sơn est un lieu d'échanges important. Par la suite, sous la dynastie des Nguyễn, des restrictions au commerce,

13. Phong tục: Người Thượng ở các sách động làm nhà sàn để ở, làm nghề chăn nuôi súc vật (trên sàn người ở, dưới sàn súc vật ở'), kết giây làm tin, ăn mặc tiết kiệm, không ăn trộm của nhau, thích có các vật dụng bằng đồng như mâm, nồi, chiêng, công và đồ đựng như chỉnh sành.

14. Sản vật: Gỗ kiền kiền, soong, nhựa trám, dầu hoả, trầm hương, tốc hương, ngà voi, mật ong, sáp ong, củ nâu, chim công, trâu, ngựa sản ở 2 tổng Phú Phong, Nhơn Nghĩa, các nguồn và ở vùng người Thượng (đều không nhiều).

15. Sông núi : Nguồn Phương Kiệu : thuộc thôn An Khê ở địa giới phía tây tổng Phú Phong của huyện hạt. Đất quá nhiều lam chướng. Có dựng một đồn canh phòng. Trước đây là nơi buôn bán, đặt viên tấn thủ để trông coi. An Khê vốn là trại cũ của Tây Sơn, đất khá bằng phẳng rộng rãi. Nguồn này có sông $B a$, phía trên sông vài dặm tiếp liền với vùng người Thượng. Đi vài ngày đường lại có sông Đôn. Các sông đều chảy xuống phía nam, đổ vào sông Đà Diễn đạo Phú Yên. Lại đi về phía tây 12-13 ngày đến sông $B a$ La, chảy về phía tây rồi đổ vào Khung Giang. Thinh thoảng có đạo trưởng người Tây dướng đến, còn đạo trưởng người nước ta thì thường trú ở đây để dạy đạo cho người Man. 
portant autant sur l'importation que sur l'exportation, sont mises en place suite à la rébellion des Tây Sơn, dont An Sơn aura été le point d'origine. Au XIX siècle, les marchands Việt et montagnards continuent de se côtoyer, mais dans une moindre mesure qu'auparavant ;

- Les marchands việt entretiennent des relations personnelles avec les chefs montagnards afin de faciliter les relations commerciales ;

- Les produits forestiers circulent vers l'aval et les objets en bronze et en céramique circulent vers l'amont (il est à noter que le produit le plus prisé par les montagnards et provenant des plaines - le sel — est manquant dans la liste du mandarin) ;

- Il existe une limite territoriale que les marchands ne franchissent pas: elle se situe dans les montagnes pour les Việt et dans les plaines pour les montagnards. Cette pratique a été établie sous la dynastie des Nguyễn pour le marché d'An Sơn - politiquement sensible - et étendue aux pratiques commerciales des autres lieux d'échanges ;

- Le gouvernement vietnamien établit une présence militaire, par la construction d'un fort, l'instauration d'une frontière et la présence d'agents des douanes.

Ces documents nous donnent un aperçu de la situation du marché des sources dans la période suivant l'apogée de sa prospérité. Le déclin de ces marchés au XIX ${ }^{\mathrm{e}}$ siècle est bien documenté pour le cas d’An Sơn, particulièrement défavorisé pour des raisons politiques liées au fait que la dynastie des Nguyễn continue de craindre les Tây Sơn. Mais An Sơn n’est pas le seul marché à subir cette défaveur, partagée en effet par d'autres places commerciales et par le port de Hội An.

Comme l'indiquent ces documents, le marché des sources a plusieurs fonctions. Il est d'abord et avant tout une place d'échanges, connectée aux hautes terres et aux plaines par la rivière et les sentiers forestiers. C'est ensuite une zone frontière et un lieu de taxation des échanges transfrontaliers, muni du personnel ad hoc et d'un équipement militaire. Enfin, c'est une division administrative, l'équivalent d'un canton (tổng) dans les plaines: $\mathrm{au} \mathrm{XIX}^{\mathrm{e}}$ siècle, quatre-vingts villages des hauts plateaux (sách) sont énumérés comme étant sous la responsabilité du marché des sources (nguồn) de Phương Kiệu ${ }^{16}$. Les archives de la dynastie des Nguyễn listent sept marchés des sources dans la province de Bình Định, chacun comportant son poste

16. « Đồng Khánh Địa Dư Chí » (La géographie descriptive de l'empereur Đồng Khánh), section sur la province de Bình Định, district de Tuy Viễn. Manuscrit conservé à l'Institut des études Hán-Nôm, Hanoi. 
de contrôle frontalier et douanier (thủ sở), sa place d'échanges (trường giao dịch) et son fort (đồn bảo $)^{17}$. Leur fonction économique est primordiale: l'activité principale de ces postes est d'administrer la collecte des taxes sur le commerce tandis que le fort fournit un lieu sécurisé pour les marchandises et pour les revenus générés par le commerce.

Nous lisons, dans le premier chapitre de l'ouvrage Trên đất Nghĩa Bình, le récit d'une mission ethnographique conduite en 1977 où l'historien Nguyễn Quang Ngọc identifie les vestiges de ce marché des sources:

Le marché [trường] d'An Khê, dans l'actuel village d'An Luỹ, demeure encore, érigé il y a longtemps et reconstruit en 1968-1969 sur l'ancienne place du marché. La population locale explique que le mot trường signifie marché, place d'échange. Au sud du marché d'An Khê, il existe encore une zone de rizières de faible altitude nommée vulgairement le marché de Rộc, ainsi qu'un arbre ké nommé " l'arbre du marché ", qui s'élève à 30 mètres de haut pour une circonférence de 4,5 mètres. Il convient de signaler qu'à 300 mètres à l'ouest du marché d'An Khê, il subsiste encore aujourd'hui un monticule de terre, nommé « le tertre du marché », désigné par les locaux comme l'ancienne place du marché d’An Khê ${ }^{18}$.

Ces bourgs sont d'une indéniable importance économique au $\mathrm{xvIII}^{\mathrm{e}}$ siècle du fait des taxes que leurs marchés envoient à la cour de Phú Xuân, comme le démontrent les archives de Lê Quý Đôn. Ce dernier indique comme suit le revenu, collecté en 1774, de quatre marchés des sources de la province de Bình Định:

- Le marché des sources de Hà-nhiễu, dans la préfecture de Qui-nhân, doit payer une taxe annuelle s'élevant à 166 ligatures 300 sapèques $^{19}$, ainsi que la taxe thổ-nghi de 27 ligatures 300 sapèques; trois pots de miel, quatre rouleaux de nattes de rotin, 99 catties de coton, 3 sacs de ouates de coton et de soie d'un pesant chacun, 105 ligatures de sapèques, 25 pots de fruits de canarium, 60 plantes de rotin-dragon, 2 pots d'huile parfumée, 3325 grandes lampes, 60 petites lampes, 70 bols de cire d'abeille ;

17. Đại Nam nhất thống chí ("Géographie du Đại Nam unifié »), vol. 3, Huế, Nxb Thuận Hóa, 1997, p. 37-38.

18. Phan Huy Lê, Phan Đai Doãn, Vũ Minh Giang, Nguyễn Quang Ngọc, Trên đất Nghĩa Binh ( « Sur la terre de Nghĩa Bình »), Qui Nhơn, Sở văn hóa thông tin Nghĩa Bình, 1988, p. 16.

Thôn An Lũy hiện nay vẫn còn Anh Khê trường đước xây dựng từ lâu và được trùng tu lại vào năm 19681969 trên khu vực trường sở giao dịch ngày xưa. Nhân dân địa phương đều giải thích thống nhất là trường có nghĩa là trường sở buôn bán, trường giao dịch. Ở phia năm An Khê trường hiện nay còn khu ruộng trũng mang tên thô Rộc trường và cây ké Rộc trường cao 30 m chu vi 4,5 m. Đáng chú ýlà ở cách An Khê trường 300 m về phía tây, nay còn một gò đất cao mang tên gò chợ, theo nhân dân kểlại thì chợ An Khê trước đấy.

19. Monnaie vietnamienne: 1 ligature (quan) $=10$ tiền; 1 tiền $=60$ sapèques (ici - chũ). Donc 1 ligature $=600$ sapèques 
- Le marché des sources de Trà-đinh et le marché des sources de Trà-vân doivent payer une taxe annuelle de 2550 ligatures de sapèques. Auparavant, la taxe des marchés des sources de Trà-đinh et de Trà-vân servait à payer le salaire du mandarin de droite intérieur nommé Khoan, et le marché des sources de Cầu-bồng devait payer 11 thoi 2 lượng ${ }^{20}$ d'argent de la meilleure qualité ;

- Les autres marchés des sources de la province sont ceux de Đá Bàn, qui paie une taxe de 1000 ligatures de sapèques et de Ô Liêm, qui paie une taxe de 749 ligatures 300 sapèques. Plus au nord, les revenus sont tout aussi impressionnants: dans la province de Quảng Ngãi, ils s'échelonnent de 610 ligatures de sapèques au marché des sources de Bà Địa à 3000 ligatures pour celui de Cù Ba Cây Mít, et dans la province de Quảng Nam, de 198 ligatures à Đông Lỗ à 2060 ligatures à Chiên Đàn. Il faut également noter que le montant collecté dans les régions ayant pour principale ressource le bois d'aigle, comme au marché des sources de Nha Trang, est multiplié par trois, à savoir 10000 ligatures.

Un rapide et simple calcul, basé sur les chiffres de Lê Quý Đôn pour l'année 1774, indique que les recettes fiscales monétaires des marchés des sources de la région entière (34716 ligatures) équivalent à presque la moitié des revenus en monnaie provenant de tous les étangs, les montagnes et les marchés (76467 ligatures). Cela représente une proportion considérable de l'économie commerciale du pays ${ }^{21}$. Cette somme peut également être comparée au montant des taxes collectées sur l'exportation (souvent des mêmes marchandises) au port de Hội An, qui atteint 30800 ligatures. Une comptabilité complète requiert des calculs bien plus complexes: en effet, la majorité des taxes de ces marchés sont payées en

20. Lê Quý Đôn, Phủ biên tạp lục ("Écrits divers sur les districts frontaliers »), Saigon, Phủ quốc vụ khanh đăc trách văn hoá, 1972, vol. 2, p. 30.

- Nguyên Hà-nhiễu thuộc phủ Qui-nhân hàng năm phải nạp số tiền thuế là 766 quan 5 tiền, cùng thuế thố-nghi 27 quan 5 tiền, mật-ong 3 tĩn, chiếu mây hạng nhỏ 4 cuốn, bông gòn 99 cân, kỳ-hoa miên-hoa 3 bao cân nặng 105 quan tiền, cảm-lãm 25 lâu (sọt), long-đằng 60 cây, dầu thơm 2 tĩn, đèn lớn 3325 chiếc, đèn nhỏ 60 chiếc, sáp-ong 70 bát.

- Nguyên Trà-đinh và nguyên Trà-vân hàng năm phải nạp số thuế là 2250 quan tiền. Trước đây, tiền thuế nguyên Trà-đinh và nguyên Trà-vân được dành để cấp ngụ-lộc cho quan Ngoại-hữu Trươngphúc-Loan, nên hai nguyên nầy phải nạp 5 thoi giáp-ngân.

- Nguyên Trà-vân còn phải nạp riêng 590 cây đèn dầu cảm-lãm để cất chứa vào kho dầu.

- Nguyên Cầu-bông [=An Sơn] hàng năm phải nạp số thuếlà 750 [0] quan. Trước đây, số thuếnguyên Cầu-bông đượ dành để cấp lương cho quan Nội-hữu-chưởng tên là Khoan, nên nguyên Cầu-bông nầy phải nạp 17 thoi 2 lượng giáp-nhân.

21. En 1774, les recettes collectées à partir des marchés des sources de la province de Bình Định (6,255 quan tiền) représentent à eux seuls $8 \%$ du revenu collecté à travers tout le territoire des Nguyễn à partir des montagnes, des étangs, des passages et des marchés $(76,467$ quan 2 tiền 54 chũ). Ibid., p. 9. 
biens et non en espèces monétaires. Ces biens ont une grande valeur mais leur conversion en équivalent monétaire est un exercice bien incertain. À cela s'ajoute le fait que l'année 1774 est une année exceptionnelle car elle est une des dernières années de la fin de la crise du régime des seigneurs Nguyễn, durant laquelle ces marchés sont considérablement surtaxés.

Cependant, cette lecture superficielle des chiffres de la compilation de Lê Quý Đôn indique que, même si nous prenons en compte les troubles économiques et les taux anormaux d'imposition de cette période, la taxation des échanges entre les hautes terres et les basses terres dans les marchés des sources représente une proportion très importante des ressources du régime des Nguyễn.

\section{Les relais}

Ce type de marché, comme nous l'avons vu, est un élément central dans le modèle commercial amont-aval. Sa centralité est d'ailleurs renforcée à travers ses fonctions de principal lieu de contact entre les différents groupes ethniques: il s'agit du principal point de passage de témoin dans le système de relais qui anime le commerce est-ouest dans le modèle économique post-Champa. Même si certains montagnards prennent part aux marchés côtiers, ce sont ces centres de relais qui sont primordiaux pour leurs relations commerciales avec les Việt, les Chinois et les Cham.

Nous l'avons vu, un récit détaillé sur le marché des sources d'An Sơn est livré par le premier chapitre de Trên đất Nghĩa Bình, qui contient de riches données ethnographiques et d'autres détails intéressants. Un article de 1906 écrit par un officier militaire colonial posté à An Sơn, Charles-Marie Trinquet, contient également une description de cette zone frontalière ${ }^{22}$. Dans ces deux sources datant du xx $x^{e}$ siècle, le plateau de An Khê est habité par des populations bahnar et việt, qui entretiennent des relations commerciales: les marchandises circulent entre les populations việt des plaines, et les Bahnar et d'autres groupes ethniques de la région montagneuse.

Pour comprendre un environnement commercial plus complexe, il convient de s'intéresser à une place d'échanges située plus au Nord, au marché des sources de Trà My dans l'actuelle province de Quảng Nam, autrefois centre majeur de collecte de cannelle ${ }^{23}$. Au début du xx siècle,

22. Charles-Marie Trinquet, "Le plateau d'An-Khé», Revue Indochinoise, 1906, p. 1061-1070, 1152-1165.

23. Ce lieu était connu à l'époque de Lê Quý Đôn comme Chiên Đàn. Lê Quý Đôn, Phủ biên tạp luc, vol. 2, p. 29. 
un auteur français fait les observations suivantes, après une présentation des résultats de son étude détaillée de la collecte et de la préparation des produits forestiers de haute valeur marchande:

Des manipulations aussi compliquées nécessitent un personnel considérable et, de fait, toute la population habitant les marchés de cannelle est, aux saisons de la vente, tout entière occupée à les exécuter. En dehors de ces époques, où une réelle activité se manifeste dans les centres d'échanges, la population de ces villages retourne à ses rizières et à ses jardins. Le plus important de ces centres est le marché de Tra-my. Rien de plus coquet que ce village blotti dans le fond de la vallée du Sông-Tan [Sông Tranh], à l'abri de superbes jardins tout plantés d'aréquiers, de poivriers, de théiers, etc. Tout autour se développe un énorme cirque de montagnes boisées. Malheureusement, si le pays est riche, il est aussi malsain. Quand on visite Tra-my, on est frappé de l'air misérable des habitants, malgré l'admirable fertilité du terrain qu'ils cultivent. La fièvre mine sourdement cette population, adonnée presque toute entièrement à l'opium, persuadée que l'usage de cette drogue la préserve, dans une certaine mesure, de l'influence délétère de ce climat de montagne. Les habitations sont pourvues d'un double toit, le premier en paillote, le second en terre et en chaux destiné à protéger l'intérieur des incendies allumés par les Moïs. De temps à autre, une incursion de ces derniers se produit; quelques habitants sont emmenés en esclavage; néanmoins la population est en voie d'augmentation lente, les habitants de la plaine étant peu à peu attirés par l'appât des bénéfices réalisés par la cannelle ${ }^{24}$.

Une mission de terrain menée dans cette même zone, en 2007-2008, a livré plusieurs informations sur la route que suivait la cannelle, à partir des plantations dans les montagnes, situées à la portée de ce bourgmarché et cultivées majoritairement par les populations de l'ethnie Co, jusqu'au port de Hội An. Les planteurs de l'ethnie Co amènent la production au marché périodique de Trà My ou la vendent aux marchands việt qui travaillent pour des collecteurs chinois et viennent dans les villages Co. À partir du centre de récolte de Trà My, la marchandise est transportée jusqu'aux rues chinoises ( $p h o ̂ ́)$ de Tam Kỳ — en passant par des chemins forestiers - d'où elle est alors chargée sur des jonques et transportée sur la rivière jusqu’à Hội $\mathrm{An}^{25}$.

24. Brière, "Culture et commerce de la cannelle en Annam », Bulletin économique de l'Indochine, $n^{\circ} 33$, septembre 1904, p. 945-946 [935-950].

25. Recherches de terrain menées dans le district de Trà My, province de Quảng Nam, juin 2007, et dans le district de Nam Trà My, province de Quảng Nam, mars 2008. 
Comme le remarque Lê Quý Đôn, Hội An est le principal port de la région qui exporte non seulement des produits provenant des hautes terres de la province de Quảng Nam, mais également des produits provenant de plusieurs marchés des sources de la région:

Concernant la majorité des biens produits dans les préfectures de Thănghoa, Điện-bàn, Qui-nhân, Quảng-nghĩa et Bình-khang et dans le territoire de Nha-trang, il y a des endroits où les populations transportent des biens le long des routes fluviales, ou à cheval par voies terrestres, et tous les biens arrivent et se concentrent dans une rue de Hội- $\mathrm{An}^{26}$.

Les résultats de l'enquête de terrain menée plus au sud, dans la province de Quảng Ngãi, confirment cette information ${ }^{27}$. Jusqu'au xx ${ }^{\mathrm{e}}$ siècle, le port de Thu Sà, près de l'estuaire des fleuves Trà Khúc et Vệ, est habité principalement par des marchands chinois qui expédient des marchandises de l'intérieur des terres vers la côte, puis de ce port jusqu'à Hội An, vers le Nord du Vietnam ou jusqu'à Hong Kong. En amont — au marché des sources de chaque fleuve - jusqu'à l'extrémité ouest de la plaine côtière, les représentants chinois des maisons de commerces de Thu Sà et les agents des douanes việt emploient des agents commerciaux việt pour collecter les marchandises des Hrê dans les montagnes; la transaction s'effectue dans les environs de la muraille et, lorsque la sécurité le permet, au marché des sources ou dans les villages des Hrê. Plus à l'Ouest, le commerce est dirigé par les membres des ethnies Ca Dong et Sédang. Ce dernier groupe descend régulièrement des hauts plateaux pour vendre des gongs et d'autres marchandises provenant du Laos. L'ensemble des intermédiaires consiste, d'est en ouest, en Chinois, Việt, Hrê, Ca Dong, Sédang et Lao, chacun gérant une portion spécifique de la route commerciale.

$$
* * *
$$

Comme cela a été noté, au Champa comme en d'autres lieux d'Asie du Sud-Est, les populations montagnardes travaillent pour les royaumes des plaines en tant que relais politiquement autonomes, approvisionnant la

26. Lê Quý Đôn, Phủ biên tạp lục, vol. 2. p. 72. Đại phàm những hóa-vật được sản xuất từ các phủ Thăng-hoa, Điện-bàn, Qui-nhân, Quảng-nghĩa, Bình-khang cùng dinh-sở Nha-trang, chỗ thì người ta chuyên-chở hàng-hoa bằng thuyền theo đường thủy, hay chở bằng ngựa theo đường bộ, đều đổ sô đồn về phố Hội an cả.

27. Recherches de terrain menées dans le district de Sơn Hà, province de Quảng Ngãi, 20092012. 
côte en produits forestiers, d'où ils étaient alors revendus comme biens de luxe dans les réseaux commerciaux internationaux d'Asie de l'Est. Après le déclin du Champa, un nouveau modèle d'organisation sociale émerge dans les plaines du centre, avec l'établissement de migrants vietnamiens côtoyant des populations indigènes déjà présentes. Un nouveau système économique basé sur la riziculture s'installe, modèle qui se rapproche davantage du système vietnamien du Nord. Mais durant cette période de transition - du xvi ${ }^{\mathrm{e}}$ au xviII ${ }^{\mathrm{e}}$ siècles - les deux modèles coexistent.

Ce système économique hybride est un facteur primordial du succès et de la longévité du régime des seigneurs Nguyễn. La richesse engendrée par l'exportation de produits forestiers a permis à cette dynastie de mener la lutte avec ses rivaux du Nord, les Trịnh, et de financer l'expansion du royaume vers le Sud. Ensuite, au cours du xix $x^{e}$ siècle, le modèle vietnamien prend son essor; le modèle post-Champa survit, désormais affaibli, à travers un système d'échanges amont-aval d'échelle uniquement locale. C'est durant cette période que la région côtière du Vietnam s'est dotée de caractéristiques économiques qui subsistent encore aujourd'hui, avec notamment son relatif désavantage économique par rapport aux deltas du Nord et du Sud.

L'importance de ce système des marchés des sources, pour les seigneurs Nguyễn, est soulignée par les circonstances de la disparition de leur régime. Au moment de la rébellion des Tây Sơn, le régime connaît en effet un état de crise politique croissant depuis plusieurs décennies. C'est une erreur, selon nous, de considérer cette rébellion comme une cause fondamentale d'effondrement du régime. Le soulèvement des frères Tây Sơn doit plutôt être considéré comme la touche finale de l'effondrement d'un édifice depuis longtemps vermoulu ${ }^{28}$.

L'aîné des frères Tây Sơn, Nguyẽn Nhạc, exerce, au milieu du XVIII ${ }^{\mathrm{e}}$ siècle, deux professions au marché des sources dénommé (selon les textes) Tây Sơn/An Sơn/An Khê/Phương Kiệu/Cầu Bông. Il pratique le commerce avec les montagnards et prélève les taxes sur le commerce pour les autorités du marché. La surtaxation du commerce amont-aval et les autres effets du dysfonctionnement administratif de l'époque ont un impact certain sur la prospérité du commerce; le système économique post-Champa subit alors des pressions considérables. La rébellion de Nguyẽn Nhạc est donc un symptôme économique de cette pression politique. Si le fait que le soulèvement des Tây Sơn débute dans un des

28. Le mot «vermoulu » est de Léopold Cadière. Voir son «Quelques figures de la cour de Vo-Vuong », Bulletin des amis du vieux Hué, 4, 1918, p. 271 [253-306]. 
Le Vietnam - Une histoire de transferts culturels

principaux marchés des sources de la région n’est pas une coïncidence, c'est, pensons-nous, parce que les marchés des sources jouaient un rôle considérable dans l'économie hybride qui a émergé de la rencontre entre les civilisations cham et vietnamienne, et a ainsi permis de soutenir le régime des seigneurs Nguyễn pendant deux siècles. 


\section{7}

\section{Des orientalistes en Orient}

\section{L'École française d'Extrême-Orient au Vietnam (1902-1957) \\ Pierre-Yves Manguin}

Fondée entre 1898 et 1901, à Saigon puis à Hanoi, l'École française d'Extrême-Orient (EFEO), institution académique, s'est trouvée d'emblée placée dans une position cruciale de passeur culturel. Elle a eu pour mission tout à la fois d'étudier sur place, sur un mode universitaire, les cultures de l'Asie orientale et de mettre en valeur un espace culturel plus spécifiquement Sud-Est asiatique, celui de l'Indochine française ${ }^{1}$. Le désir de créer une société savante en Indochine a pris naissance chez un groupe pionnier d'administrateurs coloniaux, de militaires et de missionnaires, passionnés d'arts et de traditions indigènes, de la conservation de monuments anciens et de l'étude des langues vivantes, tous domaines jusque-là négligés en Indochine française. Leurs démarches auraient pu aboutir à la création d'une association modelée sur la prestigieuse Asiatic Society des Indes britanniques, ou sur la déjà ancienne Société batavienne des arts et des lettres des Indes néerlandaises; l’EFEO aurait dès lors fonctionné dans un cadre résolument colonial. Présentée pour la première fois au congrès des orientalistes de 1897, cette initiative a été dès lors, en quelque sorte, confisquée par des savants universitaires, pour la plupart membres de l'Académie des inscriptions et belles-lettres. Les indianistes Auguste Barth et Abel Bergaigne, qui venaient de publier le premier recueil d'inscriptions sanskrites du Cambodge et du Vietnam, parvinrent à convaincre le ministre de l’Éducation de la nécessité de créer une nouvelle institution;

1. Pour les études utilisées pour écrire cet article, on se reportera à la liste donnée en annexe. 
Sylvain Lévi, autre indianiste éminent, en mission en Asie, réussit pour sa part à convaincre l'administration coloniale du bien-fondé de cette proposition. Le nouveau gouverneur général Paul Doumer adopta le projet: esprit éclairé convaincu de l'effet salutaire de l'éducation et bien disposé envers le monde savant, il comprit l'intérêt que l'on pouvait trouver à étudier les civilisations orientales sur leur propre sol. Il créa d'abord une mission archéologique permanente à Saigon en 1898, chargée de la gestion du patrimoine de l'Indochine, mais qui prit le nom d'École française d'Extrême-Orient l'année suivante, en se voyant dès lors attribuer un programme bien plus ambitieux, puisqu'il s'agissait d'étudier l'ensemble des civilisations de l'Asie orientale, et plus seulement celles de l'Indochine française. C'est ainsi que l'EFEO fut fondée: loin du monde académique métropolitain, institution indochinoise de recherche et de gestion du patrimoine, au sein de laquelle l'expérience du terrain et l'érudition pouvaient être combinées sur une base durable.

\section{Une mission ambiguë: une institution académique dans un contexte colonial}

La fondation de l'EFEO est éloignée d'une décennie à peine de la pacification de l'Indochine française. Dès le départ, l'histoire de l'école s'était ainsi trouvée intimement mêlée à celle, tourmentée, de cette région. La nouvelle institution ne pouvait que devenir indochinoise, et elle le restera de fait jusqu'à ce que, après les accords de Genève, dans un processus qui dura de 1955 à 1958, son administration soit transférée dans le système universitaire métropolitain, pour devenir l'une des cinq écoles françaises à l'étranger du ministère de l'Éducation nationale, placées sous le contrôle de l'Académie des inscriptions et belles-lettres.

Du fait de ces origines, l'EFEO bénéficiait par principe de l'autonomie académique attachée à l'enseignement supérieur. Cette liberté de recherche, dans ses domaines spécialisés, ne lui fut jamais contestée, et ce d'autant plus que son programme scientifique lui enjoignait de consacrer aussi ses recherches à des régions de l'Asie qui n’étaient pas sous la domination coloniale française. Cependant, sa création en Indochine signifiait que son budget lui était attribué par le gouvernement général de cette colonie et que ses membres devenaient de facto des fonctionnaires coloniaux, perdant au passage une part de la liberté attachée en métropole à l'enseignement supérieur (l'EFEO prendra un statut strictement académique à son implantation en France dans les années 1950). Son directeur était donc nommé par le gouverneur général, mais sur la seule 
recommandation de l'Académie des inscriptions et belles-lettres. La personnalité morale et l'autonomie financière qui lui furent accordées en 1920 par le gouverneur général renforcèrent encore son indépendance et celle-ci fut consacrée par Paul Doumer, devenu entre-temps président de la République ${ }^{2}$.

L'ambiguïté de son statut n'en est pas moins patente ${ }^{3}$. Les attributions de l'école ne sont pas exclusivement scientifiques, puisqu'elles englobent aussi des responsabilités techniques et administratives qui l'amènent à remplir les fonctions d'un service fédéral indochinois: chargée d'assurer l'inventaire, l'entretien et la restauration des monuments historiques, il lui revient à ce titre de veiller à l'exécution des arrêtés de l'administration coloniale qui assurent la sauvegarde de ces derniers ou encore d'organiser et diriger les musées indochinois.

Les hommes politiques fondateurs de l'école, tels Paul Doumer et ses administrateurs, attendaient bien sûr de son corps de scientifiques qu'il produise des connaissances qui soient dans l'intérêt du gouvernement colonial. Louis Salaün, proche collaborateur de Paul Doumer, a ainsi pu écrire que l'EFEO doit être l'un de ses guides:

Le travail de l'école intéresse directement notre politique indochinoise [...]. L'exercice du pouvoir protecteur en Indo-Chine [...] risquerait de tomber dans les fautes ordinaires aux pouvoirs absolus s'il n'était continuellement et prudemment guidé par le seul guide qui soit sûr, la connaissance scientifique du pays et des hommes dont il a la charge. L'école est donc, par la force même des choses, un collaborateur immédiat du gouvernement ${ }^{4}$.

Mais l'administration coloniale n'a pas toujours eu en Indochine des positions éclairées et elle a déploré la porosité de la frontière qui séparait les intérêts strictement scientifiques des membres de l'école et leurs réactions de citoyens à l'actualité immédiate; surtout quand elles lui étaient peu favorables et se trouvaient imprimées noir sur blanc dans la Chronique du Bulletin de l'école. Elle ne goûtait guère le libéralisme de certains membres de l'école à l'égard des indigènes. On connaît bien l'épisode de la censure qu'a tenté d'imposer en 1908 à Claude-Eugène

2. Le contexte administratif de la création de l'EFEO est décrit en détail dans Clémentin-Ojha et Manguin, op. cit., p. 33-38; Singaravélou, op. cit. p. 66 sq., et Lorin, Paul Doumer..., p. 93 sq.

3. J'emprunte bien sûr cette notion d'ambiguité de la situation coloniale indochinoise au livre de Pierre Brocheux et Daniel Hémery, Indochine, la colonisation ambiguë: 1858-1954. Paris, La Découverte, Syros, 2001.

4. Louis Salaün, L'Indochine. Paris, Imprimerie nationale, 1903, p. 354-355 (cité dans Lorin, op. cit., p. 95). 
Maitre, directeur de l'école, le gouverneur général qui reprochait à cette Chronique sa trop grande attention aux revendications politiques qui travaillaient l'Inde, la Chine et le Japon, et surtout l'Indochine ${ }^{5}$. L'épisode se termina à l'avantage de l'EFEO, avec les excuses d'un nouveau gouverneur général, mais il dénote le peu d'unanimité de la classe politique visà-vis du rôle à jouer par l'école. Il n'en reste pas moins que la Chronique perdit dès lors presque tout caractère politique, l'autocensure prenant le pas sur le contrôle politique de l'administration.

Deux citations, toutes simplificatrices et pompeuses qu'elles soient, illustrent mieux qu'un long exposé les positions distinctes des politiques et des scientifiques; elles sont tirées des deux discours prononcés lors des célébrations à Hanoi en 1951 du cinquantenaire de l'EFEO, à une époque où s'annonce la fin de la colonisation de l'Indochine et donc aussi la fin de la phase indochinoise de l'histoire de l'EFEO 6 . Il revient bien sûr au hautcommissaire de France et commandant en chef des armées, le maréchal de Lattre de Tassigny, d'y vanter la mission civilisatrice de la France et d'y associer l'EFEO:

Cinquante ans d'école française, cinquante d'efforts au service des pays d'Indochine pour les aider à être connus du monde, à se connaître euxmêmes. La science de l'école a magnifié comme un poème les gloires de ce pays. Si — pour ne reprendre qu'un exemple — le nom du Cambodge est désormais pour l'univers associé à la grandeur d'Angkor, c'est bien l'école française qui fut le trait d'union.

Les chercheurs de l'EFEO étaient-ils prêts, individuellement, à se ranger sous cette bannière en admettant avoir joué un tel rôle, décrit en termes aussi directs que subjectifs? Rien n'est moins sûr. Louis Malleret, qui était alors directeur de l'école, dans son propre discours lors de cette même cérémonie, ne pouvait bien évidemment pas faire part brutalement à cette occasion de sa perception divergente du travail effectué par l'EFEO. Il s'abstint donc de se lancer dans des considérations politiques, mais parvint plutôt par des voies obliques à évoquer "l'étendue du labeur accompli par l'école en un demi-siècle d'action désintéressée ", une mission d'universitaires sans motivations extra-académiques:

[l'EFEO] n'a eu d'autre fin que de porter à la connaissance du monde l'originalité des cultures qu'elle s'attachait à promouvoir sur un plan universel.

5. Clémentin-Ojha \& Manguin, op. cit., p. 35.

6. Louis Malleret, Le cinquantenaire de l'École française d'Extrême-Orient. Compte-rendu des fêtes et des cérémonies. Paris, Hanoi, E. de Boccard, EFEO, 1953, p. 20, 44. 
[...] une œuvre qui n’a connu ni artifices, ni voies obliques, ni réticences, ni calculs, ni aversions, ni mépris, mais qui, semblable au fleuve sollicité par l'appel de la mer, est allée droit à l'unité de la nature humaine.

À ces ambiguïtés politiques qui entourent la mission de l'école, on doit ajouter une autre situation paradoxale, qui est celle de la création même de l'EFEO en Indochine alors que la puissance coloniale vient de rayer de la carte plusieurs souverainetés nationales. La contradiction n’échappe pas aux membres de l'EFEO, qui l'expriment avec lucidité dans les pages mêmes du BEFEO de 1921, à l'occasion d'un survol de l'histoire de l'institution jusqu'en 1920 (p. 43):

Le seul fait de la présence en Extrême-Orient des Européens qui rend ces études possibles, est un risque grave de disparition pour leur objet: nos civilisations d'Occident semblent exercer une action dissolvante sur les civilisations propres à l'Orient, et nombre de formes présentes, nécessaires à l'intelligence des formes anciennes, seront, dans un avenir prochain, un passé mort.

Si l'on considère la formation orientaliste des membres de l'EFEO, on ne peut enfin pas douter qu'ils aient reconnu l'irréductible originalité des civilisations de l'Asie, à l'instar de maints politiques plus clairvoyants, tel le gouverneur général Pierre Pasquier, fin connaisseur du Vietnam, qui, en 1930, s'interrogeait sur les liens qu'il était possible de tisser entre les descendants de barbares gaulois civilisés par Rome, non sans résistances d'ailleurs, et les populations de l'Asie, dont les âmes et les esprits ont été moulés par «la plus ancienne civilisation du monde » ${ }^{7}$.

Il est ainsi difficile, sans avoir mené une étude approfondie - probablement irréalisable - de la correspondance privée des membres de l'EFEO, de savoir quelle était la position personnelle de chacun d'entre eux. Même si, comme a tenté de le démontrer Singaravélou, ils étaient dans la majorité issus de milieux privilégiés (en cela de purs produits de l’Université française...), ils n'en avaient pas moins des origines et des parcours éducatifs divers, plus que leurs confrères des écoles de Rome ou d'Athènes, comme on peut en juger par les quelques témoignages et les bribes de correspondance que nous avons pu consulter, par leurs biographies, sans parler des conflits qui les opposèrent parfois entre eux (telle la vive polémique lancée dans les années 1930 par Émile

7. Sur ces administrateurs éclairés, proches de l'EFEO pour certains, voir Patrice Morlat, «Projets coloniaux et mise en pratique : la politique des « fils » de Sarraut en Indochine dans les années vingt », Cahiers d'histoire, Revue d'histoire critique 85, 2001, p. 13-28. La citation complète de Pierre Pasquier est tirée de Brocheux et Hémery, op. cit., p. 218. 
Gaspardone) $)^{8}$. L'EFEO n'a jamais constitué un bloc monolithique prompt à défendre une quelconque idéologie. En Indochine, l'école a fonctionné dans un contexte historique qui a lui-même rapidement évolué, des premières années de la pacification à Dien Bien Phu et aux accords de Genève. Son personnel scientifique et ceux qui l'ont accompagné, des hommes pour la plupart, ont vécu deux guerres mondiales, la montée des nationalismes chez les peuples colonisés d'Indochine, la mise en place du régime de Vichy en Indochine, une relation malaisée avec un allié japonais devenu occupant après le coup de force de mars 1945, et pour finir une guerre coloniale au Vietnam. Des savants sont morts dans la résistance à l'ennemi allemand (Henri Maspero), ou des mains de l'occupant japonais (George Groslier), d'autres ont collaboré avec le Japon (Victor Goloubew). Sous Vichy, certains ont été exclus ou empêchés de rejoindre l'école en raison de leur origine juive (Suzanne Karpelès, Rolf Stein). D’autres avaient des sympathies communistes affichées (Paul Lévy, directeur de l'EFEO de 1947 à 1949), ou ont joué un rôle important pour tenter d'éviter la guerre coloniale (Paul Mus, qui sera plus tard un opposant notable à la guerre américaine au Vietnam, comme le sera aussi Georges Condominas). Dans des conjonctures historiques aussi variées que violentes, avec des marges de manœuvre fluctuantes, les convictions politiques, les comportements adoptés à l'égard du processus colonial et enfin les pratiques professionnelles ne peuvent qu'avoir été multiformes, selon l'époque ou l'individu. Ils ont certainement évolué au fil des décennies, comme le reste de la nation française à laquelle appartenait ce personnel scientifique, avec le développement inexorable, au cours du $\mathrm{xx}^{\mathrm{e}}$ siècle, d'une opinion explicitement anticolonialiste.

Ces différences ne peuvent qu'avoir marqué les attitudes individuelles et celle de l'institution vis-à-vis du peuple vietnamien et la façon dont les savoirs ont circulé, dans un sens comme dans l'autre. Dans le cadre très limité de cet article, on doit donc se tourner vers une analyse sommaire des pratiques de l'institution indochinoise dans sa globalité et, plus spécifiquement, de ce qu’elle a légué au Vietnam. Cet héritage est bien connu. Il est, comme toute œuvre scientifique, un produit de son temps. L'ambiguïté du statut de l'EFEO dans ce contexte colonial commande que ce grand œuvre orientaliste fasse l'objet, à tout le moins, de relectures

8. Cette polémique et les désaccords d'un Paul Mus, plus sociologue qu'orientaliste, sont évoqués dans Clémentin-Ojha et Manguin, Un siècle..., p. 115, et dans P.-Y. Manguin, « Un "sociologue" parmi les orientalistes: Paul Mus à l'École française d'Extrême-Orient (1927-1937) », in L'espace d'un regard: Paul Mus et l'Asie (1902-1969), Christopher Goscha and David Chandler (éd.), Lyon, Paris, Institut d'Asie Orientale, Les Indes savantes, 2006, p. 109-116. 
attentives aux circonstances de son écriture. Le versant patrimonial des activités de l'école - restauration et conservation des monuments, création de musées - a évolué lui aussi au cours de la première moitié du $\mathrm{xx}^{\mathrm{e}}$ siècle et, comme sa production scientifique, reflète un discours bien de son temps sur les civilisations qu'il met en exergue.

\section{Le legs de l'EFEO au Vietnam}

L'enseignement au niveau universitaire du sanskrit, du pâli et de l'archéologie était inscrit par l'Académie parmi les tâches dévolues à l'EFEO en Indochine, de telle sorte « qu'entre savants d'Europe et lettrés d'Asie un rapprochement se fera, qui contribuera au progrès matériel et moral de la colonie ». Mais cet enseignement formel fut vite rejeté par l'EFEO. En réponse à ces suggestions, son directeur Louis Finot, fut prompt à répondre, lors d'une séance de l'Académie, en mai 1901:

Notre intérêt est de nous tenir fermement à l'objet principal de notre fondation: cet objet n'est pas de répandre les vérités connues, mais de découvrir des vérités nouvelles; ce n’est pas l'enseignement, c’est la recherche ${ }^{9}$.

En Indochine, néanmoins, un enseignement de langues a parfois été assuré par l'école. En 1907, par exemple, on peut y étudier la langue chinoise parlée et écrite, le sanskrit, le tibétain et le japonais. À l'époque, l'institution se félicite de ne favoriser que les études désintéressées, qui ne préparent à aucun de ces brevets avec prime conférés en Indochine aux administrateurs, prenant là aussi ses distances par rapport au pouvoir colonial. Elle se propose aussi de prêter son concours actif à la réforme de l'enseignement indigène, en encourageant ses membres à participer à la vie de l'Université indochinoise, créée en 1918, et contribuer ainsi à l'émergence d'une élite locale. Mais ce programme idéal n'est pas suivi d'effets concrets. Dans les années 1940, cependant, le vent tourne et l'EFEO reprend ses cours, sous l'impulsion en particulier d'un directeur, Paul Lévy, qui insiste pour associer les Vietnamiens aux travaux de l'école. Dès lors, la palette des matières enseignées par ses membres s'enrichit: elle reflète désormais aussi leurs recherches dans les domaines

9. Sur la mission éducative de l'EFEO et le peu d'enthousiasme mis par ses membres pour la mettre en pratique, on lira la lettre de Michel Bréal, de l'Académie des inscriptions et belles lettres, publiée dans le Bulletin de l'EFEO de 1901 (p. 7-8: "Mes souhaits pour l'École française d'Extrême-Orient ») et la réponse de Louis Finot, publiée au même BEFEO de 1901 (p. 385). Singaravélou a noté ces désaccords (op. cit., p. 71-75). 
de l'histoire et de l'anthropologie de l'Indochine. Les membres de l'EFEO, pour diffuser leur savoir, prononceront certes aussi en Indochine des conférences auprès d'un public éduqué, dont on ne connaît pas bien la composition, mais dont on peut imaginer qu'il était surtout fait de l'élite de la colonie, d'origine française pour l'essentiel (les Cahiers de l'EFEO, publiés dans les années 1930 et 1940, donnent des résumés de ces conférences).

Des trois fonctions attribuées à l'école, l'enseignement en Indochine est donc bien celle qui suscite en son sein le plus de réticences. Les membres de l'EFEO ont été nombreux à assurer un véritable enseignement universitaire, mais ils l'ont fait en France, en quittant l'Indochine pour intégrer le personnel scientifique de l'école nationale des langues orientales, et surtout de l'École pratique des hautes études et du Collège de France. Il résulte de ces réticences qu'après quelque cinquante années de présence en Indochine l'héritage majeur laissé par l'EFEO est bien constitué de la production scientifique de ses membres, plutôt que d'une impérieuse mission civilisatrice.

Cette première période indochinoise de la vie de l'École française d'Extrême-Orient se clôt, symboliquement, par l'élaboration par l'épigraphiste George Cœdès de la première grande synthèse sur l'histoire de l'Asie du Sud-Est indianisée, intitulée Les États hindouisés d'Indochine et d'Indonésie (seules les cultures non vietnamiennes présentes sur le territoire vietnamien y sont donc abordées). Son titre et ses commentaires interprétatifs, à quelques mises à jour près, sont arrêtés dans sa deuxième édition de 1948. L'édition révisée de 1964 fera l'objet d'une traduction anglaise (revue par l'auteur) en 1968, largement diffusée et citée depuis. Fondé pour l'essentiel sur l'immense œuvre accomplie par les épigraphistes et les archéologues français et néerlandais, cet ouvrage fait indéniablement date et sert aujourd'hui encore, à bien des égards, de manuel de référence. Grand œuvre incontesté de l'orientalisme pour sa partie factuelle, il faudra attendre les années 1990 pour que sa vision de l'apport civilisateur de l'Inde - perçu à bien des égards comme une première mission civilisatrice — soit remise en cause par de nouveaux paradigmes, soutenus tout à la fois par les découvertes nombreuses d'une archéologie plus rigoureuse et par une vision renouvelée, postcoloniale, de l'histoire de la région ${ }^{10}$.

10. Sur les perceptions des rapports à la Chine ou à l'Inde de l'école orientaliste, voir entre autres Bernard Formoso (" L'Indochine vue de l'Ouest», Gradhiva, 4, 2006, p. 35-51) ; et l'introduction de l'auteur du présent article à l'ouvrage collectif Early Interactions between South and Southeast Asia: Reflections on Cross-Cultural Exchange, P.-Y. Manguin, A. Mani, et G. Wade (éd.), Singapore, New Delhi : Institute of Southeast Asian Studies, Manohar, 2011, p. XIII-XXXI. 
Recrutée par Paul Lévy dans une École française d'Extrême-Orient qui vivait dans l'immédiat après-guerre ses dernières années en Indochine, une nouvelle génération de chercheurs s'est déjà inscrite dans cette rupture, à laquelle appartiennent Maurice Durand, spécialiste de la culture vietnamienne, ou Louis-Charles Damais, épigraphiste féru de vieux javanais, pour n'en citer que deux ${ }^{11}$. Si ces chercheurs restent formés à l'indianisme ou à la sinologie, ils sont libérés des dominantes idéologiques de la période qui s'achève. Ils modifient dès lors sensiblement le regard qui est porté sur l'Asie du Sud-Est et sur son passé, comme l'avait réclamé brutalement Émile Gaspardone, dans son célèbre article de la Revue de Paris de 1936, en reprochant aux travaux de l'EFEO, et en particulier à l'archéologie indochinoise, d'avoir été absorbée par l'Inde, laissant à l'abandon les civilisations qui n'ont pas produit les grands monuments des États hindouisés. Passionné par le Vietnam, Gaspardone s’opposait ainsi déjà, implicitement, en quittant l'EFEO, au point de vue dominant des savants coloniaux d'Indochine comme des Indes néerlandaises, qui minimisaient, voire censuraient, les développements culturels endogènes. Il en allait ainsi des cultures préhistoriques du Vietnam qui ne pouvaient être qu'exogènes, ou encore des grands royaumes historiques et de leurs monuments, inconcevables sans l'intervention exclusive d'un deus ex machina chinois ou indien. Cette nouvelle génération de chercheurs de l'EFEO commençait donc à dégager l'étude de la culture et de l'histoire des pays de la région de l'emprise exclusive des deux grandes masses que sont la Chine et l'Inde, dont le voisinage imposant fit qu'on qualifia longtemps l'Asie du Sud-Est de termes révélateurs tels Insulinde, Indo-chine, Inde extérieure ou encore, chez les nationalistes indiens, Greater India, la Grande Inde (leur permettant de s'attribuer ainsi une mission civilisatrice passée, alors qu'ils se trouvent eux-mêmes colonisés). On a dès lors en effet pris conscience que, pour être des lieux privilégiés d'échanges avec les civilisations indiennes et chinoises, les pays de l'Asie du Sud-Est n'en ont pas moins formé des cultures régionales ou nationales spécifiques: les faits de civilisation que l'on y observe ne sont pas réductibles aux seuls phénomènes d'indianisation, de sinisation (et plus tard d'islamisation) d'un substrat primitif.

De fait, en ce qui concerne la préhistoire et la protohistoire du Vietnam, il faudra attendre que les archéologues vietnamiens, dans une

11. Cette génération ne pourra mener à terme son parcours: par une coïncidence fâcheuse, plusieurs d'entre eux décèderont dans les années 1960, encore jeunes, peu d'années après leur retour en France et leur intégration à l'École pratique des hautes études. 
approche tout à la fois appréciative et critique des travaux de la période coloniale, commencent à fouiller leur propre territoire, l'indépendance gagnée, pour que la démonstration soit faite que les cultures mises au jour par les chercheurs de l'école sont largement le produit de développements locaux et donc, dans une perspective politique, peuvent être constitutifs de l'identité nationale (ce qui ne va pas non plus sans l'introduction de biais considérables dans les interprétations nouvellement fournies...). Les rapports entre le premier royaume vietnamien - connu sous le nom de Văn Lang dans les sources écrites et longtemps considéré comme légendaire -, et la splendide culture de l'âge du bronze, dite de Đông Sơn, ont ainsi fait l'objet de révisions radicales ${ }^{12}$.

Sur le territoire vietnamien, ce sont bien plus les cultures hindouisées du Champa, dans le centre du pays, et plus au Sud celle du Funan (dites aujourd'hui par les Vietnamiens : culture de Oc Eo), qui feront dès les premières années de l'école l'objet de très nombreuses publications en épigraphie, comme en histoire de l'art et de l'architecture. L'étude des langues des minorités ethniques des hautes terres sera de même privilégiée, alors même que la modernisation de la romanisation du quoc $n g u$, pourtant inscrite dans la première lettre de route de l'EFEO, ne sera jamais abordée.

De même, si l'épigraphie des pays indianisés d'Asie du Sud-Est a fait l'objet dès avant même la création de l'EFEO de nombreux travaux, l'épigraphie sino-vietnamienne n'a pas fait l'objet d'autant d'attentions, tant s'en faut. L'histoire du Vietnam, telle qu'elle était perçue par les sinologues de l'EFEO, était avant tout celle d'une province réfractaire des marches de la Chine. Les inscriptions, qui ne fournissent pour l'essentiel que des informations très locales, n'avaient pour eux qu'un intérêt limité. Elles sont aujourd'hui l'un des outils essentiels d'une historiographie vietnamienne dégagée de l'ombre de son encombrant voisin Han. Quelque vingt mille estampages seront néanmoins rassemblés à la bibliothèque de l'école à Hanoi et légués aux chercheurs vietnamiens à l'indépendance de leur pays, montrant par là que l'école, en ce qui concerne le Vietnam, a été consciencieuse dans sa mission de conservation du patrimoine, mais ne l'a pas que tardivement et donc peu exploité.

12. Sur cette polémique, qui fera long feu à l'EFEO même, voir supra note 8 . Haydon Cherry a récemment approfondi la question de la construction de l'identité nationale vietnamienne, en réaction aux interprétations archéologiques de la période coloniale dans son « Digging up the Past: Prehistory and the Weight of the Present in Vietnam », Journal of Vietnamese Studies, 4 (1), 2009, p. 84-144. 
Seules la collecte et l'étude des sources textuelles sino-vietnamiennes sont de fait privilégiées à l'EFEO, grâce auxquelles sont jetées très tôt les bases d'une vietnamologie moderne. Acteurs d'un orientalisme pour lequel la civilisation chinoise, comme celle de l'Inde, était prééminente, les membres sinologues de l'école n'ont cependant que partiellement succombé à cette pensée unique et se sont donné les moyens d'étudier le Vietnam pour lui-même. Paul Pelliot, Henri Maspero et Léonard Aurousseau, chacun dans sa spécialité, ont apporté leur contribution à la connaissance du passé vietnamien, ouvrant en particulier la voie aux contributions majeures d'Émile Gaspardone dans les années 1930. On a vu plus haut combien ce dernier déplorait le peu d'intérêt relatif des chercheurs de l'école pour le Vietnam, au point de quitter l'EFEO avec fracas. Il a ainsi consacré ses années à l'EFEO à la philologie sino-vietnamienne, publiant entre autres quelques travaux épigraphiques et une fondamentale "Bibliographie annamite " (dans le BEFEO de 1934). Sinologue de formation, c'est le vietnamien qu'il choisit d'ailleurs d'enseigner à son retour en France, jusqu'à la guerre, à l'école des langues orientales.

Ces études sont accompagnées par celles de collaborateurs extérieurs, mais proches de l'école. Le R.P. Léopold Cadière, homme de grande culture, spécialiste du Vietnam, qu'aucun aspect de la civilisation vietnamienne ne laisse indifférent, publie à l'EFEO ses travaux plus philologiques. Charles Maybon fait un bref passage à l'école, qu'il quitte dès 1911 pour rejoindre le corps enseignant dont il est issu; il est l'auteur d'une remarquable thèse sur les rapports des Européens avec le Vietnam. L'école, dans les années 1930, accueille aussi dans ses collections les publications de géographes de renom, Charles Robequain et Pierre Gourou, d'une géologue et préhistorienne de génie, Madeleine Colani, ou encore d'un maître incontesté de la linguistique de l'Asie du Sud-Est, AndréGeorges Haudricourt.

Alors que le Vietnam et les autres peuples de l'Asie du Sud-Est se préparent à émerger en tant que nations modernes, la perception qu'on a de leur culture se modifie nettement et la nouvelle génération de chercheurs se spécialise d'emblée dans l'étude des diverses aires culturelles de l'Asie du Sud-Est. Parmi ceux-ci, Maurice Durand a mené les études vietnamiennes à leur maturité, en y mettant sa connaissance du chinois au service d'une vietnamologie autonome; ses connaissances sont polymorphes: grammairien, philologue, historien des religions et des techniques religieuses, historien de l'art, spécialiste de la littérature enfin, il a laissé une œuvre bien connue des vietnamologues. Il a contribué ainsi à dégager définitivement ces recherches du seul domaine de la sinologie, 
à une époque où le Vietnam restait encore communément perçu comme une turbulente dépendance de la Chine et ses traits distinctifs comme autant d'infidélités au grand modèle voisin. Le succès de l'entreprise a été consommé lorsqu'on a créé à l'EPHE pour Maurice Durand une première chaire d'études vietnamiennes ${ }^{13}$.

L'histoire de l'art et la conservation du patrimoine architectural vietnamien n'ont pas attiré l'attention de l'école autant que la statuaire ou les monuments indianisés du Champa (sur le territoire vietnamien) ou du Cambodge voisin. L'EFEO prête néanmoins dès 1918 son concours à l'administration locale pour la restauration du temple de la Littérature de Hanoi, où les mandarins célèbrent alors encore les rituels du culte à Confucius, et elle restaure en 1922 la pagode du Pilier unique de Hanoi, ainsi que de nombreuses pagodes endommagées par le typhon de 1929, puis les grandes pagodes de Bắc Ninh. Il faudra cependant attendre 1935 pour que soit engagé un architecte spécialisé, Louis Bezacier, pour occuper le poste de conservateur des monuments du Tonkin.

Le legs de l'EFEO au Vietnam le plus directement visible, aujourd'hui encore, pour un grand public est celui de la muséographie. La création de musées est inscrite dans sa charte de fondation. Dans sa lettre de mission au premier directeur de l'EFEO, Auguste Barth lui trace un programme précis:

Vous créez [...] un musée vraiment digne du nom, formé non pas au hasard, pour l'apparat et pour la curiosité, mais avec méthode et en vue du travail. On ne verra plus disperser dans les résidences ou envoyer au musée Guimet des morceaux dépareillés et, partant, sans valeur: l'IndoChine conservera ses richesses ${ }^{14}$.

Cette injonction à conserver en Indochine toutes les pièces archéologiques n'est pas parfaitement respectée: certaines pièces de moindre importance, déclassées, seront données à des musées d'Europe (ou du Japon, sous le régime de Vichy) ou vendues par les conservations du Cambodge ou d'Annam pour financer les travaux de restauration, surtout dans les années de crise. Les vives polémiques qui surgissent dans la presse locale ou au sein même de l'EFEO feront cesser ces exportations en $1945^{15}$.

13. On se reportera pour plus de détails aux nombreux passages consacrés aux études vietnamiennes dans C. Clémentin-Ojha et P.-Y. Manguin, Un siècle... ou encore à Philippe Le Failler, l'École française d'Extrême-Orient au Vietnam, 1900-2000: Regards croisés sur un siècle de curiosité scientifique, Ha Nôi, EFEO, 2000.

14. Cité dans C. Clémentin-Ojha et P.-Y. Manguin, Un siècle..., p. 18.

15. Sur ces dérives, voir C. Clémentin-Ojha et P.-Y. Manguin, Un siècle..., p. 82-83. 
Ce sont en fin de compte cinq grands musées qui sont créés au Vietnam, avec des statuts différents: les deux premiers appartiennent en propre à l'EFEO, les trois autres sont placés sous le seul contrôle scientifique de l'école. Le musée Louis-Finot à Hanoi, inauguré en 1933, est destiné à abriter en priorité les arts chinois, japonais et vietnamien, mais il a vocation aussi à devenir la vitrine de l'Indochine tout entière; il deviendra le Musée national d'histoire du Vietnam après l'indépendance. Dans un palais de l'enceinte royale de Huê, l'art vietnamien trouve aussi sa place au musée Khải Định, construit sous le haut patronage de l'empereur. En 1936, une ordonnance royale de l'empereur Bảo Đại crée le musée archéologique du Thanh Hóa, province richissime en vestiges de l'âge du bronze vietnamien. Le Musée de la sculpture cham de Đà Nẵng, comme son nom l'indique, est exclusivement consacré à l'art du Champa, dès sa fondation en 1918 sous le nom de musée Henri-Parmentier. Enfin, à Saigon, c'est le gouverneur Blanchard de la Brosse qui s'emploie à faire construire en 1929 le vaste musée qui portera longtemps son nom. Son fonds est au départ constitué par une collection privée en provenance de toute l'Asie et par les pièces laissées sous la responsabilité de l'EFEO lors de son installation à Hanoi. Celle-ci continue d'alimenter régulièrement les collections du musée de Saigon, en y abritant en particulier les pièces recueillies sur les sites archéologiques de Cochinchine jusqu'en 1945, dont la riche collection d'objets en provenance des sites du Funan rassemblée par son conservateur de l'époque Louis Malleret. Il porte aujourd'hui le nom de Musée d'histoire de Hô Chi Minh-Ville ${ }^{16}$.

Pour parachever ce survol rapide du legs de l'EFEO au Vietnam, il nous faut mentionner le fait que les collections chinoises et vietnamiennes de la bibliothèque attachée à son siège à Hanoi restent la propriété du Vietnam nouvellement indépendant, dans le cadre de l'application des accords de Genève. Dès 1931, selon les mots d'Alfred Foucher, elle était devenue « la mieux outillée du monde pour l'étude de l'ExtrêmeOrient ". Son fonds sino-vietnamien est unique; son fonds chinois, l'un des plus riches du monde; en 1956, réinstallée dans un immeuble de cinq étages spécialement construit à son intention, elle compte avec ses 47000 volumes en chinois, en nôm et en japonais, parmi les plus belles collections orientalistes du monde. On y trouve enfin quelque 22500 estampages d'inscriptions, et elle compte aussi plus de 2000 cartes et plus de

16. Voir pour plus de détails sur l'histoire de ces musées C. Clémentin-Ojha et P.-Y. Manguin, op. cit., p. 213-222. 
70000 photos. Elle sera attribuée à la réunification du Vietnam à l'Institut d'information des sciences sociales ${ }^{17}$.

\section{La dette de l'EFEO: les lettrés de Hanoi}

L'École française d'Extrême-Orient, au fil de ses années de présence à Hanoi, s'est attaché la collaboration d'un nombre conséquent de Vietnamiens grands connaisseurs de leur propre culture et forts, pour nombre d'entre eux, d'un recrutement par la voie classique des concours de mandarinat. Dès lors que ces derniers sont supprimés en 1918, l'ancienne classe des lettrés, socialement dévalorisée par les changements politiques et privée de tout pouvoir réel, entre ainsi au service de l'orientalisme. Ces travailleurs de l'ombre, qui ont longtemps œuvré sous la direction des philologues français à l'immense travail de collecte et de dépouillement des littératures sino-vietnamienne et vietnamienne, ont indéniablement joué un rôle essentiel dans les transferts de connaissances accomplis en un demi-siècle.

Sans leur constante collaboration, l'école n'aurait pu mener à bien les nombreuses études publiées par ses membres sur le Vietnam. Leurs noms apparaissent rarement dans les ouvrages de l'école, si ce n'est parfois pour des tâches subalternes, à l'image du statut qui leur est longtemps réservé par l'administration coloniale. Ce n'est qu'à la veille de la Seconde Guerre mondiale qu'un décret du président de la République française autorise l'EFEO à recruter des Indochinois dans le cadre de son personnel scientifique. Libéralisation à valeur surtout symbolique, puisque les conditions qui leur sont faites restent bien celles qui prévalent dans le reste du contexte colonial: ils reçoivent des émoluments se montant aux dix dix-septièmes de ceux perçus par «les fonctionnaires français d'origine, de même grade ".

Nombre d'entre eux jouent dans les milieux intellectuels vietnamiens de l'époque des rôles marquants. Ainsi, le précieux index systématique des vingt premiers volumes du Bulletin de l'École française d'Extrême-Orient a-t-il été signé par Nguyễn Văn Tố, le plus actif de ces collaborateurs, qui a publié aussi au Bulletin les notices nécrologiques de plusieurs membres correspondants de l'école spécialistes du Vietnam. L’indépendance acquise, il deviendra président de la chambre des députés de la République démocratique du Vietnam et ministre de l'Entraide

17. Sur la bibliothèque de Hanoi et son histoire, voir C. Clémentin-Ojha et P.-Y. Manguin, Un siècle..., p. 223-225. 
sociale. L'école a aussi accueilli dans ses rangs, entre 1908 et 1917, le lettré Pham Quynh, qui l'a quittée pour devenir le rédacteur en chef de la célèbre revue littéraire Nam Phong, où il défendra ses thèses sur une nécessaire collaboration avec la puissance coloniale et occupera des fonctions ministérielles dans le gouvernement de l'empereur Bảo Đại.

Paul Lévy, directeur de l'école entre 1947 et 1950, dont on sait les opinions anticolonialistes, met en pratique, sous une forme nouvelle, les recommandations qui avaient été faites à l'école dès sa création: faire participer à ses activités les peuples de l'Indochine. Il fait paraître à Hanoi, sous la bannière de l'EFEO, la revue bilingue Dân Việt Nam/Le peuple vietnamien, dont trois numéros sortiront jusqu'en 1949, avec la collaboration active de Trần Hàm Tấn, spécialiste de médecine traditionnelle, pilier depuis 1920 du Bureau des lettrés de l'école à Hanoi. Plus de la moitié des contributions de Dân Việt Nam sont signées de lettrés et d'érudits vietnamiens. Elle permet de faire connaître des études scientifiques de grande valeur sur les techniques, les traditions, le langage de la population vietnamienne.

Ceux d'entre ces collaborateurs qui ont su se former aux exigences méthodologiques des philologues ou des ethnologues français ont joué pour leur part un rôle inverse dans la transmission des savoirs, et servi de passeurs lors de l'accession du Vietnam à l'indépendance, étant mieux à même d'intégrer dans leur démarche intellectuelle une pluralité de références intellectuelles et politiques. Ils ont signé quelques contributions importantes de l'école aux études vietnamiennes. Parmi ceux-ci, nous n'en citerons que deux, faute de place ${ }^{18}$.

L’assistant Trần Văn Giáp a séjourné ainsi quelques années à Paris comme répétiteur de vietnamien à l'école des Langues orientales, et soutenu à l'EPHE un mémoire sous la direction de Paul Pelliot en 1931. De retour à Hanoi, il a assuré la gestion des fonds chinois et vietnamien de la bibliothèque de l'école et suivi l'impression de l'Inventaire $d u$ fonds chinois. Il a surtout publié en 1932 au Bulletin de l'EFEO un article fondamental sur "Le bouddhisme en Annam des origines au XIII siècle ». Entré au Parti communiste vietnamien en 1945, il rejoindra la résistance dès 1946. Il participera activement dans les années 1950 aux négociations qui mèneront au départ ordonné de l'école de Hanoi. Il prendra alors en charge la gestion du fonds documentaire transféré au gouvernement

18. Ces passages sur les lettrés vietnamiens de l'EFEO sont tirés pour l'essentiel du petit ouvrage sur l'EFEO au Vietnam publiée par son centre de Hanoi (Le Failler, l'École française d'Extrême-Orient au Vietnam...) et de C. Clémentin-Ojha et P.-Y. Manguin, Un siècle..., p. 144-147. On s'y reportera pour plus de détails. 
vietnamien et sera l'un des fondateurs du Comité d'étude sur la littérature, l'histoire et la géographie, ancêtre de l'actuel Institut d'histoire de Hanoi.

Nguyễn Văn Huyên obtint pour sa part en 1929 à la Sorbonne une licence en histoire-géographie et y devint en 1934 le premier docteur ès lettres vietnamien, avec une thèse en ethnologie. Il a enseigné un temps à l'École nationale des langues orientales et comptait déjà de nombreuses publications à son actif quand il devint en 1939 le premier membre scientifique vietnamien de l'EFEO ${ }^{19}$. Il sera ministre de l'Éducation de la République démocratique du Vietnam.

$$
* * *
$$

Après ce trop bref survol des conditions dans lesquelles l'École française d'Extrême-Orient a exercé ses multiples activités scientifiques et administratives dans le cadre de l'Indochine française, que conclure en ce qui concerne les savoirs qu'elle avait pour mission, d'abord, de construire, mais aussi de transmettre?

À qui ces recherches ont-elles profité: à la science, ou bien à l'administration coloniale qui les finançait? En cette première moitié du xx siècle, et dans le cadre de la mission et du statut ambigus assignés à l'école, poser ainsi cette question en termes mutuellement exclusifs constitue un anachronisme. Dans un contexte historique complexe, en prenant pour objet d'étude des sociétés dépendant en Indochine d'administrateurs coloniaux, le personnel scientifique de l'EFEO semble bien avoir eu une nette conscience de ses devoirs envers les premières comme envers les seconds. Pour reprendre les mots de Paul Rivet au premier congrès des préhistoriens de Hanoi en 1932, tels que retranscrits par l'ethnologue et préhistorien Paul Lévy:

Car s'il est, en effet, important du point de vue scientifique d'avoir sur les populations anciennes et actuelles de l'Indochine les archives les plus complètes possibles, il n'est pas moins essentiel, sans parler du rang que la Science indochinoise est politiquement tenue de garder aussi bien en Extrême-Orient qu'ailleurs, pour les dirigeants de connaître à fond, c'està-dire scientifiquement, la vie passée et actuelle des peuples dont le futur leur a été confié.

19. Les chants alternés des garçons et des filles en Annam, Paris, P. Geuthner, 1934 ; Introduction à l'étude de l'habitation sur pilotis dans I'Asie du Sud-Est, Paris, P. Geuthner, 1934. 
Bien plus tard, en 1947, alors qu'il est devenu directeur de l'EFEO et que s'annonce la guerre de libération, le même Paul Lévy, dont on a déjà vu qu'il ne pouvait être soupçonné de biais colonialistes, est resté fidèle à ce point de vue, exprimé lors d'une causerie sur les ondes de Radio-Saigon:

Est-il besoin, maintenant, d'insister sur la valeur sociale, politique, matérielle même de ces travaux? Des esprits non avertis de la valeur de la Science ont pu négliger les moyens de connaissance, donc d'action, que l'École française d'Extrême-Orient mettait ainsi à la portée des dirigeants; je ne pense pas que de bons résultats ne soient jamais venus couronner leurs œuvres. Seule l'étude des problèmes humains telle que nous pouvons la faire, c'est-à-dire la plus complète, la plus impartiale, la plus désintéressée, seule une pareille étude pourra permettre des solutions durables aux problèmes de l'heure présente ${ }^{20}$.

20. Nous avons tiré les deux citations de Paul Lévy et la teneur de ces passages, donnés ici en guise de conclusion générale, de C. Clémentin-Ojha et P.-Y. Manguin, Un siècle... (p. 182-183), où ils étaient discutés dans le seul cadre des développements de la discipline ethnologique en Indochine. 


\section{Note bibliographique}

Pour une brève histoire de l'EFEO, voir C. Clémentin-Ojha et P.-Y. Manguin, Un siècle pour l'Asie: l'École française d'Extrême-Orient 1898-2000, Paris, EFEO, Les Éditions du Pacifique, 2001. Sauf indication contraire, les données chronologiques qui sous-tendent cet article en proviennent. Mais ce petit livre n'est pas fondé sur des recherches approfondies dans les archives de l'EFEO (aujourd'hui encore en cours de classement) ou du gouvernement colonial; une véritable histoire de cette institution reste donc à écrire. En dehors des textes publiés au fil des ans par l'école elle-même, il existe néanmoins quelques ouvrages où l'histoire indochinoise de l'EFEO apparait de façon récurrente, mais qui souffrent tous de n'avoir pas utilisé des fonds d'archives plus complets. Le remarquable travail de Penny Edwards est consacré au seul Cambodge, autre nation de l'Indochine française où l'EFEO, bien plus qu'au Vietnam, a joué un rôle de prescripteur dans les domaines culturels, via l'Institut d'études bouddhiques comme de par son rôle de révélateur de la grandeur angkorienne (Cambodge: the cultivation of a nation, 18601945. Bangkok, Silkworm, 2008). On se reportera aussi au travail de Pierre Singaravélou (L'École française d'Extrême-Orient, ou l'institution des marges, 1898-1956, Paris, L'Harmattan, 1999), une étude essentiellement prosopographique de l'histoire de l'EFEO; à l'ouvrage de Laurent Dartigues (L'Orientalisme français en pays d'Annam 1862-1939: essai sur l'idée française du Viêt Nam. Paris, Les Indes Savantes, 2005); à l'article de Jean-François Klein, "L'histoire de l'Indochine en situation coloniale. Entre histoire et orientalisme (1858-1959) ", in La construction du discours colonial: l'empire français aux $X I x^{e}$ et $x x^{e}$ siècles, Oissila Saaïdia and Laurick Zerbini (éd.), Paris, Karthala, 2009, p. 89-124; ou encore aux études d'Amaury Lorin consacrées au rôle joué par Paul Doumer dans la création des institutions d'enseignement supérieur en Indochine française (Paul Doumer, gouverneur général de l'Indochine (1897-1902), Paris, L'Harmattan, 2004; «L'École française d'Extrême-Orient, création de Paul Doumer, gouverneur général de l'Indochine (1898): un acte politique ", in Les relations culturelles internationales au $x x^{e}$ siècle: de la diplomatie culturelle à l'acculturation, Anne Dulphy, Robert Frank, Marie-Anne Matard-Bonucci, et al. (éd.), Bruxelles: P.I.E. Peter Lang, 2010, p. 339-345). 


\section{Pratiquer la médecine enseignée à la française}

\section{L'accès des médecins vietnamiens à l'exercice libéral et ses enjeux dans l'entre-deux-guerres} Laurence Monnais

En mai 1929, le Dr Nguyễn Văn Luyện (1898-1946) fait paraître dans le magazine de santé hanoien Vệ sinh báo. Journal de vulgarisation d'hygiène, un article intitulé " La médecine occidentale et la nôtre " [Thuốc tây và thuốc ta]. On y lit:

[...] La médecine occidentale utilise beaucoup de remèdes que l'on retrouve dans notre propre pharmacopée [...] Nos scientifiques doivent se concentrer sur l'analyse de l'efficacité de nos remèdes selon la méthode expérimentale occidentale [...]; l'expérience servira de base et les tests comme preuve pour pratiquer cette médecine. L'utilisation des remèdes occidentaux augmente de jour en jour mais on ne peut mettre de côté les remèdes dont nous avons hérité [NB : entre autres parce qu'ils sont adaptés aux constitutions vietnamiennes, précise plus loin l'article] [...] ${ }^{21}$.

Ces mots sont inattendus et quelque peu transgressifs eu égard au double fait que Luyện vient de soutenir son doctorat en médecine à Paris (1928) et qu'il s'apprête à s’installer à son compte à Hanoi (janvier 1931): à l'époque, la médecine vietnamienne traditionnelle est absente, voire

21. Nguyễn Văn Luyện, "Thuốc tây và thuốc ta " ("Les médicaments occidentaux et les nôtres »), Vệ sinh báo, n 40, mai 1929, p. 3-7. 
mise au ban de l'effort de médicalisation du Vietnam. Simple étrangeté de surface? À déconstruire les propos de ce professionnel de la santé, on se rendra compte qu'ils attestent l'existence d'un double mouvement, d'incorporation du modèle biomédical auquel les médecins colonisés ont été formés, mais aussi d'adaptation en retour de cette médicalisation à la française à laquelle ils devaient allégeance et dont ils étaient destinés à devenir les héritiers naturels. Exception ordinaire, pour reprendre François Dosse ${ }^{22}$, la carrière de Luyện - avec celle, en filigrane, de quelques-uns de ses collègues - permet d'illustrer certaines caractéristiques d'un groupe négligé dans les histoires de la santé tant coloniales que vietnamiennes; un groupe pourtant révélateur de transferts culturels complexes qui ont à voir avec leur statut multiple de scientifique, de professionnel, de colonisé et de thérapeute bien sûr, mais d'abord et avant tout de médiateurs culturels: celui des médecins vietnamiens ayant exercé la médecine en libéral avant 1945.

\section{Un bref portrait quantitatif et qualitatif}

On tient généralement pour acquis que la prise en charge de la santé dans les colonies françaises fut l'œuvre de médecins blancs, fonctionnaires et militaires, ayant œuvré dans le cadre de systèmes publics de santé, telle l'Assistance médicale indigène (AMI). Des médecins orientés vers la prévention collective des maladies tropicales les plus meurtrières et assistés dans cette mission par quelques poignées de praticiens indigènes mal considérés et exploités ${ }^{23}$.

Ce tableau mérite en fait quelques retouches, on s'en rend compte si l'on se met à explorer des sources alternatives aux sources coloniales habituelles. Au-delà de mémoires et d'archives privées qui n'ont que trop rarement survécu, il s'agit d'abord et surtout de la presse populaire de l'époque, foisonnante, bilingue (en français et en quốc ngữ) et diverse, qui nous informe de multiples façons sur ces médecins (articles de fond dont ils sont auteurs ou protagonistes, mention des honneurs reçus ou d'une nouvelle affectation, encarts publicitaires pour leur cabinet ou clinique) mais aussi des listes de patentés, voire d'abonnés au téléphone à partir de la fin des années 1930. Grâce à ces sources variées, ce sont

22. François Dosse, Le pari biographique. Écrire une vie, Paris, La Découverte, 2005.

23. Nous avons assurément participé à façonner cette historiographie, coincée, comme les autres, par des sources disparates et discrètes sur ces subalternes et leurs activités. Sur I'AMI et son histoire indochinoise voir : Laurence Monnais-Rousselot, Médecine et colonisation. L'aventure indochinoise, 1860-1939, Paris, CNRS Editions (« Histoire »), 1999. 
en fait 576 diplômés en médecine d'origine vietnamienne que l'on a pu recenser pour la période 1905-1940, ce qui représente environ la moitié des médecins ayant travaillé en Indochine à un moment de leur carrière, toutes origines ethniques confondues. Plus d'un quart de ces praticiens (27\%, contre $10 \%$ des médecins français) et ce chiffre est probablement inférieur à la réalité ${ }^{24}$ auraient par ailleurs, à un moment donné de leur carrière, choisi la voie de l'exercice libéral, échappant ainsi au carcan de l'Assistance. Entre 70 et 75 \% si l'on se concentre sur les années 1930.

\section{L'école de médecine de Hanoi et ses meilleurs étudiants}

Pour comprendre les raisons d'être de ce mouvement, avant de s'interroger sur ses implications concernant l'espace vietnamien de la santé, il faut revenir sur les conditions de formation de ces médecins. Ils sont en effet a priori tous issus de l'école de médecine de Hanoi, qui a ouvert ses portes en 1902, élément pionnier dans la fondation d'une Université indochinoise $\mathrm{e}^{25}$.

L'objectif est clair en 1902: former des auxiliaires indigènes, des techniciens médicaux qui œuvreront à la médicalisation de l'Indochine sous la houlette des médecins français et sauront, comme il se doit, défaire l'influence des charlatans, autrement dit des thérapeutes chinois et vietnamiens dont les colonisateurs n'avaient pas manqué de mesurer l'influence sur la société ${ }^{26}$. En 1904, le pastorien et découvreur du bacille de la peste Alexandre Yersin, premier directeur de l'école (apparemment piètre administrateur, il démissionnera d'ailleurs assez vite) énumère au

24. Les sources dont on dispose sur ces médecins sont à la fois hétérogènes, dispersées et très fragmentaires. De ce fait, il n'est pas toujours possible de savoir si un médecin a exercé à son compte ou nom à un moment de sa carrière.

25. Pour plus de détails sur l'histoire de l'école de médecine de Hanoi voir : Laurence MonnaisRousselot, "La professionnalisation du "médecin indochinois" au XXe siècle: Des paradoxes d'une médicalisation coloniale», Actes de la recherche en sciences sociales, $n^{\circ} 143,2002$, p. 36-43.

26. Nombreux sont les médecins coloniaux qui, dès les années 1860 et la domination de la Cochinchine, critiquent vertement l'empirisme de ces thérapeutes et la naïveté des malades qui y ont pourtant recours en masse. À l'heure d'une médecine occidentale qui revendique sa supériorité scientifique et de ce fait l'exclusivité, surtout dans le cadre colonial civilisateur, il n'était pas question de laisser se perpétuer cette concurrence (Laurence Monnais, "Traditional, Complementary and Perhaps Scientific? Professional Views of Vietnamese Medicine in the Age of French Colonialism, » Southern Medicine for Southern People: Vietnamese Medicine in the Making, Laurence Monnais, C-Michele Thompson et Ayo Wahlberg (éd.), Newcastle upon Tyne, Cambridge Scholars Publishing, 2012, p. 61-84). 
résident supérieur du Tonkin les compétences dont pourront se prévaloir les jeunes diplômés: formés sur trois ans (six en métropole dont l'année préparatoire post-baccalauréat PCN avec formation en physique, chimie, sciences naturelles ${ }^{27}$ ), ils sauront examiner un malade, diagnostiquer les maladies courantes, prescrire et préparer les médicaments usuels (leur droit de prescription restera toutefois longtemps limité); ils maîtriseront l'art du pansement et celui de la vaccination et, aptes à procéder à quelques opérations (ouverture d'abcès, panaris, sondage de la vessie), seront de "précieux auxiliaires du chirurgien français, sachant administrer le chloroforme et manier les instruments ${ }^{28}$. Dans les faits, ces auxiliaires tinrent lieu de chargés de propagande en faveur des bienfaits civilisateurs de la prévention collective, par la vaccination entre autres, et leur rôle fut celui de courroie de transmission entre la médecine occidentale si supérieure et la population colonisée.

À l'annonce de son ouverture, l'école aurait reçu 375 dossiers de demandes de jeunes hommes, du Nord comme du Sud; trente devaient être retenus, sur la base de leurs motivations et de leurs performances scolaires - quoique tous n'aient pas achevé le cycle secondaire et qu'un certain nombre n'eût pas la maîtrise du français, ce qui obligera l'école à engager des interprètes. La première cohorte, de cinq diplômés seulement, sort en 1905 à l'heure, ce n'est évidemment pas un hasard, de la formalisation de l'AMI. Ils ont donc le titre de médecins auxiliaires indigènes et ne peuvent pratiquer que la médecine indigène; autrement dit, ils ne sont habilités à prodiguer leurs soins qu'aux colonisés, gratuitement qui plus est, dans le cadre fonctionnarisé de l'Assistance. Affectés aux quatre coins de la péninsule indochinoise, ils doivent accomplir deux ans de stage avant d'être titularisés... et mutés tous les deux ans en moyenne, voire moins. Quelques fils de bonne famille — issus du milieu mandarinal mais aussi de celui des affaires - partiront toutefois pour la France, une bourse du gouvernement colonial en poche, afin de poursuivre des études doctorales. Dès 1908, trois étudiants jugés particulièrement prometteurs sont ainsi envoyés à la faculté de médecine de Paris sur proposition du nouveau directeur de l'école de Hanoi, le Dr Cognacq. L'événement est significatif. Témoignant d'un fonctionnement $a$ priori méritocratique, il marque la possibilité pour les meilleurs étudiants de

27. Le certificat d'études physiques, chimiques et naturelles (dit PCN) deviendra à partir de 1934 certificat d'études physiques, chimiques et biologiques (dit PCB).

28. Archives nationales du Vietnam (ANVN), centre $n^{\circ} 1$ (Hanoi), Fonds de la résidence de $\mathrm{Ha}$ Dong, dossier 3767. 
sortir du carcan d'une profession technique. Reste que, sujets vietnamiens et non citoyens français, ces étudiants ne pourront obtenir qu'un doctorat universitaire et non un doctorat d'État, permettant seul d'exercer pleinement la médecine au regard de la loi médicale française de 1892, appliquée à l'Indochine ${ }^{29}$. "Leur place [sera toute] indiquée dans nos laboratoires [et] dans nos grands centres hospitaliers [...] » explique Cognacq; " mais [...] il ne saurait être question de leur donner une plus grande liberté d'action ni de les libérer de la surveillance d'un médecin européen [...] » précise-t-il.

\section{Expatriation, expertise et hiérarchies}

Cette immigration universitaire va augmenter de façon linéaire; dans les années 1920, l'on trouve des étudiants vietnamiens à la faculté de médecine de Paris, mais également à celles de Bordeaux, Montpellier, Lyon, Marseille, Toulouse. Certains arrivent même à convaincre l'administration hanoienne de leur permettre de rester en métropole après avoir soutenu leur thèse, afin de suivre un stage de spécialité ou de recherche. C'est le cas de Nguyễn Văn Thịnh (1888-1946). Comptant parmi les premiers boursiers du gouvernement en 1909, il est reçu avec les honneurs (deuxième) au diplôme de médecine coloniale de l'université de Paris l'année suivante, consécration universitaire qui pousse le ministre de l'Instruction publique à l'autoriser à s'inscrire en doctorat. Ses excellents résultats seront rapportés aux autorités de l'Indochine par le biais de lettres élogieuses de grands pontes parisiens ${ }^{30}$. En 1912, le voici externe des hôpitaux; alors qu'il accomplit son service à l'hôpital Saint-Antoine, il est accueilli par l'institut Pasteur et trouve le temps de collaborer à diverses revues spécialisées. Il ne rentrera au Vietnam pour servir l'AMI que pour quelques années: on le retrouve en effet de nouveau à Paris en 1919, avec la bénédiction du gouvernement général. Deux ans plus tard il soutient une thèse sur le béribéri sous la direction du grand spécialiste des maladies tropicales de l'époque, le Dr Jeanselme.

Avec les années 1930, cette immigration académique semble se tarir, mais cela s'explique par l'accession de l'école de Hanoi au rang de faculté,

29. Le doctorat d'état est en effet réservé aux citoyens français (les Vietnamiens naturalisés y ont donc accès mais ils sont extrêmement peu nombreux à l'époque et n'embrassent pas forcément la profession médicale...).

30. Centre des archives d'Outre-Mer (CAOM), Fonds des amiraux, dossier 2564, "Instruction publique. Groupe d'enseignement indochinois en France, Médecins indigènes envoyés en France à l'effet de perfectionner leur instruction médicale, 1897/1905/ 1916 ». 
en 1933. Dès la Première Guerre mondiale, l'administration coloniale a dû repenser la mission du médecin indigène - et par extension sa formation - alors que les effectifs médicaux en provenance de la métropole s'amenuisent; on a été forcé par conséquent de faire appel aux auxiliaires pour mener à bien des tâches qui n'étaient jusque-là pas les leurs: ils allaient devoir diriger des postes médicaux d'importance, prescrire des médicaments sans solliciter d'autorisation et par là même faire la preuve de leurs compétences, de leur polyvalence et de leur autonomie. Et c'est en fait dès 1919 que l'on ouvre une filière doctorale à Hanoi; le cursus est de six ans et le prérequis d'être titulaire d'un baccalauréat pour s'inscrire en année $\mathrm{PCN}^{31}$. Les étudiants de cette filière doivent encore faire leur thèse dans une université en France. Mais sur la base du triptyque métropolitain théorie-travaux pratiques-stages, on mise déjà sur le plus déploiement de l'éventail des disciplines enseignées (histologie, anatomopathologie, bactériologie); la variété de la formation clinique et scientifique proposée par les structures hospitalières alentour et les laboratoires de recherche de pointe affiliés déterminent autant qu'ils légitiment l'évolution institutionnelle de l'école. En 1926, est créé en annexe à cette dernière un institut médico-légal qui ouvre sur la conquête d'une nouvelle autonomie: celle d'agir en tant qu'expert auprès des tribunaux et de procéder à des autopsies. Le recrutement professoral s'opère quant à lui de plus en plus parmi les agrégés de l'école de médecine tropicale de Marseille, le Pharo, parmi les assistants des hôpitaux coloniaux métropolitains ou encore les pastoriens. Six nouvelles chaires d'enseignement magistral voient bientôt le jour dont une de parasitologie et une de médecine tropicale (1937). Hanoi fait finalement soutenir ses premiers doctorats en 1935. Entre-temps, en janvier 1936 plus précisément, un décret du ministère des Colonies amendait la loi de 1892 pour permettre aux sujets de tout l'Empire d'accéder au doctorat d'État.

Si le succès de ce programme doctoral est évident, il restera néanmoins terni jusqu'en 1939 par la coexistence de deux catégories de médecins vietnamiens, une hiérarchie abolie en France depuis plus de quarante ans avec la disparition des officiers de santé (1893). Plus mal vécu sans doute encore, les titulaires d'un doctorat (bác sĩ) sont assimilés à leurs confrères non docteurs ( $y$ sĩ), les uns étant comme les autres fonctionnaires de l'état colonial dans le cadre latéral de l'AMI réservé aux médecins indochinois, avec une même grille de traitement et d'avancement. Alors que les rangs

31. Le baccalauréat est désormais accessible via un certain nombre de lycées dans le cadre du système d'enseignement franco-indigène mis en place en 1917. 
de cette profession à deux vitesses grossissent de façon assez rapide, répondant à une volonté officielle de vietnamisation du personnel médical (dès 1921, il y a plus de médecins vietnamiens que de médecins français dans l'Assistance), il n'en reste pas moins que les titulaires d'un doctorat sont trop souvent maintenus à des postes subalternes, constamment mutés dans des régions rurales et insalubres dont leurs confrères français ne veulent pas et confrontés à des difficultés récurrentes d'avancement. Ce malgré leur expertise, une production scientifique à la pointe de leur domaine (qu'il s'agisse de la léprologie, de la nutrition, des déterminants sociaux de la santé ou de la mortalité infantile, sujet de thèse de Luyện) et un engagement sur le terrain fréquemment remarqué (par une médaille des épidémies ou de service à la vaccination). On peut imaginer les frustrations qui animaient alors certains et comprendre leur envie de quitter le fonctionnariat, pour mettre leur expertise au service d'une clientèle privée $^{32}$. Mais encore eût-il fallu qu'ils en aient le droit...

\section{De la sphère publique à la sphère privée}

La possibilité pour les médecins vietnamiens d'exercer en libéral ne s'est en effet concrétisée que très lentement. Une restriction voulue de la part des autorités coloniales: s'il était difficile de mettre en doute le bagage scientifique et la grande expérience du terrain pathologique indochinois de ces professionnels de la santé dès la fin du premier conflit mondial, on craignait à Hanoi que ces derniers ne veuillent s'installer qu'en ville, risquant d'y faire une concurrence déloyale aux médecins français (qui eux avaient le droit de faire du privé, qu'ils aient été fonctionnaires ou non) et hypothéquant la diffusion de soins essentiels en milieu rural, là où l'AMI les employait en priorité. Le fait que la très grande majorité d'entre eux n'ait qu'un doctorat universitaire (voire pas de doctorat du tout) permettait au gouverneur général de contrer assez facilement les demandes en ce sens, en se réfugiant derrière la loi. Une toute petite brèche s'était là encore ouverte à l'heure du premier conflit mondial - dans les conditions de pénurie que l'on a déjà évoquées quand en 1915, on autorisa les médecins indigènes à percevoir des honoraires de malades soignés hors des hôpitaux de l'AMI. Entre 1920 et 1931, plusieurs textes allaient formuler les modalités d'une autorisation plus franche mais qui resterait distribuée au compte-gouttes, nominative,

32. Plusieurs s'associeraient assez précocement pour revendiquer plus de droits et d'autonomie sur le terrain dont autour de l'Amicale des médecins auxiliaires dans les années 1920. 
donnée pour une localité prédéterminée et révocable; surtout il fallait avoir préalablement servi au moins dix ans dans le service public pour s'en prévaloir. Ce n'est finalement qu'en mars 1937 que l'autorisation on ne parle toujours pas de droit - était étendue à tout médecin diplômé de Hanoi.

\section{Un vent de liberté lucratif}

Quoi qu'il en soit, c'est bien avec les années 1930 que s'accomplit cette émancipation du joug fonctionnarial, au profit surtout des médecins passés par la France. Vers 1940, environ cent vingt praticiens évoluent en toute liberté, dont plus de quatre-vingt dans la seule colonie cochinchinoise, désormais rassemblés en un syndicat des médecins civils de l'Indochine. Si beaucoup sont passés par la France, y sĩ et bác sĩ vont coexister pendant un temps dans cet espace du soin privé. Mais à la veille de la Seconde Guerre mondiale, la grande majorité des médecins vietnamiens du privé est titulaire d'un doctorat d'état: selon un recensement effectué par les autorités coloniales, c'est le cas de $88 \%$ (50 sur 57) des médecins pratiquant en privé à Saigon en $1944^{33}$. Il faut dire que, à l'époque, la filière auxiliaire a disparu depuis plus d'une décennie déjà (1932), faute de candidats, et que la faculté de médecine monte son quotient de diplômés à soixante-quinze jeunes docteurs par an en 1938. Dès le début des années 1920, il n'était pas non plus rare de voir un y sĩ profiter d'un congé administratif pour retourner sur les bancs de l'école, à Hanoi ou à Paris, afin d'obtenir ce doctorat tant convoité, voire de se spécialiser et espérer ainsi disposer un jour d'une pratique lucrative. Au-delà de la liberté de mouvement (et de pratique) que la sortie de l'Assistance permettait, les médecins libéraux auraient en effet gagné entre deux et cinq fois plus que leurs collègues du cadre latéral.

Le parcours de Nguyễn Văn Luyện révèle cette progressive libéralisation de la profession médicale vietnamienne et l'évolution de sa stature professionnelle mais aussi probablement socio-économique. Originaire du Tonkin, Luyện entre à l'école de médecine de Hanoi en 1916 à l'âge de dix-huit ans (sans avoir terminé ses études secondaires); il en sort comme il se doit trois ans plus tard. Après ses deux ans de stage, il intègre officiellement l'AMI et est nommé à son premier poste, au Tonkin, en 1921.

33. ANVN, centre $n^{\circ} 2$ (Hô Chi Minh Ville), Tòa Đại Biểu Chính Phủ Nam Việt, S 09-6, « Liste nominative des docteurs en médecine diplômés d'état (européens et indochinois) exerçant leur profession à titre privé sur le territoire de Saigon-Cholon, 1944 ». 
En 1925, alors qu'il travaille à la léproserie de Huong Phong (Phu Tho, Tonkin), il demande au directeur de l'instruction publique de l'Indochine de pouvoir se faire créditer le baccalauréat dont il dit être désormais détenteur et l'examen PCN en fin de première année préparatoire qu'il vient de réussir afin de poursuivre ses études doctorales en France ${ }^{34}$. Il obtient gain de cause; dès l'année suivante il reçoit même une bourse d'un an, qui sera renouvelée l'année suivante, lui permettant de soutenir sa thèse à Paris en 1928. Thèse qui reçut les félicitations du jury et même les honneurs de l'Académie de médecine, annonce le quotidien hanoien Báo đông pháp le 24 avril $1928^{35}$. L'on ne connaît pas précisément les raisons qui vont finalement pousser le jeune docteur à quitter les rangs de l'AMI (on peut en soupçonner certaines); ce que l'on sait c'est que, revenu au Vietnam dès mai 1928 et envoyé le mois suivant à Hung Yen (au Tonkin toujours) en qualité de médecin indochinois de quatrième classe, il se mettait en disponibilité deux ans plus tard et... ouvrait un cabinet médical de médecine générale, pédiatrie et maladies vénériennes, 8 rue de la Citadelle à Hanoi ${ }^{36}$.

Concernant leurs émoluments, il faut bien comprendre aussi que l'intrusion dans l'espace de santé vietnamien de médecins exerçant en libéral ne put se faire qu'en réponse à l'émergence de ce que l'on peut appeler un espace colonisé du soin et d'abord d'un bassin critique de malades et de patients susceptibles de pouvoir payer de leur poche un médecin formé à l'occidentale: à l'heure où Luyện s'installe, il fallait en effet débourser entre deux et cinq piastres (\$) pour une consultation en cabinet (sans compter le coût associé à une ordonnance); à titre de comparaison, une journée d'hospitalisation dans une clinique privée atteignait en moyenne trois piastres et un traitement contre le rhume disponible en pharmacie (sans ordonnance cette fois), 0,5 \$ - rappelons que recourir aux services de l'Assistance restait gratuit pour les indigents, à savoir la grande majorité de la population. Cette privatisation des soins médicaux aurait par conséquent été circonscrite aux principaux centres urbains, à Hanoi (où l'on peut relever une trentaine de cabinets vers 1940) et surtout à Saigon-Cholon (près de soixante) là où le changement social passait par l'émergence de nouveaux groupes sociaux (dont des commerçants et des professions libérales), d'une classe moyenne diraient certains, en

34. CAOM, Fonds du gouvernement général (Gougal), dossier 51 402, « Dossier de Nguyễn Văn Luyện ».

35. Báo đông pháp, n503, 24 avril 1928.

36. Publicité, Vệ sinh báo, nº 61, janvier 1931. 
lien avec un dynamisme économique indéniable ${ }^{37}$; mouvement qui ne pouvait par ailleurs concerner qu'une minorité de la population citadine, éduquée et masculine ${ }^{38}$.

\section{À propos de l'offre et de la demande}

Pour que cette pratique privée puisse s’épanouir, il fallait, sinon qu’ait eu lieu une transformation des repères profanes en santé, du moins que le goût de la biomédecine venue avec le colonisateur et celui d'une relation thérapeutique à l'occidentale se soient développés. La question n'était pas seulement de pouvoir se payer un médecin, il fallait le vouloir, reconnaître dans le médecin vietnamien formé à la française un expert en santé digne de confiance et capable de répondre à des besoins précis, voire de guérir. Et cette acculturation était bien en cours dans l'entre-deux-guerres: on notera à cet égard l'importance donnée dans les encarts publicitaires, annonçant l'ouverture d'un cabinet ou vantant la réputation d'un praticien ayant déjà pignon sur rue, à l'énumération des diplômes obtenus (surtout s'ils l'ont été en France là encore), des stages effectués (à l'étranger mais aussi auprès d'institutions de recherche comme les instituts Pasteur d'Indochine) et même de la carrière, le cas échéant, menée au sein de l'AMI, gage évident de sérieux, de compétence et de polyvalence. La tradition confucéenne, historiquement forte au Vietnam, de valorisation des diplômes et de l'expérience ne saurait être la seule explication de ces tactiques promotionnelles. En 1933, le Dr Lê Văn Chinh, diplômé de la faculté de médecine de Toulouse (1920) se présente ainsi: "médecin qui a plus de trente ans de pratique en médecine; a été au Tonkin, en Annam, en Cochinchine; a fait cinq séjours en France et a été dans plusieurs hôpitaux " ${ }^{39}$. Le y sĩ Nguyễn Văn Khỏe, quant à lui, s'installe à Tra Vinh-ville en 1940 et évoque « vingt-six ans

37. Sur la notion de classe moyenne appliquée au Vietnam colonial voir : Van Nguyen-Marshall, Lisa B. Drummond et Danièle Bélanger (éd.), The Reinvention of Distinction. Modernity and the Middle Class in Urban Vietnam, Singapour, Springer-National University of Singapore Press (ARI -Springer Asia Series), 2012.

38. Toutes proportions gardées, il faut savoir que selon les estimations de l'historien George Weisz ( $R$ Regulating Specialties in France during the First Half of the Twentieth Century, » Social History of Medicine, vol. 15, n³, 2002, p. 463), il y avait près de 4000 médecins à Paris pour une population seulement deux fois plus importante que celle de Saigon en 1935. Par ailleurs il est clair que la concurrence pouvait être rude entre praticiens libéraux (ce qui expliquerait en partie la surenchère de publicités pour les cabinets de l'un ou l'autre dans les pages de plusieurs quotidiens de l'époque) et que si certains avaient rapidement joui d'une notoriété - et d'une position économique- enviables d'autres avaient du mal à fidéliser une clientèle.

39. Publicité, Le Progrès annamite, n 13, 20 juin 1933. 
de pratique " ajoutant qu'il est "[...] ex-préparateur de bactériologie à Phnom Penh; ex-médecin adjoint de chirurgie des hôpitaux mixtes de Phnom Penh et Lalung-Bonnaire de Cholon, ex-médecin adjoint au centre antituberculeux de Cochinchine [...] ex médecin-chef de Kompong Speu, Siem Reap, Stung Treng [...], Mallicole-Sud aux Nouvelles Hébrides [...] et Tra-vinh " ${ }^{40}$; on notera d'ailleurs qu'il s'est installé à son compte dans la région de sa dernière affectation publique ${ }^{41}$.

Autre preuve de cette acculturation: l'apparition de cabinets spécialisés, une tendance métropolitaine récente et toute biomédicale ${ }^{42}$. Si la présence (majoritaire) de spécialistes des maladies vénériennes, des dermatologues, gynéco-obstétriciens, pédiatres et ophtalmologues renvoie à une prise en charge de maladies prévalentes en Indochine ou de domaines de la santé qui sont l'objet d'anxiétés populaires incontestables (telle la santé reproductive), celle de cabinets à la pointe de technologies récentes (et de ce fait forcément coûteuses) offrant des traitements aux rayons $\mathrm{X}$ ou des séances d'électrothérapie manifestent une confiance de certains thérapeutes dans leur pouvoir d'attraction et, par extension, l'appropriation par une certaine clientèle de nouveaux repères thérapeutiques et diagnostiques. Luyện, pédiatre et spécialiste des maladies vénériennes, n’échappe d'ailleurs pas au mouvement proposant de l'électrothérapie à sa clinique attenante à son cabinet. La technique est alors utilisée à diverses fins thérapeutiques dont le traitement des hémorroïdes, de la blennorragie, de l'eczéma, de certaines douleurs neuromusculaires; associée à des rayons $\mathrm{X}$, elle sert aussi de physiothérapie.

\section{Défenseurs d'un pluralisme médical? Pour une médecine accessible à l'heure du droit à la santé}

Cette médicalisation à l'occidentale des villes vietnamiennes n'aurait toutefois pas exclu des entorses au modèle biomédical, entorses que vont

\footnotetext{
40. Publicité, L'Écho annamite, $n^{\circ} 183,19$ au 20 juin 1940.

41. Certains parmi ces médecins libéraux continueraient même d'ailleurs à œuvrer dans ou pour I'AMI en parallèle - bénévolat au dispensaire antituberculeux public, enseignement à l'école de médecine, etc.

42. La professionnalisation du spécialiste ne sera formalisée qu'après la Seconde Guerre mondiale en France avec l'institution de diplômes de spécialités. Avant 1945, la pratique spécialisée reste en métropole comme au Vietnam souvent jumelée avec une pratique généraliste; nombreux sont par ailleurs les étudiants vietnamiens qui ont eu l'occasion de se former à l'une à l'autre, disposant parfois de diplômes ou certificats de spécialisation en plus de leur doctorat.
} 
d'ailleurs oser promouvoir certains médecins libéraux ; dont Luyện. À l'époque de son installation rue de la Citadelle, il est déjà un contributeur assidu du magazine en santé Vệ sinh báo, d'où est tiré l'extrait de 1929 évoqué en prologue, magazine fondé et dirigé par une des plus grosses pharmacies françaises de la capitale coloniale à l'époque, la pharmacie Chassagne; la collusion est évidente, mais autant mettre de côté des a priori éthiques très contemporains. Comme lui, un certain nombre de médecins vietnamiens s'investissent à l'époque dans le journalisme, d'opinion ou spécialisé (magazines féminins, scientifiques), des tribunes dont ils usent probablement pour se faire un nom sur la scène philanthropique ou politique locale mais aussi à des fins d'éducation de la population. Le Dr Trân Văn Đôn (1887-?) est ainsi le fondateur en 1923 de la revue de vulgarisation scientifique Khoa học tạp chí, trois ans après avoir soutenu son doctorat à Paris sur l'ascaridiose chez l'Annamite. Installé à Saigon quelques années plus tard, après avoir accompli ses dix ans réglementaires dans le cadre de l'Assistance, il continuera d'écrire des articles de vulgarisation scientifique mais également de prodiguer des conseils de bonne santé dans différentes tribunes dont le journal féminin Phụ nữ tân van, publiant dans ce dernier pas moins de soixante articles. En 1934, il deviendra le premier vice-président vietnamien de la Société médicochirurgicale de l'Indochine, société professionnelle et savante regroupant la très grande majorité des médecins français et vietnamiens en exercice dans la péninsule.

Dans le Vệ sinh báo, Luyện parle pour sa part de pédiatrie, de maladies vénériennes bien sûr; il le fait en termes simples, insistant sur les bienfaits de la prévention au quotidien. Ses interventions ainsi que celles de ses confrères peuvent assurément susciter plusieurs interprétations de la part de l'historien; le phénomène est révélateur d'une nouvelle élite colonisée et professionnelle qui cherche à faire sa place, à se distinguer et à imposer certaines valeurs. Mais dans le cas précis de Luyện, optons pour une volonté franche de s'investir (ce qui ne veut pas dire qu'il ne cherche pas en parallèle à disposer d'une clientèle lucrative) dans l'amélioration de la santé du public en s'adaptant à ses besoins, ses attentes et à ses moyens aussi. En 1925, alors qu'il est en poste pour l'AMI à Phu Tho et sur le point de quitter le Vietnam pour la métropole, il fait ainsi paraître un vade-mecum de puériculture intitulé Sản dục chỉ nam dont il fera reproduire de larges extraits dans le Vệ sinh báo - le mémento connaîtra plusieurs rééditions successives. Un second suivra, en 1932, cette fois consacré au péril vénérien et aux moyens de le prévenir et de le 
guérir (Phong tình y án) ${ }^{43}$. En juillet 1934, il se lance même dans l'édition de son propre magazine de santé, le Bao an y báo (Revue de vulgarisation médicale).

Tirant à 1500 exemplaires (ce qui n’est pas négligeable à l'époque) jusqu'à janvier 1938, chaque numéro du Bao an y báo, de trente à quarante pages, commence toujours par un éditorial de Luyện, touchant une question d'actualité sanitaire. Il s’y exprimera sur deux sujets en priorité: la valeur et la place que devrait avoir la médecine traditionnelle dans le paysage des soins vietnamiens; et la primauté de la gratuité des soins et d'un accès le plus démocratique possible à des médicaments essentiels (c'est-à-dire traitant des problèmes répandus et ayant fait la preuve de leur efficacité). Suivent généralement quelques articles touchant la santé maternelle et infantile bien sûr de même que des conseils de bonne santé au quotidien et un formulaire thérapeutique qui propose des recettes de remèdes simples, parfois à base de produits disponibles localement. À cela s'ajoute un espace (entre quatre et huit pages) dédié aux lecteurs du journal qui peuvent, par son biais, demander conseil à la rédaction. Un système de consultation virtuelle avant l'heure en somme qui n'est pas sans rappeler les consultations épistolaires, fréquentes en Europe jusqu'à la fin du xviI ${ }^{\mathrm{e}}$ siècle au moins alors que l'accès à un médecin dûment formé reste limité ${ }^{44}$. Mentionnons enfin que près de la moitié de chaque numéro est constituée de publicités pour des cabinets médicaux, dont le sien bien sûr, des pharmacies et des médicaments, occidentaux et disponibles sans ordonnance, destinés à traiter une grande variété de symptômes et petits problèmes de santé courants.

On repère au travers de ce magazine plusieurs adaptations du modèle biomédical tel que véhiculé par le monde universitaire français et par l'AMI: une certaine insistance est ainsi mise sur le médicament comme outil de soins courant et sur l'automédication. Or les médecins coloniaux de l'époque restent très sceptiques à l'endroit du produit pharmaceutique

43. "Accusé de réception », L'Annam nouveau, n 144, 16 juin 1932. Ce manuel, découpé en trois parties qui reviennent chacune sur l'étiologie, les modes de transmission, la symptomatologie, les complications, les moyens de prévenir et guérir les principales maladies vénériennes fera l'objet d'un battage médiatique courant 1932. Les journaux insisteront tous sur le talent de vulgarisateur de son auteur et sur la valeur pragmatique, voire l'indispensabilité du texte dans tous les foyers. II était alors proposé à la vente à la pharmacie Chassagne pour la modique somme de 0,60 \$.

44. En dépouillant cette rubrique on ne peut d'ailleurs manquer de relever que certains patients virtuels habitent loin, voire très loin de la capitale coloniale (au Cambodge, au Laos parfois). Sur les consultations épistolaires voir : Joël Coste, «Les relations entre médecins et malades dans les consultations médicales françaises (milieu XVI ${ }^{e}$ siècle - début XIXe siècle) », in Les relations médecin-malade. Des temps modernes à l'époque contemporaine, Elisabeth Belmas et Serenella Nonnis-Vigilante (dir.) (Villeneuve d'Ascq: Presses universitaires du Septentrion, 2013), p. 23-48. 
(même dans le cadre d'une relation thérapeutique strictement codifiée) et promeuvent la prévention collective avant tout. Par contraste, la médecine vietnamienne, du Nord (thuốc bắc), d’obédience chinoise marquée, et surtout du Sud (thuốc nam), fondée sur une pharmacopée locale très large puisant dans une biodiversité végétale extraordinaire, a toujours misé sur le remède ${ }^{45}$. Des déviations qui peuvent être vues comme autant de marques d'indigénisation, de naturalisation de la médicalisation coloniale susceptibles de répondre aux attentes, y compris consuméristes (le Bao an y báo et bien d'autres journaux vietnamiens des années 1930 sont saturés de publicités pour des produits thérapeutiques miracle vendus par une industrie pharmaceutique occidentale mais aussi asiatique en pleine expansion ${ }^{46}$ ), mais aussi aux besoins en santé de la population colonisée et à certaines de ses mœurs en matière de santé. L'investissement journalistique de Luyện montrerait donc à la fois l'évolution des repères de santé d'une partie de la population vietnamienne et sa propre participation à cette évolution en qualité d'expert en santé, formé à l'occidentale mais travaillant dans le privé. Dans ce cadre, il dispose en effet d'une certaine marge de manœuvre; il peut critiquer subtilement le système de santé public qu'il connaît bien, revendiquer aussi, avec pragmatisme d'abord, un droit à la santé et d'abord à des soins essentiels, ce que plusieurs journaux d'opinion vietnamiens à l'époque commencent également à mettre en avant rappelant avec amertume ou ironie la faiblesse des effectifs médicaux hors des centres urbains - et l'impossibilité par conséquent, pour le gros de la population vietnamienne, d'accéder à des médicaments efficaces car très majoritairement soumis à prescription. Un droit à la santé qui pourrait passer par un recours (éclairé!) à la médecine traditionnelle (faisant ici écho à une autre tradition confucéenne forte, d'automédication éclairée), un système médical dont la modernisation, la scientifisation assurément possible et d'ailleurs en marche à l'époque, permettra à terme de l'incorporer dans un système de soins national adapté.

Une médecine traditionnelle moderne que Luyện serait même un des premiers, en 1929, à appeler notre médecine (thuốc ta $)^{47}$ promouvant avant l'heure un système de santé intégratif et défendant sur le terrain

45. Laurence Monnais, C-Michele Thompson et Ayo Wahlberg (éd.), Southern Medicine for Southern People.

46. Laurence Monnais, Médicaments coloniaux : l'expérience vietnamienne, 1905-1940 (Paris : Les Indes savantes, 2014), p. 186-90.

47. Sur la réinvention de la médecine vietnamienne de la fin de la période coloniale à nos jours et la construction de son identité voir: Laurence Monnais, C-Michele Thompson et Ayo Wahlberg (éd.), Southern Medicine for Southern People. 
des pratiques de pluralisme médical ${ }^{48}$. Henriette Bùi Quang Chiêu (19062012), première femme vietnamienne docteur en médecine (1934), allait elle aussi suivre cette voie, dans son cas en intégrant l'acupuncture dans son offre de soins à son cabinet de Saigon à la fin des années $1930^{49}$. Diplômé de Hanoi au début des années 1930, le Dr Lê Văn Phấn envoyait pour sa part une lettre au résident supérieur du Tonkin en novembre 1935 lui demandant une subvention pour l'ouverture d'une officine de propagande pour la médecine française (Việt Nam y quán), boulevard Gia Long, à Hanoi. Nous voudrions ouvrir cette " maison médicale, lui écrit-il, pour contrer l'ignorance de la masse et soustraire la population annamite à l'influence néfaste des médicastres et des charlatans [...]. Nous cherchons à faire triompher la médecine occidentale en la complétant par l'étude rationnelle de la médecine sino-annamite, c'est-à-dire par la recherche et l'étude des produits médicamenteux utilisés dans ce pays depuis des millénaires ". Renseignements pris auprès de la direction locale de la santé, le résident supérieur comprend en fait que Phấn collaborait avec une firme pharmaceutique sino-vietnamienne installée sur la route de Hué. Il lui opposa alors une fin de non-recevoir. Ce qui n’empêcherait pas ledit médecin de faire connaître son cabinet privé, en précisant qu'il avait dans cette démarche le soutien des hautes autorités et de l'élite intellectuelle du pays; il se proposait même d'offrir des consultations au tarif le plus bas à ceux et celles qui voudraient recourir à une thérapie mixte $\mathrm{e}^{50}$. Il existait probablement aussi à l'époque des cabinets mixtes (offrant les deux types de médecine) où même une clientèle blanche se serait rendue à l'occasion ${ }^{51}$. Sans jamais que ni les uns ni les autres n'en viennent à renier leur formation à la française, la valeur de leur expertise, sa qualité scientifique - et tout en affichant certaines couleurs politiques en accord avec une vision de la bonne prise en charge sanitaire et de la médicalisation de leurs futurs concitoyens.

48. Selon la définition de l'Organisation mondiale de la santé, un système de santé intégratif est un système de santé qui propose à une population donnée un accès simultané à plusieurs traditions médicales, ce de façon équilibrée et équitable, permettant des pratiques pluralistes en santé sécuritaires et efficaces. La Chine et le Vietnam constitueraient selon l'organisme des modèles du genre, mais à partir des années 1960 seulement.

49. Entrevue avec Henriette Bui, Paris, janvier 1997.

50. ANVN, centre $n^{\circ} 1$, RST 47950 , «Demande de création d'un office de propagande pour la médecine française à Hanoi formulée par Le Van Phan, médecin indochinois à Hué, 1935 »; Publicités, Báo đông pháp, n 3081, 2 au 3 novembre; n 3082, 4 au 5 novembre 1935.

51. II reste encore plus difficile de savoir dans quelle mesure des médecins français auraient pris goût à ce pluralisme. Si c'est le cas ils se sont abstenus de le crier sur les toits... 
Le Dr Nguyễn Văn Luyện aurait été un nationaliste convaincu et respecté mais ouvertement anticommuniste ${ }^{52}$. Durant le second conflit mondial, il publiera un quotidien, d'information cette fois, intitulé Tin móí. Journal quotidien d'information. Orienté sur l'actualité internationale du moment tout en continuant d'offrir des conseils d'hygiène aux femmes, pour elles et leurs enfants, le journal est saturé de publicités pour ses confrères et pour toutes sortes de médicaments. Membre fondateur du Parti démocratique du Vietnam (Đảng dân chủ Việt nam) en juin 1944, auteur de Le Vietnam une cause de la paix en 1945 et co-auteur de la constitution vietnamienne de 1946, Luyện assistera à la conférence de Dalat et accompagnera Ho Chi Minh à Fontainebleau avant de mourir le 19 décembre 1946, à Hanoi, à l'âge de quarante-huit ans, en compagnie de son fils aîné, alors qu'il cherchait à s'occuper des blessés du mouvement insurrectionnel dirigé par le Viêtminh contre les Français. Sans que l'on puisse donc préjuger de son avenir postcolonial, ni politique ni professionnel. En revanche son vade mecum de puériculture fut réédité en 1957, à Saigon cette fois, comme une référence longtemps jugée absolument incontournable pour toutes les mères vietnamiennes.

La reconstitution du parcours professionnel de Luyện, permet d'enrichir l'histoire coloniale de la santé vietnamienne mais aussi son histoire postcoloniale; elle remet en cause l'idée que la prise en charge de cette dernière a été le fait de médecins forcément passés au communisme, trempés de science pour mieux défaire le pays de l'oppression occidentale, une historiographie convenue dont l'icône reste le célèbre chirurgien Tôn Thất Tùng (1912-1982) ${ }^{53}$. Quant à la médecine que les médecins libéraux ont pratiquée après 1945 et surtout 1954, au Sud (ou à l'étranger), les paris restent ouverts mais l'écriture de cette page de l'histoire de la santé vietnamienne devra prendre en considération la possibilité qu'ils aient précocement, et de façon appuyée, participé à la réinvention de la médecine vietnamienne. En somme, ces médecins constituent des médiateurs culturels d'avant-garde, intermédiaires et acteurs d'un processus de médicalisation hybride reposant sur à la fois sur des besoins et des

52. Je tiens à remercier David Marr et plusieurs membres du Vietnamese Studies Group pour ces informations sur les dernières années de Luyện.

53. Michitake Aso et Annick Guénel, «The Itinerary of a North Vietnamese Surgeon Medical Science and Politics during the Cold War. » Science, Technology \& Society, vol. 18, n 3, 2013, p. 291-306. Selon certaines estimations d'ailleurs, seul un tiers des médecins vietnamiens formés dans la colonisation aurait rejoint le Viêt minh. 
convictions, une expertise et un pragmatisme éclairé. S'opèrent à travers eux et leurs parcours des transferts culturels complexes et à plusieurs échelles: entre la France colonisatrice et le Vietnam colonisé, entre les sphères publique et privée, entre professionnels et profanes et entre deux systèmes médicaux en pleine consolidation. 

9

\title{
L'Université indochinoise et l'œuvre culturelle de la France au Vietnam
}

\author{
Tuân Hoang Van
}

Dans l'histoire du Vietnam, l'enseignement a joué un rôle très particulier. Il y a plus de 2000 ans, sous l'influence de la domination chinoise, l'enseignement confucéen a été introduit au Vietnam. Dès le $\mathrm{xI}^{\mathrm{e}}$ siècle, la dynastie des Ly (1009-1225) a utilisé l'enseignement et les concours confucéens pour choisir ses mandarins. À partir du xve siècle, les doctrines confucéennes ont obtenu une place dominante dans l'éducation et la politique nationale du Vietnam.

Dès le début de l'occupation de la Cochinchine, les Français mettent en œuvre diverses stratégies pour supprimer l'enseignement traditionnel et installer pas-à-pas un système d'enseignement français. Sont visés la formation des interprètes et auxiliaires nécessaires à l'administration des régions occupées, ainsi que la diffusion de la civilisation française. Après l'échec d'une politique de francisation, les Français assurèrent peu à peu le développement de l'enseignement français dans un cadre préservant, au Tonkin comme en Annam, l'enseignement traditionnel, au niveau élémentaire; la formule permettait de propager l'influence française au sein de la population indigène. Puis la réforme de Beau en 1906 renforça le caractère occidental de l'enseignement, tout en préservant encore certains traits de l'enseignement traditionnel. Mais au final, la réforme promulguée par Albert Sarraut en 1917 signa la victoire de l'enseignement occidental, avec pour objectif le développement du colonialisme et de la culture française.

S’agissant de l'enseignement supérieur, les premiers pas furent la création de l'école de médecine (1902) et la fondation de l'Université indochinoise 
(1906). Mais il fallut attendre la réforme Sarraut, fixant les règles du jeu aux trois niveaux primaire, secondaire et supérieur en 1917, pour que l'enseignement supérieur puisse réellement s'organiser, avec l'instauration d'une Direction de l'enseignement et l'établissement de règlements communs.

Durant les trois décennies qui suivirent, ce système et son fleuron universitaire devaient contribuer fortement à la formation des nouvelles élites vietnamiennes et aux transferts culturels franco-vietnamiens. La formation d'agents auxiliaires indigènes, pourvus par ce système d'une bonne connaissance de la France et de sa vision des choses, fut un incontestable facteur de diffusion d'influence.

\section{La naissance et le développement de l'enseignement supérieur au Vietnam}

La tableau ci-dessous réunit les dates marquantes de l'enseignement supérieur au Vietnam, de 1902 à 1945 :

\begin{tabular}{|c|c|}
\hline Date & Evénements \\
\hline 08.01.1902 & Création de l'école de médecine \\
\hline 16.05.1906 & $\begin{array}{l}\text { Fondation de l'Université indochinoise } \\
\text { (fermeture en } 1908 \text { et réouverture en 1917) }\end{array}$ \\
\hline 08.07.1917 & Création de la direction de l'enseignement supérieur de l'Indochine \\
\hline 15.09.1917 & Création de l'école vétérinaire \\
\hline 15.10 .1917 & Création de l'école supérieure de pédagogie \\
\hline 15.10 .1917 & $\begin{array}{l}\text { Création de l'école de droit et d'administration } \\
-18.09 .1924 \text {, école des hautes études indochinoises, } \\
-11.09 .1931 \text {, école supérieure de droit }\end{array}$ \\
\hline 21.12.1917 & $\begin{array}{l}\text { Déclaration du règlement général de l'Instruction publique } \\
\text { (Code de l'Instruction publique) }\end{array}$ \\
\hline 17.03.1918 & Création de l'école pratique d'agriculture \\
\hline 21.03.1918 & Création de l'école supérieure d'agriculture et de sylviculture \\
\hline 25.12.1918 & $\begin{array}{l}\text { Déclaration du règlement général de l'enseignement supérieur de } \\
\text { I'Indochine }\end{array}$ \\
\hline 02.11 .1920 & Création de l'école de commerce \\
\hline 27.10 .1924 & Création de l'école des beaux-arts \\
\hline 28.07.1941 & Création de l'école supérieure des sciences \\
\hline
\end{tabular}

Le développement de l'enseignement supérieur au Vietnam s'est déroulé en trois temps. 
La première période va de 1902 à 1917: première étape de l'enseignement supérieur au Vietnam avec la naissance de l'école de médecine (1902) et de l’Université indochinoise (1906). Bien que la création de l’Université indochinoise en 1906 n’ait été qu'une mesure politique, elle fut considérée comme un point important dans l'histoire de l'enseignement supérieur en Indochine. Par son modèle, cette université a établi la base d'un système d'enseignement supérieur en Indochine pour l'avenir.

La deuxième période s'étend de 1917 à 1930: réouverture de l'université de Hanoi et organisation d'un système d'enseignement supérieur. La création d'une Direction de l'enseignement supérieur de l’Indochine (juillet 1917), la déclaration du règlement général de l'Instruction publique en Indochine (décembre 1917), la déclaration du règlement général de l'enseignement supérieur (décembre 1918) furent les étapes fondamentales de l'installation et du développement d'un système de l'enseignement supérieur en Indochine. Entre 1917 et 1925, s’ajoutant à l'école de médecine qui se développait peu à peu, fut créée toute une gamme d'établissements supérieurs: l'école vétérinaire, l'école supérieure de pédagogie, l'école de droit et d'administration (1917), l'école supérieure d'agriculture et de sylviculture, l'école pratique d'agriculture (1918), l'école de commerce (1920) et l'école des beaux-arts (1924).

La troisième période mène de 1930 à 1945. Elle consacre le développement du système. Malgré des difficultés budgétaires, l'administration coloniale maintient son intérêt pour le supérieur et le fait encore progresser dans son statut: l'école supérieure de médecine et de pharmacie et l'école supérieure de droit sont transformées en facultés et rattachées à l'université de Paris. En 1941, la naissance de l'école supérieure des sciences parachève le développement de l'enseignement supérieur en Indochine. L’Université indochinoise posséde désormais une structure identique à celle des autres universités de la métropole.

Pour bien apprécier le processus qui vient d'être rappelé, il convient de faire référence à ce que fut de manière générale la politique de la France en matière d'enseignement, supérieur notamment, concernant ses colonies. La situation de l'Indochine sur ce plan illustre l'importance qu'on lui reconnaît, autant qu'aux deux autres fleurons de l'Empire que sont l'Algérie et le Sénégal. Très riche en ressources naturelles, doté d'un grand potentiel, la colonie indochinoise représente aussi une voie d'accès à la Chine.

En 1906, lors de la fondation de l'Université indochinoise, il n'existait dans l'Empire qu'une faculté, en Algérie, la plus ancienne colonie de la France. Dans les autres colonies, l'enseignement était très limité. Dans les 
régions d'Afrique occidentale par exemple, il n'y avait qu'une seule école normale, à Ponty (créée en 1903).

En Algérie, des écoles supérieures avaient été créées depuis le milieu du XIX ${ }^{\mathrm{e}}$ siècle $^{1}$ : d'abord, l'école de médecine (par le décret du 4 août 1857), ensuite les écoles de droit et de sciences. Puis, la loi relative à l'enseignement supérieur en Algérie fut votée au Sénat, le 20 décembre 1879. Selon son article premier : "Il est créé, à Alger, à côté de l'école de médecine et de pharmacie, déjà existante, une école préparatoire à l'enseignement du droit, une école préparatoire à l'enseignement des sciences et une école préparatoire à l'enseignement supérieur de lettres ${ }^{2}$. " Jusqu'au début du xx $x^{\mathrm{e}}$ siècle, le système d'écoles supérieures en Algérie fut organisé sur la base très solide de l'enseignement secondaire: «Dans toute Algérie, l'enseignement secondaire était déjà fortement organisé; avec deux lycées (Alger et Constantine), dix collèges communaux et quatre institutions libres, il totalisait, en 1878, 3142 élèves $^{3}$. " L’enseignement primaire et secondaire en Algérie avait été organisé selon le modèle de la France après les réformes de Jules Ferry depuis les années 1880. Et « les écoles supérieures d’Alger ont compté [...] au 15 janvier 1909 près de 1605 étudiants ${ }^{4}$ ». En 1909, l'administration française décida de créer l'université d'Alger sur la base de l'ensemble des écoles supérieures qui existaient déjà. « Le projet de loi (déposé le 11 mai 1909) constituant en Université les écoles d'enseignement supérieur en Algérie est voté par la chambre des députés le 5 juillet 1909 et devient, après modification, la loi du 30 décembre 1909 qui transforme les écoles supérieures en facultés et les réunit en université: "Art.1 - Les écoles supérieures d’Alger reçoivent le titre de facultés et sont constituées en université [... $]^{5}$ »

Le développement de l'enseignement dans les colonies est une politique qui fut attentivement soupesée par la France, et s'adapta à la situation de chaque colonie. Ce qui réussit en Algérie où l'enseignement accueillait un grand nombre d'élèves français ne pouvait se dupliquer en Indochine où la population métropolitaine était faible. D’après le chiffre donné par Blanchard de la Brosse : «Jusqu'aux années 1920, il y a environ 500000 Français en Algérie contre 16000 Français en Indochine ${ }^{6}$ ». En

1. République Française, université d'Alger 1909-1959, p. 21.

2. Jean Mélia, Histoire de l'université d'Alger, La maison des Livres, Alger, 1950, p. 56.

3. République Française, université d'Alger 1909-1959, p. 24.

4. Jean Mélia, Histoire de l'université d'Alger, op. cit, p. 57.

5. République Française, université d'Alger 1909-1959, p. 59.

6. P. de la Brosse, Une année de réformes dans l'enseignement public en Indochine (1924-1925), Hanoi, direction de l'Instruction publique, 1925, p. 19. 
revanche, en Indochine, le développement de l'enseignement, et celui de l'enseignement supérieur en particulier, fut impulsé par des intérêts de la France et par les exigences du peuple du protectorat, en particulier dans la période suivant la Première Guerre mondiale.

Après cette dernière, la France fait face à de multiples difficultés ${ }^{7}$ : 1,4 million personnes tuées et des centaines de milliers blessées. Parallèlement, la production s'est effondrée. Ces difficultés imposaient des dispositions urgentes pour le rétablissement du pays. Une des plus importantes mesures fut le renforcement de l'exploitation des colonies. On attendait que l'Indochine continue de jouer le rôle de source privilégiée des apports financiers ${ }^{8}$. Ainsi, selon l'auteur de Indochine années vingt: Le rendez-vous manqué (1918-1928), durant cette période, on souhaitait que l'Indochine devienne un rempart de la France au lieu d'un balcon sur l'océan Pacifique, qu'elle soit le relais, le moteur économique de la France après la guerre. " Représenter la France dans le Pacifique, être son relais et devenir son moteur économique en Extrême-Orient ne peut se faire en opprimant sans espoir un peuple de 20 millions d'âmes avec seulement 20000 ressortissants ", écrit Patrice Morlat ${ }^{9}$.

Par ailleurs, après la guerre, la France fait face aux pressions qui s'exercent à travers le monde concernant sa responsabilité vis-à-vis de colonies qui avaient contribué à sa victoire. En conséquence, au lendemain de la guerre, selon Antoine Léon, deux nouvelles exigences apparaissent dans un projet de loi fixant, le 28 octobre 1919, l'organisation de l'instruction publique dans les colonies. Tout d'abord, " un devoir de justice envers les fidèles populations coloniales qui n’ont épargné ni leur sang, ni leur argent... ». En second lieu, " un devoir de dignité " imposé par la conjoncture internationale, le monde entier étant attentif " aux principes qui doivent inspirer la conduite des puissances coloniales envers les populations dont elles ont la charge matérielle et morale ${ }^{10}$ ". Patrice Morlat le dit franchement: "La France était redevable à ses colonies de l'avoir aidée à gagner la guerre contre l'Allemagne ${ }^{11}$. "

7. Voir: Georges Duby, Histoire de la France de 1852 à nos jours, Editions Larousse, Paris, 1999, p. 231. et Philippe Bernard, La fin d'un monde 1914-1929, Paris, Le Seuil, 1975, p. 110-111.

8. L'Indochine a contribué toute seule pour environ la moitié du montant des finances qui furent fournies par toutes les colonies de la France (13.8 millions francs sur un total de 27.4 millions pendant la Grande Guerre 1914-1918). Voir aussi Albert Sarraut, La mise en valeur des colonies françaises, Payot, Paris, 1923.

9. Patrice Morlat, op. cit, p. 18.

10. Antoine Léon, Colonisation enseignement et éducation, Editions L'Harmattan, Paris, 1991, p. 28.

11. Patrice Morlat, op. cit, p. 17. 
Pour faciliter l'exploitation et développer ses colonies, il faut obtenir une coopération étroite avec les populations indigènes. C'était la base même sur laquelle on mit en place la politique d'association franco-indigène à cette époque. En Indochine, cette politique fut instaurée et appliquée par un grand politique, Albert Sarraut qui y effectua son deuxième mandat de gouverneur général. En raison de toutes les contributions de l'Indochine pendant la guerre, des attentes de la France concernant cette colonie et dans le contexte commun de la politique de la coopération franco-indigène, l'enseignement était un élément crucial. Pendant les années 1920 et 1930, il y eut des réformes ou des modifications de la part des gouvernements, mais les principes de l'enseignement d'Albert Sarraut furent toujours considérés comme la ligne directrice pour l'organisation et le fonctionnement de l'enseignement général et de l'enseignement supérieur en Indochine.

Le service de l'enseignement et de la jeunesse, dans le rapport du commissaire fédéral à l'enseignement, confirme que: "Dès 1917, en pleine guerre mondiale, la France a constitué en Indochine les premiers éléments de l'université de Hanoi. Mais c'est avec la création successive des facultés ou écoles supérieures de médecine et de pharmacie réorganisée en 1935, de droit, organisée en 1931, des sciences fondée en 1941 que l'université de Hanoi a été organisée sur le modèle de nos universités françaises, pourvue du personnel magistral qualifié par ses titres et délivrant les diplômes d'État comme dans la Métropole ${ }^{12}$. "

Nombre d'étudiants de l'université de $\operatorname{Hanoi}^{13}$ :

\begin{tabular}{lccc} 
Année scolaire & Nombre d'étudiants & Année scolaire & Nombre d'étudiants \\
\hline $1918-1919$ & 438 & $1938-1939$ & 457 \\
$1919-1920$ & 448 & $1939-1940$ & 573 \\
\hline $1920-1921$ & 516 & $1940-1941$ & 602 \\
\hline $1921-1922$ & 525 & $1941-1942$ & 834 \\
$1929-1930$ & 541 & $1942-1943$ & 1050 \\
\hline $1930-1931$ & 352 & $1943-1944$ & 1575
\end{tabular}

12. Centre des Archives nationales d'Outremer, Nouveaux fonds, carton 147, dossier 1323 : Rapport du Commissaire fédéral à l'Enseignement.

13. Les chiffres sont tirés de : Đại học Quốc gia Hà nội, Một thế kỉ phát triển và trưởng thành (" Un siècle de l'Université nationale de Hanoi »), éd. Université nationale de Hanoi, Hanoi, 2006; gouvernement général de l'Indochine, Rapports au conseil de gouvernement de l'Indochine; gouvernement général de l'Indochine, Annuaire administratif de l'Indochine; gouvernement général de l'Indochine, Annuaire statistique de l'Indochine, premier volume 1913 à 1922, Imprimerie d'Extrême-Orient, Hanoi, 1927 ; Phan Trọng Báu, Giáo dục Việt Nam thời cận đại («L'éducation au Vietnam dans l'époque moderne »), éd.de l'Éducation, Hanoi, 2005. 


\section{Les objectifs de l'enseignement supérieur au Vietnam}

L’enseignement général et l'enseignement supérieur au Vietnam, avaient pour buts principaux:

- de répondre aux demandes des Vietnamiens concernant l'enseignement ;

- de former des agents pour les services généraux en colonie ;

- de propager l'influence culturelle et scientifique française.

Avant l'époque coloniale, le Vietnam était profondément influencé par la culture chinoise: par sa langue, sa pensée et son enseignement confucéen. Pour la France, cela constituait un gros obstacle à la diffusion de son influence culturelle. En effet, la suppression de l'influence chinoise et la francisation de la culture vietnamienne, étaient une lourde tâche, difficile à réaliser. Dès le début, les Français l'avaient bien compris et, parmi leurs stratégies, l'enseignement devint un moyen de faire "la conquête de l'esprit du peuple vietnamien ».

Dans l'histoire du Vietnam, l'enseignement a toujours joué un rôle particulier. Pendant des centaines d'années, l'enseignement et les concours confucéens étaient la voie la plus importante (sinon la seule et unique) pour obtenir un poste dans l'administration. C'était en effet le principal instrument des dynasties vietnamiennes pour former, nommer leurs fonctionnaires et assurer la fidélité des élites. L'enseignement était donc un moyen très efficace pour la conquête morale du peuple.

Pour l'administration française, la propagation de la culture française représentait une tâche importante, permanente de l'enseignement, et de l'enseignement supérieur en particulier. Antoine Léon, dans son ouvrage Colonisation enseignement et éducation a cité dix objectifs que les Français souhaitaient atteindre à travers l'instruction publique, dont plusieurs objectifs concernant la mission de propagation de la culture française ${ }^{14}$.

$\mathrm{Au}$ Vietnam et en Indochine en général, cette mission fut exprimée dans l'opinion de toutes les hautes autorités françaises. Selon Paul Doumer, le développement de l'enseignement serait « une des formes les plus efficaces et les plus honorables de notre pénétration ${ }^{15}$ ».

Henri Gourdon, directeur de l'Instruction publique de l'Indochine, l'exprime ainsi: "L'Université indochinoise s'efforcera de créer en

14. Voir Antoine Léon, Colonisation, enseignement et éducation, L'Harmattan, Paris, 1991, p. 19-35.

15. CAOM, GGI. 6779 : École de médecine - création et organisation 1898-1913. 
Indochine un véritable centre de culture européenne, et contribuera ainsi à hâter l'évolution intellectuelle de nos protégés et à accroître l'influence de notre pays en Extrême-Orient ${ }^{16}$. "

C'était aussi la grande mission de l'Université indochinoise: "Cette Université est destinée à répandre en Extrême-Orient, surtout par l'intermédiaire de la langue française, la connaissance des sciences et des méthodes européennes ", dit le premier article de l'arrêté créant l'Université en $1906{ }^{17}$.

En 1917, sous la direction d'Albert Sarraut, une grande réforme de l'enseignement fut mise en application au Vietnam. L’enseignement supérieur était au sommet d'un système d'enseignement à trois degrés: primaire, secondaire et supérieur. Une des raisons de cette réforme, était l'influence exercée par l'Allemagne au Vietnam, à travers des ouvrages publiés en chinois et traduits en vietnamien. Pour Albert Sarraut, l'enseignement supérieur et l'université indochinoise devaient non seulement former des cadres auxiliaires indigènes, mais un symbole de l'influence de la civilisation française: "L'œuvre civilisatrice " de la France. Selon Trinh Van Thao, Albert Sarraut ambitionne d'atteindre, par l'enseignement, le double objectif fixé par Jules Ferry: doter la colonie de cadres à la hauteur de son développement économique et culturel, et aussi, faire de l'Indochine une sorte de vitrine de l'œuvre civilisatrice de la France en Asie ${ }^{18}$.

Pendant la Seconde Guerre mondiale, l'université de Hanoi devenait un champ culturel disputé par les Français, les Japonais et les Vietnamiens. En raison de la guerre, de nombreux élèves français furent admis à l'université de Hanoi. Cela constitua un facteur d'échange et de transfert culturel entre les Français et les Vietnamiens.

\section{L'accueil par les Vietnamiens de ce nouveau système d'enseignement}

$\mathrm{Au}$ Vietnam, dès le milieu du xix ${ }^{\mathrm{e}}$ siècle, il existait un désir de réformer le pays, notamment par la transformation de l'enseignement et la modification des concours confucéens. Mais, en raison de la crise sociale provoquée par la conquête des Français, ces exigences n'ont pas pu être mises en œuvre.

16. CAOM - GGI. 7706 : Arrêtés et décrets concernant l'Instruction publique depuis la création de la direction générale.

17. CAOM - GGI. 48042: Création et organisation de I'Université Indochinoise.

18. Trinh Van Thao, L'école française en Indochine, Karthala, Paris, 1995, p. 77. 
Malgré quelques oppositions, le nouveau système d'enseignement organisé par les Français au Vietnam, a donc obtenu le soutien des Vietnamiens. Il faut préciser que l'enseignement établi par les Français avait un caractère pratique. Il concentrait son attention sur les capacités pratiques des élèves dans des domaines concrets. L'enseignement théorique serait limité. Autrement dit, ces écoles supérieures fonctionneraient plutôt comme des écoles professionnelles et non comme les universités métropolitaines. Leur but était de former des cadres, des agents auxiliaires, des techniciens, des ouvriers qualifiés qui serviraient à l'exploitation coloniale. Ceci a été confirmé par les hautes autorités françaises. Maurice Cognacq, directeur de l'Instruction publique de l'Indochine, exprime clairement son opinion et celle de l'Administration elle-même:

Nous n'avons voulu faire ni des savants de laboratoires, ni des fabricants d'utopies. - l'Université indochinoise conçue sur un type semblable à ses sœurs voisines d'Extrême-Orient, mais nourrie du lait plus substantiel de la science française, mère robuste et souriante du progrès humain, l'Université indochinoise s'essaiera à remplir la tâche féconde d'organiser pour la lutte mondiale une Indochine prospère et éclairée. Il suffit pour l'heure présente de donner aux jeunes gens les connaissances spéciales et l'habileté qui feront d'eux d'excellents instruments de travail; plus tard nos successeurs pourront orienter vers d'autres besoins plus relevés les aspirations plus désintéressées d'une élite déjà transformée; c'est alors que, suivant la définition du président Wilson de la grande République alliée, l’Université fera des jeunes gens qu'elle accueillera " plus que des hommes de métier et d'habiles professionnels » - l'heure n'était pas encore venue de développer chez un peuple qui doit conquérir, de haute vigueur, sa place sur l'immense marché du monde, des tendances trop accusées à l'esprit spéculatif qui si souvent brisent les ressorts de l'action ${ }^{19}$.

Un tel enseignement supérieur ne pouvait pas complètement satisfaire les lettrés, les intellectuels locaux qui étaient formés par l'enseignement confucéen, système consacré à l'enseignement de la morale et de la philosophie. Malgré tout, une partie des lettrés se montrèrent favorables à la création de ce nouvel enseignement supérieur.

À partir de 1919, date de suppression de l'enseignement confucéen (le dernier concours mandarinal a été organisé à Hué en 1919), les Vietnamiens n'avaient pas d'autre choix que l'enseignement français.

19. «Về Việc ngự giá bắc tuần », Nam Phong tạp chí, n 10 ; discours de M. le Dr Cognacq directeur de l'enseignement supérieur, inspecteur général de l'Instruction publique - à l'occasion de l'inauguration de l'Université indochinoise à Hanoi, le 28 avril 1919. 
Les jeunes Vietnamiens suivirent ce nouvel enseignement avec toute leur ferveur, toute leur passion, et ils l'adoptèrent très vite. En outre, les intellectuels vietnamiens, dont les anciens grands lettrés, s'intéressaient à ce nouvel enseignement et même le soutenaient. Cela s'exprimait à travers des dizaines d'articles publiés dans les revues ou les journaux locaux, Nam Phong tap chi (1917-1934) en particulier. Bien qu'ils ne soient pas toujours d'accord avec l'administration sur le modèle et la méthode de l'université, des auteurs, comme Phạm Quỳnh ${ }^{20}$, par exemple, ont toujours conseillé aux jeunes Vietnamiens de tirer profit de leurs études et d’appliquer tout ce qui était enseigné par l’université.

Dans son article intitulé Trường Đại học ("L’Université ») publié dans la revue Nam Phong en septembre 1917, Phạm Quỳnh souhaite une véritable université au Vietnam, comme celle de Paris. Il imagina ainsi l'université de Hanoi:

Un grand et superbe édifice se situe dans un angle de la rue où, chaque jour, les professeurs avec quantité de livres entre les mains, [...] et les étudiants âgés d'une vingtaine d'années, main dans la main, discutaient [...]. Dans ce bâtiment scientifique, les enseignements se dérouleraient partout dans les salles de cours, l'enseignement de la philosophie dans une salle et celui de la littérature dans une autre. Les étudiants seraient captivés par les paroles des professeurs $[\ldots]^{21}$

Cependant, il était d'accord sur le fait qu'à ce moment-là, l'enseignement pratique était plus important que l'enseignement théorique. Il conseilla donc d'être:

[...] déterminé à choisir l'enseignement pratique pour apprendre rapidement des techniques nécessaires pour vivre dans le monde moderne [...]. Quant à nos jeunes gens, la plus importante et urgente mission était de leur enseigner des professions utiles au développement du pays ${ }^{22}$ ».

20. Phạm Quỳnh (1892-1945), est un écrivain, journaliste et homme politique vietnamien. II est né à Hué en 1892. En 1908, il est un des premiers élèves vietnamiens à avoir obtenu le diplôme d'études primaires supérieures; il fut nommé à un poste auxiliaire à l'École française d'Extrême-Orient. En 1917, il est cofondateur de la revue Nam Phong (Vent du Sud). En 1932, il est nommé Ngự tiền văn phòng (secrétaire général), puis Thượng thư Bộ học (ministre de l'Éducation nationale) et Thượng thư Bộ lại (ministre de l'Intérieur) de la Cour de Hué. En 1922, pour sa visite de Paris à l'occasion de l'exposition internationale à Marseille, il fait quatre discours, dont « Un problème d'éducation des races. Comment doit être faite l'éducation des Annamites par la France? », à l'Académie des sciences morales et politiques, dans sa séance du 22 juillet 1922 (voir : Phạm Quỳnh, Nouveaux Essais franco-annamites, Huế, éd. Bui-Huy-Tin, MCMXXXVIII, p. 71-86).

21. Phạm Quỳnh, « Trường Đại học » (L'Université), Nam Phong tạp chí, n 3 (9.1917), p. 145-152.

22. Phạm Quỳnh, « Trường Đại học » (« L'Université »), Nam Phong tạp chí, số 3 (9.1917), p. 145-152. 
Selon lui, le nouvel enseignement supérieur saura bien satisfaire à l'exigence immédiate du développement du Vietnam: il s'agit-là d'une étape nécessaire vers la construction et l'accomplissement de l'enseignement supérieur dans l'avenir.

Lors d'une autre occasion, Phạm Quỳnh exprima son opinion sur l'enseignement supérieur français au Vietnam lorsqu'il exhorta les étudiants vietnamiens en exprimant son espoir:

Les Français, disaient-ils, se décident enfin à fonder une école supérieure; ils se décident enfin à donner aux fils d'Annam un enseignement moins terre à terre que celui qui a été donné jusqu'ici à leurs subalternes des bureaux. Certes, ils ne vous enseigneront pas tout, ils conserveront toujours les secrets qui font leur puissance - car nos lettrés croyaient fermement que les Français, comme les génies des légendes antiques, possédaient des secrets qui font leur puissance, secrets qu'ils ne divulgueraient jamais aux autres peuples - mais le peu qu'ils vous enseigneront, il faut s'empresser d'en profiter; il faut en profiter, mes jeunes amis, pour le bien du pays ${ }^{23}$.

\section{L'influence de l'enseignement supérieur sur les Vietnamiens}

L'enseignement supérieur se développait de plus en plus, et il contribua considérablement au progrès de la société vietnamienne, notamment par des transferts culturels entre Occident et Orient, entre modernité et tradition, au Vietnam.

Premièrement, l'enseignement supérieur occidental a fait changer l'opinion des Vietnamiens sur l'enseignement. L'enseignement confucéen n'avait qu'un seul but: devenir mandarin. Au début, la plupart des étudiants vietnamiens choisissaient deux domaines dans leurs études: médecine ou droit. Le changement s'exprimait par l'orientation d'un grand nombre d'étudiants vers d'autres domaines: études vétérinaires, agriculture, sylviculture, commerce, travaux publics, autant de domaines très importants, nécessaires au développement du pays. Ce changement représentait une grande réforme de l'enseignement au Vietnam. Ainsi, on peut affirmer que l'enseignement occidental conduisit à la naissance et au développement des services professionnels au Vietnam.

Deuxièmement, le nouvel enseignement amenait des changements de méthodes et de matières pédagogiques. Les bibliothèques, les laboratoires

23. Phạm Quỳnh, Nouveaux Essais franco-annamites, éd. Bui Huy Tin, Hué, MCMXXXVIII, p. $28-29$. 
et les cités universitaires, témoignent des changements importants survenus dans l'histoire de l'enseignement au Vietnam. Par exemple, la bibliothèque de l'école de médecine et de pharmacie " contient 5000 volumes, et est abonnée aux 70 principaux périodiques médicaux et scientifiques du monde entier. L'école de médecine reçoit également un exemplaire de toutes les thèses soutenues dans toutes les facultés de la métropole et de l'Algérie ${ }^{24}$ ". La bibliothèque universitaire, selon le rapport de la direction de l'Instruction publique de l'Indochine, en 1936, comprend 10756 ouvrages repartis en trois sections: section de droit (1889 ouvrages), section de médecine et pharmacie (4067 ouvrages) et section de commerce et d'agriculture (10756 ouvrages) ${ }^{25}$.

Troisièmement, l'image des professeurs français à l'université de Hanoi était prestigieuse, avec les beaux symboles gravés dans le cœur et l'esprit des Vietnamiens. L'encadrement enseignant fut choisi rigoureusement et plusieurs professeurs furent de grands savants ou de grands artistes, comme Alexandre Yersin, Le Roy des Barres, Armand Degorce, Maurice Cognacq, Pierre Huard, Jacques Meyer May, Henri Gaillard, Victor Tardieu, etc. Plusieurs anciens étudiants de l'Université indochinoise, dans leurs mémoires, ont exprimé leur sympathie, leur respect pour les talents et les qualités morales des professeurs français ${ }^{26}$.

On peut remarquer que, pendant les années de 1934 à 1940, l’Université de Paris a envoyé une dizaine de professeurs délégués au Vietnam pour présider des examens de fin d'études ou des jurys de thèse à l'Université indochinoise (Gilbert Gidel, René Maunier, Gaétan Pirou, Jean Escarra, René Cassin de la faculté de droit; Émile Brumpt, Champy, Fernand Lemaître, Louis Pasteur Vallery-Radot... de la faculté de médecine). C'était une bonne manière de favoriser l'influence culturelle et scientifique française. Parallèlement à leur principale mission, ils donnèrent des cours aux étudiants indochinois, organisèrent des séminaires scientifiques ou des conférences en se rendant dans les pays voisins, au Siam, en Chine, au

24. Archives nationales, dossier AJ 16. 6684: enseignement médical à l'étranger rattaché à la faculté de médecine de Paris 1929-1957/Histoire de l'école de médecine et de pharmacie de l'Indochine.

25. Centre d'archives nationales d'outre-mer, fonds Gouverneur général de l'Indochine, dossier 47991: Rapport sur le fonctionnement de la direction de l'Instruction publique année scolaire 1935-1936.

26. Mémoire du Dr Nguyen Luu Vien, «Quelques souvenirs d'un étudiant de l'école de médecine », Revue de médecins, Canada, $n^{\circ} 110$ (2.1991). Dans son mémoire, Nguyen Luu Vien raconte plusieurs souvenirs concernant les professeurs français à l'école de médecine : le directeur de l'école et professeur de parasitologie Henri Gaillard et le professeur de chirurgie abdominale Meyer-May, Parmi les Parisiens; le professeur Charles Massias et le professeur André Blondel, ancien interne lauréat des hôpitaux de Paris, un artiste romantique qui se retrouve dans son désordre, etc. 
Japon, aux Philippines. Par exemple, en 1935, après sa mission à Hanoi, le professeur Émile Brumpt a rendu visite à plusieurs institutions éducatives et scientifiques en Chine et au Japon. Il a fait quatre conférences, dont l'une à l'Université franco-chinoise à Peiping, deux à l'université de l'Aurore et une à l'Alliance française à Shanghai. Les professeurs Fernand Lemaître et Louis Pasteur Vallery-Radot eux-mêmes ont fait aussi des conférences dans le Yunnan (Chine), après leur principale mission en Indochine. Au travers de leurs activités, les nouvelles réalisations scientifiques françaises furent présentées largement dans le monde asiatique, surtout en Extrême-Orient. Sans aucun doute, on peut dire que ces professeurs délégués étaient des ambassadeurs de la culture et des sciences de la France. Ils contribuaient de façon importante à la présentation, à la propagation de l'influence française, de la culture et des sciences occidentales en Extrême-Orient. Le professeur Pasteur Vallery-Radot, dans le rapport sur sa mission à Hanoi en 1939, a affirmé que :

L'école de médecine de Hanoi peut être un de nos principaux moyens d'action pour développer l'influence française dans les pays voisins ${ }^{27}$.

La présence d'un personnel enseignant de qualité a permis à l'école d'organiser des cours complémentaires et de perfectionnement dans les services de clinique et dans les laboratoires, destinés aux médecins d'Indochine et de l'étranger, et aux étudiants déjà avancés dans leur scolarité, écrit un observateur français ${ }^{28}$.

\section{L'influence de la culture française sur celle du Vietnam}

Quant à l'influence de la culture française sur celle du Vietnam, on peut citer deux domaines où elle apparaît très clairement: la littérature et les beaux-arts.

En littérature, c'est l'émergence du mouvement Thơ Mới (" Nouvelle poésie ») dans les années 1930, réunissant de grands auteurs, tels Xuân Diệu, Thế Lữ, Vũ Đình Liên, Huy Cận, formés par l'enseignement français et influencés profondément par la littérature romantique française. La

27. Archives nationales, dossier AJ 16. 6684 enseignement médical à l'étranger rattaché à la faculté de médecine de Paris 1929-1957: Rapport de M. Pasteur Vallery - mission de l'Indochine.

28. Archives nationales, dossier AJ 16. 6684 : enseignement médical à l'étranger rattaché à la faculté de médecine de Paris 1929-1957: Histoire de l'école de médecine et de pharmacie de I'Indochine. 
naissance et l'influence du Thơ Mới soulignent la victoire de la littérature romantique française sur la littérature chinoise, surtout la poésie des Tang, au Vietnam. Ce fut un grand virage, une vraie réforme dans l'histoire de la littérature vietnamienne.

Dans le domaine artistique, l'école créée en 1924 apporta une grande contribution à la naissance et au développement des beaux-arts modernes au Vietnam. Sur vingt ans (1924-1945), cette école a formé des centaines de peintres, sculpteurs, architectes, dont de grands noms de l'art vietnamien moderne comme Nguyên Phan Chánh, Georges Khánh, Tô Ngọc Vân, Lê Phổ... ${ }^{29}$ Les travaux des étudiants de l'école des beauxarts furent présentés et hautement appréciés lors d'expositions dans le pays et à l'étranger: exposition internationale des colonies à Paris en 1931, exposition de Rome en 1932, expositions encore en Italie en 1934, en Belgique en 1935, au Japon en 1940. Tô Ngoc Vân, diplômé de l'école (promotion 1926-1931), obtint la médaille d'or à l'exposition des beaux-arts de Paris en 1931, et un satisfecit d'honneur à l'exposition de l'Association des peintres français (Salon de Paris, en 1932). Tô Ngọc Vân, premier directeur vietnamien de l'école des beaux-arts, et ses collègues vietnamiens, contribueront au développement des beaux-arts vietnamiens modernes et au développement de la formation des beaux-arts au Vietnam, suite à la ligne tracée par leur maître, Victor Tardieu.

Dans les annales de l'école des beaux-arts de Hanoi (1990), les auteurs ont clairement reconnu la grande contribution de l'école au développement des beaux-arts au Vietnam en affirmant que la création d'une école des beaux-arts:

est un jalon marquant le premier échange entre l'Orient et l'Occident dans l'histoire des beaux-arts contemporains et modernes du Vietnam [...]. Si auparavant, il n'y avait pas d'école, la formation se faisant seulement par le compagnonnage dans les corporations, ou par "la transmission de père en fils”..., il existe désormais une école de formation d'artistes de niveau universitaire prompts à l'approche des sciences et des arts de l'humanité. C'est un établissement scientifique destiné à éveiller et en enrichir l'art traditionnel, un pont entre les arts traditionnels et les arts modernes, un point de rencontre entre l'art oriental et l'art occidental. Ainsi, en parlant de l'histoire des beaux-arts contemporains et modernes du Vietnam, ne peut-on pas passer sous silence le rôle de l’École supérieure des beaux-arts de Hanoi. Berceau de générations de

29. Voir: Les peintres de l'École supérieure des beaux-arts de l'Indochine, éd. Beaux-Arts, $2^{\mathrm{e}}$ éd, Hanoi ; Lê Quôc Bảo (Dir.), Trường Đại học Mỹ thuật Hà Nội : 1925-1990 ("L'école des beaux-arts de Hanoi : 1925-1990»), éd. Beaux-Arts, Hanoi, 1990. 
peintres, sculpteurs, d'auteurs et d'œuvres qui ont fait l'histoire des beaux-arts modernes du Vietnam ${ }^{30}$.

En outre, l'Université indochinoise attira des centaines d'étudiants étrangers, surtout des jeunes Chinois. La présence des élèves étrangers exprimait le rôle de l'université non seulement en Indochine, mais aussi en Extrême-Orient, selon le souhait du professeur Émile Brumpt dans son rapport de 1936, après sa mission à l'école de médecine de Hanoi, disant: "Je suis convaincu que l'école d'Hanoi pourrait devenir un centre de haute culture en Extrême-Orient ${ }^{31}$. " Son souhait était cohérent avec celui de Paul Doumer qui espérait que les étudiants étrangers " seraient plus tard de précieux agents de la France dans les pays voisins. Leur admission présenterait un sérieux intérêt pour la propagation de l'influence et des méthodes scientifiques françaises $[\ldots . . .]^{32}$ »

Le tableau ci-dessous montre aussi que le nombre d'étudiants français à l'Université indochinoise a augmenté considérablement pendant la Seconde Guerre mondiale. Durant l'année scolaire 1924-1925, il y a 11 étudiants français sur un total de 415 étudiants à l'Université indochinoise (soit 2,6 \%). En 1938-1939, à la veille de la guerre, le nombre d'étudiants français était de 88 sur 732 (soit $12 \%$ ). Pendant l'année scolaire 1941-1942, le nombre de Français à l’Université indochinoise a augmenté jusqu'à atteindre les 218 (soit 19,8 \%) et, enfin, au cours de l'année scolaire 1943-1944, le chiffre était de 371/1528 (soit $24 \%$ ).

Nombre d'étudiants étrangers à l'Université indochinoise ${ }^{33}$

\begin{tabular}{lcccc} 
Années & \multicolumn{4}{c}{ Nombre d'étudiants (origine géographique) } \\
\hline & Indochinois & France & Chine & Autre \\
\hline $1924-1925$ & 396 & 11 & 8 & \\
$1938-1939$ & 633 & 88 & 11 & \\
$1941-1942$ & 858 & 218 & 20 & 5 \\
$1943-1944$ & 1146 & 371 & 11 &
\end{tabular}

30. Lê Quôc Bảo (Dir.), Trường Đại học Mỹ thuật Hà Nội: 1925-1990 (« L'école des beaux-arts de Hanoi : 1925-1990 »), Éditions Beaux-Arts, Ha Noi, 1990, p. 15-16.

31. Archives Nationales, dossier AJ 16. 6684 : Enseignement médical à l'étranger rattaché à la Faculté de médecine de Paris 1929-1957; Rapport de M. Brumpt 1936.

32. Université Indochinoise, Annales de l'École supérieure de médecine et de pharmacie de I'Indochine, t. IX, 1944, Imprimerie d'Extrême-Orient, Hanoi, 1944, p. 8.

33. Les chiffres tirés de : Gouvernement général de l'Indochine, Rapports au Conseil de gouvernement de I'Indochine; Annuaire administratif de l'Indochine; Trinh Van Thao, L'école française en Indochine, Editions Karthala, Paris, 1995. 
En 1941, à l'occasion de la création de l'école supérieure des sciences, le journal Trung Bac Tan Van a publié un article dont l'auteur a souligné: " Notre université de Hanoi a été et sera toujours un service de propagation de la civilisation française en Extrême-Orient ${ }^{34}$ ».

\section{Une œuvre de portée limitée}

En premier lieu, le nombre d'étudiants qui furent formés par l’Université indochinoise était faible par rapport au nombre d'habitants. En 1943-1944, il n’y avait que 1146 étudiants indochinois sur un total près de trente millions d'habitants. Jusqu'en 1945, seuls trois mille étudiants sortirent diplômés de toutes les écoles supérieures en Indochine.

Ensuite, après l'indépendance du Vietnam, le français fut éliminé de l'enseignement général et de l'enseignement supérieur. Dès l'année scolaire 1945-1946, l'université de Hanoi enseignait en vietnamien. Ironiquement, les intellectuels modernes qui avaient eux-mêmes été formés par l'enseignement français (en France ou au Vietnam) eurent un rôle important dans la suppression du français et de l'enseignement français. Hoàng Xuân Hãn, ancien étudiant de grandes écoles (École polytechnique, École des ponts et chaussées, université Paris-Sorbonne), professeur au lycée du protectorat de Hanoi, a publié un vocabulaire scientifique, très important pour l'enseignement supérieur en vietnamien après l'indépendance. Hoàng Xuân Hãn était aussi l'auteur du programme d'étude en vietnamien qui fut appliqué au Vietnam après le 9 mars 1945.

$$
* * *
$$

L'administration française au Vietnam était consciente du rôle de l'enseignement qui visait, d'une part, à la propagation de l'influence culturelle française auprès de la population locale et, d'autre part, à la préparation d'agents auxiliaires des services coloniaux. L'installation et le développement d'un système d'enseignement supérieur occidental, au début $\mathrm{du} \mathrm{xx}^{\mathrm{e}}$ siècle, conduisirent à de grands changements dans l'histoire de l'éducation du Vietnam moderne.

Malgré des avis différents, les chercheurs vietnamiens reconnaissent de plus en plus l'importance de l'Université indochinoise et de l'enseignement supérieur occidental et de son rôle dans les progrès de

34. «Việc lập trường Cao đẳng Khoa học Đông Dương lúc này là rất hợp thời » (« La fondation de l'École supérieure des sciences de l'Indochine est très opportune »), Trung Bắc Tân Văn, nº 76, le 31 août 1941. 
l'enseignement et de l'histoire en général au Vietnam. Les diplômés de l'enseignement supérieur français eux-mêmes, bien que peu nombreux (environ 3000 en tout), contribuèrent grandement à l'indépendance et au développement du pays pendant une longue période. De plus, le modèle français de l'enseignement supérieur perdura à travers le système qui fut mis en place après l'époque coloniale. Après l'indépendance, le gouvernement Hồ Chí Minh a ouvert de nouveau les portes de l'université de Hanoi, dès novembre 1945. Comme témoignage évident de cette filiation, le jour de la réouverture de l'université de Hanoi, le président Hồ Chí Minh délivra des diplômes aux étudiants qui avaient terminé leurs études à l'école de médecine en 1945. Le français perdit son rôle de langue de communication, mais l'enseignement des domaines les plus influencés par l'Occident et qui constituaient de grandes œuvres occidentales, les beaux-arts, les sciences, la médecine notamment, continua après l'indépendance du Vietnam dans la nouvelle organisation. Le programme d'étude des principales écoles supérieures, comme celui de l'école de médecine, des sciences et des beaux-arts, continua à être appliqué à l'Université nationale du Vietnam - nouveau nom de l'Université indochinoise. Cet enseignement garda cette influence française, à travers les professeurs qui avaient été formés par l'enseignement français, en France ou au Vietnam, pendant l'époque précédente: Hồ Đắc Di, Tôn Thất Tùng, Tô Ngoc Vân, Trần Văn Cẩn, Đặng Thai Mai, Nguyễn Mạnh Tường, Cao Xuân Huy, Xuân Diệu, Huy Cận, Nguyễn Văn Huyên, Bùi Kỷ...

Enfin, on peut affirmer que l'université de Hanoi a contribué très largement à l'œuvre culturelle de la France au Vietnam. Elle a contribué à la construction et au développement du système d'enseignement supérieur moderne en jouant un grand rôle dans la transformation et les échanges entre la culture occidentale et orientale au Vietnam. L'université de Hanoi, l'École française d'Extrême-Orient et les instituts Pasteur demeurent les plus beaux symboles culturels et scientifiques de l'influence française pendant toute l'époque coloniale, et jusqu'à nos jours. 



\title{
Sel, soufre et mercure
}

\section{Un multiculturalisme avant la lettre: mémoires d'anciens élèves des lycées français au Sud-Vietnam 1954-1975 \\ Thuy Phuong Nguyen}

\begin{abstract}
Pour les hermétistes, les trois principes : soufre, sel et mercure se retrouvaient dans tous les corps, le soufre, le contenu, se réunissant au sel, le contenant, et au mercure, l'ambiance. Écolier vietnamien dans un lycée français, ne sommes-nous pas un mélange de soufre, de sel et de mercure, le soufre étant le jaune de l'œuf, le sel, sa coquille, et le mercure son blanc, comme l'exprime la composition hermétique de l'ouf ${ }^{1}$ ?
\end{abstract}

\section{Pour une histoire des élèves}

Dans l'Indochine française, le système éducatif colonial se préoccupe d'abord de former les autochtones à des postes subalternes, via des écoles franco-indigènes à l'ambition limitée. Des écoles françaises, similaires à celles de métropole, sont destinées prioritairement aux Européens. Après la Seconde Guerre mondiale, les Français transforment la mission civilisatrice en une diplomatie culturelle chargée de retenir les élites vietnamiennes dans la sphère d'influence française. Sous la colonisation, les élites vietnamiennes parvenaient déjà à mettre leurs enfants dans les lycées français, qui comptaient jusqu'à $20 \%$ d'Asiatiques. À partir de 1945, quand les Français ouvrent librement les portes de leurs

1. Nguyễn Ngọc Châu, "Une période charnière », in Le Temps des Flamboyants, vol. 1, Paris, Amicale des anciens èlèves du lycée Chasseloup-Laubat/Jean-Jacques-Rousseau, 2003, p. 15. 
établissements, les familles vietnamiennes s’y précipitent et le succès est incontestable: de 1954 à 1975, de 20000 à 35000 élèves, dont maintenant $80 \%$ d'Asiatiques, s'y inscrivent chaque année, et l'on peut estimer que 100000 élèves sont passés par ces établissements durant cette période. Il y a alors trois lycées français publics au Sud-Vietnam ${ }^{2}$ - Jean-Jacques Rousseau $^{3}$ (garçons) et Marie Curie (filles) à Saigon, et Yersin (mixte) à Dalat —, qui accueillent environ un tiers de ces élèves, et de très nombreux établissements privés, comme les couvents des oiseaux catholiques ou le grand établissement franco-chinois, le collège Fraternité. Ce sont donc plusieurs générations de Vietnamiens qui sont passés par ces écoles françaises dans le Sud-Vietnam.

Cette jeunesse grandit dans un contexte de bouleversements historiques marqué par les guerres qui se succèdent - Seconde Guerre mondiale, guerre d'Indochine, guerre du Vietnam. Elle assiste à la fin de la colonisation et à la victoire des différentes composantes du nationalisme vietnamien. Elle voit l'influence française diminuer et celle des ÉtatsUnis monter en puissance. Les élèves des lycées français sont nombreux à s'exiler dans les années 1950 et 1960, mais certains restent jusqu'à la réunification en 1975.

Dans le cadre de notre recherche ${ }^{4}$, dont l'une des ambitions était de construire une histoire des élèves ${ }^{5}$ faisant contrepoids à l'histoire d'un système éducatif décrite par les archives, nous avons réuni une centaine de témoignages d'anciens élèves et professeurs de ces établissements et nous avons complété ce corpus par des mémoires publiés, afin de recueillir auprès de ces témoins leur vision des écoles françaises au Vietnam durant cette période. Les questions portaient tant sur le quotidien scolaire que sur leur perception rétrospective de cet enseignement. Comment ces personnes ont-elles vécu cette expérience? Comment ont-elles géré le fait d'apprendre la langue et la culture de l'ancien colonisateur? Que leur a apporté cet enseignement dans leur vie personnelle et professionnelle?

2. Le plus prestigieux lycée français d'Indochine était le lycée Albert-Sarraut à Hanoi. Après 1954, une partie de ses élèves partent avec leurs familles et s'inscrivent dans les lycées du Sud-Vietnam.

3. Anciennement lycée Chasseloup-Laubat, rebaptisé Jean-Jacques-Rousseau en 1955.

4. Nguyễn Thụy Phương, L'école française au Vietnam de 1945 à 1975: de la mission civilisatrice à la diplomatie culturelle, thèse de doctorat de sciences de l'éducation, université Paris Descartes, 2013, 704 p. (dactyl.).

5. Selon l'expression employée par Jean Leduc, François Grèzes-Rueff dans Jean Leduc, François Grèzes-Rueff, Histoire des élèves en France: De l'Ancien Régime à nos jours, Paris, Armand Colin, 2006, 450 p. 
Le texte qui suit est une synthèse de ces témoignages, illustrée par un choix de citations représentatives des aspects identitaires et culturels.

\section{Un quotidien multiculturel}

Les lycées français au Sud-Vietnam sont un lieu d'interactions multiculturelles permanentes. Les élèves se familiarisent d'une part avec la haute culture française des programmes - littérature, philosophie, histoire, arts, voire avec les bonnes manières de la bourgeoisie française -, et d'autre part avec la culture quotidienne au contact de leurs professeurs et camarades français. Extra muros, Américains et Français diffusent, via les mass media, la culture occidentale dans la société vietnamienne. Par ailleurs, les familles mettant leurs enfants dans les écoles françaises sont souvent multiculturelles: franco-vietnamiennes, sino-vietnamiennes, indo-vietnamiennes... Les Eurasiens peuvent être de culture totalement vietnamienne ou totalement française, ou participer des deux cultures:

Je vivais toute mon enfance et adolescence dans une famille vietnamienne, entouré d'oncles, de tantes, de cousins, cousines qui ne me parlaient qu'en vietnamien (ma langue maternelle). J'avais commencé mes classes primaires dans une école vietnamienne avant d'être inscrit dans une école française, donc appris à lire et à écrire le vietnamien avant d'apprendre à lire et à écrire le français ${ }^{6}$.

Dans ce contexte multiculturel, les lycéens des écoles françaises créent leur propre identité entre culture occidentale et culture asiatique:

Les élèves vietnamiens du lycée français, nous nous fréquentons, nous avons les mêmes loisirs: le cinéma occidental, les lectures des livres et de bandes dessinés en français. De plus, nos familles avaient en gros les mêmes modes de vie. C'est grâce à la culture familiale que nous gardons notre culture orientale: respect envers les ancêtres et les grandes personnes, ordre des préséances ${ }^{7} \ldots$

Avec l'arrivée de Ngô Đình Diệm au pouvoir en 1955, le contexte politique devient hostile à la France, à qui les nationalistes Sud-vietnamiens reprochent à la fois des attitudes néocoloniales et un neutralisme vis-à-vis du Nord-Vietnam. La langue vietnamienne retrouve sa place

6. Témoignage de Imre Szabo (Albert-Sarraut et Chasseloup-Laubat, 1955). Pour les notes de bas de page concernant les témoignages d'anciens élèves, la parenthèse qui suit le nom du témoin indique son ou ses établissements d'origine ainsi que l'année d'obtention de son baccalauréat.

7. Témoignage de Ngô Trí Hùng (Albert-Sarraut et Jean-Jacques-Rousseau, 1962). 
dans l'espace public, notamment dans l'enseignement, au détriment du français. Sous la colonisation, il était parfois interdit aux élèves de parler vietnamien entre eux dans les écoles françaises ${ }^{8}$, une règle que perpétuent certains établissements privés jusqu'aux années 1950:

Avant le gouvernement de Ngô Đình Diệm, il était interdit, au couvent des oiseaux, de parler vietnamien même dans la vie quotidienne, parce que c'est le milieu de la haute société vietnamienne et de la noblesse française, les sœurs en grande majorité en sont issues. Si nous parlions vietnamien par mégarde, nous subissions des punitions ${ }^{9}$.

Dans les lycées français publics, cependant, les élèves peuvent librement converser en vietnamien depuis la fin de la colonisation, tandis que les élèves de familles francophiles s'expriment toujours en français s’ils le veulent:

Entre nous, à la récréation et à la maison, nous ne parlions jamais français. Nous le parlions seulement en classe pour répondre au professeur ${ }^{10}$.

Certains d'entre nous parlaient plus le français que le vietnamien dans la cour de récréation. J'en faisais partie, et je le revendique ${ }^{11}$.

En dépit de l'hostilité ambiante, la mission d'enseignement français et de coopération culturelle ${ }^{12}$ profite de l'attirance ininterrompue des élites vietnamiennes pour la culture française pour faciliter, par exemple, la diffusion des livres, ce qui permet aux jeunes d’y accéder à coût réduit:

En ces années [19]50 et [19]60, un accord culturel franco-vietnamien permettait aux livres, magazines et illustrés français d'être vendus au Vietnam du Sud à des prix fondés sur un taux de 10 piastres pour $100 \mathrm{AF}$ puis $1 \mathrm{NF}$. Imaginez ma joie de pouvoir m'acheter un livre de poche pour 10 ou 15 piastres selon sa pagination, chez Khai Tri ou Xuân Thu, ou chez Lê Phan, quand ma mère me récompensait ${ }^{13}$ !

8. Témoignage de Maurice Lavocat-Dubuis (Albert-Sarraut, 1952).

9. Témoignage de Nguyển Thị Lan Hương (école Sainte-Marie et couvent des oiseaux, 1961).

10. Témoignage de Đinh Trọng Hiếu (Albert-Sarraut, Yersin et Jean-Jacques-Rousseau, 1956).

11. Georges Nguyễn Cao Đức, "Mon vieux lycée, que de choses ne te devons-nous pas... », in Le Temps des Flamboyants, vol. 2, Paris, Amicale des anciens èlèves du lycée Chasseloup-Laubat/ Jean-Jacques-Rousseau, 2005, p. 9.

12. Cette mission est créée en 1953 pour prendre en charge l'ensemble des actions culturelles de la France en Indochine. Elle dépend d'abord du commissariat général de France en Indochine, passe sous la tutelle du ministère des Affaires étrangères en 1955 et devient en 1958 le service culturel de l'ambassade de France à Saigon.

13. Georges Nguyễn Cao Đức, « Ma vie de lycéen, l'argent, et moi », in Le Temps des Flamboyants, p. 71. 
La langue et la littérature française sont appréciées des élèves pour des raisons diverses: tradition familiale, prestige de la culture française, esthétique, divertissement, voire intérêt pour la politique:

C'était mon père qui - ayant reçu une éducation française sous l'influence du colonialisme - m'a initiée, dès l'enfance, au français par des cassettes de chansons enfantines, des contes, des livres illustrés... Féru de littérature française, fervent lecteur, capable de réciter textes et poèmes de certains écrivains par cœur, mon père nous a immergés de bonne heure dans cette atmosphère poétique ${ }^{14}$.

En grandissant, avec les cours de grammaire, de conjugaison, les poésies, j'ai découvert la beauté de cette langue, la rigueur, la précision de ses mots [...]. La littérature française me passionnait, plus particulièrement la période du xixe siècle: le romantisme, les romanciers comme Balzac, Zola, qui décrivent la société et l'âme humaine.

Le lycée à l'époque c'était aussi... les échanges de bandes dessinées, la découverte du monde grâce aux « Histoires de l'oncle Paul » du journal Spirou, les livres de la Bibliothèque verte ${ }^{15}$.

Les premiers livres que j’ai empruntés [dans les bibliothèques françaises] ont été Le Manifeste du parti communiste de Karl Marx, interdit et introuvable à l'époque et on ne le trouvait nulle part ailleurs, et Félicité Robert de Lamennais ${ }^{16}$.

Cette vision enthousiaste doit cependant être nuancée. La majorité des élèves ne sont pas francophones, ce qui pose des problèmes de niveau scolaire qui n'existaient pas dans la période coloniale. Pour ces élèves, la langue française est un obstacle souvent difficile de surmonter:

Quand quelqu'un apprend le français sans initiation préalable, sans culture générale et sans tempérament littéraire, les résultats ne peuvent être des plus enthousiasmants. C'était le cas de la plupart d'entre nous à l'époque. [...] En littérature, on savait qui faisait quoi dans L'Avare, Le Cid, les Fables de La Fontaine, ce qui nous fournissait quelques arguments valables dans nos dissertations qui restaient maigres malgré nos efforts. Les efforts justement franchissaient mal le vocabulaire, l'expression, le regroupement des idées et le style ${ }^{17}$.

14. Minh Châu, "À l'ombre des filaos, le collège français de Nha Trang 》, in Livre de souvenir, 2008. Texte publié sur le site web des anciens élèves du collège français de Nha Trang : http:// collegefrancaisnhatrang.net/livre/html/Nha\%20Trang\%20va\%20toi\%20-\%20MChau\%201.html 15. Nguyễn Ngọc Châu, Une période charnière, p. 14.

16. Témoignage de Đinh Trọng Hiếu (Albert-Sarraut, Yersin et Jean-Jacques-Rousseau, 1956).

17. Témoignage de Lê Quan Thanh (Chasseloup-Laubat, 1954). 
Par ailleurs, la littérature asiatique reste importante aux yeux des élèves, qu'il s'agisse des classiques vietnamiens ou, surtout, des romans de chevalerie chinois traduits en vietnamien, qui envahissent le marché en 1962, et dont la mauvaise qualité provoque l'inquiétude des familles et des éducateurs ${ }^{18}$.

Comme la plupart des jeunes Vietnamiens de mon temps, je lisais souvent des fables, récits, histoires et poèmes de la langue vietnamienne, surtout les auteurs renommés tels que Nguyễn Du, Hồ Xuân Hương, Nguyễn Trãi ${ }^{19}$ etc. $^{20}$

[Les lycéens] étaient nombreux à dévorer les «truyện chưởng ", ces romans-fleuves de douze à treize tomes de quelques cinq cents pages chacun, loués à la sauvette et passés par des centaines de mains donc bourrés de microbes ${ }^{21}$ !

Ce multiculturalisme du goût se retrouve aussi dans la musique. Les chanteurs préférés des lycéens sont Français, Américains, Britanniques et Vietnamiens. Le répertoire des artistes locaux est souvent multilingue. Comme en Occident, l'arrivée des Beatles et de la musique populaire anglo-saxonne enflamme les lycéens, ce qui n'empêche pas certains d'être attachés à des formes plus traditionnelles, comme l'opéra rénové cải lương ${ }^{22}$ :

Deux camps complémentaires et interpénétrables chez les élèves: on pouvait savourer Georges Guétary, Sylvie Vartan, Dalida, tout en appréciant Ban Thăng Long, Thanh Thúy, Khánh Ly ${ }^{23}$ etc.

The Beatles, Woodstock, le mouvement Yé-yé, la vague déferlante jusqu'à notre petite province... Mon père a été choqué dès la sortie des posters qui ont commencé à gagner nos murs: notre chambre entièrement tapissée de photos de groupes de chanteurs rock et ce que les passants ont pu apercevoir de la rue, il y avait de quoi le contrarier: ses filles complètement dingues de ces idoles aux cheveux longs ${ }^{24}$ !

18. Stanley A. Barnett, Erroll D. Michener, C. Walter Stone, Developmental book activities and needs in the Republic of Vietnam, Wolf Management Services/Contract No. AID/csd-1162, octobre 1966, p. 63.

19. Nguyễn Du (1766-1820) : auteur du célèbre roman-poème L'histoire de Kiều; Hồ Xuân Hương (1772-1822) : poétesse; Nguyễn Trãi (1380-1442) : homme d'état et poète.

20. Témoignage de Nguyễn Xuân Phong (Chasseloup-Laubat, 1953).

21. Bích Diệp, «Les cinémas », in Livre de souvenir, 2008. Texte publié sur le site du collège français de Nha Trang: http://collegefrancaisnhatrang.net/livre/html/cinema.html

22. Forme d'opéra vietnamien, à la mode depuis les années 1930. Georges Nguyễn Cao Đức, "La vie des élèves hors du lycée dans les années 50-60 », in Le lycée Chasseloup-Laubat/ Jean-Jacques Rousseau et son temps (CD-Rom), Paris, Amicale des anciens èlèves du lycée Chasseloup-Laubat/Jean-Jacques-Rousseau, 2007.

23. Ban Hợp Ca Thăng Long : groupe vocal qui connait un grand succès jusqu'en 1975 ; Thanh Thúy et Khánh Ly: chanteuses populaires au Sud-Vietnam.

24. Minh Châu, « À l'ombre des filaos, le collège Français de Nha Trang ». 
Malgré cette éducation « à la française ", j’ai toujours aimé — ô paradoxe extrême! — le cải lương [...]. Eh bien oui, en ce temps-là, j’adorais en même temps et la voix rauque de Johnny chantant " Le pénitencier " et la voix feutrée et "sucrée " de Thành Được se lamentant dans un vọng cổ (littéralement "penser avec admiration, regret, au passé »: forme de chanson triste) langoureux, l'amour perdu d'un étudiant pauvre regardant, impuissant, sa dulcinée se marier avec le richard du village ${ }^{25}$ !

Certains lycéens se lancent avec succès dans une carrière musicale, comme l'auteur-compositeur-interprète engagé Trịnh Công Sơn, qui devient rapidement une figure majeure de la chanson vietnamienne, la chanteuse et actrice Thanh Lan, au répertoire français, anglais et vietnamien $^{26}$, ou encore Elvis Phương et Lê Hựu Hà, pionniers du rock vietnamien qui trouvent une audience chez les soldats américains ${ }^{27}$.

En matière de cinéma, les goûts des élèves vont d'abord aux films français et américains, plutôt qu'aux productions chinoises et indiennes. Le cinéma est un de leurs loisirs favoris, et le choix est vaste: dans les années 1970, Saigon ne compte pas moins de soixante-dix salles ${ }^{28}$ :

Rappelez-vous aussi ces cinémas Dai Nam, Rex qui passaient les grandes productions hollywoodiennes comme Ben Hur, Quo Vadis, les salles Vinh Loi, Le Loi, des permanentes où on regardait en boucle les films avec Alan Ladd, Robert Mitchum ou Randolph Scott. [...] Et bien sûr, il y avait le cinéclub où on regardait en V.O. grommeler Jean Gabin, Pierre Brasseur ${ }^{29}$.

Les stars occidentales et les superproductions ne sont pas les seules raisons d'aller au cinéma. Les films doublés en français et sous-titrés en vietnamien ${ }^{30}$ permettent de se perfectionner en français « que ce soit par la bouche d'Alan Ladd, Alain Delon, Gary Cooper, Brigitte Bardot ou de celle de Jean Gabin ${ }^{31}$ ". À la fin des années 1960, les lycéens vont dans les

25. Võ Thành Thọ, « J'aime écouter le « cải lương », in Le Temps des Flamboyants, p. 54.

26. Hoàng Đình Tuyên, "Chansons françaises interprétées par Thanh Lan MC 67 et Elvis Phuong JJR 64 », in Magazine Good Morning, septembre 2011, $n^{\circ}$ 125. II s'agit du magazine mensuel créé et publié en ligne par les anciens élèves du lycée Chasseloup-Laubat/JeanJacques Rousseau : http://aejjrsite.free.fr/goodmorning

27. Trần Đăng Chí, « Memoir ». Texte publié sur le blog personnel de Trần Đăng Chí (Institution Taberd, Saigon, 1965) : http://chitranphotography.blogspot.fr/2003_11_01_archive.html.

28. Trần Đăng Chí, « Các rạp xi-nê Sài Gòn trước 1975 ("Cinémas de Saigon avant 1975 ») », in Magazine Good Morning, n 89, septembre 2008.

29. Đỗ Trịnh Kỳ, « Les élucubrations d'un ancien élève de JJR », in Le Temps des Flamboyants, p. 88. 30. Georges Nguyễn Cao Đức, " La vie des élèves hors du lycée dans les années cinquante-60 », in CD-Rom.

31. Ibid., in CD-Rom. 
salles fréquentées par les soldats américains pour améliorer leur anglais ${ }^{32}$. Les films étrangers sont aussi une ouverture sur le monde extérieur, et les écoles françaises organisent des projections, soit dans l'établissement même, soit dans les centres culturels français ou dans des salles louées:

Un soir, après avoir vu le film « La Strada » [...], mes larmes tombèrent silencieusement dans mon plat et se mélangèrent à la bonne soupe que m’avait préparée le cuisinier. Je sus alors que la vie, la vraie vie n'était pas celle qui m'apparaissait dans toute la quiétude d'un petit garçon, fils de ministre saïgonnais ${ }^{33}$.

Comment oublier une projection sur Van Gogh en noir et blanc, alors oublié des lycées et des boutiquiers, où le maître faisait l'éloge de la folie et du délire, du drame et de la passion. L'introduction du cinéma dans l'éducation du regard et de l'écoute était chose prodigieusement nouvelle ${ }^{34}$.

Outre le cinéma, les élèves ont un vaste choix d'activités périscolaires — natation, athlétisme, gymnastique, escrime, tennis, sports collectifs qu'ils pratiquent au lycée ou dans les clubs sportifs. Ces activités sont très populaires et les professeurs de sport sont bien présents dans les souvenirs ${ }^{35}$. Le scoutisme est très apprécié, mais la guerre du Vietnam met fin aux sorties en plein-air au milieu des années $1960^{36}$. À ces activités très occidentales s'ajoutent des jeux spontanés où cultures enfantines française et vietnamienne se mélangent. Comme des enfants français, ils jouent à colin-maillard, aux billes, aux toupies ${ }^{37}$, au ballon prisonnier ${ }^{38}$, mais, comme des enfants vietnamiens, ils s'envoient des boulettes de caoutchouc faites avec le latex des hévéas des cours de récréation, font des parties de đá cầu (jeu de volant qui se joue avec le pied) et organisent des combats de grillons achetés pour quelques đông chez les marchands ambulants $^{39}$.

En matière de mode, les élèves sont à peu près libres de s'habiller et de se coiffer comme dans les magazines et les films importés d'Occident,

32. Trần Đăng Chí, Các rạp xi-nê Sài Gòn trước 1975 (« Cinémas de Saigon avant 1975 »).

33. René Nguyễn Dương Liên, «Ô Doux Aventin de ma jeunesse », in Magazine Good Morning, no 87 , juillet 2008.

34. Trịnh Nghĩa Trinh, « Le professeur Pierre Ansart et moi », in Le Temps des Flamboyants, p. 26.

35. Georges Nguyễn Cao Đức, "La vie des élèves hors du lycée dans les années 50-60 », in CD-Rom.

36. Ibid., in CD-Rom.

37. Đỗ Trịnh Kỳ, Les élucubrations d'un ancien élève de JJR, p. 89.

38. Trần Ngân Diệp, «La balle au prisonnier », in Le Temps des Flamboyants, vol. 1, p. 113.

39. Nguyễn Bá Đàm, « Combats de grillons », in Le Temps des Flamboyants, vol. 2, p. 60. 
une liberté qui les distingue de leurs camarades en uniforme des écoles privées et des écoles vietnamiennes:

Mon copain, leader de la bande, était " gay " et n'était que mon conseiller de mode! Ces jeunes gens étaient encore plus coquets que nous, les filles, et s'habillaient toujours de façon "Fashion ", esclaves de la mode: chemises en mousseline ou en soie $^{40}$ !

Ce doit être vers le début des années [19] 60, à l'époque des “ Demi-sel » ${ }^{41}$, le film culte des «vilains garçons » avec Horst Buchholz comme idole, tout de cuir noir vêtu. Côté filles, la jupe serrée à la BB commençait à faire des ravages au lycée. Cela tranchait singulièrement des tuniques longues des filles de Bùi Thi Xuân ou des jupes plissées bleues des « couventines " de chez les sœurs du couvent des Oiseaux ${ }^{42}$.

Comme en France, leurs goûts vestimentaires sont mal vus des familles et des autorités scolaires. Au lycée Marie-Curie et au couvent des oiseaux, les surveillantes vérifient chaque lundi que les jupes ont une longueur décente. D’ailleurs, même dans les écoles vietnamiennes, on vérifie que les pans du áo dài ${ }^{43}$, qui ont aussi tendance à raccourcir, dépassent d'au moins 10 centimètres au-dessous du genou ${ }^{44}$ :

La mode des minijupes n'a pas trouvé grâce aux yeux de mon père et chaque jour, avant d'aller à l'école, j'ai toujours eu droit à une inspection générale de la tenue, comme à l'armée! Mais les pattes d'éléphant en pantalons ne l'ont point dérangé! Il faut dire que ma fantaisie vestimentaire inspirée des hippies lui a fait pousser souvent des cris de désespoir ${ }^{45}$ !

Avec Monsieur Lam les jupes n'étaient jamais assez longues alors que pour vous, elles n'étaient jamais assez courtes. Quant aux garçons, les dimensions réglementaires étaient inversées, mais cette fois pour les cheveux; toujours trop courts pour vous et trop longs pour Monsieur Lam ${ }^{46}$.

40. Tiên Sa, « Les malheurs de Sophie (alias Tien Sa)», in Livre de souvenir, 2008 : http://collegefrancaisnhatrang.net/html/les\%20malheurs\%20de\%20Sophie.html

41. Titre français du film allemand Die Halbstarken (1956).

42. Bùi Thị Xuân est une école publique vietnamienne. Vinh Sang, « Lettre de décembre 2001 », in Entre nous Yersiniens, décembre 2001. II s'agit d'un des sites des anciens élèves du lycée Yersin : http://entre-nous.perso.neuf.fr/2001_12.html

43. Le áo dài, cette élégante tunique féminine qui symbolise souvent la culture vietnamienne, a été créée en 1934 par un couturier vietnamien formé à la mode française.

44. Huỳnh Bội Trân, Vietnamese aesthetics from 1925 onwards, thèse de doctorat des arts visuels (PhD in Visual Arts), University of Sydney, Sydney College of the Arts, 2005, 418 p. (dactyl.), p. 205.

45. Minh Châu, Nha Trang et moi.

46. Michel Brun, "Mes chers amis », in Bulletin de l'association Fraternité de Saigon-Cholon, $n^{\circ} 31$, janvier 2011. Texte publié sur le site de l'Association Fraternité de Saigon - Cholon (Bác Ái): http://bacai.free.fr/bulletin31.htm 
L'habillement peut aussi avoir une signification politique. Pendant la guerre d'Indochine, puis pendant la guerre du Vietnam, des lycéennes de Marie-Curie revêtent un áo dài blanc plutôt que la jupe pour signifier leur nationalisme:

J'étais la seule à porter le áo dài dans un lycée français. On m’a remarquée pour cela mais je n'étais ni ridiculisée ni embarrassée par cette tenue. Ça, c'est l'avantage d'un environnement de liberté, on respecte votre choix sans le juger ${ }^{47}$.

C'est en matière de cuisine que la culture asiatique - à peine concurrencée par le Coca-Cola, le chocolat et les glaces - domine le plus le quotidien des élèves. Friandises, saveurs, odeurs, cris des vendeurs ambulants et repas familiaux sont gravés dans la mémoire des anciens comme autant de madeleines proustiennes:

Il y avait tous ces marchands ambulants qui proposaient des plats (bánh cuốn, bò bía, hủ tiếu, mì), des fruits, certains marinés dans du jus de réglisse (cóc, ổi, tầm ruột), des boissons ${ }^{48}$...

Nous mangions souvent du ragoût, pratique pour une grande famille. Nous en mangions avec des patates et du riz, c'était très bon surtout rajouté avec des piments. Un autre plat prisé de nous tous c'était évidemment, le carry poulet. Un autre plat exceptionnel était l'igname en sauce ${ }^{49}$ :

En revanche, les cantines scolaires françaises n'ont pas laissé de bons souvenirs, comme au couvent des Oiseaux:

L'école nous servait un plat appelé « jardinière » tous les dimanches soir. [...] Nous détestions ce plat à cause de son manque de goût, particulièrement après avoir passé notre dimanche en ville à manger nos plats vietnamiens favoris, tels que le phở ou le bánh xèo ${ }^{50}$.

Les nonnes finissent par faire accepter cette jardinière aux jeunes filles en leur racontant que les Françaises ont les yeux bleus "parce qu'elles mangent beaucoup de carottes "...

\footnotetext{
47. Témoignage de Bùi Trân Phượng (Marie-Curie, 1968).

48. Đỗ Trịnh Kỳ, Les élucubrations d'un ancien élève de JJR.

49. II s'agit d'une famille indo-vietnamienne. R. Beaumont, «René se souvient: Vietnam mes jeunes années », in Rue Calmette, de l'Indochine à Paris. Ce blog de la famille Beaumont est consacré aux histoires personnelles des membres de cette famille: http://intersto.free.fr/?p=84

50. Lucy Nguyen Hong Nhiem, A Dragon Child: Reflection of a Daughter of Annam in America, Bloomington, iUniverse, 2004, p. 37.
} 


\section{Entre deux cultures}

Cette immersion adolescente dans le bain multiculturel du SudVietnam d'après 1954 a fait des élèves des passeurs entre Orient et Occident. Les anciens lycéens ont une vision positive de leur double culture, et revendiquent leur passage par l'école française comme un élément fondamental de leur identité actuelle:

Je pense que les années passées au lycée ont fait de moi, comme de nous tous, un point de rencontre de deux cultures. La première était celle dont nous étions naturellement imprégnés et qui était transmise au jour le jour par notre entourage. La seconde nous était parvenue par les livres, l'éducation, les explications de nos instituteurs et de nos professeurs ${ }^{51}$.

Je considère la culture vietnamienne comme un fleuve avec les cultures française, chinoise et américaine comme affluents qui enrichissent mon identité $^{52}$.

Bien que je m'approprie parfaitement la culture française, je me suis forgé une identité vietnamienne. Ma culture vietnamienne est représentée par les coutumes et pratiques que je respecte dans la vie courante: cérémonies d'enterrement, anniversaire de la mort des ancêtres, fêtes traditionnelles... Ce qui est très français chez moi est le raisonnement, les réflexions, je m'exclame souvent en français bien que j'aie toujours vécu au Vietnam [...]. J’ai été parfaitement imprégné de culture française dès mon plus jeune âge, il est impossible de la purger ${ }^{53}$.

Ce biculturalisme, s'il est bien assumé aujourd'hui, n’a pas toujours été simple à vivre. D’une part, le contexte politique fait que les Vietnamiens des lycées français ont maille à partir avec les élèves des lycées nationaux, qui les accusent d'être des việt gian, traîtres à la patrie, lors de batailles de rue, ou, de façon plus policée, par voie de presse, comme dans le texte suivant, extrait d'une polémique qui oppose en 1970 lycées français et vietnamiens dans le magazine pour la jeunesse Thằng Bờm ${ }^{54}$ :

Vous n’avez pas été élevé au pain et au lait de vache, mais au riz, à la sauce de poisson, et avec l'amour de vos parents vietnamiens. Ne soyez pas infectés par les coutumes étrangères, ne copiez pas le mode de vie buissonnier américain et occidental en portant des vêtements ridicules, en vous

51. Nguyễn Ngọc Châu, Une période charnière.

52. Témoignage de Nguyễn Lê Hiếu (Albert-Sarraut et Jean-Jacques Rousseau, 1957).

53. Témoignage de Nguyễn Xuân Thu (Albert-Sarraut, 1952).

54. Cet épisode est rapporté en détail dans un article d'Olga Dror. Olga Dror, «Raising Vietnamese: War and Youth in the South in the early 1970s », Journal of Southeast Asian Studies, vol. 44, no 1, 2013, p. 74-99. 
comportant avec mauvaise foi, et en nuisant à la nation vietnamienne! [...] Votre éducation est du genre pour colonialistes et, en outre, elle est complètement stupide. [...] Pourquoi adhérez-vous encore à quelque chose qu'après quatre-vingts ans de domination notre peuple n’a plus accepté?

Face aux accusations de leurs camarades des lycées nationaux, les élèves des lycées français affirment être de vrais Vietnamiens, même si certains lycéens s’interrogent en privé sur leur " enrôlement dans cet enseignement aux relents désuets de colonialisme ${ }^{55}$ ":

Nous avons fondé un comité entre plusieurs lycées français au Vietnam pour faire une note expliquant aux élèves des écoles vietnamiennes que nous n'étions pas des "việt gian ". [...] Nous apprenons la langue et la culture française mais nous ne sommes pas favorables aux politiques de la France ${ }^{56}$.

À un niveau plus personnel, le conflit identitaire, quand il existe, se révèle progressivement, et parfois rétrospectivement, avec la maturité:

On vivait simultanément dans deux mondes très différents sans pour autant sentir de déchirement ou conflit. Est-ce parce qu’on était trop jeune pour s'apercevoir des contradictions? Ou est-ce que ces deux mondes avaient été disposés dans des compartiments différents ${ }^{57}$ ?

Une fois devenu lycéen, conscient de notre environnement, de la situation du pays, nous commencions à prendre conscience des différences de deux cultures occidentales et orientales. [...] Une fois devenu adulte et autonome, avec un regard rétrospectif, nous avons conscience de l'écartèlement, du déchirement que nous avons vécu ${ }^{58}$.

Certains élèves souffrent du conflit entre les valeurs de liberté portées par l'enseignement français et le respect confucéen des normes et hiérarchies sociales:

Notre mère considérait son fils de 18 ans comme un enfant et non comme un adulte et elle n'hésitait pas à le frapper devant ses petits frères pour le faire obéir. Les bacheliers vivaient donc une contradiction entre le concept théorique de liberté tel qu'il était enseigné à l'école et la réalité d'une société encadrée, contrainte et normée $e^{59}$.

55. Đỗ Khiêm, " Je préfère la langue de la Commune à celle des Versaillais », Courrier international, n 802, 16 mars 2006.

56. Témoignage de Phạm Ngọc Tuấn (Jean-Jacques Rousseau, 1960).

57. Témoignage de Marie Paule Ha (école Sainte-Thérèse et couvent des oiseaux, 1969).

58. Témoignage de Ngô Trí Hùng (Albert-Sarraut et Jean-Jacques-Rousseau, 1962).

59. Témoignage de Hoàng Văn Nam (Blaise-Pascal et Yersin, 1959). 
Ce conflit est particulièrement sensible pour les jeunes filles. Ainsi, une lycéenne choque sa famille quand elle leur dit vouloir choisir ellemême son époux:

"Comment ose-t-elle, notre petite fille, prendre cette décision sans rien nous demander? " Ma mère pleurait et blâmait notre père de m'avoir envoyée à l'école française. "Ta fille a sans doute lu trop de romans d'amour à l'école ${ }^{60}$. "

Une fois mariée, elle s'aperçoit que sa scolarité française ne l'a pas préparée à son rôle d'épouse confucéenne, consistant à " accomplir des tâches de domestique dans ma propre famille ${ }^{61}$ ». Parfois, seul le départ à l'étranger permet de résoudre le conflit:

Une partie d'entre nous, comme moi, vivaient en respectant toutes les coutumes traditionnelles, surtout dans le cadre familial. C'est vrai qu'on vivait dans les contradictions, je sentais que j'avais deux personnes en moi. [...] Une fois que je me suis installée au Canada à l'âge de 25 ans, à ce moment-là, je me suis posé la question de savoir si je devais vivre librement pour moi-même ou je devais vivre pour mes parents ${ }^{62}$.

Certains élèves restent profondément attachés à leur culture d’origine dans ses différentes dimensions intellectuelles, morales et familiales, et travaillent à la conserver :

Pendant ma scolarité à l'école française, je ne voulais pas être appelé thằng Tây con [gosse francisé], donc assimilé. J'ai toujours eu envie de mieux maîtriser la culture et la littérature vietnamienne parce que je ne voulais pas être dépassé en matière de connaissances sur l'histoire et la culture du pays par mes amis scolarisés à l'école vietnamienne ${ }^{63}$.

Les racines se vivent, vivent, et se (re) découvrent, car nul ne peut se renier, on est ce que l'on est. Pour preuve paradoxale et dans mon seul cas, ma demande à mes parents - pas si étonnés que cela - de m'envoyer des ouvrages du groupe littéraire Tự Lực văn đoàn ${ }^{64}$, à peine arrivé en France en 1965. Et ce cas fut majoritaire ${ }^{65}$.

60. Lucy Nguyen Hong Nhiem, A Dragon Child, p. 44.

61. Ibid., p. 47.

62. Témoignage de Nguyễn Thị Xuân Mai (Marie-Curie, 1968).

63. Témoignage de Võ Thành Thọ (Jean-Jacques-Rousseau, 1968).

64. Tự Lực văn đoàn [Groupe littéraire autonome] fondé en 1934, partisan de la modernité. Nguyễn Văn Ký, La société vietnamienne face à la modernité, Paris, L'Harmattan, 1995, p. 105-109.

65. Georges Nguyễn Cao Đức, Mon vieux lycée, que de choses ne te devons-nous pas..., p. 10. 
J'ai une double culture, je pense français, je raisonne en français, je suis cultivée en français mais je vis vietnamien selon les principes du nền nếp gia phong [discipline et ordre au sein de la famille], et pour les femmes du công, dung, ngôn, hạnh [travail domestique, beauté, politesse et bonne conduite]. Je ne perds jamais l'âme vietnamienne ${ }^{66}$.

Malgré ces efforts, la perte des repères culturels suite à l'exil est souvent déplorée, notamment chez les Eurasiens et les naturalisés.

À l'heure actuelle, j'écris en français encore mieux qu'en vietnamien. Je ne regrette qu'une seule chose, c'est que je ne me sens pas pleinement vietnamien puisque je ne maîtrise pas bien l'histoire du Vietnam, c'est un trou difficilement comblé. C'est mon point faible, ce qui suscite en moi un complexe culturel ${ }^{67}$.

De nationalité française, nous n'avions pas eu d'enseignement en vietnamien, si bien que nous ne savons pas lire, encore moins écrire le viêt. La littérature étant dans un registre soutenu, cela nous avait fermés complètement de cette culture ${ }^{68}$.

\section{La base initiale d'une vie}

Les témoins affirment que l'enseignement français leur a donné une base à la fois pratique, intellectuelle et morale pour leur vie personnelle et professionnelle. L'apport de connaissances scolaires est perçu comme secondaire par rapport à la transmission d'une culture générale, de méthodes de raisonnement et d'acquis plus personnels comme la curiosité ou la capacité d'adaptation:

Que nous a offert finalement notre lycée, avec le recul du temps? La réponse est fort simple: la base initiale d'une vie ${ }^{69}$.

L'enseignement français apporte plus une culture générale que des connaissances, comme le dit la phrase «la culture c'est ce qui reste quand on a tout oublié ", et fait de nous des " gentilshommes " plutôt que des « savants " ${ }^{70}$.

Nous sommes reconnaissants à l'éducation française dont nous bénéficions, elle nous forme un jugement cartésien, nous transmet la curiosité,

66. Témoignage de Nguyễn Thị Lan Hương (école Sainte-Marie et couvent des oiseaux, 1961).

67. Témoignage de Nguyễn Xuân Thu (Albert-Sarraut, 1952).

68. Témoignage de Solange Ly (Marie-Curie et couvent des oiseaux, 1971).

69. Georges Nguyễn Cao Đức, Mon vieux lycée, que de choses ne te devons-nous pas..., p. 10.

70. Témoignage de Nguyễn Đốc Khanh (Albert-Sarraut et Jean-Jacques Rousseau, 1957). 
nous offre une capacité d'adaptation, nous apporte le désir de se battre pour réussir ${ }^{71}$.

Le fait de savoir et de comprendre débarrasse les anciens colonisés de leur complexe d'infériorité:

Je n’ai pas de complexe d'infériorité mais un complexe de "supériorité " car nous connaissons largement et profondément des choses, de façon diversifiée, nous sommes formés de façon complète par l'enseignement français $^{72}$.

Les valeurs phares portées par l'enseignement français sont l'humanisme, la liberté et l'égalité - tout ce que les Français craignaient tant de voir transmettre aux élèves sous la colonisation - et certains anciens les comparent en positif aux valeurs confucéennes:

L'école française [...] m’a offert un véritable humanisme, une pensée et un comportement humanistes ${ }^{73}$.

La culture française, avec sa richesse élégante, est bien une source d'inspiration pour mes activités scientifiques. La largesse d'esprit qui s'ouvre à tout ce qui est humain ${ }^{74}$.

L'enseignement français est une porte d'ouverture pour les Vietnamiens de culture extrême-orientale avec ses valeurs (hiérarchie, justice, obéissance...) vers de nouvelles notions de la civilisation occidentale (liberté, démocratie, indépendance...), donc une porte ouverte vers la modernité internationale via la langue française et l'enseignement français ${ }^{75}$

Les concepts de liberté et d'égalité sont transmis notamment par l'attitude des enseignants, qui encouragent les élèves à l'autonomie et au sens critique:

Mes professeurs ont su me laisser le libre arbitre en m'offrant un large choix. Du coup, une fois adulte, je me suis engagé politiquement, mais je n'ai jamais pu, ni voulu, m'engager dans un parti politique quelconque. [...] Ce sont bien les professeurs de Jean-Jacques Rousseau qui m'ont appris à apprendre ${ }^{76}$.

71. Témoignages de Phạm Ngọc Tuấn (Jean-Jacques-Rousseau, 1960), Ngô Tri Hùng (JeanJacques-Rousseau, 1962) et Nguyễn Thị Xuân Mai (Marie-Curie, 1968).

72. Témoignage de Nguyễn Văn Lộc (Jean-Jacques Rousseau, 1968).

73. Témoignage de Nguyễn Xuân Hồng (Yersin et Jean-Jacques-Rousseau, 1960).

74. Témoignage de Phạm Gia Khải (Albert-Sarraut, 1954).

75. Témoignage de Hoàng Văn Nam (Blaise-Pascal et Yersin, 1959).

76. Đặng Đình Cung, «Ce que je dois à mon lycée », in Le Temps des Flamboyants, vol. 1, p. 32. 
Cinquante ans après, j'essaie d'analyser les ingrédients de ce bouleversement. D’abord et surtout, l'apprentissage de l'esprit critique. Pour la première fois, l'on avait l'impression de penser par soi-même et l'on découvrait émerveillé l'intrusion du subjectivisme dans notre conscience, l'individualisation du regard, la conquête de la liberté, le mépris ironique des corporations, des corps constitués, de la convention ${ }^{77}$.

Les innovations pédagogiques, le choix éclectique d'auteurs contemporains, l'ouverture à d'autres formes culturelles et surtout l'encouragement à l'initiative intellectuelle et au débat contradictoire, ont été pour ces élèves une véritable leçon de vie, un apprentissage de la liberté personnelle qui les a aidés à traverser une époque difficile tant dans l'histoire de leur pays que dans leur histoire personnelle.

$$
* * *
$$

Les lycées français du Sud-Vietnam sont des lieux où débute un processus d'acculturation individuelle tel que l'a décrit le psychologue John W. Berry.

$\mathrm{Au}$ niveau individuel, il faut considérer les changements psychologiques auxquels sont soumis les individus et les effets de leur adaptation finale à leur nouvelle situation. Ces changements peuvent constituer un ensemble de changements comportementaux qui s'effectuent aisément (dans les façons de parler, de manger ou de s'habiller; dans l'identité culturelle) ou ils peuvent être plus problématiques, en produisant un stress d'acculturation qui se manifeste par de l'incertitude, de l'anxiété et de la dépression ${ }^{78}$.

Dans le cas qui nous intéresse, le stress d'acculturation dont parle Berry est évoqué par les témoins, qui ont dû apprendre très jeunes à naviguer, plus ou moins heureusement, entre deux philosophies sociales souvent opposées - «Voltaire contre Confucius ", selon la formule de l'écrivain Đỗ Khiêm ${ }^{79}$ - avec ce que cela entraîne d'angoisses et de tensions familiales. Certains témoins ont aussi l'impression d'avoir perdu un peu de la partie vietnamienne d'eux-mêmes, voire d'en avoir été privés, dans le cas des Eurasiens et des naturalisés.

77. Trịnh Nghĩa Trinh, Le professeur Pierre Ansart et moi.

78. John W. Berry, «Introduction: social change and acculturation », in Acculturation: Advances in Theory, Measurement, and Applied Research, K. M. Chun, P. B. Organista, G. Marín (dir.), American Psychological Association, 2003, p. 21.

79. Đỗ Khiêm, « Je préfère la langue de la Commune à celle des Versaillais ». 
Il reste que cette acculturation précoce est perçue par les anciens élèves de façon positive. Ils reconnaissent à l'école française un rôle fondamental dans la construction de leur personnalité. C'est grâce à elle qu'ils sont devenus des êtres complets, riches de leur double culture. Ils expliquent leur réussite sociale par leur capacité, acquise via l'enseignement français, à surmonter les épreuves de la vie et à s'adapter à un nouvel environnement social. De ce point de vue, l'école française dans le Sud-Vietnam d'après 1954 est un exemple réussi de transfert culturel. Les milliers de médecins, chercheurs, enseignants, ingénieurs, intellectuels, artistes, diplomates et cadres supérieurs formés dans ces écoles ont été bénéfiques aux pays où ils ont exercé leur profession, y compris au Vietnam pour ceux qui sont restés sur place après $1975 \mathrm{ou}$ pour ceux qui y retournent depuis quelques années. Nous ne devons cependant pas oublier que les témoins consultés pour ce travail sont ceux qui ont survécu aux guerres vietnamiennes et réussi leur vie, grâce à leur talent personnel, mais aussi grâce à leur réseau familial et à la chance. Beaucoup d'autres élèves des écoles françaises, en situation d'échec scolaire, quittèrent le lycée pour aller sur les champs de bataille, et ne sont plus là pour témoigner. 



\title{
11 \\ L'ère des tempêtes
}

\section{Dien Bien Phu, Genève, 1953-1954, sous le regard de quatre périodiques français (Paris Match, L'Aurore, L'Observateur et L'Express)}

\author{
Alain Ruscio
}

1953. Depuis sept années, une partie de l'armée française mène à douze mille kilomètres de la métropole une guerre qui, de plus en plus, paraît sans espoir. En France, l'opinion est ballottée entre l'indifférence et l'exaspération. C'est, selon une formule bien connue de Lucien Bodard, l'enlisement ${ }^{1}$.

Sur le terrain, la lassitude est grande. Après l'espoir d'un redressement spectaculaire, sous le règne du roi Jean ${ }^{2}$, le corps expéditionnaire voit ses positions s'effriter. En mai, le gouvernement René Mayer décide de remplacer le commandant en chef, le général Raoul Salan, par un brillant officier, le général Henri Navarre. Le premier avait bourlingué en Indochine dès les années 1930, il avait été presque continûment sur place depuis le début de la guerre, le second ignorait tout de l'Asie. Justement, rétorquent les partisans de Navarre, c'est un bon point. Un regard neuf s'impose.

\section{Le général Navarre: un nouveau sauveur?}

Un mois plus tard, le nouveau commandant en chef remet au gouvernement un document contenant une analyse de la situation et une série

1. La guerre d'Indochine. L'enlisement, Paris, Gallimard, 1963.

2. Surnom du général (maréchal à titre posthume) de Lattre de Tassigny. 
de propositions, sous forme de calendrier. Moyennant des restrictions quant aux moyens mis à la disposition du corps expéditionnaire, les autorités civiles acceptent ce programme qui va vite devenir, pour la presse et les divers spécialistes, le plan Navarre.

Que disait ce plan?

Dans un premier temps (campagne 1953-1954), il se proposait de contenir l'adversaire, de regrouper les forces du corps expéditionnaire, d'affirmer l'autorité de l'armée de Bao Dai; lors de la campagne de l'année suivante (1954-1955), Navarre pensait pouvoir reprendre l'offensive en partant de ces zones pacifiées, désormais sûres. Alors, la France pourrait envisager de négocier en position de force.

Ce plan est en général fort bien accueilli par la presse française.

Le général Catroux, qui connaissait bien l'Indochine pour en avoir été - il est vrai une courte période - le gouverneur général, écrit dans un article-fleuve du Figaro: « Le plan porte la marque d'un chef qui, refusant de subir la volonté de l'adversaire, est résolu à reconquérir l'initiative ${ }^{3}$. " Un vent d'optimisme semble alors de nouveau souffler sur les sphères dirigeantes françaises. La victoire, que l'on n'espérait plus au printemps 1953, semble redevenue une éventualité plausible à l'été. Le camp français cède à ses vieux démons et multiplie les analyses optimistes. Maurice Dejean, tout nouvellement nommé commissaire général de France à Saigon déclare: la lutte du Vietminh est « sans espoir " parce que "nous sommes les plus forts ${ }^{4}$ ".

\section{L'assaut}

C'est dans ce contexte - militaire, politique, mais aussi psychologique - qu'il faut replacer l'opération Castor, c'est-à-dire le parachutage de troupes dans une vallée du pays thaï au nom peu connu jusqu'alors: Dien Bien Phu, dans la matinée du 20 novembre 1953.

Nul doute, pour la grande majorité des journalistes, que le corps expéditionnaire vient de bousculer l'armée vietminh. Quelque chose de nouveau est décidément en train de se passer depuis l'arrivée du général Navarre. France Soir datée du 22-23 barre toute sa Une, en énormes caractères, de ce titre: "INDOCHINE: RUÉE DE PARACHUTISTES SUR DIEN BIEN PHU. » Puis: " Ce premier succès modifie en notre faveur l'équilibre des forces. " L'Aurore du 23 consacre, elle aussi, l'essentiel de sa première

3. 21 juillet 1953.

4. Saigon, 28 juillet 1953, in L'Année politique, édition 1953. 
page à l'événement: " Nouveau coup de boutoir de Navarre contre les Viêts: des milliers de parachutistes s'emparent de Dien Bien Phu, en pays Thaï, à 290 km d'Hanoi. L'ARMÉE FRANÇAISE CONFIRME EN INDOCHINE LA VALEUR DE SES TROUPES ET L'AUTORITÉ DE SES CHEFS. " Le Figaro du même jour loue "l'attaque surprise". Le Populaire, le quotidien socialiste ajoute, le 25: "La fortune semble sourire, au moins provisoirement, aux troupes franco-vietnamiennes, qui viennent de réussir un sensationnel parachutage en pays thaï. " Les premières descriptions qui parviennent sont propres à emporter l'adhésion. Tout laisse à penser qu'il ne s'agit pas d'une opération sans lendemain: " Il s'agit moins, cette fois, d'un raid de peu de durée que d'une opération à longue échéance » (Le Figaro $^{5}$.

On sait que, durant les premières semaines, il ne se passe pas grand-chose sur le terrain. Les Français renforcent le camp, et Giap n'est pas encore prêt. Cependant, à quelques reprises, au cours du premier trimestre 1954, Dien Bien Phu est évoqué. On retrouve alors les thèmes de toute la guerre d'Indochine dans la presse de cette période. Apparemment, nos armes sont encore maîtresses du terrain et les bandes vietminh en état manifeste d'infériorité. L’un des titres de L’Aurore du 24 février nous apprend que "les Viets battent en retraite de Luang Prabang à Dien Bien Phu ». Le correspondant du Figaro juge, lui, que « les Viêts [...] tardent [...] à se frotter à du dur ${ }^{6}$ ". L'Aurore affirme tout de go: "LES VIETS RENONCENT à l'assaut de Dien Bien Phu . "

D’autres sont plus prudents. Robert Guillain, envoyé spécial du Monde, est l'un des premiers journalistes à se rendre sur place. Il passe un " week-end à Dien Bien Phu " (c'est le titre de son article) à la mi-février. Il avoue y avoir été habité par une vague angoisse devant cette « impression d'être encerclé, encagé, cerné; celle encore d'être vu de partout, que chacun de ses mouvements doit être perçu par l'ennemi, qui plonge ses regards d'en haut, tandis que lui-même, derrière le rideau des forêts, ne nous est visible nulle $\operatorname{part}^{8}$ ".

C'est pourtant dans la presse d'opposition franche à la guerre qu'il faut chercher la première analyse sérieuse de la situation militaire. Le 18 février, un mois donc avant le premier grand assaut, dans L'Observateur, Robert Paret publie un article-synthèse au titre résolument

5. 23 novembre 1953.

6. 8 janvier 1954 .

7. 29 janvier 1954

8. 15 février 1954 . 
à contre-courant: " Le plan Navarre a échoué. " Ce plan avait pour objectif de reprendre l'offensive, de briser la dynamique vietminh. Cinq mois plus tard, il n'en est rien: "Contrairement en effet à ce que voudrait suggérer la propagande officielle, il ne se produit depuis plusieurs semaines, en Indochine, rien qui ne fût très prévisible. Les forces populaires mènent leurs opérations au même rythme exactement que lors des campagnes précédentes ". Au contraire, Navarre est retombé dans le piège de l'édification de camps qui n'arrêtent personne, mais qui entament " la fameuse réserve stratégique que le général Navarre s'était attaché à constituer dans le Delta pour pouvoir, le jour choisi, lâcher quarante bataillons sur les arrières de l'armée populaire ». Et de citer: Muong Sai, Luang Prabang, Seno, Dien Bien Phu, « tous inutiles, incapables de gêner l'armée populaire [...]. Le corps expéditionnaire s’éparpille, s’épuise à la poursuite d'un adversaire insaisissable, se retranche dans des forteresses imprenables, que nul n'attaque, mais dont il lui est interdit de sortir ». Paret concluait durement: " En six mois de nouvelle doctrine, les erreurs de Salan [...] ont été bien plus que reprises, démesurément aggravées ». Au total, la stratégie adoptée était taxée d' "échec [...] le plus lourd depuis le début de la guerre en Indochine ". Quelques mois plus tard, au terme d'une enquête sur la situation militaire en Indochine, L'Express, alors tout nouvel hebdomadaire, conclut: "Les buts que l'on avait fixés à la nouvelle tactique, dite plan Navarre, n'ont pu être atteints ${ }^{9}$ ».

Les Français ont-ils été en mesure de comprendre, par la presse de leur pays, les enjeux réels du combat qui commençait, entre le premier parachutage et le premier assaut? Il est très probable que l'homme de la rue, ne lisant que très irrégulièrement les nouvelles d'Indochine, ne parcourant que les titres des grands quotidiens du soir ou des journaux réputés "modérés » a été plutôt enclin à l’optimisme. L'image d’une armée française, bousculée encore quelques mois auparavant, partie à la reconquête du terrain perdu, reprenant l'offensive sur tous les fronts, portant un rude coup à l'adversaire à Dien Bien Phu, n'a pu que s'imposer inconsciemment à lui.

\section{La première vague}

Aussi est-ce une stupeur sans bornes lorsqu'en deux nuits (13 au 14 mars, puis 14 au 15), le Vietminh enlève, non sans de furieux combats, les points d’appui Gabrielle et Béatrice. Dès le 15 mars au soir, le général

9. 6 février 1954 
Navarre, quant à lui, confie à un de ses amis: " Soyons sans illusions : c'est perdu. Je voudrais bien que les Viets ne recommencent pas ce soir. Il va falloir trouver autre chose ${ }^{10}$. " Le 16, il envoie un télégramme à Paris dans lequel il évoque déjà une possible défaite.

Dien Bien Phu, un peu oublié après les enthousiasmes de novembre, revient en force - et dramatiquement - à la une.

L'inquiétude, l'angoisse ne sont plus loin. Mais, dans un premier temps, on veut croire encore que ces revers ne sont que des accidents de parcours.

D'abord, nous conservons la maîtrise absolue des airs, ce qui nous permet de faire fondre un déluge de feu sur l'ennemi: "Dien Bien Phu: bombardements massifs au napalm par nos avions " titre France Soir en une le 25 mars. Mais, surtout, les pertes des Viets sont tellement importantes qu'ils ne pourront lancer un assaut supplémentaire avant longtemps. L'Aurore du 19 mars affirme que le Vietminh s'est « cassé les dents " sur le hérisson. Robert Guillain, dans Le Monde du 16 mars, n’est pas loin de penser la même chose: "En attaquant Dien Bien Phu, l'adversaire n'a t-il pas commis une faute monumentale?"

Le thème de la contre-attaque sera abondamment utilisé durant toute cette période. Paris Match estime: "Les hommes du colonel de Castries n’ont jamais cessé de contre-attaquer ${ }^{11}$. " Dans France Soir du 30 mars, un énorme titre, en première page, annonce: " FOUDROYANTE CONTREATTAQUE FRANÇAISE À DIEN BIEN PHU. Après des combats acharnés, fantassins et blindés s'emparent d'un point d'appui viet qui tenait l'aérodrome sous son feu."

Ce n'est en rien nier le courage des combattants français que de souligner que de telles affirmations étaient des exagérations manifestes. Il y eut certes des contre-attaques. Mais le corps expéditionnaire resta, le plus souvent, sur la défensive.

Position de principe, a priori, pour remonter le moral d'une métropole de moins en moins enthousiaste? Bluff? Auto-intoxication? Ou incommensurable mépris d'un adversaire qui, par nature, ne pouvait vaincre notre armée? Toujours est-il qu'une partie des observateurs, après les graves revers français de la mi-mars, continue à penser (en tout cas, à écrire et à dire) que le sort des armes nous serait finalement favorable. L'infatigable Lucien Bodard, exactement un mois avant la chute, écrit le 6 avril dans France Soir: " On ne croit plus généralement que Dien Bien Phu puisse tomber. "

10. Cité par Jules Roy, La bataille de Dien Bien Phu, Paris, Julliard, 1963.

11. 10 avril 1954. 
Le maintien d'une telle position eût toutefois été suicidaire. Aussi une partie de la presse française est-elle, dès ce moment, bien moins catégorique. Le spectre d'une défaite majeure se profile. Robert Guillain, dans Le Monde du 30 mars, tempère l'optimisme de certains de ses confrères. Une sortie en force du camp retranché est exclue, affirme-t-il. Comment des soldats non préparés à une telle épreuve, harcelés par un ennemi à la puissance de feu croissante, pourraient-ils franchir de longues distances dans une jungle inconnue?

Il faut pourtant bien reconnaître que c'est dans L'Observateur, presse d'opposition réputée "intello ", que se trouvent les analyses proprement militaires les plus pertinentes. Impression renforcée par l'apparition des articles signés Paul Rossel - clin d'œil évident à l'officier communard et rédigés par Pierre Naville. Le 18 mars, il écrit une phrase assassine, que la réalité pourtant confirmera: "En définitive, c'est le général Giap qui a réussi à fixer l'adversaire dans une position intenable qui, même si elle n'est pas emportée, devra sans doute être évacuée, comme l'a été Na San. » Dans les deux hypothèses, ajoute Naville, ce sera une victoire stratégique pour Giap.

Car l'étau se resserre. À Paris, Saigon ou Hanoi, les responsables civils ou militaires informent les journalistes qu'il n'y a plus guère d'espoir de voir le camp triompher du Vietminh par ses propres forces.

Il faut se résoudre à abandonner progressivement le ton triomphaliste. Peu à peu, le thème de l'héroïsme tend à supplanter les autres.

C'est un souffle dramatique qui inspire une des plus célèbres unes de Paris Match, le ${ }^{\text {er }}$ mai: «Dans l'enfer de Dien Bien Phu, le dernier carré des défenseurs vit sous terre. Des salles d'hôpital ont été aménagées dans les abris pour les blessés. Ce lieutenant, blessé lui-même, qui émerge d'un boyau encadré de sacs de terre, est l'un des héros du général de Castries, dont le monde libre a suivi, bouleversé, pendant un mois et demi, l'épopée dramatique. " La photographie tient d'ailleurs un grand rôle dans l'exaltation de cette épopée. Le 24 mars, L'Aurore publie les premières photos de la bataille (elles ont donc mis dix jours pour parvenir en France). C'est surtout Paris Match qui, sans surprise, va se faire un spécialiste de la publication de photos de la bataille. Du 20 mars au 15 mai, en neuf semaines, l'hebdomadaire consacrera cinq unes à Dien Bien Phu, soit une semaine sur deux, publiera 144 photos du camp retranché. Citons quelques légendes caractéristiques: "Ils écrivent une page de gloire dans le ciel de Dien Bien Phu " (reportage sur l'évacuation des blessés, 3 avril); "Ils ne savaient pas qu'ils posaient pour l'Histoire " ( ( $^{\text {er }}$ mai, photos prises par des soldats eux-mêmes avant le premier assaut); « Ceux 
qui lèvent la main sont volontaires pour sauter ce soir en parachute audessus de l'enfer de la jungle " ( $1^{\mathrm{er}} \mathrm{mai}$, photo représentant des soldats réunis dans une cour de caserne à Hanoi), etc. Il fallait à ces héros une comparaison digne d'eux. Ce ne pouvait être, vu les conditions du combat - rapprochement des assaillants et des défenseurs, violence, fureur des chocs, existence des tranchées - que Verdun. Dès ce moment se constitue la légende de la bataille de Dien Bien Phu, «Verdun de la brousse » (Paris Match, 10 avril), « Verdun de la jungle " (Jules Romains, L'Aurore, 6 mai), «Verdun tropical » (Le Figaro, 15 avril), " Verdun tonkinois " (Progrès de Lyon, 2 avril), etc.

\section{Nos héros face à la ruée jaune}

De ce monde de héros se dégagent, à la lecture de la presse française d'alors, deux figures d'exception: le colonel, puis général, Christian Marie Ferdinand de la Croix de Castries et une jeune infirmière, Geneviève de Galard. Ces deux noms à particule claquent comme un rappel des meilleures traditions chevaleresques de la France éternelle. Le sang bleu contre la vague rouge.

De Castries, d'abord.

Un temps, au printemps 1954, cet officier fut l'homme le plus célèbre de France. Pas de reportage qui ne publie son nom. Pas de fresque sur les héros combattants qui ne le fasse figurer au premier plan. Paris Match consacre sa Une du 3 avril à une photo de de Castries, le chef au combat, le regard lointain sur le champ de bataille. Le même hebdomadaire explique que toutes les conversations entre de Castries et son supérieur direct, Cogny, « se terminent invariablement par ces mots de de Castries: le moral de mes hommes est formidable ${ }^{12}$ ! ». Même après la chute, le héros rassure. Paris Match rapporte cette scène de l'ultime minute du camp: « $\mathrm{M}^{\mathrm{me}}$ de Castries a reçu un dernier coup de téléphone de son mari. Dans le fracas des obus, il lui a dit d'une voix calme: "Ne t'inquiète pas, j'ai déjà été prisonnier. Nous nous retrouverons ${ }^{13}$. "

Puis Geneviève de Galard.

Fait exceptionnel, une femme restera, jusqu'à la fin, dans le camp retranché. La seule femme? La presse l'affirme alors sans vergogne. Elle feint d'ignorer totalement les pauvres filles, envoyées là-bas probablement sans enthousiasme particulier, les prostituées du BMC, qui souvent

12. 17 avril 1954 .

13. 15 mai 1954. 
ensuite se transformèrent en infirmières. Il faut ajouter, hélas, que la mémoire collective ne les a pas non plus saluées. Geneviève de Galard, elle, est immédiatement sous les projecteurs, acquiert en quelques semaines une célébrité immense, figurant en une de Paris Match $^{14}$, de France Soir (six fois en un mois ${ }^{15}$ ), etc. Paris Match évoque "la seule douceur des blessés: le sourire de Geneviève ${ }^{16}$ ". Très vite, elle est surnommée par une grande partie de la presse l'ange de Dien Bien Phu, « un ange de douceur dans l'enfer des hommes ${ }^{17}$ ". Un autre surnom, affectueux, lui est, nous apprend Le Figaro du 6 mai, attribué par les soldats: "Bécassine, à cause de son visage rond, presque celui d’un bébé, [qui] garde, quelles que soient les circonstances, un calme extraordinaire et ne trahit aucune émotion. "

Personne ne songera un instant à mettre en cause le courage effectivement exceptionnel de ces quelques milliers d'hommes largués dans un pays hostile, isolés au centre d'une cuvette dont l'ennemi tenait les bords, privés très tôt de tout contact avec le monde extérieur, vivant, enfin, dans des conditions d’hygiène assez épouvantables, infernales même pour les blessés.

Mais l'exploitation politique et sentimentale de ce courage n'est pas acceptée par l'ensemble de la presse métropolitaine. L'Express du 20 mars, par exemple, refuse aux gouvernants le droit de faire le moindre usage politique, politicien même, de l'héroïsme des combattants de Dien Bien Phu: "L'exemple de leur courage commande, à Paris, de ne plus jamais céder à la démagogie facile - et honteuse - qui recouvre à chaque débat la lâcheté politique derrière le slogan de l'honneur de nos soldats. " Un des correspondants du Monde en Indochine, Charles Favrel, est sévère: "C'est très joli de faire des héros en vrac quand il n’y a pas pour eux d'autres solutions que se faire tuer, mais venez donc un soir faire un tour chez les paras qui doivent sauter dans la nuit, et vous me reparlerez ensuite de leur moral ${ }^{18}$. " Plus polémique, Claude Bourdet, de France Observateur, dénonce ce qu'il appelle la scandaleuse exploitation du drame. Le journaliste-militant ironise sur le courage, vertu française, alors que le camp était défendu, certes par des Français, mais aussi, en grand nombre, par des Vietnamiens (membres du CEF ou de l'armée Bao

\footnotetext{
14. 8 mai 1954.

15. 23, 27 et 28 mai, 2, 3 et 4 juin 1954.

16. $7^{\text {er }}$ mai 1954.

17. Dépêche du Midi, 18 mai 1954.

18. 22 avril 1954.
} 
Dai), des Maghrébins, des Africains, des légionnaires, dont des Allemands, "SS prisonniers [...] libérés sous condition d'engagement à la Légion afin de casser du Viet pour se faire pardonner d'avoir cassé du Français ${ }^{19}$ ». Gilles Martinet signale que la presse de Bonn utilise systématiquement l'expression "soldats franco-allemands ", ce qui, à l'approche du neuvième anniversaire de la capitulation nazie, est quelque peu $\operatorname{amer}^{20} \ldots$

La presse française modérée doit bien, après les premiers succès vietminh, et avant la chute prévisible du camp, abandonner le ton quelque peu condescendant du début de la bataille. Non seulement l'ennemi a accepté le défi que l'on disait mortel pour lui, mais il semble prêt à sortir triomphant du choc.

Deux images complémentaires vont, de mars à mai 1954, s'imposer au lecteur de cette presse: la vague humaine et le fanatisme. Ce qui, chez les soldats du corps expéditionnaire, est à juste titre considéré comme digne de respect, d'admiration même, le courage physique, l'endurance, le mépris du danger, est ici assimilé à quelque chose d'étrange, d'inquiétant. Des hommes, nos adversaires? Non pas. Des êtres hybrides, mi « fourmis verdâtres, casquées de latanier » (Joël Le Tac, Paris Match ${ }^{21}$ ), mi " taupes " (Lucien Bodard, France Soir ${ }^{22}$ ).

L'image que peut avoir la France, à travers cette presse, de son adversaire, est donc celle-ci: des milliers et des milliers soldats, sans peur de la mort, puisque fanatisés, venant sans interruption se briser sur les défenses françaises jusqu'à les percer. À côté d'un anticommunisme toujours virulent ( "Nous avons affaire avec les communistes. Ce ne sont pas des hommes comme les autres ", Robert Lazurick, L'Aurore ${ }^{23}$ ), c'est à ce qu'il faut bien appeler du racisme que de telles descriptions font appel. Les thèmes des masses asiatiques innombrables, de la fourmilière humaine, précédant celui du péril jaune, sont sans cesse sous-jacents. Dès lors, le lecteur de cette presse n'est plus très loin d'imaginer que Dien Bien Phu est une menace directe pour son quartier ou son village. C'est donc plus qu'un combat local: une bataille qui va même au-delà de la simple question de la victoire ou de la défaite dans la guerre d'Indochine. C'est une lutte entre la civilisation européenne - et la barbarie - asiatique: « En ces heures où les Viets communistes se ruent à mort contre les nôtres, écrit, lyrique, Robert Lazurick,

\footnotetext{
19. 13 mai 1954.

20. 8 avril 1954.

21. 15 mai 1954.

22. 23 avril 1954

23. 17 mai 1954
} 
notre pensée va aux drapeaux tricolores qui flottent sur nos postes assiégés, à nos hommes jetés dans une lutte sauvage et qui, corps à corps, défendent, oui, la civilisation, NOTRE CIVILISATION » (L'Aurore ${ }^{24}$ ).

Circonstance aggravante, le Vietminh refuse une trêve. La veille encore de la chute, L'Aurore poursuit sa campagne: « Les Viêts rejettent inhumainement le principe d'une trêve immédiate pour l'évacuation des blessés de Dien Bien Phu! » titre le quotidien le 7 mai. Pire même, l'ennemi n'a jamais voulu respecter les conventions internationales: « Les Viêts tirent sur la Croix-Rouge " (Paris Match ${ }^{25}$ ).

Cependant, cette vague ne porte pas la totalité des organes de presse. Pierre Naville, de France Observateur, juge sévèrement ce qu'il nomme un " chantage humanitaire à des fins militaires ": " Il va de soi que le sort des blessés du corps expéditionnaire à Dien Bien Phu est pitoyable, comme l'est celui de milliers de soldats de l'armée populaire agonisant dans les barbelés du général de Castries ou dans les flammes du napalm. Mais, si pénible que cela soit, à la guerre comme à la guerre. Permettre l'évacuation de centaines d'hommes blessés dans une forteresse encerclée, c'est renforcer du même coup la garnison. Le but des assiégeants, c'est la destruction du pouvoir combatif de l'adversaire. Une garnison affamée et engorgée de blessés et de malades perd beaucoup de son pouvoir combatif. La présence des blessés est un facteur de succès à l'actif des assiégeants. De plus, il faut tenir compte du fait que l'évacuation des blessés ne pourrait se faire que par hélicoptères et avions, sur une piste restaurée, c'est-à-dire par le rétablissement de la ligne de communication et de retraite du corps expéditionnaire; et il va de soi que les avions ne serviraient pas qu'à emporter les blessés ${ }^{26}$. "

\section{Une intervention américaine directe?}

Un autre thème agite les rédactions parisiennes: les États-Unis, notre principal allié, doivent-ils intervenir directement pour sauver le camp? On sait aujourd'hui qu'un plan, baptisé opération Vautour, a effectivement existé. De nombreuses fuites - imprudence ou guerre psychologique? - ont permis aux contemporains d'en avoir, au moins partiellement, connaissance. On sait que cette intervention directe, souhaitée par certains à Paris et à Washington, sera finalement bloquée par le veto du

\footnotetext{
24. 17 mars 1954.

25. 3 avril 1954.

26. 6 mai 1954.
} 
président Eisenhower et par l'opposition de Churchill, qui joua dans cette affaire un grand rôle modérateur.

Pour certains, les choses étaient claires: il fallait que la puissance militaire américaine s'engageât en Indochine. À intérêts communs à défendre, lutte en commun. Dans Paris Match, Raymond Cartier se fait le champion d'un certain jusqu'au-boutisme, souvent critique à l'égard des tergiversations de la diplomatie US. Le 3 avril, il intitule l'éditorial de son hebdomadaire: "Grave décision d'Eisenhower: il jette le poids des USA dans la guerre d'Indochine. " Cartier pose la question: Washington pourra-t-il se contenter d'envoyer en Indochine armes et dollars? Son opinion est faite: la guerre en cours est totalement, quasi uniquement, un des fronts de la lutte gigantesque en cours entre communisme et monde libre. Donc, « L’intérêt de la France est [...] d'attirer l'Amérique le plus loin possible dans une guerre dont le poids actuel ne serait tolérable que pour la défense d'un grand patrimoine national. " Après la chute du camp, ce qui était hypothèse devient exigence. Le choix du patron de Paris Match est fait: la France ne doit pas seulement accepter l'intervention américaine, elle doit la revendiquer. " La lutte armée pour le Sud-Est asiatique n'est plus concevable que si les États-Unis participent à la guerre non seulement avec leurs dollars, mais avec leurs soldats, non seulement avec leur acier, mais avec leur sang ${ }^{27}$. " De Genève, Jean Farran, pour le même journal, se fait l'interprète du ministre français des Affaires étrangères, Georges Bidault, censé s’adresser aux communistes: "Pas plus que vous ne pourrez gagner la guerre d'Indochine, l'Occident n'abandonnera jamais le Vietnam au communisme. S’il le faut, il y enverra des escadres américaines, des porte-avions battant pavillon étoilé, des obus atomiques made in USA. Vous devrez traiter ${ }^{28}$."

La presse française se fait d'ailleurs l'écho de ces divergences jusque dans les milieux dirigeants de Washington. Raymond Cartier critique Eisenhower, encensé quelque temps plus tôt, désormais durement apostrophé après son refus de l'engagement. Cartier dénonce l' énergie déclinante ", la " mollesse », l’" imprécision de pensée » du président, à l'origine d'un " recul dramatique dans la position des États-Unis en face du conflit d'Extrême-Orient ${ }^{29}$ ". Mais la bête noire, en ces semaines, de la presse interventionniste, est la diplomatie britannique, hostile, on l'a vu, à une escalade en Indochine. Winston Churchill est même carrément

\footnotetext{
27. 8 mai 1954.
}

28. Id.

29. 17 avril 1954. 
malmené: le lion superbe "qui n’a pas tremblé devant Hitler » a bien mal vieilli! Mais "le sang n'irrigue plus régulièrement le cerveau du vieillard " écrit, avec une délicatesse infinie, le même Raymond Cartier, dans Paris Match du 8 mai.

En revanche, Cartier ne tarit pas d'éloges sur Richard Nixon, le jeune vice-président, chaud partisan de l'escalade: «Dans l'affaiblissement des caractères provoqué par les contingences des régimes démocratiques, les hommes qui pensent clairement et qui parlent hardiment sont trop rares et trop précieux pour ne pas être salués ${ }^{30}$."

Ce ton extrémiste est cependant relativement rare.

Le 3 avril, L'Express constate: "Il semble aujourd'hui que la guerre d'Indochine risque de devenir bientôt une guerre internationale ». Si on en arrivait à cette extrémité, " un très grand malheur se serait abattu sur l'Occident, et par la faute de notre politique ". Dans le même numéro, François Mitterrand, qui venait d'être élu président de l'UDSR, juge inadmissible une telle éventualité. Plus technique, France Observateur décrit les impossibilités militaires d'une telle intervention. L'article ajoute: les Américains, de toute façon, n'interviendront pas s'ils n’héritent pas du commandement en chef. Le gouvernement français tombera-t-il dans cette ultime lâcheté ${ }^{31}$ ?

\section{La chute}

Finalement, on le sait, le camp tombe le 7 mai.

Pour toute une partie de la presse, le premier réflexe est de saluer les héros - français - de cette lutte titanesque. Les titres barrant toutes les premières pages sont édifiants. L’Aurore titre, le 8 mai: « LA FRANCE FIÈRE DE SES SOLDATS. LA CHUTE DE DIEN BIEN PHU, dont les défenseurs (à bout de munitions) ont été submergés, dramatiquement accueillie dans le monde entier "... Paris Match consacre une Une supplémentaire. Sous la photo du commandant en chef du camp, la légende salue « le calvaire et la gloire du général de Castries et de ceux de Dien Bien $\mathrm{Phu}^{32}$ "... Tous les récits s'accordent alors sur un certain nombre de détails pathétiques: " Héroïques jusqu'au bout, ils sont tombés sans drapeau blanc " (Id.).

Mais se réfugier derrière l'héroïsme est vraiment trop facile. La question des responsabilités ne peut pas ne pas être posée.

\footnotetext{
30. 8 mai 1954.

31. 29 avril 1954

32. 15 mai 1954
} 
Une partie du monde politique et de la presse cède à la formule facile de la trahison de l'arrière, thème qui s'accentuera ensuite, avec les années. La presse d'opposition est saisie, L'Humanité-Dimanche la première (12 avril ${ }^{33}$ ), puis L'Express (29 mai), coupable d'avoir divulgué un rapport très pessimiste des généraux Ély et Salan. Ainsi, ce n’est pas le désastre militaire qui est en cause, c'est l'exposition au grand jour de ce désastre! Le Canard enchaîné, irrévérencieux, montre deux personnages, manifestement policiers en civil, parlant à voix basse. Le premier dit à son compère: "Un certain Thiers et un nommé Michelet répandent le bruit de revers à Crécy et à Waterloo... " (Henri Monier, 2 juin 1954).

Mais cela apparaît par trop comme une diversion. Il faut en venir à l'essentiel. Et on peut compter sur L'Express et L'Observateur pour rappeler les vraies responsabilités.

$\mathrm{Au}$ premier rang du banc des accusés: le Mouvement républicain populaire, parti d'inspiration démocrate-chrétienne, installé au Quai d'Orsay et à divers postes de responsabilités, concernant l'Indochine, presque sans discontinuer depuis 1947. Claude Bourdet pose une question cruelle: "Le MRP est-il le parti du crime? " (titre de l'article). Il commente: « La culpabilité du MRP est si éclatante que les Ramadier, les Pleven, les Queuille, les Pinay, les Laniel s'empresseront de faire oublier les leurs " (France Observateur ${ }^{34}$ ). Le grand écrivain catholique François Mauriac écrit dans son "Bloc-Notes ", que L'Express publie chaque semaine: "Pensée amère, pensée empoisonnée: cette histoire à la fois criminelle et inepte, cette histoire de dix ans écrite à l'encre rouge, l'a été par des chrétiens [...]. Les démocrates chrétiens au pouvoir ont, à mon sens, trahi leur vocation qui était de rendre manifeste cette vérité: la politique n'échappe pas à la loi morale ${ }^{35}$. " Il y revient, un mois plus tard, élargissant sa critique: " Huit ans de massacres qui ont assuré l'équilibre financier de la France, Dien Bien Phu, la répression atroce de Madagascar, la terreur au Maroc et en Tunisie [...], tout cela compte infiniment moins aux yeux des chefs MRP qui en sont responsables pour une part que Dieu seul connaît, tout cela leur importe infiniment moins que l'héritage électoral du RPF et que les perspectives radieuses de: "l'État, c'est encore nous." Oui, l'État, c'est encore vous, c'est toujours vous: l'état dans lequel nous sommes ${ }^{36}$.»

Mais les généraux sont également mis en accusation.

33. "Le cours du sang versé en Indochine monte à la bourse de New York. »

34. 6 mai 1954

35. 8 mai 1954.

36. 12 juin 1954. 
Peu de temps après les premiers revers français à Dien Bien Phu, des voix s'étaient élevées pour dénoncer le choix aberrant de la cuvette. Dès le 2 mai, l'ex-colonel Jules Roy (qui avait servi dans le corps expéditionnaire) devenu journaliste, écrivait dans L'Express: " Dans toute l'histoire militaire moderne, il n'existe sans doute pas d'autre exemple que cette décision de barrer le chemin à l'ennemi en se bouclant soi-même, à $200 \mathrm{~km}$ de toute base de ravitaillement et toutes les routes terrestres coupées, dans un fond de vallée qui n'était qu’une partie de jungle. »

C'est en fait le procès du général Navarre, si encensé un an auparavant, qui commence.

Paris Match, dans des lignes pleines d'émotion, rapporte les derniers propos échangés entre De Castries, à Dien Bien Phu et Cogny, à Hanoi. Significative est cette légende d'une série de photos: "Deux ordres du jour ont marqué la fin tragique de l'héroïque bataille de Dien Bien Phu. Le général Navarre, commandant en chef en Indochine, a déclaré, simplement: "La guerre continue. Le général Cogny, commandant en chef du Tonkin, a rendu cet hommage à ceux qui tombèrent glorieusement sous le coup de l'ennemi: “Compagnons de ces hommes, soyez fiers"37 ". Un général qui veut garder le cerveau froid contre un autre qui a le cœur chaud... L'hebdomadaire ne cache pas vers lequel vont ses préférences.

\section{La fin d'une guerre}

Reste une ultime question, qui n'est pas mineure: que faire à présent? L'insistance mise par la presse pro-guerre sur l'héroïsme de nos combattants est en soi un aveu: plus personne, à Paris Match, à L'Aurore ou au Figaro, n'ose émettre un pronostic favorable quant à l'issue de la guerre. Dans le camp opposé, les choses sont dites crûment: "L'opinion de tous (à l'état-major de Hanoi) est formelle: on ne peut plus tenir le Nord ", tout au plus peut-on espérer contrôler « la route Hanoi-Haiphong en vue d'une évacuation possible du $\operatorname{Nord}^{38}$ ". Un mois plus tard, précisément le 29 mai, c'est L'Express qui porte le coup de grâce aux illusions françaises - si toutefois tel ou tel en entretenait encore — : c'est la publication du rapport Ély-Salan, qui vaudra à l'hebdomadaire une saisie (on sait depuis bien des années que ce fut le général Salan lui-même qui remit ce document explosif à la rédaction de l'hebdomadaire ${ }^{39}$ ): deux des chefs les plus prestigieux

37. 15 mai 1954.

38. 29 avril 1954.

39. Voir Françoise Giroud, «La fondation de L'Express », L'Histoire, n 109, mars 1988. 
de l'armée française, de retour d'Indochine, affirment que la carte de guerre française est " beaucoup plus détériorée " qu'ils ne s’y attendaient, qu'ils craignent, c'est écrit noir sur blanc, " de nouveaux Dien Bien Phu ", que le général Giap est à la veille d’une attaque générale sur le delta, etc.

Or, il faut le rappeler, la partie indochinoise de la conférence de Genève commence précisément le lendemain de la chute du camp. Le grand maître de la diplomatie française d'alors, Georges Bidault, a continûment lié son nom à la politique belliciste depuis 1946. Ses jours sont comptés. Il sera remplacé, on le sait, par Pierre Mendès-France, le 18 juin suivant.

Mais nous ne sommes plus dans le vif du sujet de notre intervention.

\section{Épilogue}

Après Dien Bien Phu vint Genève. On en connaît les clauses, la principale étant la partition, présentée comme provisoire, mais dont chacun sentait qu'elle serait durable, du Vietnam en deux entités.

Une diplomatie française aux abois (sous Bidault), puis tristement réaliste (sous Mendès), une Amérique bien décidée à reprendre le flambeau de la défense du monde libre, et à stopper l'avancée du communisme sur le $17^{\mathrm{e}}$ parallèle, une Union soviétique et une Chine populaire passives, tant elles étaient demandeuses de coexistence pacifique... Que pesaient, face à cette coalition objective qui ne voulait plus entendre parler du Vietnam, l'unité de ce pays? Que pouvaient bien signifier, pour Mendès, Dulles, Molotov et Zhou Enlai, les récriminations convergentes, bien que pour des raisons différentes, de Ho Chi Minh, Pham Van Dong et Ngo Dinh Diem?

Un homme politique avait pourtant été lucide. Dans L'Express daté du 8 mai, vingt-quatre heures après la chute de Dien Bien Phu, il avait écrit: "La solution du partage signifie, pour tous les patriotes vietnamiens, un déchirement insupportable. Elle annonce un irrédentisme, une revendication permanente de l'unité. Elle prépare la lutte contre les puissances tutrices qui, pour leur propre commodité, circonscrivent des zones d'influence. Ainsi, d'un pays qui tend à la cohésion, on fera une marche où voisineront les armes ennemies. Ce pays ne s'y résignera jamais. »

C'était assez bien vu. Il s'appelait François Mitterrand. Dommage qu'il ait oublié ces principes lorsque le gouvernement Pierre Mendès France auquel il participait, en juillet, entérina la division du Vietnam.

Il est vrai que six mois après Dien Bien Phu, un peu plus de trois mois après Genève (les cent jours de l'empire colonial) commençait en Algérie le second conflit de la décolonisation tragique... 



\section{QUATRIÈME PARTIE}

\section{ARTS ET LITTÉRATURE}





\section{Deux mondes artistiques, une rencontre}

\section{Beaux-arts: du contact sous influence à l'émancipation croisée des inspirations Nadine André-Pallois}

L'expansion coloniale au $\mathrm{xIx}^{\mathrm{e}}$ siècle va de pair avec une volonté gouvernementale de diffuser l'image des colonies en métropole, afin de promouvoir et justifier cette expansion. Pour susciter le goût des colonies indochinoises et en faire découvrir toute la richesse, sont publiés les premiers récits d'exploration ${ }^{1}$, les premières photos ${ }^{2}$, sont rapportés les premiers moulages et les premières œuvres d'art, présentés lors des expositions coloniales $(1867,1878)$, puis au sein du nouveau musée indochinois du Trocadéro (1889). Au $\mathrm{xx}^{\mathrm{e}}$ siècle, ces expositions coloniales se veulent désormais proches d'un orientalisme vécu. C'est ainsi que, lors de l'exposition coloniale nationale de Marseille en 1906, sont officiellement invités le roi du Cambodge et les danseuses de la cour dont la grâce et la fluidité des mouvements sauront séduire Auguste Rodin. L’exposition coloniale internationale de Paris-Vincennes en 1931 choisit de reproduire grandeur nature le troisième étage d'Angkor Vat.

Une étape supplémentaire est franchie, au début du xx ${ }^{\mathrm{e}}$, avec le choix de mettre l'art au service de la promotion des colonies, faisant ainsi

1. H. Mouhot, "Voyage dans les royaumes de Siam, de Cambodge et de Laos de 1858 à 1861 », Tour du Monde, 1863 ; Paris, Hachette Bibliothèque rose, 1868. F. Garnier, « Voyage d'exploration en Indo-Chine », Tour du Monde, 1870-1871, Paris, Hachette, 1873.

2. Photographies de Gsell pour l'expédition Doudart de Lagrée 1866-1868, Louis Delaporte 1873 et 1882, Lucien Fournereau 1887; N. André-Pallois, L'Indochine: un lieu d'échange culturel? Les peintres français et indochinois (fin XIXe $-X X^{e}$ siècle), Paris, Presses de l'EFEO, 1997,p. 28 et suiv. 
référence à l'héritage des peintres orientalistes qui avaient su rapporter en France dès la fin du xviII ${ }^{\mathrm{e}}$ siècle les couleurs et la lumière de l'Orient (Afrique du Nord et Proche-Orient). Des bourses de voyage sont ainsi mises en place pour favoriser le voyage des artistes dans les colonies afin qu'ils ramènent de leur voyage des images séduisantes et attrayantes de ces contrées lointaines. Le prix Indochine sera créé en 1910.

Parallèlement, dans le cadre de sa mission civilisatrice, le gouvernement souhaite diffuser l'art français dans la colonie au moyen d'expositions d'artistes français et de la mise en place d'un enseignement artistique. C'est dans ce cadre colonial que naît la peinture vietnamienne moderne, dans le deuxième quart du xx $x^{\mathrm{e}}$ siècle, avec la création, à Hanoi, de l'école des beaux-arts de l'Indochine, produit de la colonisation.

$\mathrm{Au}$ Vietnam, la tradition picturale n'a jamais connu un développement similaire à la tradition picturale chinoise, déjà connue en France. La peinture y a gardé jusqu'au début du xx siècle un caractère artisanal, liée aux estampes populaires et aux décors des pagodes. Par l'intermédiaire des peintres français venus séjourner dans la colonie asiatique, les premières promotions d'élèves vietnamiens s'initient donc à la vision occidentale de l'art.

Dans la perspective du transfert culturel qui est le nôtre, on peut se questionner sur la manière dont ces deux traditions se sont rencontrées et quel a été le fruit de leur rencontre. Quelles images de l'Indochine les artistes français ont-ils retenu dans leurs œuvres? Quel a été l'impact sur eux de la découverte de nouvelles civilisations? Dans l'autre sens, quel fut l'apport de l'enseignement français et comment influa-t-il sur l'œuvre des peintres vietnamiens? Quels transferts s'opérèrent-ils, a priori inégaux puisqu'effectués sous l'égide coloniale?

\section{L'Orient des peintres français}

Les peintres missionnaires qui voyagent en Indochine soit grâce à une bourse de voyage (prix Indochine), soit pour une revue (L'Illustration pour Jean Launois, 1924), soit à l'occasion de la préparation d'une exposition coloniale (François de Marliave, 1922), soit pour y assurer un enseignement (André Maire, 1919 et 1948, Joseph Inguimberty, 1925) sont attirés par la découverte d'un monde nouveau que les différents récits de voyages, photographies, etc. publiés dans les revues telles que Le Tour $d u$ Monde, Le Monde colonial illustré, leur a laissé percevoir, mais dont ils ne savent pas grand-chose. Ils partent au gré des opportunités qui leur sont offertes. 


\section{Premiers regards, un état des lieux}

Les premiers peintres français voyageurs en Indochine à la fin du $\mathrm{xIX}^{\mathrm{e}}$ siècle sont avant tout sensibles à l'exotisme du monde qui s'offre à eux.

Ils s'attachent à représenter des paysages, des architectures où la personne humaine tient finalement peu de place: comme dans Baie d'Along ${ }^{3}$, œuvre de Gaston Roullet qui voyage en Indochine en 1885 comme peintre de la Marine. Seuls font exception les portraits d'empereurs, ainsi que ceux de danseuses cambodgiennes ou d'acteurs annamites, considérés comme des personnages de scène présentant un intérêt historique et non comme des gens de la rue.

C'est ainsi que pendant toute cette première période, sont pris pour sujets les sites les plus représentatifs de la colonie: à Hanoi, le Petit Lac, le quartier chinois, le lac de l'Ouest; la baie d'Halong, le port d'Haiphong; le fleuve Rouge; à Huê, le palais royal et ses cérémonies, la rivière des Parfums, les tombeaux des empereurs Nguyễn; à Tourane, les montagnes de marbre; les plages de Nha Trang et parfois quelques vestiges de tours Cham; les pagodes et les collines boisées de Dalat; le marché de Cholon et les arroyos chinois (Saigon, considérée comme une ville trop européenne est rarement représentée); les bords du Mékong; les pagodes et les Bouddha de Luang Prabang; à Phnom Penh, le Palais Royal; la ville de Siem Reap et les temples d'Angkor Vat et du Bayon...

Hommage est ainsi rendu aux civilisations créatrices de ces architectures civiles et religieuses dont on souligne la richesse et surtout la diversité. Les peintres s'attachent aux lignes des bâtiments, aux détails des motifs décoratifs, autant d'éléments de nouveauté et de différence esthétique. Mais la première place demeure aux rendus naturalistes, centrés sur la description des paysages. Les artistes s'efforcent de restituer les caractères exotiques du pays, c'est-à-dire ce qui d'emblée les a dépaysés, surpris ou séduits. La peinture à l'huile prédomine; les gouaches et les aquarelles ne sont utilisées que pour les esquisses. L'ensemble reste très classique et académique.

\section{Rendus d'humanité}

Le siècle avançant, on constate un changement de comportement des peintres face à l'Indochine, suite à la rupture provoquée par la Première

3. Baie d'Along, huile sur toile, 1885, $100 \times 82$, signé, musée d'Orbigny, La Rochelle, cl. N. AndréPallois. 
Guerre mondiale, à une meilleure connaissance de la colonie et à l'intérêt nouvellement porté à l'ethnologie. Les peintres cherchent à échapper aux stéréotypes et à rendre une image originale de l'Indochine, loin des clichés exotiques.

Les peintres manifestent désormais de l'intérêt pour la population, qu'elle soit cochinchinoise, annamite, tonkinoise, cambodgienne, laotienne ou relevant des minorités ethniques. Ils réalisent des portraits ou mettent en scène leurs personnages dans leurs activités journalières.

Les motifs de la vie quotidienne, d'apparition anecdotique durant la période antérieure, tendent à s'imposer: c'est un débordement de chars à bœufs, de buffles, d'éléphants, dans une végétation exubérante, sous les cocotiers. Les personnages sont saisis dans leur univers quotidien: la rizière, les maisons sur pilotis ou à étages, les sampans, les processions religieuses, les marchés. Ce sont des paysans, des pêcheurs ${ }^{4}$, des marchands, des jeunes femmes avec leurs enfants, des religieux ${ }^{5}$. Les peintres aiment représenter tout le particularisme des minorités ethniques en mettant en valeur leurs costumes et leurs attitudes. Ils cherchent à appréhender ainsi leurs différences.

On décrit avant tout les activités des gens modestes: les paysans en train de repiquer le riz, le pêcheur avec son filet à balancier, les marchands ambulants ou ceux possédant un petit commerce, les artisans, les acteurs. Les artistes ne peignent que très rarement les lettrés, les dignitaires, hormis dans des portraits officiels. Cela donne en réalité une image très populaire de l'Indochine. En revanche, les peintres décrivent bien la vie religieuse: moines faisant l'aumône, effigies des divinités (qui n'offrent plus cet aspect monstrueux qu'on leur prêtait avant la conquête), scènes révélatrices des croyances populaires, temples et architectures diverses.

La majeure partie des scènes se déroulent en extérieur: dans la rue, dans les champs, sur les bords d'un fleuve ou d'une rivière, autour de la maison... C'est avant tout là que la vie se déroule. Mais il faut bien dire aussi que figurer des scènes d’intérieur eût impliqué que le peintre y eût été introduit. Or, les rapports des artistes coloniaux avec la population locale restèrent toujours réduits...

4. Par exemple, André Maire, Le Port de Saigon, fusain et sanguine, 1952, coll. part., cl. Mme Harscoët-Maire.

5. Par exemple, Evariste Jonchère, Quête des bonzes avec femmes au bain, huile sur toile $100 \mathrm{x}$ 60, non signé non daté, conservatoire d'Art et d'Histoire de haute Savoie, Annecy, cl. L. Fontani. 
La femme est l'un des sujets favoris des peintres: ils apprécient particulièrement la femme vietnamienne ${ }^{6}$, même si ses dents teintées de noir entraînent souvent un mouvement de recul. La ligne ample et floue de son aó dài et de ses longs cheveux charme les artistes. Mais ils ne manquent pas d'intérêt non plus pour les Laotiennes, les Cambodgiennes ou les femmes des minorités ethniques avec leurs vêtements colorés, leurs bijoux si particuliers... Cette fascination picturale pour la femme indochinoise a son pendant, nul ne l'ignore, dans la littérature coloniale.

À compter des années 1920, certains, comme Jean Launois (18981942), affirment dans leur correspondance avoir souhaité prendre du recul dans l'exploitation de tous ces motifs, et avoir eu conscience de la nécessité de s'habituer à leur environnement, avant de se mettre à peindre: "Il ne faut pas de pittoresque. Il faut le fuir et c'est dans le vulgaire que se trouve la vérité et c'est la seule chose que l'on ne voit pas $^{7}$. " Il faut, dans la mesure du possible, laisser derrière soi son passé, comme le déclare de son côté Henri Dabadie (1867-1949): "J'ai à vaincre le problème d'une autre atmosphère, d'une autre lumière. Je dois oublier et m'employer à dégager la beauté particulière de ces pays ${ }^{8}$. » Ces peintres témoignent ainsi du désir d'aller au-delà de cette première impression de surprise et d'enthousiasme due à la découverte d'un monde nouveau. Ils souhaitent se départir de leurs yeux d'Européens qui les rendent si réceptifs aux nouveautés qu'offrent ces pays. Chacun d'eux réagit ensuite selon la durée de son séjour, ses précédents voyages, sa sensibilité.

Parallèlement, on constate une utilisation nouvelle de la gouache et de l'aquarelle, Cette évolution correspond, dans le temps, à un changement dans le choix des thèmes. Les gouaches et les aquarelles ont d'abord été utilisées (voir supra) pour réaliser les esquisses. Après la Première Guerre mondiale, elles sont considérées comme des œuvres finies et non plus seulement comme des travaux préparatoires. Ces techniques à l'eau, plus faciles d'emploi dans ces pays chauds et humides, offrent la possibilité d'un travail spontané. L'artiste peint sur le motif sans souci de temps de séchage; il rend instantanément la scène qui se présente à lui, un instant fugitif, ce que l'huile ne peut lui permettre.

\footnotetext{
6. Par exemple Joseph Inguimberty, Femmes devant un étang de lotus, huile sur toile Rep. dans

P. Gourou, «Les Beaux-Arts en Indochine », France-Illustration, n² 190, 1949.

7. Correspondance, Hanoi, 22 avril 1924, voir Jean Launois p. 141.

8. P.A. Foinet, « L'Art en Cochinchine. Les peintres: Mme Martin-Gourdault, M. Henry Dabadie », Extrême-Asie, juillet 1929, p. 590.
} 


\section{Le périple de Jean Bouchaud ${ }^{9}$}

Les archives de ce peintre voyageur (1891-1977), autodidacte, prix Indochine en 1924, ont été conservées et mises en valeur par sa famille, qui a reconstitué son itinéraire d'après sa correspondance, ses Relations de voyages et ses carnets de croquis $^{10}$; un itinéraire qui diffère peu de celui qu'ont généralement suivi les peintres à travers les cinq pays de l'Indochine et la Chine du $\operatorname{sud}^{11}$.

Septembre-décembre 1924: Jean Bouchaud embarque à Marseille le 26 septembre 1924 et arrive à Saigon, après presque trois semaines de navigation. Là, il visite probablement la ville européenne, la rue Catinat et ses cafés, le palais du gouverneur, le jardin public et Cholon avec ses maisons à étages et ses pagodes aux toits recourbés. Quittant Saigon, il passe un peu de temps dans le delta du Mékong, puis rejoint Mytho et remonte le Mékong jusqu'à Phnom Penh. Là, le peintre profite de la ville et du spectacle des danseuses de la cour royale. Phnom Penh n'est en réalité qu'un point de passage vers Siem Reap et les temples d'Angkor qu'on rejoint par bateau jusqu'au Tonle Sap, puis par charrette à bœufs. Laissant le Cambodge, Jean Bouchaud rebrousse chemin vers Saigon, afin de repartir vers Dalat, la ville au climat tempéré. Il traverse les régions montagneuses pour rencontrer les populations montagnardes. De là, il rejoint Saigon et embarque pour Haiphong, avec une escale à Tourane (Danang). On ne sait pas trop s'il visite les vestiges Cham ou les montagnes de marbre. À Haiphong, il reprend le bateau pour visiter la baie de Ha Long, puis il gagne Hanoi en train. La capitale tonkinoise est célèbre pour son quartier européen, son opéra dans le style de celui de Paris, son quartier chinois aux rues spécialisées (rue des Tasses, de la Soie, etc.), pour le Petit lac, le lac de l'Ouest, la pagode du Corbeau... Bouchaud s'aventure ensuite dans les hautes régions du Tonkin, en train et en camion, en direction de Lang Son, Cao Bang ou Lao Cay.

Janvier-juin 1925: Retour à Hanoi; Bouchaud se dirige en chemin de fer vers Vinh puis en voiture vers Nappe, et enfin Thakhek où il prend la chaloupe fluviale pour Vientiane; de là, expédition à pied et à cheval pour atteindre Luang Prabang. Bouchaud séjourne deux mois au Laos, qui l'enchante. Le pays particulièrement Luang Prabang - est en général très apprécié des peintres, pour son art et ses architectures bouddhiques, pour la sérénité qui règne dans la ville, pour sa population. Retour à Vientiane, dont les séductions sont moindres, par le Mékong, en radeau, puis Bouchaud se rend à Savannakhet en

9. L'auteur de cet article remercie la famille Bouchaud de son continuel soutien dans ses travaux de recherche.

10. « Un peintre en Indochine 1924-1925 », Carnets du Vietnam, hors série 2010, 59 p.

11. La France obtient par le traité du 10 avril 1898, la concession d'un chemin de fer du Tonkin à Yunnanfou - Kunming. 
chaloupe fluviale. Ile de Không. Rejoint Huê en voiture via Quang Tri. Arrivés à Huê, capitale des empereurs Nguyễn, les artistes s'arrêtent généralement quelque temps, espérant être reçus par l'empereur et visiter le palais impérial. Ils admirent les tombes de ses ancêtres Nguyễn et profitent de la rivière des Parfums. C'est probablement ce que fit le peintre. Retour à Hanoi; Bouchaud prend le train jusqu'à Kunming, capitale du Yunnan, où il se sent complètement dépaysé. Retour à Haiphong en train puis par bateau jusqu’à Saigon. Il embarque à Saigon le 18 mai 1925 après sept mois passés en Indochine.

La correspondance de Bouchaud est riche d'enseignement. Il se plaint du climat " très malsain » et de ne pas être toujours en état de travailler, faute de santé ou de moral: « quand on s'arrête seulement cinq minutes pour faire un croquis, on commence à éprouver un petit malaise " (En route pour Siem Reap, 31 octobre 1924). Il souffre aussi parfois de solitude, isolé dans un monde qu'il ne comprend pas toujours et où il n'est pas compris. Il note son regret de ne pas parler la langue des diverses populations qu'il rencontre, pour leur expliquer ses motivations et pour que lui-même comprenne un peu mieux leur mode de vie.

Bouchaud préfère de loin les régions reculées. L’administration française, qui fonctionne dans les villes, y est absente: "La brousse est inexplorée et insoumise. Pas de route pour y aller: danger. La carte laisse cela en blanc " (Dalat, 17 novembre 1924). Il est conquis par le Laos ("paradis terrestre dont tout le monde me parlait mais [je] n’espérais pas pouvoir y pénétrer; le voyage était long, difficile, pas de routes, peu d'Européens y habitaient ") où il réalise de nombreuses œuvres ${ }^{12}$ qui illustrent ses notes: $A u$ bord $d u$ Mékong, Laos (croquis): «On est pris par cette population simple et dont la vie heureuse et paisible est si loin de notre vie fiévreuse et trépidante "; Le pont en zigzag, Luang Prabang (aquarelle): « Ici les gens vivent heureux avec des fleurs dans les cheveux. Ils ne travaillent pas et vivent tout de même dans le bien-être. C'est Tahiti de jadis "; Femmes dans le torrent, ile de Khong, Laos (huile sur carton): "Ici c'est la paix, la confiance, la joie de vivre ». Au-delà du Laos, il note que "Yunnan Fou est très pittoresque, très très chinois ": Femmes Lolo, Yunnan Fou, 1925 (aquarelle et gouache).

Jean Bouchaud ramène ainsi de son voyage de nombreux croquis, au dessin vif, destinés à noter des attitudes ou des gestes de la vie quotidienne, qu'il réutilisera dans des huiles sur toile au rendu plus formel. Dans Le Chasseur, Dalat 1924 (dessin), il libère, par un trait léger, le mouvement d'un chasseur saisi au repos, appuyé à un tronc d'arbre. On décèle un souci ethnographique dans la précision avec laquelle il soigne un visage ou une attitude particulière, comme dans Le Joueur de musique, Laos 1925 (aquarelle).

Ses aquarelles, ses gouaches et ses quelques huiles tentent de rendre cette luminosité si particulière à l'Indochine: une lumière qu'il juge voilée par

12. Toutes les œuvres citées sont conservées dans des collections particulières. 
l'humidité, une lumière qui n'est pas semblable à celle de l'Afrique du Nord, plus franche et plus éclatante. Il la rend au moyen de couleurs chaudes, relativement sombres (verts, ocres), appliquées sur le support avec une touche légère, égayées de quelques taches blanches, jaunes, roses ou rouges. Sous ses couleurs, on note la présence d'un tracé qui donne une précision à la composition, comme dans La rivière des parfums (gouache), avec en commentaire: "Mer d'un rose sale, montagne ravissante. ». Un paysage est rarement représenté pour lui-même. Il constitue toujours la toile de fond d'une scène de genre.

\section{L'école des beaux-arts de l'Indochine}

Une nouvelle étape dans leur perception de l'Indochine est encore franchie par les peintres français avec la création de l'école des beauxarts de l'Indochine (ÉBAI), instituée le 27 octobre 1924, par un arrêté du gouverneur général Merlin,

La notion d'école des beaux-arts n'a pas d'antécédent en Indochine. C'est une création d'esprit purement occidental. On a l'idée d'une école qui tenterait de concilier les traditions locales avec une approche occidentale de l'art, de faire vivre ou revivre ces traditions grâce à des moyens nouveaux.

L'ÉBAI ne sera donc pas la simple transposition des écoles des beauxarts de métropole. Il s'agit de façon intentionnelle de tenir compte de l'histoire des civilisations indochinoises et de réaliser une synthèse entre les arts de l'Occident et de l'Extrême-Orient.

L'école peut s'appuyer sur le modèle des écoles d'arts appliqués de création antérieure, telles celles de Thu Dau Mot (fondée en 1901: ébénisterie, sculpture sur bois, incrustation, laque); de Bien Hoa (1903, bronze et céramique); de Gia Dinh (1913, enseignement du dessin, de la gravure sur bois et sur cuivre et de la lithographie); de Phnom Penh (1917, fondation de l'école des arts cambodgiens, pour l'enseignement de l'art traditionnel). Ces établissements sont nés d'une volonté du gouvernement colonial de valoriser le travail des artisans, de mettre en contact ces traditions avec le monde moderne, afin d'assurer la vente des produits et donc la survie des artisans. Le but de cette nouvelle école des beaux-arts est cependant de former des artistes, et non plus des artisans, ainsi que des professeurs de dessin pour l'enseignement franco-indochinois ${ }^{13}$.

13. Centre des Archives nationales du Vietnam, Hanoi RST H.4094/73413 (R. 16) : lettre du chef du service de l'enseignement public du Tonkin à Monsieur le Résident supérieur au Tonkin, 26 janvier 1917. Lettre de M. Plantie, administrateur des Services civils à la retraite à Monsieur le Résident supérieur au Tonkin, 23 février 1917. Voir aussi bibliothèque de I'INHA, Paris, Fonds Victor Tardieu, archives 125, 05, 01, création de l'école. 
Son directeur est choisi parmi les anciens titulaires du prix Indochine ${ }^{14}$. Deux directeurs se succèdent jusqu'en mars 1945 (date du coup de force des Japonais qui entraîne une fermeture temporaire de l'école): Victor Tardieu (1925-1937) et Évariste Jonchère (1937-1945). Les enseignants sont des peintres français également titulaires du prix Indochine, mais aussi d'anciens élèves de l'école. Les futurs artistes sont initiés aux techniques occidentales (peinture à l'huile, lois de la perspective...) et extrême-orientales (peinture sur soie, laque...). De même, les modèles sont occidentaux (imitation de la nature), mais aussi traditionnels (chinois et annamites).

Les peintres-enseignants français se révèlent très divers de par leur personnalité, leur itinéraire et leurs travaux, mais présentent un trait commun: tous ont suivi une formation académique et aucun ne témoigne d'un caractère très avant-gardiste. Ils se distinguent par leur attrait marqué pour la personne humaine, des rendus personnels, avec une influence sensible des courants de la fin du xix ${ }^{\mathrm{e}}$ siècle, et le souci d'aller au-delà de l'exotisme qu'offre cette région du monde. Il serait malaisé de réunir ces peintres dans un mouvement particulier. Chacun présente un parcours différent: leurs correspondances se font plutôt l'écho de mésententes. Situation d'autant plus explicable que n'émerge aucune personnalité phare, autour de laquelle les uns et les autres se seraient retrouvés, et que fait défaut une structure d'accueil et de séjour pour artistes non enseignants: l'ÉBAI n'est pas la Villa Abd El Tif à Alger. Il faut noter enfin que les peintres voyagent seuls à travers l'Indochine, ils sont isolés et souffrent de cet isolement.

Cependant, si cette école n'est pas un lieu de rencontre entre peintres français (mais cela n'était précisément pas sa raison d'être), c'est par contre une opportunité pour les professeurs-artistes français de côtoyer les futurs artistes vietnamiens, ce que peu de peintres-voyageurs français n’avaient réussi à faire jusqu'à présent. De là naîtront des convergences mais aussi des divergences de points de vue, des adhésions ou des rejets.

Parmi les figures marquantes ${ }^{15}$ de l'ÉBAI, tout d'abord, Victor Tardieu ${ }^{16}$. Prix Indochine 1920, dans les faits à l'initiative du projet

14. Le prix Indochine, mis en place en 1910, comporte un subside en plus de la gratuité du voyage aller-retour. Ce prix est remodelé en 1926, suite à la création de l'EBAI. Le boursier doit désormais partir deux années consécutives. La première année est consacrée au voyage d'étude dans la colonie. II est ensuite astreint à une année d'enseignement à l'EBAI. Une exposition de ses œuvres est organisée à Hanoi ou Saigon avant son départ.

15. Du Fleuve Rouge au Mékong. Visions du Viêt Nam, Paris musées, éditions Findakly, 2012.

16. Les archives familiales de Victor Tardieu sont conservées à la bibliothèque de l'INHA, Paris. 
d'école et le premier directeur de l'établissement comme rappelé ci-dessus, il honore notamment, en 1921, une commande destinée au grand amphithéâtre de l'Université indochinoise ${ }^{17}$ : c'est la représentation d'une femme symbolisant la métropole entourée d'un côté par les mandarins de la cour de Hué, de l'autre côté par les populations indochinoises... C'est un peintre académique, très respecté par ses élèves.

Il convient ensuite de citer le vietnamien Nam Sơn. Il assiste Victor Tardieu dans sa tâche avant de prendre la responsabilité des cours d'arts décoratifs. Il est important de souligner son rôle, rare à l'époque pour un Indochinois. Nam Sơn, issu d'une famille de lettrés, reçoit une instruction à la fois vietnamienne (il est initié à la calligraphie et à la peinture sur soie) et occidentale. Il entre dans les services civils de l'administration coloniale. Puis il séjourne à Paris où il poursuit un enseignement artistique à l'école des beaux-arts, avant de revenir à Hanoi assister Victor Tardieu. Premier enseignant vietnamien de l'ÉBAI, il enseigne dans le premier cycle, jusque vers 1945; il contribue ainsi à l'introduction d'une instruction, sur le plan des arts décoratifs, spécifiquement indochinoise. On lui doit par ailleurs des portraits réalistes, pleins d'expression.

Évariste Jonchère, peintre et sculpteur, prix Indochine 1932 et second directeur de l'école à la mort de Victor Tardieu, se signale par son intérêt marqué pour les ethnies minoritaires et leur costume traditionnel.

Joseph Inguimberty, professeur contractuel — il n'est pas prix Indochine, enseigne les arts décoratifs en deuxième cycle de 1925 à 1945. Il organise dans les années 1930, avec l'accord de Victor Tardieu, un atelier de laque, afin de remettre en valeur cette technique traditionnelle en Indochine. Linitiative connaît un large succès. Ce peintre apprécie les huiles sur toile de grande dimension, réalisées à partir d'esquisses préparatoires. Ses thèmes sont les paysages du delta, les rizières où s'élève un dinh (maison communale) ou un temple, sous des cieux chargés; la femme tonkinoise, représentée seule, avec son enfant, ou en groupe. Sa peinture témoigne d'un style caractéristique: perspective linéaire, simplification des formes, couleurs cernées posées en à-plat au pinceau ou au couteau, tonalités dominantes ocre et vertes.

Enfin Alix Aymée qui fut l'élève de Maurice Denis, séjourne à différentes reprises en Indochine grâce à son mari, puis, séparée de ce dernier, à la faveur d'une mission qui lui est donnée par le gouvernement général de l'Indochine pour la préparation de l'exposition coloniale de 1931. Ayant beaucoup circulé en Indochine, notamment au Laos et fait œuvre de peintre, elle devient professeur de laque à l'ÉBAI en 1934. Rares sont

17. Bibliothèque de I'INHA, fonds Victor Tardieu. 
alors les peintres français qui se sont initiés à la technique de la laque, très difficile et demandant un long apprentissage. Elle participe notablement à son regain.

\section{La peinture à la laque}

Elle a été développée par les Chinois et les Japonais qui, comme les Vietnamiens jusque dans les années 1930, ne l'ont utilisée que pour la réalisation d'objets décoratifs. Les artistes vietnamiens vont aller au-delà de ce caractère décoratif et explorer toutes ses possibilités. J. Inguimberty est sans doute à l'origine de ce développement, grâce à sa décision d'appeler auprès de lui à l'école des beaux-arts de l'Indochine des artisans laqueurs pour instaurer un enseignement de cette technique. Cela nécessitait tout d'abord de retrouver précisément les étapes de création d'un panneau à la laque, afin d'éviter les problèmes de séchage, de craquelures et d'oxydation des couleurs. Les recherches continuent alors dans de meilleures conditions. Nguyễn Gia Trí est l'un des principaux acteurs vietnamiens de ce renouveau. Il contribue, par ses recherches, à enrichir la palette de couleurs des panneaux (trouver des pigments en accord avec cette matière), alors que le ponçage et le polissage permettent de multiplier les nuances, de les fondre les unes dans les autres, de donner du relief à l'ensemble

De leur côté, les élèves vietnamiens s'initient à la vision occidentale de l'art: imitation de la nature, perspective linéaire, peinture en plein air, dessin d'après modèles vivants. Les techniques propres aux mouvements impressionnistes et post-impressionnistes du $\mathrm{XIX}^{\mathrm{e}}$ siècle qu'ils adoptent n'entrent pas en opposition avec leurs traditions, mais au contraire les renouvellent; sur ce plan, l'école satisfait à ce qui fut le but de sa création. Ils se spécialisent selon les cas dans les techniques de l'huile, de la soie ou de la laque et ont en commun de chercher très vite à s'affranchir des perspectives académiques qui leur sont proposées. La laque, par exemple, est appelée à devenir un moyen d'expression artistique spécifiquement vietnamien. Les peintres vont aller au-delà de son caractère décoratif et en explorer toutes les possibilités. Leur rendu peut être qualifié de tradition dans la modernité: la peinture est de style figuratif; pour rendre la perspective, ils adoptent une composition synthétique qui se rapprochent le plus des plans superposés des peintures traditionnelles des lettrées chinois; les à-plats de couleurs cernés ou modulés sont ce qui leur semble le plus proche de la variété de nuances et d'intensités des lavis anciens.

Parmi les nombreux peintres issus de cette école, on peut citer: Lê Phổ (promotion 1925-1930). Il adopte dans ses œuvres la composition 
synthétique qu'il allie à des couleurs sobres qui s'accordent bien avec la technique traditionnelle de la soie: L'âge heureux ${ }^{18}$.

Nguyễn Phan Chánh (promotion 1925-1930) préserve dans son œuvre sur soie la simplicité des effets, l'économie des traits et des couleurs, à la manière des peintres lettrés chinois. Il apprécie les scènes intimistes, aime jouer avec les contrastes d'ombre et de lumière et avec la trame de la soie: Expérience médiumique ${ }^{19}$.

Tô Ngọc Vân (promotion 1926-1931) privilégie la beauté de la femme des villes qu'il rend avec des à-plats de couleurs de la composition synthétique: Deux jeunes filles avec un bambin ${ }^{20}$. Il sera le directeur de l'école de 1950 à 1954 .

Le nom de Nguyễn Gia Trí (promotion 1931-1936) reste lié à la remise en valeur de la technique de la laque, (cf. encadré ci-dessus). Il apprécie particulièrement les grands formats ou de longues silhouettes féminines évoluent dans des jardins fleuris. Peu soucieux de la réalité, le peintre se laisse plutôt aller à la rêverie qu'il suscite grâce à une technique d'une grande finesse. Malgré les contraintes inhérentes à la laque, les lignes sont fluides, les tissus volent, les figures semblent en mouvement. Le peintre referme l'espace du tableau sur une succession de plans colorés: Jeunes femmes dans un jardin ${ }^{21}$.

\section{Les fruits de la rencontre}

Les artistes vietnamiens vont aller au-delà des acquis et ajouter leur propre sensibilité. La rupture de la guerre va permettre à ces artistes de prendre du recul par rapport à ce qui leur a été inculqué: guerre de résistance contre les Français (1945-1954), accession à l'indépendance, partition du pays en deux, guerre pour la réunification menée contre les États-Unis (1954-1975). Durant la première guerre d'Indochine, de nombreux artistes vont gagner le maquis au Nord du Vietnam, dans le Việt Bắc et redécouvrir l'art traditionnel national, mis fortement en valeur dans un pays qui lutte pour son indépendance. L’aspect traditionnel des villages vietnamiens, peu marqués de l'empreinte française, les

18. L'âge heureux, 1930, peinture sur soie, 127 x 178, coll. part. Cl. N. André-Pallois.

19. Expérience médiumique, 1931, 63 × 87, coll. part. Rep dans Les peintres de l'École supérieure des beaux-arts de I'Indochine, Hanoi, 1993, nº 10.

20. Deux jeunes filles avec un bambin, huile sur toile, 1944, $102 \times 77$, musée des Beaux-Arts du Vietnam, Hanoi. Cl. N. André-Pallois.

21. Jeunes femmes dans un jardin, laque, 1939, musée des Beaux-Arts du Vietnam, Hanoi. Cl. N. André-Pallois. 
sculptures des pagodes, des maisons communales, autant de thèmes qu'ils vont reprendre dans leurs tableaux, donnant corps ainsi à la tradition renouvelée.

Nguyễn Tư Nghiêm (promotion 1941-1945) offre avec Danse ancienne $e^{22}$ une composition liée à un thème populaire au Vietnam et en Asie du SudEst en général, qui dans le même temps fait référence aux avant-gardes occidentales du début du $\mathrm{xx}^{\mathrm{e}}$ siècle, à travers une reconstruction de l'espace à la manière cubiste, retenant plusieurs points de vue d'une même figure. Il exploite une perception géométrique sur le thème traditionnel des danses populaires et réussit ainsi à faire d'un thème familier un motif nouveau, en renouvelant les formes. De même qu'en France, Picasso développa ses recherches formelles à partir de l'exemple des sculptures africaines, Nguyễn Tư Nghiêm, lui, s'en réfère à la tradition vietnamienne qu'il met ainsi en valeur.

Bùi Xuân Phái (promotion 1941-1945) est resté longtemps le peintre vietnamien le plus connu à l'étranger, du fait sans doute de la censure dont il fut l'objet dans son pays jusqu'en 1984. Dans ses œuvres, cet artiste dépeint à la manière d'une esquisse rapidement brossée le caractère pittoresque de la vie quotidienne dans une rue de Hanoi, ou bien les personnages du théâtre populaire. L'espace est construit à partir d'un point de vue unique et mis en mouvement au moyen d'un large cerne qui enserre les volumes irréguliers dont les couleurs sont posées en à-plats modulés: Jour de pluie ${ }^{23}$. On reconnaît à la fois le trait accentué des peintures populaires et les emprunts au monde occidental (à Rouault notamment). Son travail se caractérise par une sincérité d'expression, une richesse des couleurs et de la matière, et enfin un graphisme puissant.

Nguyễn Sáng (promotion 1940-1945) travaille beaucoup les portraits de femmes: Jeune fille aux lotus ${ }^{24}$. L'ensemble est très puissant et les figures hiératiques, alors qu'une note moderne se dégage de l'agencement de ses volumes et du travail de la couleur.

Si les artistes vietnamiens lièrent très vite les deux traditions, il n'en alla pas de même du côté des artistes français. Ce n'est que très progressivement que se fit jour un certain recours aux techniques orientales, grâce à l'ÉBAI qui catalysa les recherches et qui apporta une meilleure connaissance des techniques picturales traditionnelles. Mais au-delà de

22. Danse ancienne, gouache sur papier, 1965, $45 \times 64$. Rep dans Uncorked Soul, contemporary Art from Vietnam, Hong Kong, Plum Blossoms, 1991, p. 81.

23. Jour de pluie, huile sur toile, 1972, $50 \times 67$, coll. part.

24. Jeune fille aux lotus, huile sur toile, 1972, 95 x 130, coll. part. 
cette méconnaissance, ce sont surtout les préjugés culturels dus à la colonisation qui retardèrent leur utilisation par les artistes français. Ceux qui s'y adonnent sont relativement nombreux aujourd'hui. Pour s'en tenir à l'art de la laque: au-delà de la création au sein de l'ÉBAI, de l'atelier dans lequel s’impliqua Alix Aymé, il convient de mentionner l'itinéraire et l'œuvre de Saint-Maur ( $c f$. encadré).

\section{Samuel Saint-Maur (1906-1979) ${ }^{25}$}

Ce fils d'un médecin de marine, après avoir interrompu son propre engagement dans la marine marchande pour obéir à sa vocation artistique, développe un parcours atypique; fondateur en 1935 de l'association de l'art mural, engagé en Espagne en 1936, rattrapé à Pondichéry par la déclaration de guerre et mobilisé, envoyé en Indochine, démobilisé en 1941 mais ne pouvant rentrer en France, il s'installe à Yen Phu, petit village près de Hanoi, sur la digue du fleuve Rouge. Là, il découvre et exploite de nouveaux supports, tel que le papier d'Annam et de nouvelles techniques, celle de la laque. Il organise un atelier de laques où il produit des panneaux laqués, meubles et paravents ainsi que des objets décoratifs. Après le coup de force japonais de mars 1945, il rejoint le Yunnan puis Calcutta avant de regagner la France.

Les œuvres de son atelier sont exposées à plusieurs reprises à Hanoi. En France même, une exposition de ses œuvres sur laque a lieu en 1948 au musée Cernuschi.

Peu d'artistes français se sont aventurés à travailler la laque: la technique, de tradition indigène, est particulièrement difficile. Saint Maur confirme en s’y adonnant avec originalité et succès son caractère d'artiste à bien des égards exceptionnel. La dimension militante, révolutionnaire (se détacher de ses contemporains, rejoindre la cause vietnamienne) n'est pas absente de l'aventure. Reste à étudier précisément quels furent les rapports de SaintMaur avec les artistes laqueurs vietnamiens.

L'esprit d'ouverture et la fécondité transculturelle de cet artiste se traduit également par l'influence qu'exercent sur lui les formes nouvelles de la peinture chinoise. Il prolongera ses recherches visant à allier l'idéogramme chinois à l'abstraction occidentale par l'élaboration d'une "sculpture linéaire suggérant le mouvement et dite écriture d'espace " (Petit Journal, musée de Compiègne, 1980). Il joue également dans ses sculptures avec la dialectique du plein et du vide de la peinture chinoise.

25. Samuel de Saint-Maur, entretiens avec la famille, années 2000. 


\section{Bibliographie:}

Du fleuve rouge au Mékong, visions vietnamiennes, Paris musées, musée Cernuschi, éditions Findakly, 2012, 120 p., ouvrage collectif.

Essays on Modern and Contemporary Vietnamese Art, Singapore, Singapore Art Museum, 2009, 398 p., traduit en vietnamien, ouvrage collectif.

Post Doi Moi. Vietnamese Art After 1990. Singapore, Singapore Art Museum, 2008.196 p., ouvrage collectif.

« Un peintre en Indochine 1924-1925 », Carnets du Vietnam, hors série 2010, 59 p., ouvrage collectif.

N. André-Pallois, "Le renouveau de la peinture à la laque au Vietnam: l'impact de la colonisation. ", La Question de l'Art en Asie Orientale, F. Blanchon (dir.), Paris PUPS, 2008, p. 345-356.

N. André-Pallois, « L'école des beaux-arts de l'Indochine: une création d'esprit occidental ", Banquier, Savant, Artiste, Présences françaises en ExtrêmeOrient au $x^{e}$ siècle, F. Blanchon (dir.), Paris, PUPS, 2005, p. 127-144.

N. André-Pallois, L'Indochine: un lieu d'échange culturel? Les peintres français et indochinois (fin $X I X^{e}-X X^{e}$ siècle). Paris, Presses de l'EFEO, 1997, Monographie $\mathrm{n}^{\circ}$ 184, 363 p., publication de thèse soutenue en 1995.

C. Herbelin, "Tocades et discours savants: historiographie des arts de l'Indochine en situation coloniale ", Oissila Saadia et Laurick Zerbini (dir.), De la construction du discours colonial, Paris, Karthala, 2009, p. 125-148.

N. Taylor, Painters in Hanoi: An Ethnography of Vietnam, Honolulu, HI, University of Hawai’i Press, 2004.

Thái Bá Vân, « Contacts de l'art vietnamien contemporain avec l'Occident depuis la fin du xIx ${ }^{\mathrm{e}}$ siècle ", Études Vietnamiennes, 3/1992. 



\title{
13
}

\section{Le sujet lyrique dans la poésie de Han Mac Tu}

\author{
Hoai Huong Aubert-Nguyen
}

Han Mac Tu est l'un des meilleurs représentants du mouvement du Thơ Mới ("Nouvelle poésie »). Après avoir écrit des poèmes de forme classique rassemblés dans le recueil Lệ Thanh Thi Tập ("Poésies de Le Thanh »), Han Mac Tu publie en 1936 Gái Quê (" Les jeunes filles de la campagne ") salué pour sa modernité poétique. En 1938, il contracte la lèpre qui l'emportera quatre années plus tard, à l'âge de vingt-huit ans. Rongé par la maladie, il écrit Đau Thương ("Douleur d'aimer ») qui se caractérise par l'expression du désespoir et de la mélancolie, avant de parvenir à une forme de sagesse dans Xuân Như $Y^{\prime}$ (" Printemps idéal ") et Thượng Thanh Khí (" Harmonie suprême »).

Son œuvre permet d'approcher la réalité des transferts culturels franco-vietnamiens. Né au sein de la tradition classique, mais éduqué au contact de la culture occidentale, Han Mac Tu se révèle, en effet, fortement marqué par la littérature française du XIXe siècle, notamment Victor Hugo, Baudelaire, Rimbaud, Nerval... Ce qui se passe chez lui semble relever précisément du transfert et non du simple jeu d'influence.

Nous verrons dans quelle mesure la lecture de la poésie romantique française, au sein de laquelle l'émergence du sujet lyrique est un trait majeur, a permis à Han Mac Tu de développer son écriture personnelle, à travers la création d'un sujet lyrique s'adaptant à la langue et la culture vietnamiennes. Car, la mise en circulation du lyrisme dans cette œuvre conduit, non pas à une restitution à l'identique de sa forme française, mais à une appropriation, une transformation ou une resémantisation, pour reprendre des termes utilisés par Michel Espagne pour caractériser les processus de transferts culturels. 
Nous commencerons donc par caractériser le sujet lyrique de l'époque romantique, avant d'évoquer les différences entre la structure de l'énonciation en français et en vietnamien. Puis, nous étudierons l'évolution du sujet lyrique dans la poésie de Han Mac Tu.

\section{Le sujet lyrique dans la poésie romantique française}

Dans un article consacré à "L'émergence du sujet lyrique à l'époque romantique ", publié dans Figures du sujet lyrique ${ }^{1}$, Yves Tadé rappelle que le lyrisme est lié depuis son origine à la musique et au chant. Si, dans la poésie moderne, la relation avec la musicalité s'est modifiée, c'est à travers le rythme et la sonorité des mots qu'elle reste essentiellement présente. Tadé écrit qu'à l'époque du romantisme, les poètes " portent à son comble la tension entre une énonciation lyrique [...] et [...] une parole incarnée dans une voix et dans un corps ${ }^{2}$ ». C'est ainsi qu'avec les romantiques, le lyrisme devient une extériorisation de la "parole intérieure ", il devient la voix de l'intime, portée par un sujet lyrique s'exprimant dans la poésie. Ce sujet lyrique n'est pas réductible au moi biographique de l'auteur. Il se définit comme une entité complexe qui circule de locuteur en locuteur, du « je " au « tu ", du « je " à "l'univers » et du « je " à "l'autre », de sorte qu'il est constamment objet de questionnement.

En effet, comme le montre Tadé, dans la poésie romantique française, le «je " du poète peut être relié à une deuxième personne à travers l'adresse au lecteur ou à un autre allocutaire, par exemple la muse ou la bien-aimée, faisant circuler ainsi l'énonciation lyrique. Par ailleurs, pour montrer la relation entre le sujet lyrique et l'univers, Tadé écrit que le poète lyrique "se pose volontiers en interlocuteur et interprète de la nature, voire du cosmos tout entier ${ }^{3}$ ». La poésie devient ainsi la voix de la multitude des choses et de leurs secrets. On semble toucher ici à un paradoxe: comment la voix lyrique peut-elle être l'expression de l'intime et en même temps l'expression du monde extérieur? C'est que, pour les romantiques, il n'y a pas de solution de continuité entre le cœur de l'homme et l'univers. À travers la voix lyrique, peut se révéler une altérité. Comme l'écrit Rimbaud dans la lettre à Paul Demeny du 15 mai 1871, « je » est un

1. Yves Tadé, «L'émergence du sujet lyrique à l'époque romantique, in Figures du sujet lyrique (sous la direction de Dominique Rabaté), Paris, PUF, 1996, p. 11- 37.

2. Ibid. p. 12-13.

3. Ibid., p. 17. 
" autre ", au sens où il n'est pas réductible à lui-même, mais qu'il ouvre à une altérité, qui peut être différemment nommée ou interrogée, selon les auteurs. Ainsi le sujet lyrique n'est pas la caricature d'un moi égotique, mais il est le siège d'une énonciation complexe, circulant de locuteurs en figures, toujours changeant et relié au mystère d'un « autre ».

\section{La structure de l'énonciation dans la langue vietnamienne}

Pas plus que les poètes français du XIX ${ }^{\mathrm{e}}$ siècle ne l'ont fait ${ }^{4}$, Han Mac Tu ne s'est sans doute pas posé le problème du lyrisme en ces termes théoriques. Cependant, on peut émettre l'hypothèse que le romantisme français, par la nouveauté et l'étrangeté qui le caractérisaient au regard de la tradition orientale, a pu lui fournir non seulement une stimulation, mais encore un matériau et des outils poétiques qu'il a su rendre adaptables à sa propre poésie. Dans cette perspective, la question du sujet lyrique nous semble particulièrement intéressante à étudier: d'une part parce qu'elle est centrale dans le romantisme, d'autre part parce qu'elle soulève le problème de l'énonciation, qui se pose très différemment en vietnamien et en français.

En effet, dans la langue française, la structure de l'énonciation repose sur des pronoms personnels attachés à des personnes grammaticales. La première personne "je " est le locuteur, sujet de l'énonciation. La deuxième personne «tu " est l'allocutaire ou la personne à laquelle la parole s'adresse. La troisième personne "il/elle/on " désigne tout objet de pensée ou de discours, non-protagoniste de l'énonciation. En vietnamien, l'énonciation se construit tout à fait différemment. Le locuteur ou sujet de l'énonciation est variable selon sa nature propre (sexe, âge) et sa position familiale ou sociale par rapport à son interlocuteur, ou encore selon le registre de langue. Par exemple, anh désigne un homme, généralement de position supérieure par l'âge, le rang social ou familial. De plus, en vietnamien, chaque pronom est conçu à travers sa relation à une autre personne par rapport à laquelle il se définit. Le pronom anh peut ainsi être associé à différents pronoms: par exemple em... Le couple anh/em peut ainsi caractériser deux frères, ou deux amis proches, deux époux, deux amants... Or, dans tous ces cas, anh peut tenir lieu de sujet de l'énonciation tout comme d'allocutaire de l'énoncé, selon la situation. Par conséquent, dans le cadre d'une traduction en français, anh peut se

4. Comme le note Yves Tadé dans le même article, p. 16. 
comprendre comme un " je » ou un « tu " selon le contexte. On pourrait faire la même analyse des pronoms em, chị, mẹ, con, cô, chú... En vietnamien, les pronoms régissant l'énonciation sont polyvalents et variables selon les rapports entre les interlocuteurs et la situation du discours. Seuls les pronoms tôi/ta, dont nous reparlerons, font exception à la règle.

Ainsi, il apparaît que la circulation du lyrisme à la française dans la sphère culturelle du Vietnam ne saurait constituer une transposition à l'identique, tant les formes de l'énonciation sont différentes dans les deux langues. Mais elle se caractérise par une transformation et une adaptation à la langue vietnamienne, notamment à la structure énonciative reposant sur un fondement linguistique et culturel singulier. Nous pouvons étudier comment se construit le sujet lyrique dans la poésie de Han Mac Tu, avec quelles analogies, quelles différences, quelles singularités par rapport au romantisme français. Nous verrons ainsi que ce sujet lyrique est pluriel, changeant, et qu'au fil de l'œuvre, il évolue d'une forme de classicisme à une affirmation de plus en plus forte de l'individualité, avant de prêter la voix à une altérité mystérieuse et en métamorphose.

\section{Chez Han Mac Tu: le sujet lyrique de la période classique}

Les textes réunis en recueil sous le titre Lệ Thanh Thi Tập (" Poésies de Le Thanh ») témoignent de la première période de l'œuvre de Han Mac Tu. Il s'agit de poèmes de forme classique écrits en heptasyllabes et en distiques lục/bát (six/huit syllabes) inspirés de la tradition chinoise et vietnamienne. Dans ces poèmes, l'énonciation repose notamment sur la possibilité offerte par la langue vietnamienne d'utiliser la forme verbale sans pronom sujet. Ce choix contribue à façonner un sujet lyrique qui s'exprime à travers une parole retenue et mesurée.

Nous en avons un exemple dans le poème Buồn Thu, "Tristesse d'automne ${ }^{5}$ ".

Tristesse d'automne

Bredouiller, ne plus pouvoir dire une parole,

Ô l'automne, l'amour de l'automne est si désolé!

À tire-d'aile s'envole l'hirondelle,

Déjà l'on frissonne au souffle du vent roux.

Couché, impossible de réussir à rêver,

5. Han Mac Tu, Le Hameau des roseaux, traduction d'Hélène Péras et Vu Thi Bich, Orbey, Arfuyen, 2001, p. 14-15. 
Déclamer sans retenue rien que pour être un peu moins triste.

À l'infini s'étend l'ombre verte des saules,

Bientôt viendra l'hiver, les larmes montent aux yeux.

Buồn Thu

Ấp úng không ra được nửa lời

Tình thu bi thiết lắm thu ơi

Vội vàng cánh nhạn bay đi trớt,

Hiu hắt hơ may thoảng lại rồi.

Nằm gắng đã không thành mộng được,

Ngâm tràn cho đỡ chút buồn thôi.

Ngàn trùng bóng liễu trông xanh ngắt,

Cảnh sắp về đông mắt lệ vơi.

Ce texte dépeint la correspondance entre la tristesse d'un homme et la mélancolie de la saison automnale. Les formes verbales employées en vietnamien sans pronom personnel sujet sont en gras dans le texte: Ấp úng, Nằm gắng, Ngâm tràn, et on peut noter qu'au vers 4, le locuteur n'exerce pas la fonction de sujet: Hiu hắt hơi may thoảng lại rồi.

Ces expressions sont traduites en français par des infinitifs (bredouiller, dire, pouvoir, réussir, déclamer), par un participe passé (couché) et enfin au vers 4 par le pronom " on " (l'on frissonne).

Le poème donne ainsi à entendre la voix d'un sujet lyrique non exprimé. De plus, on observe l'emploi récurrent de formes négatives ou restrictives (ne plus pouvoir dire, impossible de, sans retenue, rien que pour être, un peu moins). Cet effet contribue à inscrire le sujet dans la négativité et l'absence. Le texte semble dessiner un sujet qui s'efface derrière la nature et les émotions ou sensations évoquées. En effet, les affirmations sur lesquelles repose le poème sont teintées de la mélancolie des choses qui passent et disparaissent: "l'amour de l'automne est si désolé ", " s'envole l'hirondelle ", " bientôt viendra l'hiver ", " les larmes montent aux yeux "... Le sujet lyrique s'évanouit dans une sorte de fadeur ou d'impersonnalité: il pourrait être tout homme ou bien personne. Le lecteur peut s'identifier à lui ou bien rester extérieur, et goûter simplement la musique du poème.

\section{Affirmation d'un sujet lyrique pluriel}

À partir des recueils " Les jeunes filles de la campagne " et " Douleur d'aimer ", on observe une évolution sensible du sujet lyrique: on glisse vers une expression plus vive de l'intériorité personnelle. Beaucoup de textes voient l'utilisation des pronoms tôi/ta qui désignent spécifiquement 
le « je " (dans le sens où ces pronoms sont non-polyvalents, et renvoient exclusivement au locuteur, ne pouvant désigner un allocutaire). On trouve par exemple dans le poème "Rêver " $\left(M o^{\prime}\right)$, l'affirmation d'un goût personnel, motif que l'on le trouve fréquemment dans la poésie romantique française ${ }^{6}$. À la façon dont Victor Hugo s'écrie "J'aime les soirs sereins et beaux... » ou Baudelaire: "J'aime le souvenir de ces époques nues... ", Han Mac Tu se confie dans « Rêver $^{7}$ ":

Rêver

J'aime rester debout au bord de l'étang

[...]

J'aime être assis et rêver au pied du banian

[...]

J'aime me glisser dans un fourré de roseaux

[...]

$M o^{\circ}$

Ta thích đứng lặng trên bờ ao

[...]

Ta thích ngồi mơ dưới gốc đa

[...]

Ta thích len vào trong đám lau

[...]

Dans le texte vietnamien, le poète exprime ses émotions intimes, en utilisant la première personne sujet: "J'aime "/Ta thích, repris trois fois en anaphore. Comme les romantiques, il semble confiant dans l'idée que le lecteur pourra y trouver un miroir de lui-même, s’il est vrai que le poème évoque des sentiments humains universels. Toutefois, le sujet lyrique n'est pas identifié à une personne unique: il circule d'un locuteur à l'autre.

Dans le poème "Pudeur" (Bẽn lẽn), le poème fait par exemple entendre la voix d'une jeune fille dialoguant avec la Lune, un soir de printemps $^{8}$ :

Pudeur

[...]

Ô ma sœur la Lune, mon cœur bat si fort!

6. Yves Tadé, «L'émergence du sujet lyrique à l'époque romantique, in Figures du sujet lyrique (sous la direction de Dominique Rabaté), Paris, PUF, 1996, p. 14

7. Han Mac Tu, Le Hameau des roseaux, traduction de Hélène Péras et Vu Thi Bich, Orbey, Arfuyen, 2001, p. 38- 39.

8. Ibid. p. 34-35. 
[...]

Quelle est cette voix qui parle d'amour? Comment la faire taire?

Oh! là-bas la Lune se baigne nue

Révélant sa forme d'or au fond de la ravine.

Elle laisse sans le vouloir le vent baiser ses joues

Comment rester pudique, à minuit,

J'ai peur que mon aimé ne vienne à le savoir

Et qu'il doute de ma pureté.

Bẽn lẽn

[...]

Lòng em hồi hộp, chị Hằng ơi!

[...]

Tiếng lòng ai noí? Sao im đi?

Ô kìa, bóng nguyệt trần truồng tắm

Lộ cái khuôn vàng dưới đáy khe.

Vô tình để gió hôn lên má

Bẽn lẽn làm sao, lúc nửa đêm

Em sợ lang quân em biết được

Nghi ngờ tớí cái tiết trinh em

Ce poème est important car il donne à entendre la voix d'une jeune fille de la campagne, figure centrale qui donne son titre au recueil. Dans le texte vietnamien, la jeune fille s'exprime par l'intermédiaire du pronom em, traduit par « je ", qui désigne une locutrice de position plus modeste que son interlocutrice (chị Hằng, " ma grande sœur la Lune »). Elle offre l'image d’une sensibilité féminine, initiée aux mystères de la nuit. En effet, elle entend une voix inconnue qui s'élève des roseaux, puis évoque la vision de la Lune personnifiée en amante du vent. Cette voix de jeune fille vient en écho à celle du poète, comme un relais du "je " lyrique, exprimant des émotions mêlées de pudeur, de désir, de fascination devant la nature.

Dans d'autres textes, l'expression lyrique s'inscrit dans une relation interpersonnelle entre un " je " et un " tu ", représenté par les pronoms anh/em ou tôi/em. On trouve ce schéma dans le poème Nhớ chăng, " Ne te souviens-tu pas ${ }^{9}$ ? ":

Ne te souviens-tu pas

Ne te souviens-tu pas? Tous les deux nous avons joué

Innocents comme des enfants de trois ans

9. Ibid. p. 40-41. 
J'étais debout près de toi

Je te regardais broder

Tu brodais le phénix

Posé sur une branche de firminia [...]

Nhớ chăng

Nhớ chăng? Anh cùng em nô đùa

Ngây thơ như đứa trẻ lên ba

Anh đứng bên cạnh

Coi em thêu thùa

Em thêu con phụg

Đậu trên cành ngô

Dans ce texte, écrit en vers de longueur irrégulière, on assiste au dialogue entre les pronoms anh et em traduits par " je " et « tu ». Le contexte et la convention font que la traduction choisit d'attribuer au pronom masculin anh la fonction du locuteur disant « je " et au pronom féminin em la fonction d'allocutaire représenté par le «tu ». Mais d'un point de vue strictement grammatical, la situation d'énonciation pourrait être inversée: em pourrait être désigné comme locutrice et anh comme allocutaire. Par exemple, les vers 3 et 4 :

Anh đứng bên cạnh

Coi em thêu thùa

Qui sont traduits (car on suppose que anh est locuteur):

J'étais debout près de toi

Je te regardais broder

Pourraient être traduits (si l'on suppose que em est locutrice):

Tu étais debout près de moi

Tu me regardais broder

Cela veut dire qu'en raison de la différence entre les structures de l'énonciation en français et en vietnamien, le sujet lyrique n'est pas forcément lié dans la poésie vietnamienne à un " je " identifié. L’expression lyrique repose sur différentes instances d'énonciation, qui peuvent être identifiées à une personne singulière ou à diverses personnes, pouvant être locutrices ou allocutaires. Ainsi, l'expression lyrique circule d'une personne à une autre, et chaque sujet ${ }^{10}$ est lié à un "autre " sujet l'interlocuteur par rapport auquel il se définit. Il ne peut y avoir de anh que par rapport à em ou tout autre pronom en correspondance avec lui.

10. En tant qu'« être vivant considéré dans son individualité », d'après la définition du Trésor de la langue française. 
L'expression lyrique repose ainsi sur la relation, ou le dialogue, entre ces différentes instances.

On trouve aussi ces figures en dialogue dans le poème Đôi ta ( Nous deux ») dans le recueil « Douleur d'aimer ${ }^{11}$ »:

Nous deux

Et pourtant toi et moi dans nos deux cœurs brisés

Nous avons enseveli l'image rêvée de l'aimé.

Maintenant, ici, agrippés l'un à l'autre, en ce moment même

Je sais que nous deux seuls nous sommes vraiment vivants

Échangeant de la douceur et rêvant

[...]

Alors, sans nous lasser du doux gémissement des vagues

Nous fermions les yeux, nous nous aimions comme dans la mort,

[...] Tout l'espace

Et tout le temps [...] s'anéantissent

[Au contraire de notre amour]

Đôi ta

Mà anh hay em trong tim đều rạn

Đều chôn sâu hình ảnh một người mơ.

Bây giờ đây quấn quít hiện bây giờ

Chỉ biết có đôi ta là đang sống

Đang cho nhau ngọt ngào và đang mộng

[...]

Lúc ấy sóng triều rền rĩ chưa bưa

Cứ nhắm mắt cứ yêu nhau như chết,

[...] Cả không gian

Cả thời gian [...]

Thành hư không như tình ái đôi ta...

Dans ce texte, les pronoms anh et em (traduits " toi et moi ") utilisés au premier vers laissent la place dans la suite du texte à des expressions qui unissent les deux personnes en une figure commune, réunissant le locuteur et l'allocutaire. Les expressions vietnamiennes: Đều chôn, cho nhau, yêu nhau, đôi ta (employé trois fois en donnant le titre du poème), sont ainsi traduites par le pronom "nous", par les groupes nominaux "l'un à l'autre "/ " nous deux seuls "/ " notre ", et le participe présent " échangeant ". Les deux pronoms anh et em sont ainsi unis en une entité grammaticale, à travers une énonciation lyrique liant l'un et l'autre, en une seule voix.

11. Ibid. p. $72-73$ 
C'est ainsi que l'expression lyrique s'affirme dans la poésie de Han Mac Tu comme une expression à la fois personnelle, interpersonnelle et transpersonnelle. Le sujet lyrique circule d'une personne à l'autre, chaque sujet étant défini par rapport à un " autre " sujet dans une relation ou un dialogue, au sein duquel les rôles de locuteur et d'allocutaire peuvent s'échanger, faisant ainsi circuler la parole lyrique.

\section{Le sujet lyrique et l'altérité}

Dans l'itinéraire et l'œuvre de Han Mac Tu, une importante césure apparaît entre " Les jeunes filles de la campagne " et " Douleur d'aimer "; elle correspond au moment où il contracte la lèpre. La progression de la maladie, les désordres subis par son corps et son esprit le conduisent à une profonde modification de son écriture poétique. Dans ses trois derniers recueils, les poèmes deviennent de plus en plus tourmentés et expressifs. Le sujet lyrique semble se laisse déborder par des visions étranges et une altérité inconnue, construisant une écriture qui n’est pas sans rappeler la poésie rimbaldienne. On sait que Han Mac Tu a lu Rimbaud: formulons l'hypothèse qu'il a pu y puiser des éléments féconds pour sa propre imagination.

Ainsi, l'argument du poème Rượt trăng ("Poursuivre la Lune ${ }^{12}$ ») du recueil « Douleur d'aimer " présente des analogies avec " Aube » des Illuminations. Dans les deux cas, une figure de l'altérité s’incarne dans un élément naturel personnifié: l'aube (aussi appelée la déesse) ou la Lune. Le paysage du poème "Aube " est un bois fantasmagorique qui s'anime d'une vie surnaturelle, comme la forêt sauvage du poème de Han Mac Tu. Dans les deux textes, le poète se lance à la poursuite de la divinité qui s'enfuit, jusqu'à l'image d'une chute finale. Toutefois, les différences ne manquent pas.

Dans le poème de Rimbaud, le motif de la lumière est intéressant: le texte part de la lumière blanche du matin pour traverser des paysages aux " frais et blêmes éclats " afin d'avancer vers la pleine lumière de midi. L'aube symbolise une altérité qui s’identifie à une nature harmonieuse: elle représente une forme de toute-puissance et de beauté. Le sujet lyrique s'exprime à la première personne ( "J'ai embrassé l'aube d'été »), tout en donnant, semblet-il, la parole à une troisième personne à l'avant-dernière phrase (« L'aube et l'enfant tombèrent au bas du bois. Au réveil, il était midi »), comme si le « je » avait été absorbé dans l'étreinte avec la divinité. L’expression lyrique

12. Ibid., p. 96-97. 
dit ainsi le rêve d'une immersion dans les mystères de la nature et le désir d'une union charnelle entre l'enfant-sujet lyrique et la nature.

La situation est très différente dans " Poursuivre la Lune ". D’abord, ce texte est baigné d'une lumière nocturne. Il met en scène trois protagonistes: le poète, une jeune fille et la figure de la Lune. Le poète poursuit la Lune afin de capturer ses rayons pour en faire un lit de clarté à sa bien-aimée. La Lune représente ainsi une nature que le poète rêverait de mettre au service de l'homme. Mais la divinité est jalouse, hostile aux amants, et cherche à les diviser. Le sujet lyrique circule entre le ta (" je ») du poète et le chúng tôi (" nous") représentant le couple des amants. Il exprime le désir d'une union humaine impossible, car condamnée par des forces extérieures. La déchéance de la figure lunaire du rang de puissance bienveillante à une position inférieure est illustrée par le manque de déférence avec lequel le poète s'adresse à elle. Il l'interpelle avec le simple mot trăng (lune), au lieu d'utiliser une formule plus respectueuse, comme dans le poème "Pudeur ", où la locutrice s'adresse à l'astre en l'appelant chị Hằng ( "ma grande sœur la Lune »). Ainsi, malgré certaines analogies, le texte de Han Mac Tu se révèle être à l'opposé de l'" Aube » de Rimbaud. Le sujet lyrique s’y construit pour dire une relation différente à l'altérité13.

Enfin, si l'expression lyrique donne à entendre la voix d'une altérité extérieure, nous pouvons évoquer encore le motif de la métamorphose, qui évoque la transformation du sujet lyrique lui-même en des altérités fabuleuses - une image que l'on trouve aussi notamment dans l'œuvre de Rimbaud.

Yves Tadé évoque ce motif dans un article intitulé " Métamorphoses lyriques dans la poésie en prose de Lautréamont à Michaux ${ }^{14}$ ". Il écrit au sujet des Illuminations de Rimbaud ${ }^{15}$ :

C’est cependant "Vies » et " Enfance IV », qui présentent le plus clairement [...] les postulations d'un sujet qui affirme non pas rêver d'être, mais être successivement un mystique, un savant ou un inventeur, un gentilhomme campagnard ou un simple piéton: "Je suis le saint en prières sur la terrasse [...]./Je suis le savant au fauteuil sombre [...] Je suis

13. Néanmoins, on peut noter que chez Han Mac Tu, cette figure hostile de la nature laissera plus tard la place à une altérité transcendante et bienveillante, qui conduira le poète souffrant à la rédemption à travers des poèmes empreints de mysticisme, comme dans « Ave maria » (dans « Printemps idéal ») et ou "La Lune » (dans « Harmonie suprême »).

14. Yves Tadé, "Métamorphoses lyriques dans la poésie en prose de Lautréamont à Michaux ", in Lyrisme et énonciation lyrique (sous la direction de Nathalie Watteyne), éd. Nota Bene (Québec), PU de Bordeaux (France), 2006, p. 311-327.

15. Ibid. p. 316. 
le piéton sur la grand'route [...]./Je serais bien l'enfant abandonné sur la jetée $[\ldots] »$.

Il écrit encore plus loin:

Il ne s'agit pas de comparaison ou d'analogie, comme dans le troisième "Spleen " de Baudelaire: " Je suis comme le roi d'un pays pluvieux "... Il ne s'agit pas [...] de vies imaginaires: «Je suis » n'est pas « Je m’imagine être ». Au moment où il écrit: " Je suis le saint, en prières sur la terrasse ", le sujet écrivant est vraiment, en esprit, le saint en prières sur la terrasse, - pour cesser de l'être aussitôt après. Et c'est pourquoi on peut parler de sujet lyrique, et de métamorphose lyrique. »

Dans une veine analogue, Han Mac Tu écrit dans le poème Phan Thiêt! Phan Thiêt! ${ }^{16}$ :

Phan Thiêt! Phan Thiêt!

Je me souviens des jours anciens où j'étais le Phénix [...]

La lune [...] se métamorphose en un palais somptueux.

Je suis à l'étage supérieur, je vois la mer au loin. [...]

Voilà que je partage le destin de la multitude des oiseaux [...]

Je deviens un lettré, Palais de jade et Cheval d'or,

Très généreux, très raffiné: le Poète.

Je suis un garçon plein de vitalité qui espère et qui rêve

D’une jeune fille vertueuse née au milieu du ciel suprême [...].

Phan Thiết! Phan Thiết!

Nhớ khi xưa ta là chim Phượng hoàng [...]

Trăng [...] Hóa đài điện đã rất nên tráng lệ.

Ở ngôi cao ngước mắt ra ngoài bể [...]

Là ta đây đương ở kiếp muôn chim [...]

Ta trở nên như Ngọc đàng Kim mã

Rất hào hoa rất phong vận: Người thơ

Ta là trai khí huyết ước ao mo

Người thục nũ sanh giũa trời vô thượng [...]

Dans ce poème, le sujet lyrique s'exprime à travers le pronom ta, qui occupe une place importante dans l'énonciation, puisque, comme nous l'avons dit, ce pronom renvoyait exclusivement au «je ». Ce «ta » peut être considéré, d’une part, comme le «je » de l'introspection, lorsque le locuteur se parle à lui-même. D’autre part, dans le cadre d'un dialogue, il peut aussi être pris comme le « je » utilisé par le locuteur lorsqu'il s’adresse à un allocutaire indéfini, le lecteur par exemple sur le mode de l'égalité. La

16. Han Mac Tu, Le Hameau des roseaux, traduction de Hélène Péras et Vu Thi Bich, Orbey, Arfuyen, 2001, p. 148-149. 
valeur de ce pronom permet justement à Han Mac Tu d'inscrire la plasticité du moi dans le poème: le sujet lyrique semble vidé de toute identité prédéfinie pour se fondre en diverses altérités au gré du mouvement poétique. Le « je " se rappelle son passé de Phénix, l'animal mythologique symbole d'amour et de renaissance éternelle, il redevient oiseau, puis se transforme en lettré, Poète, garçon plein de vitalité...

Dans ce mouvement, le sujet semble être projeté hors de luimême selon l'expression de Michel Collot. Dans un article consacré à cette question ${ }^{17}$, Collot émet l'hypothèse qu'une telle sortie de soi [du sujet lyrique] n'est pas l'exception, mais, pour la modernité du moins, la règle. En effet, Collot décrit cet état comme une ek-stase ou un exil. Porté par l'émotion lyrique, le sujet va à « la rencontre de ce qui le déborde du dedans comme au-dehors. [...] Si le sujet lyrique cesse de s'appartenir, c'est qu'il fait l'épreuve de son appartenance à l'autre, au temps, au monde, au langage $^{18}$ ". Dès lors, cette sortie lui permet de "s'accomplir soi-même comme un autre ${ }^{19}$ ". Ainsi, " le poème lyrique serait cet objet verbal grâce auquel le sujet parvient à donner consistance à son émotion. " On peut considérer que Han Mac Tu s'inscrit dans une certaine mesure dans cette modernité, même s’il vient d'une tradition toute différente.

Pour mieux comprendre le texte, il faut noter que Phan Thiêt est le nom de la ville où Han Mac Tu connut l'amour puis l'abandon de la femme qu'il aimait, Mông Cam, qui le quitta après avoir découvert qu'il avait contracté la lèpre. L'émotion de ce souvenir semble se traduire dans les images fantasmatiques du poème. À travers les mots, le moi sort de lui-même, se projette dans l'altérité de son être, se transformant en divers êtres fabuleux. On trouve deux fois l'expression ta là (" je suis »):

— ta là chim Phượng hoàng

- ta là trai khí huyết

Et une fois l'expression Ta trở nên nhu' (« je deviens comme »):

Ta trở nên như Ngọc đàng Kim mã

Rất hào hoa rất phong vận: Người thơ

Dans le présent du texte et son espace verbal, le sujet lyrique devient vraiment l'oiseau mythique, l'oiseau réel, le jeune homme bien portant, le lettré et la figure du Poète, à travers la réalité des émotions ressenties.

17. Michel Collot, «Le sujet lyrique hors de soi », in Figures du sujet lyrique (sous la direction de Dominique Rabaté), Paris, PUF, 1996, p. 113-125.

18. Ibid. p. 114.

19. Ibid. p. 116. 
Il semble exprimer à travers ces métamorphoses le caractère divers, insaisissable de l'homme, à la fois lui-même et tout autre.

$* * *$

Le lyrisme de Han Mac Tu puise à la source du romantisme. Comme le sujet lyrique occidental, le sujet lyrique est chez lui changeant et polymorphe. L'adaptation de l'expression poétique à la langue vietnamienne crée de profonds changements, notamment en raison de la structure de l'énonciation du vietnamien. Ainsi le sujet lyrique de Han Mac Tu, qui apparaît impersonnel dans la période classique, affirme son individualité à partir des " Jeunes filles de la campagne ". Dans ce recueil et les recueils postérieurs, la voix lyrique exprime une intériorité personnelle, circulant entre différents locuteurs, mais aussi une relation interpersonnelle et transpersonnelle, avant d'aller vers une altérité mystérieuse, révélant un être en métamorphose.

Dans l'œuvre de Han Mac Tu, les éléments occidentaux et orientaux ne semblent plus hétérogènes les uns aux autres, les premiers préexistant aux seconds, et pouvant en être détachés; mais ils s’associent et se composent, de façon à créer une forme qui possède son unité et sa singularité propres. La poésie de Han Mac Tu semble ainsi constituer un cas fécond de transfert culturel dans la littérature. Ce transfert passe par la transformation du sujet lyrique et par une nouvelle sémantisation de celui-ci. Han Mac Tu invente ainsi une voix nouvelle, avec ses perspectives et ses questionnements, participant à un véritable renouveau poétique dans le paysage littéraire vietnamien de la première moitié $d u x^{\mathrm{e}}$ siècle. 


\title{
14
}

\section{Légendes des terres sereines de Pham Duy Khiêm}

\author{
De l'art d'acclimater des contes \\ populaires vietnamiens \\ Thanh Vân Tôn Thât
}

La France et le Vietnam ont une tradition de contes et de légendes dont l'origine se perd dans la nuit des temps, histoires effrayantes ou tristes que l'on raconte aux enfants sans savoir vraiment d'où elles viennent. Écrivain vietnamien d'expression française, Pham Duy Khiêm a recueilli ces récits et leur a donné une forme littéraire en français.

Nous tenterons de mesurer dans cette expérience inédite de passage d'une langue à l'autre qui dépasse le simple exercice de traduction et d'adaptation, l'influence des modèles occidentaux, du style, du genre du conte - avec tout un arrière-plan culturel et historique - sur les récits vietnamiens ainsi revisités et mis au goût du jour pour des lecteurs français.

On observe un phénomène d'innutrition entre la littérature vietnamienne d'origine - elle-même largement inspirée par la Chine ( $\mathrm{Au}$ pays de Tê Duong en Chine [... ${ }^{1}$ ») de même que les Latins ont imité les Grecs - et la culture d'adoption française. Il convient de ne pas oublier l'occidentalisation de la langue et le début de perte d'identité asiatique après l'immense travail de transcription d'Alexandre de Rhodes des caractères vietnamiens en alphabet latin. Telle est cette œuvre

1. Pham Duy Khiêm, Légendes des terres sereines, Paris, Mercure de France, 1959 [Imprimerie Taupin \& Cie, 1943], p. 139. Toutes les citations en notes de bas de page renverront à cette édition. 
paradoxale à deux visages ou plutôt à deux voix: un auteur vietnamien évoque son pays natal en français, dans une langue autre en créant un décalage entre les deux univers. Ainsi, la matière vietnamienne ancienne revêtue de l'habit d'emprunt d'une langue étrangère se modernise et il en résulte un patchwork, un effet de métissage littéraire dans ce croisement entre d'une part, une trame vietnamienne et d'autre part, les fils, les voix, les styles occidentaux. Nous évoquerons le parcours intellectuel de l'écrivain, puis proposerons une typologie des contes avant de nous interroger sur une quête identitaire complexe et tourmentée entre tensions politiques et conflits esthétiques et linguistiques.

\section{Parcours d'un homme et premiers transferts}

Rappelons brièvement quelques éléments biographiques pour resituer Pham Duy Khiêm (né en 1908 à Hanoi, — mort en 1974, dans la Sarthe) dans son contexte historique et intellectuel. Fils de l'écrivain Pham Duy Ton et frère du compositeur Pham Duy dont les chansons sont très célèbres, il fut ambassadeur du Sud-Vietnam en France. Orphelin alors qu'il est en classe de troisième, il a appris le français dès son plus jeune âge, ce qui explique son aisance voire sa virtuosité dans une langue qui n'est plus étrangère car il se l'est appropriée dans ses moindres nuances et subtilités:

Né à Hanoi, dans la rue qui conduisait du Fleuve Rouge au Petit Lac, non loin de ce Petit Lac dont bien des Français eux-mêmes gardent un souvenir délicat, j’ai commencé à apprendre le français dans une école " franco-annamite » c'est-à-dire une école pour enfants indigènes comme moi, avec des maîtres indigènes formés directement ou indirectement par des Français ${ }^{2}$.

Au lycée Albert-Sarraut de Hanoi, il remporte tous les prix, puis, premier Vietnamien à passer le baccalauréat (en lettres classiques) il obtient une bourse qui lui permet de poursuivre ses études en France. Il éprouve le complexe du colonisé qui compense sa différence, l'écart de ses origines par le parcours d'excellence exemplaire de l'indigène doué et travailleur (à l'instar de Césaire). Pour faire ses preuves, il faut se battre sur le terrain de l'autre. À Paris, au lycée Louis-le-Grand en 1929, il est le condisciple de Pompidou et de Senghor. Premier Vietnamien à être reçu à l’École normale supérieure de Paris en 1931, il devient agrégé de grammaire en 1935 et docteur honoris causa de l'université de Toulouse

2. Pham Duy Khiêm, "Discours d'usage destiné à la séance traditionnelle de fin d'année de l'École alsacienne placée sous la présidence de M. le général d’armée Koenig », 27 juin 1964. 
en 1957. Il reçoit le prix Louis-Barthou de l'Académie française pour son roman autobiographique Nam et Sylvie (1942) publié sous le pseudonyme de Nam Kim et le prix littéraire d'Indochine en 1943 pour Légendes des Terres sereines publié la même année à Hanoi. Sa réussite passe par les études et la réussite aux concours qui lui assurent une certaine reconnaissance littéraire dans son pays d'accueil puisqu'il est édité par le Mercure de France et Plon.

Au début de la Seconde Guerre mondiale, son engagement dans l'armée française sur le territoire français comme soldat puis comme élève-officier, est mal vu par ses compatriotes. Il transpose son expérience patriotique paradoxale dans un roman épistolaire en partie autobiographique:

Il y a péril, un homme se lève - pourquoi lui demander des raisons? C'est plutôt à ceux qui se tiennent cois à fournir les raisons qu'ils auraient pour s'abstenir.

Il ne s'agit point d'un choix entre France et Annam. Il s'agit seulement de savoir la place d'un Annamite comme moi, en ce moment. Elle est ici. Et je dois l'occuper, quelque dangereuse qu'elle soit ou qu'elle doive devenir ${ }^{3}$.

De même qu'il s’interroge sur son rôle à jouer et sur son engagement politique et humain au sens large du terme, on tentera de resituer ces contes entre exotisme, folklore et universalité, considérés par l'écrivain et critique Yves Florenne dès leur parution en 1951, comme " un chefd'œuvre d’ores et déjà classique ${ }^{4}$ ». Il y a complémentarité, enrichissement pour celui qui « a la chance d'être né sur les bords du fleuve Rouge, dans un milieu sino-annamite à quoi s'était ajoutée l'influence occidentale, qui le séduisit dès leurs premières rencontres, sans jamais l'amener à renier ses sources et son sang " car ne se contentant pas d'avoir deux cultures, il a su " assimiler deux civilisations, les fondre, les dominer ${ }^{5}$ ". Cependant il apparaît parfois comme " un homme déchiré entre deux cultures, deux pays, deux fidélités ${ }^{6}$ ". L'histoire d'amour malheureuse de Nam et Sylvie traduit ses sentiments partagés et son " attachement spiri-

\footnotetext{
3. Pham Duy Khiêm, La Place d'un homme, Paris, Plon, 1958, p. 12, p. 120. Voir mon article "Point de vue (extrême)-oriental sur une guerre occidentale: La Place d'un homme de Nam Kim », revue franco-iranienne Le Pont, $n^{\circ} 2$, hiver 2007, p. 10-13.

4. Claude Cuénot, « Pham Duy Khiêm », La Table ronde, n 139-140, juillet-août 1959, p. 158-163. Voir aussi la notice de l'annuaire de l'École normale supérieure, 1976.

5. Ibid.

6. Jean-François Sirinelli, "Deux étudiants « coloniaux » à Paris à l'aube des années trente », Vingtième siècle, $n^{\circ} 18$, avril-juin 1988, p. 77.
} 
tuel à la France ${ }^{7}$ ». Pierre Laurin dans l'hommage qu'il lui rend, pense lui aussi que " partagé entre deux mondes, héritier de deux cultures (il était de fond confucéiste), déchiré en politique entre ce qu'il devait à son pays et ce qu'il devait au nôtre qui l'avait aidé à gravir les degrés de l'échelle sociale, Khiêm n'a jamais été heureux de cette double appartenance ${ }^{8}$ ". Quelle vision du monde ce recueil composite nous offre-t-il?

\section{Esquisse de classement et de typologie}

Le titre des Légendes des terres sereines est assez flou et la périphrase des terres sereines désignant le Vietnam rappelle d'autres expressions connues comme l'Empire du Milieu (ou le Pays du matin calme, p. 25). Legenda, c'est " ce qui doit être lu ", à savoir des textes à caractère exemplaire. Ces récits sont connus et racontés sans que leur forme ne soit vraiment fixée à travers différentes réécritures et versions contemporaines ${ }^{9}$. Chaque écrivain est alors moins un auteur qu'un transcripteur de récits colportés, appartenant à une tradition orale, recueillis avec des variations de détails, la trame restant similaire. La romancière contemporaine Minh Tran Huy d'origine vietnamienne écrit qu'elle a " souvent été tentée de mettre [ses] pas dans ceux de Pham Duy Khiêm ${ }^{10}$ ".

Une légende est plus longue qu'un conte, avec ses épisodes, ses péripéties; il s'agit d'un récit populaire plus ou moins fabuleux transmis par la tradition. L'arrière-plan est historique, ancré dans une réalité humaine et géographique aisément reconnaissable grâce à l'onomastique vietnamienne. Anthroponymes et toponymes contribuent à renforcer la dimension pittoresque et l'annamité de ces récits. Tel est le début de L'Histoire de Tu Thuc: "Originaire de la province de Thanh Hoa, il fut envoyé à la tête de la circonscription de Tiên $\mathrm{Du}^{11}$. " La légende vietnamienne seraitelle, comme La Légende des siècles de Hugo, "écoutée aux portes de l'Histoire ", comme le suggère l'incipit « Ceci est consigné dans les vieilles

\footnotetext{
7. Ibid., p. 85.

8. Pierre Laurin, L'Annuaire des anciens élèves du lycée Albert-Sarraut, $n^{\circ} 100$.

9. Nguyên Du, Vaste recueil de légendes merveilleuses, traduction du vietnamien et introduction et notes par Nguyên Tran Huan, collection », Connaissance de l'Orient, n 37, série vietnamienne, Gallimard/Unesco [1962], 1989.

Xuyên Lê Thi, Contes et légendes vietnamiens (avec Maurice Coyaud), Eds. Flies France, janvier 2010.

Minh Tran Huy, Le Lac né en une nuit et autres légendes du Vietnam, "Babel », Arles, Actes Sud, 2008.

10. Minh Tran Huy, op. cit., p. 12.

11. P. 39.
} 
annales $^{12}$ "? De même que dans La Légende de Saint Julien l'Hospitalier, Flaubert s'inspire du vitrail d'une église, Le Cristal d'amour associe aussi quelque peu récit et œuvre d'art, autour d'un objet magique: la tasse fabriquée à partir de la pierre translucide trouvée dans le cercueil du pêcheur " consumé par un amour sans espoir ${ }^{13}$ " après avoir été reçu par la fille du mandarin. Récit exemplaire, digne d'être lu, la légende passe de main en main, au gré des rumeurs ( La fille du mandarin apprit le prodige $^{14}$ ») comme le dicunt ou le tradunt des Latins et se métamorphose - telle la pierre puis la tasse fondue en eau au contact des larmes de la jeune fille. Cependant, se cantonnant du côté de la fiction, Pham Duy Khiêm n'a pas voulu évoquer l'histoire des deux sœurs, les Hai Ba Trung qui à l'instar de Hua Mulan en Chine et de Jeanne d'Arc en France, sont des héroïnes nationales désireuses de bouter l'ennemi hors de la patrie en danger.

La légende prend des allures hagiographiques dans La Sainte à l'enfant, avec l'ekphrasis d'une statue en bois qui rappelle une Vierge à l'enfant (p. 29): « [...] dans l'oubli où dorment les vieilles pagodes [...] la sainte est assise, son enfant dans ses bras, un sourire inaltérable sur son visage de mansuétude et de sérénité ${ }^{15}$ ". Avatar féminin de Job, cette femme malchanceuse et persécutée perd tout, chassée par son mari, rejetée par ses anciens amis, par sa propre famille, puis par la communauté des bonzes et connaît un long martyre moral. On reconnaît une forme de stoïcisme puisque cette femme supporte calomnies et privations. On pourrait relever les allusions bouddhistes au détachement par rapport aux choses humaines et terrestres (caractérisée par "sa pureté et son abnégation ", " elle était encore trop attachée à la terre ${ }^{16}$ ", voir aussi Le parfait détachement) et à la réincarnation (Les Moustiques), des références au confucianisme avec la hiérarchie entre les frères (Frères et amis, Le Bétel et l'aréquier), aux "préceptes taoïstes [...] incarnés par la figure de vieux sages magiciens ${ }^{17}$ ", mais on ne trouve pas de trace d'influence chrétienne explicite sauf dans la polysémie de "sainte ", terme qui est évidemment connoté pour un lecteur occidental. Or, le Vietnam de Pham Duy Khiêm est celui qui n'a pas connu l'occidentalisation. 
Certains textes s'apparentent au conte en tant que récits d'aventures imaginaires mêlant merveilleux et féerique avec un chronotope caractéristique qui associe distances temporelle et géographique. Pourtant c'est encore une question de point de vue tout à fait relatif, dans la mesure où l'exotisme des lieux, c'est-à-dire la couleur locale vietnamienne ne concerne que les lecteurs français, les lecteurs vietnamiens n’étant sensibles qu'à l'éloignement temporel, par exemple au début de My Châu ou l'arbalète magique: "Il y a plus de deux mille trois cents ans. " Les incipit résonnent comme ceux des contes occidentaux avec les verbes à l'imparfait et des formules comme "Autrefois ", "Il était une fois "; le cadre spatio-temporel est plus ou moins explicite mais l'excipit variable semble bien éloigné des rituelles conclusions "Ils se marièrent, vécurent heureux et eurent beaucoup d'enfants ". En effet cette formule est remplacée par des chansons ou des poèmes vietnamiens traduits en français, morale et épilogue à méditer.

Les topoï (épreuves et obstacles), les objets magiques, les types humains (les pauvres, les riches, la famille), les fonctions à la manière de Propp ou selon le schéma de Greimas sont repérables mais le conte identifié s'adresse plus à des lecteurs adultes qu'à des enfants et contrairement à la tradition occidentale de Grimm ou de Perrault — moins d'Andersen - la fin est la plupart du temps triste (« et, la voyant s'en aller, sans avoir pu lui parler, il se sentit au cœur une tristesse comme il n’en avait plus éprouvé depuis longtemps ${ }^{18}$ ") et empreinte de fatalisme. Les personnages se montrent résignés et stoïques, la littérature étant à l’image de la vraie vie, ni bonne ni mauvaise comme à la fin d'Une vie de Maupassant:

[...] contrairement aux contes de fées que ma grand-mère disait avec tant d'application, ils ne s'achevaient pas sur un happy end, mais sur une tristesse étale - un sentiment de perte étiré en point d'orgue. Ils ne niaient pas la cruauté ni l'injustice du monde, et me semblaient donc plus profonds, plus « vrais ${ }^{19}$ ".

Le recueil contient quelques récits fantastiques peuplés de revenants, marqués par des miracles (L'Enfant de la morte naît et survit dans la tombe de sa mère) et par la présence du surnaturel, les fées et les défunts faisant cependant partie du quotidien. Dans Tu Uyên ou le portrait de la Tiên, qui n'est pas pessimiste et inquiétant comme Le Portrait de Dorian Gray, la jeune fille fait penser à " Omphale " sortie de sa tapisserie pour

18. P. 167.

19. Minh Tran Huy, op. cit., p. 10. 
séduire un jeune garçon chez Théophile Gautier, immortelle et irrésistible elle aussi: "Il vit un vieillard qui vendait des images. Tu Uyên les regarda et découvrit un portrait fidèle de la jeune fille. [...] Il surprit Tiên la jeune descendue de son portrait en train de se parer $^{20}$." Dans Les Moustiques une femme veut récupérer les trois gouttes de sang qui lui rendront la vie, variation autour du motif du vampire et de la créature féminine qui meurt, ressuscite puis disparaît pour la seconde fois comme dans La Morte amoureuse de Gautier, dans une perspective nécrophile ou de triomphe de l'amour plus fort que la mort tant que la femme reste fidèle. Cependant le modeste cultivateur qui obtient d'un génie bienveillant la résurrection de sa bien-aimée, aussi humble que le pêcheur amoureux de la fille du ministre (Le Cristal d'amour) n'a rien d'un Orphée jaune à la recherche de son Eurydice annamite...

Ce sont d'autres mythes, à savoir des récits fabuleux au sens allégorique plus ou moins crypté, qui occupent une place importante. Dans ce mouvement de retour aux origines, individuel (pour l'auteur) et collectif (à l'échelle de tout un pays), une explication du monde et de la vie est esquissée: l'histoire du Crabe Da-Tràng que Minh Tran Huy rapproche du mythe de Sisyphe ${ }^{21}$, l'apparition de la Voie lactée (Le Fleuve d'argent), la naissance du moustique, les rites anciens (Le Bétel et l'aréquier), les lieux (par exemple ce que le lecteur peut voir dans le paysage pittoresque dans La Montagne de l'attente), les êtres et les choses du monde présent à travers la mise en rapport du passé immémorial avec un autre univers plus symbolique à portée universelle ("Elle fut changée en pierre et c’est ainsi qu'on peut encore la voir, droite sur le ciel, immobile dans son éternelle attente $^{22}$ »). Cette histoire triste fait surgir le spectre de l'inceste, écho d'Edipe qui n'échappe pas non plus à son destin malgré la connaissance de la prophétie: "Si tels sont les jours et les heures de vos naissances, vous épouserez fatalement votre sœur. Rien ne pourra détourner le cours du destin ${ }^{23}$. " Les trois âges de la vie représentés par la robe évolutive des mandarins rappellent l'énigme du Sphinx résolue par ce même Edipe. Les frères ennemis amoureux de la même femme dans Le Bétel et l'aréquier rappellent les luttes fratricides de la Bible (Abel et Caïn), les injustices du début du Chat botté et la rivalité entre Romulus et Remus. Le mandarin aux réponses très normandes, incapable de juger, déclarant « Vous avez

20. P. 67-68.

21. Minh Tran Huy, op. cit., p. 13, qu'elle intitule « La Perle miraculeuse ».

22. P. 37.

23. P. 34 . 
raison " au plaignant comme au défendeur, ne ressemble en rien au roi Salomon. Quant aux deux sœurs Tâm et Cam (absentes du recueil mais insérées par Minh Tran Huy dans son livre) et au chausson perdu - on comprend mieux le critère asiatique du joli petit pied — ils rappellent la pantoufle de Cendrillon. Il en résulte un effet de reconnaissance pour le lecteur français, une connivence possible à travers le repérage des échos et des parallélismes entre les deux univers culturels.

Dans le recueil, d'autres textes courts et réalistes s'apparentent à des nouvelles et à des fables en prose. On y trouve une satire de la société dénonçant la vanité humaine très proche du genre de la fable (Le Tailleur et le mandarin, Le parfait détachement), des variations autour des figures et de la structure de la famille aux liens complexes (fratries, couples désunis), des fragments de comédie humaine présentant des rapports entre maître et serviteur, par exemple dans Le gros poisson du cuisinier. On est proche des comédies occidentales avec un jeu de narration qui en dit long sur les pouvoirs de la parole conteuse et sur l'insolence du serviteur, les derniers mots lui revenant comme à Sganarelle réclamant ses gages à la fin du Dom Juan de Molière. On relève l'effet de refrain avant la morale de l'histoire: « De peur d'être réprimandé en rentrant les mains vides, il imagina le mensonge suivant en contant à Tu San: [...] "Qui prétend que mon maître est une lumière? L'argent du marché, je l'ai entièrement perdu au jeu; j'ai alors inventé une fable, et il l'a gobée sans discernement. Qui prétend que mon maître est une lumière ${ }^{24}$ ?” ". Malgré le cadre restreint, le conteur développe parfois la psychologie des personnages et présente une galerie de portraits succincts, les types humains étant souvent associés à une profession: modeste cultivateur qui ne peut lutter contre le riche commerçant qui lui enlève sa femme légère et frivole bientôt réincarnée en moustique; fille de l'empereur, tailleur, mandarin, cuisinier, chanteuse, pêcheur, femme du joueur.

\section{Enquête littéraire du collectionneur et quête identitaire}

La rédaction des légendes vietnamiennes en français correspond à un étrange moment de vide lorsque démobilisé après l'armistice, Pham Duy Khiêm attend à Marseille son embarquement pour le Vietnam. Le projet est paradoxal: restituer son patrimoine à un pays en quête d'identité, comme Pouchkine donnant ses lettres de noblesse à la langue russe.

24. P. 153-155. 
Comme pour Césaire refusant le créole, la reconnaissance passe par la langue française et non par la langue maternelle:

J'interrogeais les compatriotes, je fouillais dans les chambres d'étudiant, dans les restaurants chinois et annamites, chez les "navigateurs " annamites: la récolte fut mince. Je cherchais aussi dans mes lointains souvenirs. Ce fut alors que du fond de ma mémoire remontèrent, entre autres images, quelques restes d'une belle légende entendue il y avait vingt ou vingt-cinq ans. Mais, en dépit de tous mes efforts, les détails ne se présentaient point. Aucun étudiant ne pouvait m’aider, quelques-uns seuls avaient de vagues souvenirs, encore plus pauvres que les miens. Cependant je me rappelais qu'il s'agissait d'une ombre, l'ombre d'une femme sur le mur $^{25}$. [...] J'avais bien retrouvé, à travers les années, l'essentiel. Mais c'était loin de suffire pour constituer un récit. Comme d'autre part j'avais rêvé seulement de "traduire" des légendes annamites, le plus fidèlement possible, de les commenter au besoin (loin de penser jamais à les raconter à ma façon, avec des détails inventés personnellement), il ne me restait qu'à attendre mon retour en Indochine, pour rechercher les textes annamites et travailler à partir de sources sûres ${ }^{26}$.

Le point de départ de cette recherche est un souvenir d'enfance ${ }^{27}$, une légende perdue qui hante l'écrivain. De manière similaire Minh Tran Huy se souvient des contes de fées (implicitement occidentaux) lus par sa grand-mère et qui sont bien éloignés des contes vietnamiens et du recueil de Pham Duy Khiêm qui l'a marquée et inspirée. Le choix est emblématique et met en abyme l'écriture et ses doubles. La légende absente peut-elle être remplacée par son ombre (en langue) étrangère sans qu'il y ait trahison et perte? Le subterfuge - faire croire que l'ombre de la mère sur le mur est celle du père parti à la guerre - est source de quiproquo et provoque indirectement le suicide de la mère, qui renvoie symboliquement à la mère-patrie - tuée ou ressuscitée par l'écrivain, fidèle pour les uns, traître pour les autres. L'entre-deux douloureux fait de lui un homme de nulle part, toujours incompris, ce que l'on retrouve dans les histoires d'amour tragiques et dans le roman autobiographique publié sous pseudonyme Nam et Sylvie ${ }^{28}$. L'interdit des contes prend ici la forme de la transgression, que cela soit à travers les amours occidentales

25. Pham Duy Khiêm, L'Ombre et l'absent, p. 19.

26. Pham Duy Khiêm, préface à La Jeune femme de Nam Xuong, Hanoi, imprimerie Taupin, 1944, p. 4-6.

27. Comme la lecture de François le Champi par la mère du narrateur au début d'À la recherche du temps perdu.

28. Nam Kim, Nam et Sylvie, Paris, Plon, 1957. 
impossibles dans le roman cité ou la destinée de ces couples maudits. En effet comme chez Giraudoux ou Aragon, il n'y a pas d'amour heureux.

Le motif du malentendu est récurrent, en l'absence de dialogue, à cause de ce silence fatal:

Nous ne sommes pas bavards - et c'est peut-être là un des rares points où le caractère français et le nôtre ne se ressemblent pas, alors que de mystérieuses affinités rapprochent nos deux peuples plus que jamais peut-être rien n'a rapproché deux peuples au monde - nous ne sommes pas bavards bien que nous soyons d'un naturel ouvert ${ }^{29}$.

Dans l'inconscient du texte une catharsis discrète est à l'œuvre. On peut rapprocher d'autres cas d'expériences littéraires tiraillées entre deux cultures. Minh Tran Huy dans la préface - déjà citée — de son recueil de légendes largement inspiré par ses lectures d'enfance, envisage elle aussi un texte-tissu destiné à panser les plaies et les secrets de famille, " une histoire bien plus cruelle » dans un processus de réécriture et de réappropriation: " recueillir, trier, recomposer les histoires que j'avais lues pour en donner une version bien à moi, qui dirait en creux qui j'étais ${ }^{30}$ ". La cicatrisation est plus difficile pour Linda Lê qui tente de renouer les fils à travers les figures des filles dans Les Trois Parques et met du temps à nommer le pays du père dans ses romans, rejetant à la fois " matrie " et langue paternelle.

Se pose la question du degré d'annamité, d'auto-exotisme, quand on est confronté à l'invention et à la fabrique du Vietnam - par quel type d'auteur et pour quel public? Le lecteur non vietnamophone a-t-il besoin d'un glossaire comme celui qui permet de mieux lire la poésie de Césaire, de notes de bas de page, de traduction des anthroponymes puisque les prénoms vietnamiens ont une signification, ou sera-t-il fasciné par l'étrangeté sonore et par les pépites affleurant à la surface du texte? Si l'auteur hésite entre couleur locale et portée universelle, c'est que l'« annamitude » tend peut-être vers l'humanisme. Pourtant il s'agit bien du Vietnam et pas d'un autre pays, sauf parfois de la Chine explicitement désignée comme source et modèle littéraire (Un ministre chinois) comme le rappelle Minh Tran Huy: «ce qui fait la particularité et la cohérence de tous ces récits, c'est le besoin d'affirmer l'identité vietnamienne ${ }^{31}$ " en envisageant toutes ses facettes aussi bien dans les realia de la vie quoti-

29. Pham Duy Khiêm, « Discours d'usage destiné à la séance traditionnelle de fin d'année de l'École alsacienne placée sous la présidence de M. le général d'armée Koenig », 27 juin 1964.

30. Minh Tran Huy, op. cit., p. 11-12.

31. P. 7. 
dienne que dans sa dimension historique et géographique, en expliquant ses fondements, ses usages et ses traditions, ses paysages et reliefs naturels, son climat, ses combats ${ }^{32}$. Dans ce livre chaleureusement accueilli par le public et la presse française - Paul Guth le considère comme un chef-d'œuvre - la tradition populaire vietnamienne est sublimée par la poésie qui s'en dégage, plus exactement une prose poétique rythmée, musicale, métaphorique et lyrique.

Si l'écrivain russe d'expression française Andreï Makine est qualifié de Tolstoï français, lui qui se définit comme le Proust des steppes, alors Pham Duy Khiêm serait-il le Perrault, le Grimm, l'Andersen ou l'Afanassiev du Vietnam? Dépassant l'opposition entre atticisme et exubérance asiatique (on songe aux traductions des Mille et une nuits et à l'écart entre celle de Galland et celle de Mardrus), l'écrivain annamite comme on disait à l'époque, n'a pas recours à un français de traduction. Il rivalise avec les plus grands prosateurs en alliant richesse et sobriété et en cultivant un genre bref et mineur avec une rare économie des moyens, comme Mérimée dans ses nouvelles. En France, les commentaires sont unanimes pour louer la pureté, la limpidité et le français classique de cet écrivain venu de contrées lointaines à défaut d'être sereines.

Tenant compte de l'attente des éditeurs et du public tout en veillant à ne pas les désorienter, il ouvre une fenêtre sur l'Extrême-Orient et a recours à l'imitation et au détournement des topö̈ familiers (incipit, excipit heureux). On remarque que chaque clôture est différente: fin ouverte (" On ne dit pas quelle fut sa sentence ${ }^{33}$ ", " comment aurait-il pu se disculper $^{34}$ ? »), conclusion triste et brutale (" Puis elle se donna la mort ${ }^{35}$.", "Son mari était mort. L’effroi fut général ${ }^{36}$. "), rarement heureuse (" Leur descendance prospère encore de nos jours dans la circonscription de Khoai "), traduction d'un poème ${ }^{37}$, retour à l'époque contemporaine de l'auteur et du lecteur ("l'encens continue à brûler dans le temple consacré au génie Chu Dông Tu ${ }^{38}$ ). Pham Duy Khiêm fuit le clinquant, l'exotisme de pacotille, la pure érudition de nature historique, culturelle ou linguistique si bien que ces récits ont une résonance et une portée uni-

\footnotetext{
32. Minh Tran Huy, op. cit., p. 13.

33. P. 75.

34. P. 138.

35. P. 131.

36. P. 141.

37. P. 92

38. P. 121.
} 
verselles. Entre utopie et uchronie, le lecteur ne sait plus dans quel lieu il est lorsqu'il plonge dans un texte métissé puisque la langue française sert à dire, à redécouvrir un Vietnam immémorial, un pays qui n’a d'existence que littéraire.

De plus, Pham Duy Khiêm ajoute une dimension personnelle (La Boîte de bétel de ma grand-mère), avec la voix du conteur, la présence du narrateur et derrière lui, de l'écrivain. Ce dernier est un traducteur, non pas au sens linguistique du terme mais en tant que passeur entre deux univers et bien que ces textes ne soient pas des traductions. Pour Minh Tran Huy la situation est différente puisqu'elle découvre La Princesse et le pêcheur et de La Légende du sel dans les deux langues: «[...] édités en version bilingue, ils étaient autant de passerelles vers ce pays auquel j’étais liée sans y avoir jamais mis les pieds ${ }^{39}$ ". Entre passé et présent Pham Duy Khiêm a recours à un diptyque avec l'histoire traditionnelle du bétel et l'évocation personnelle de sa propre grand-mère - comme chez Minh Tran Huy ou dans les Contes de ma Mère l'Oye ${ }^{40}$. L'enchâssement permet d'osciller entre la réalité contemporaine et l'univers des légendes, créant un effet de parallélisme entre la vie de l'étudiant eurasien, contemporain et ami du narrateur et la destinée de l'étudiant Tu Uyên (dans Tu Uyên ou le portrait de la Tiên).

Comment peut-on être vietnamien — ni annamite, ni indochinois avec le sentiment, la conscience de son annamitude? Les Légendes des terres sereines offrent un exemple d'assimilation, d'innutrition et d'adaptation dans la lignée de la poésie de la Renaissance, du théâtre classique français ou des fables de La Fontaine. Dépassant les clivages idéologiques qui font des mots de l'autre la langue de l'ennemi, de l'envahisseur, d'un étranger fondamentalement hostile et se forgeant une identité littéraire mixte, dans une langue impeccable et dans un style parfaitement pur, Pham Duy Khiêm montre que le français n'est pas une arme mais un outil.

« Je » ne devient pas autre et échappe au risque d'aliénation même quand il peut affirmer lui aussi: " Je n'ai qu'une langue, or ce n’est pas la mienne $^{41}$ ». Ainsi se réalise un retour à et sur soi par le détour de la langue

39. Minh Tran Huy, op. cit., p. 9.

40. Perrault jouait sur la « paternité » des contes dont l'auteur est initialement présenté comme étant son fils le petit Pierre.

41. Jacques Derrida, Le Monolinguisme de l'autre, Paris, Galilée, 1996, p. 15. 
de l'autre, comparable au choix, soit d'un instrument pour composer des morceaux, soit d'une couleur dominante sur une palette, dans ce projet de recréer ce qui lui est cher afin de capter l'âme et le génie d'un peuple: « [...] ce que je connais le moins mal, c'est-à-dire: mon pays, avec mes compatriotes, ce peuple de peine, de fatigue, de douleur ${ }^{42}$."

42. Pham Duy Khiêm, "Discours d'usage destiné à la séance traditionnelle de fin d'année de l'école alsacienne placée sous la présidence de M. le général d’armée Koenig », 27 juin 1964. 



\title{
15
}

\section{Entrecroisements littéraires}

\section{Tissage et métissage dans l'œuvre de Linda Lê}

\author{
Julie Assier
}

La littérature vietnamienne francophone est singulière à plus d'un titre. La diffusion de la langue française pendant la colonisation (18581954) a touché une partie des Vietnamiens colonisés au sein de laquelle se sont affirmés quelques écrivains ${ }^{1}$ tels que Tran Van Tung, Nguyên Tiên Lang, Pham Duy Khiêm, Cung Giu Nguyen ou encore Pham Van Ky. Ces écrivains se sont particulièrement interrogés sur la confrontation des cultures vietnamienne et française au sein de la société. Les interrogations sur l'identité et sur l'interculturalité sont donc au cœur de leur discours littéraire. Mais l'avènement du quoc ngu, le manque d'infrastructures éditoriales francophones et l'absence d'un vivier de lecteurs francophones au Vietnam ont conduit ces écrivains à s'exiler, entraînant la disparition d’une francophonie littéraire au Vietnam.

Les voix francophones vietnamiennes qui résonnent aujourd'hui sont celles des écrivain-e-s de la diaspora qui se sont installés en France, au Canada ou encore en Belgique, comme Kim Doan, Kim Thuy, ou encore Nguyên Tuyêt-Nga. Dans le concert de ces voix, Linda Lê apparaît comme une écrivaine singulière, entretenant des relations très complexes avec le Vietnam. Écrivant en français, mais refusant d'évoquer directement son pays natal, elle fait figure d'outsider. Si son œuvre commence tout juste à être diffusée au Vietnam grâce au travail du traducteur Nguyên Khánh Long (1954-2011), elle demeure une écrivaine à part des lettres vietnamiennes.

1. La production littéraire vietnamienne francophone reste restreinte malgré un renouveau notable apporté par les écrivains de la diaspora. 
En France, si elle était présentée à ses débuts comme une survivante de la guerre du Vietnam ${ }^{2}$, son origine vietnamienne s'est progressivement gommée au point qu'on la désigne désormais comme une écrivaine française. Son œuvre témoigne de cet entre-deux, mais révèle surtout les allers-retours poétiques entre la France et le Vietnam, des entrecroisements littéraires qui prouvent que le métissage est bien une affaire de nuance, " une impossibilité de séparer, de trier, de trancher, de juger, de décider ", c'est en somme " avoir affaire au multiple, au complexe ${ }^{3}$."

Après avoir retracé brièvement le parcours de l'écrivaine du Vietnam à la France et son passage d'une langue à l'autre, nous nous attarderons sur l'imbrication de ses deux cultures au sein de son écriture.

\section{Itinéraire d'une francophile vietnamienne}

Née au Vietnam en 1963, d'un père vietnamien et d'une mère dont la famille fut naturalisée française, Linda Lê grandit dans un milieu biculturel. " À la maison nous parlions un drôle de langage, un mélange de vietnamien et de français ${ }^{4}$. " Initiée à la poésie vietnamienne par son père, elle est encouragée par sa mère à apprendre le français - considéré à l'époque comme un gage de réussite - et envoyée dans une école française. Elle se passionne alors pour la langue et la littérature françaises et découvre les œuvres de Balzac et d’Hugo. Loin de considérer son bilinguisme comme un atout, Linda Lê se sent progressivement déchirée entre deux langues et entre deux cultures:

J'avais seulement conscience d'être déchirée: j’acquérais des lumières qui achevaient de me rendre différente, mais cette différence n'était pas toujours bien vécue, je creusais un abîme entre moi, tentée par tout ce que l'Occident me faisait miroiter, et mon père, très attaché à sa patrie. [...] Pour moi le soleil se levait à l'Ouest, aux yeux de mon père c'était à l'Est que s'opérait la possibilité d'un ressourcement. [...] Plus ma curiosité pour l'Europe et ses anciens parapets s'éveillait, plus j'avais le sentiment de m'éloigner de mon père, et cet arrachement [...] suscitait des remords, une indicible impression de trahison ${ }^{5}$.

Alors qu'elle délaisse l'apprentissage des grandes œuvres vietnamiennes, elle dévore tous les livres français auxquels elle a accès. Elle se

2. Pierrette Blanc, «Tendre Vampire », Le Matin, 21 mars 1987.

3. Dominique Rolland, De sang mêlé. Chronique du métissage en Indochine, Elyts, Bordeaux, 2006, p. 46.

4. Linda Lê, «Tangages », in Vietnam, le destin du lotus, Riveneuve continents, n 12, automne 2010.

5. Ibid., p. 22. 
forge ainsi une image de la France représentant « une utopie aux dimensions de la bibliothèque de $\mathrm{Babel}^{6}$. " Cette fascination pour la langue et la littérature françaises est telle qu'elle fait naître un sentiment d'étrangeté chez la future romancière:

Je me sentais déracinée dans mon propre pays. On parlait vietnamien chez moi, mais je ne savais rien de la littérature vietnamienne, je n'avais rien en commun avec les enfants de mon âge, j’ai toujours été étrangère ${ }^{7}$.

L’exil en France décidé par sa grand-mère et sa mère est ainsi appréhendé avec enthousiasme:

[...] ce fut l'exaltation qui l'emporta quand les bouleversements politiques m'obligèrent, à quatorze ans, à me séparer de mon père et à quitter le pays pour rejoindre la France. Je me dois avouer que la douleur d'être arrachée à mon père parlait moins fort que l'excitation éprouvée à débarquer dans ce pays découvert à travers les livres ${ }^{8}$.

À son arrivée en France, elle continue de parler le vietnamien malgré tout et entretient une correspondance dans sa langue natale avec son père resté au pays. À l'âge de dix-huit ans, elle décide de se consacrer uniquement au français et cesse d'écrire et de parler le vietnamien. Une décision qui n'est pas sans conséquence: elle produit chez elle un sentiment de trahison et de culpabilité, à l'origine de son écriture en français:

Il y eut un moment très lié à la langue vietnamienne comme un refus d'exhumer le passé et de céder à la nostalgie — un penchant naturel chez moi. À 18 ans, j'ai cessé complètement de parler le vietnamien, que j'avais parlé et écrit jusqu'à mon arrivée en France. J'ai ressenti une impression de trahison, mais un élan qui me poussait à jouer mon vatout; quitte à choisir, je choisirais le français. Dès lors, je me suis sentie mue par la volonté de faire un avec la langue française ${ }^{9}$.

Ce bref itinéraire linguistique permet de souligner ce conflit, ce tiraillement entre sa culture et sa langue d'origine d'un côté et la culture et la langue française de l'autre. Linda Lê conçoit son choix du français au détriment de sa langue natale comme une double trahison: trahison de ses origines mais aussi trahison envers son père, qui ne maîtrisait pas cette langue. Elle va même jusqu'à considérer son acte comme un crime voire un meurtre: "J'avais tué le mandarin mais son fantôme hantait mon

6. Linda Lê, Le Complexe de Caliban, Paris, Christian Bourgois, 2005, p. 91.

7. Genevoix, Sylvie, «Linda Lê, exigeante et solitaire », Page, n³0, septembre-octobre 1994, p. 32.

8. Ibid., p. 11.

9. Rose, Sean James, « Les dits de Linda Lê », Libération, « Livres », n 8238, $7^{\text {er }}$ novembre 2007, p. V. 
encrier ${ }^{10}$. " Elle place ainsi sa littérature " sous le signe du deuil originel » et son écriture "sous le signe de la dépossession et de la trahison ${ }^{11}$ ".

\section{Ancrage occidental, traces extrême-orientales}

Dès ses débuts, elle ancre son imaginaire dans la culture occidentale en se référant à des écrivains principalement européens, refusant d'évoquer son pays natal sous quelque forme que ce soit. En tant qu'écrivaine exilée, elle s’interroge sur son positionnement et livre ses réflexions dans un premier texte intitulé " L’haleine sonore de la patrie ", en référence à Kafka, où elle livre sa vision de l'écriture en exil et des écueils à éviter. Selon elle, "Un écrivain qui use d'une langue d'emprunt vit un exil permanent: quand il écrit, il ne fait que labourer une terre étrangère; quand il publie, il exploite une terre qui ne lui appartient pas ${ }^{12}$. " Ce sentiment d'illégitimité conduit à une volonté d'intégration qui, explique-t-elle, peut prendre deux formes: se livrer à des exercices de style ou se lancer « dans la quincaillerie de l'exil »:

Or, un écrivain ne doit ni rechercher l'intégration ni quémander une identité; il doit avant tout sauvegarder son intégrité en tant que personne humaine, sachant qu'aucun groupe ethnique, social, culturel, ne tolère que des nomades évoluent librement sans vouloir rallier le gros de la troupe. Un écrivain exilé est encore plus tenté que d'autres de trouver un point d'attache: il a perdu sa langue natale, son corps est en miettes, ses racines ne s'enfoncent plus dans la terre. Irrémédiablement dépossédé, l'écrivain exilé sait qu'en échange, ce n'est pas une langue qu'il acquiert, ni un pays qu'il conquiert, mais une solitude, sa propre solitude, qu'il rencontre ${ }^{13}$.

Prônant la rupture, Linda Lê se détache de ses origines et prend comme modèle des écrivains atypiques. Nous nous attarderons sur deux en particulier: la poétesse et romancière autrichienne Ingeborg Bachmann et le philosophe roumain Emil Cioran qui l'ont grandement influencée dans sa conception de la littérature. Dans un des ses textes de conférence, Ingeborg Bachmann affirmait:

La tâche de l'écrivain ne peut pas être de nier la douleur, d'effacer ses

traces, de mystifier en l'écartant. Au contraire, il lui faut l'admettre, et la

10. Lê, Linda, Le Complexe de Caliban, Paris, Christian Bourgois, 2005, p. 51.

11. Lê, Linda, «Littérature déplacée » in Tu écriras sur le bonheur, PUF, 1999, p. 330-332.

12. Lê, Linda, «L'haleine sonore de la patrie », Hommes et migrations, $n^{\circ} 1142-1143$, avril-mai 1991, p. 49.

13. Ibid., p. 49. 
rendre à nouveau présente, pour que nous puissions voir. Car nous voulons tous devenir des voyants. Et c'est seulement cette douleur secrète qui nous rend réceptifs à l'expérience et en particulier celle de la vérité. Nous disons très simplement et justement, quand nous atteignons cet état clair, douloureux, où la douleur devient féconde: mes yeux se sont dessillés. Nous ne le disons pas parce que nous aurions apparemment perçu une chose ou un événement, mais parce que nous comprenons ce que nous ne pouvons pourtant pas encore voir. Et l'art devrait réussir cela: que nos yeux se dessillent en ce sens ${ }^{14}$.

Or, dans son texte "Littérature déplacée ", Linda Lê reprend les propos d'Ingeborg Bachmann pour avancer sa conception de l'écriture déplacée: "La tâche serait donc d'inventer une parole qui dessille les yeux. Une parole qui donne à voir. Une parole à rebours de la rhétorique. Une parole déplacée, puisqu'elle se place au cœur de la douleur sans chercher à lénifier cette douleur avec la panacée des mots ${ }^{15}$. " On voit bien comme elle imite, approprie et intègre les paroles d'Ingeborg Bachmann à sa propre réflexion:

Bachmann me paraît être un écrivain qui descend toujours dans les profondeurs, dans les abysses. Quand je l'ai lue, avec fièvre je dois dire, j'avais la révélation d'un auteur qui était toujours sans concession, et qui était pour moi comme une sorte d'émigré de l'intérieur ${ }^{16}$.

Linda Lê s'est nourrie de cette image qu'elle a construite autour de l'écrivaine autrichienne. Dans une démarche d'imitation, d'appropriation puis de réadaptation de son discours, elle révèle à son tour sa conception de l'écriture qui s'apparente pour elle à l'exploration des viscères. Par ailleurs, elle cultive cette image d'exilée intérieure, en affirmant être un écrivain en irrémédiable exil, ajoutant que si « le mot est un peu galvaudé » [...] on vit toujours dans une sorte d'exil intérieur ${ }^{17}$ ".

La lecture du philosophe roumain Emil Cioran lui a permis de mettre des mots sur ce sentiment d'exil intérieur et sur sa sensation

\footnotetext{
14. Bachmann, Ingeborg, «La vérité est exigible de l'homme. Discours de réception du prix de la pièce radiophonique décerné par les Aveugles de guerre (1959) », Les cahiers du GRIF, nº 35, vol. 35, 1987, p. 62-65.

15. Tu écriras sur le bonheur, p. 336.

16. Ibid., p. 3.

17. Sangars, Romaric, «Linda Lê: discrète Antigone », Chronic'Art, 23 juin 2004. (http://patrick. guenin2.free.fr/cantho/infovn/lindale.htm (consulté le 26 mai 2013). Cette notion d'exil intérieur nous renvoie également au livre éponyme de Roland Jaccard publié chez PUF en 1975 et qui connut un grand succès. Il définit l'exil intérieur comme un retour sur soi, un retrait de la réalité, une incapacité d'avoir une relation avec autrui, en somme une définition qu'il applique à l'homme moderne.
} 
d'" étrangèreté ", " c'est-à-dire le fait d'être étranger partout et d'être dans un rapport d'étrangeté dans sa relation avec le monde ${ }^{18}$. " Lorsqu'il décide d'abandonner le roumain pour écrire exclusivement en français en 1947, Cioran parle de révolution et de saisissement annonciateur d'une rupture $^{19}$. C'est à cette idée de rupture que Linda Lê va adhérer:

J'avais rompu avec moi-même, mais j'avais besoin de modèles, de moralistes qui, tel Cioran, mêlaient le mysticisme et une lucidité tranchante, donnaient ses lettres de noblesse à l'inconvénient d'être né et faisaient du métèque un personnage de toutes les contradictions, et donc de tous les possibles ${ }^{20}$.

Cioran se décrit lui-même comme un transfuge, un déserteur, un renégat, un traître. Dans La Tentation d'exister, il écrit même: "Qui renie sa langue, pour en adopter une autre, change d'identité, voire de déceptions. Héroïquement traître, il rompt avec ses souvenirs et, jusqu'à un certain point, avec lui-même. " Linda Lê fera sienne cette réflexion en répétant dans les entretiens être en rupture avec son pays d'origine et étrangère au monde, au réel et à son pays d'accueil. En changeant de langue, Cioran change par là même son rapport à la langue. Son ambition n'est pas à cette époque de devenir écrivain français, mais d'user du français en métèque. Cioran réhabilite cet adjectif: celui qui change de résidence, sans pour autant gagner droit de cité dans sa demeure nouvelle devient représentatif de son positionnement en tant qu'écrivain. S'il change de langue, ce n'est pas pour se faire ailleurs un nouveau chez-soi, ni pour s’intégrer mais pour redoubler son exil:

L'expérience existentielle de l'exil brusquement, débouchait sur une expérience langagière de l'exil, un exil dans la langue. Le « sentiment des distances » qui l'obsédait depuis toujours, rencontrait là sa vérité, dans un rapport métèque à l'écriture. C'en était fini de l'évidence, de l'immédiateté, qui sont les marques ou les mamelles de la langue maternelle. Plus de transparence dorénavant: plus sûrement que toute méditation morose, la langue, à jamais étrangère, à jamais étrange, allait nourrir le malaise, le mal-être indispensables pour lui à la vie de l'esprit ${ }^{21}$.

18. Entretien personnel avec Linda Lê en août 2012.

19. Sora, Mariana, Cioran jadis et naguère, suivi de Cioran: Entretien à Tübingen, Paris, l'Herne, 1988, p. 81.

20. Le Complexe de Caliban, op. cit., p. 48.

21. Leroy, Claude, « Dracula moraliste : Cioran vampire de la langue française », in Convergences et divergences dans les littératures francophones : actes du colloque (8 au 9 février 1997), Université Paris $\mathrm{X}$ - Nanterre, Centre de recherches interdisciplinaires sur les textes modernes, Paris, L'Harmattan, 1992, p. 149. 
Écrire en français s'avère pour Cioran une entreprise difficile voire un véritable combat. "Ce que j'ambitionnais c'était ni plus ni moins que de rivaliser avec les indigènes ${ }^{22}$. "Or, quand Alexandra Kurmann demande à Linda Lê pourquoi elle s'auto-qualifie de métèque, elle répond en paraphrasant Cioran:

Cela correspond à ce que je suis, il est vrai que le mot métèque a une connotation plus péjorative pour désigner l'étranger. Je trouve que c'est comme une sorte de défi de reprendre ce mot, en lui donnant un aspect provocateur. C'est le métèque qui va s'approprier une langue qu'il n'a pas au départ, qui va chercher à maîtriser cette langue, en tout cas en faisant tous les efforts possibles pour la posséder mieux que les natifs du pays ${ }^{23}$.

Cette volonté tenace de maîtriser parfaitement le français aboutit à la création d'une langue personnelle, à la fois hybride et foisonnante. Ses romans illustrent à ce titre ce que l'on pourrait appeler une esthétique littéraire fondée sur la transgression et la subversion.

Son désir de rompre avec ses origines et de se revendiquer orpheline et apatride l'amène progressivement à se réinventer une famille idéale. Puisant dans ses lectures, elle découvre des auteurs solitaires, marginaux, fous, qui deviennent des frères et sœurs en littérature. La lecture de ses articles et de ses essais montre que son imaginaire littéraire n'a pas de frontières, mais s'ancre davantage dans la littérature européenne.

Si Linda Lê affirme ne pas connaître la culture vietnamienne, on retrouve néanmoins dans ses textes des réminiscences des légendes populaires, telles que la légende du bétel et de la noix d'arec dans Les Dits d'un idiot (1995), la légende de Ngưu Lang et Chức Nữ, séparés par le fleuve dans Les Trois Parques (1997), la légende de My Chau dans Lettre Morte (1999) ou encore la légende de l'épée restituée et celle de la jeune femme de Nam Xuong dans Lame de fond (2012). De même, si elle se défend de connaître la littérature vietnamienne, elle connaît malgré tout les classiques. Elle fait notamment référence à Cao Bá Quát, le poète rebelle à la Cour de Huê, en reproduisant ces sentences parallèles dans Les Trois Parques, sans pour autant citer l'auteur et témoigne de son intérêt pour quelques écrivains vietnamiens tels que Hoang Ngoc Phach ${ }^{24}$,

22. Cioran, Emil, «En relisant », Exercices d'admiration, Paris, Gallimard, Arcades, 1986, p. 213.

23. Kurmann, Alexandra, «An Interview with Linda Lê», Paris, 19 novembre 2010, p. 1. http:// www.igrs.sas.ac.uk/sites/default/files/files/Research\%20Centres/CCWW/Linda_Le_Interview. pdf (consulté le 26 mai 2013).

24. Linda Lê, « Amours défuntes », Le Magazine littéraire, $n^{\circ}$ 456, septembre 2006, p. 87 
Nguyên Huy Thiêp ${ }^{25}$ ou encore Pham Van $\mathrm{Ky}^{26}$. Si elle tient le Vietnam à distance, il surgit néanmoins - peut-être inconsciemment - dans ses romans par l'évocation subtile et parfois voilée de pratiques culturelles vietnamiennes telles que le culte des ancêtres, les fêtes traditionnelles ou encore les rites funéraires, particulièrement dans la trilogie consacrée à la mort du père, comme si elle retrouvait " de vieilles réactions, de vieilles idées, cachées sous le vernis européen ${ }^{27}$ ", à l'image du narrateur de Frères de sang (1947) de Pham Van Ky. Enfin quelques mots vietnamiens surgissent parfois dans ses romans, comme dans Les Trois Parques ou encore Lame de fond. L'abandon de sa langue natale ne s'est pas traduit par une amnésie totale. En effet, la langue d'origine, même enfouie dans une crypte au fond de soi, ne meurt jamais ${ }^{28}$. Son écriture conserve ainsi des traces du Vietnam, tout en cultivant un ancrage occidental.

\section{L'histoire du Vietnam à la lumière des mythes gréco-romains}

L'interaction de ses deux cultures se retrouve ainsi au cœur de son écriture qui synthétise les apports de l'Orient et de l'Occident. L'exemple le plus frappant est sans doute sa capacité à proposer une relecture de l'histoire de son pays natal, le Vietnam, à travers les mythes occidentaux. C'est le cas notamment dans son roman Les Trois Parques où elle convoque plusieurs figures mythiques telles que les Parques ou encore Cassandre $^{29}$. Ce recours au mythe lui permet d'aborder le Vietnam sous un angle inattendu et de faire surgir un questionnement profond sur l'histoire. C'est particulièrement prégnant dans sa pièce de théâtre Kriss (2004) où elle choisit d'aborder la guerre du Vietnam en reprenant la trame du mythe d'Électre. Elle substitue, en effet, Kriss à Electre, Stan à Oreste, Famke à Clytemnestre, un vétéran du Vietnam à Agamemnon et le

\footnotetext{
25. Linda Lê, «Les affamés de Nguyên Huy Thiêp », Le Monde, 25 mai 1990.

26. Linda Lê, Par ailleurs (exils), Paris, Christian Bourgois, 2014, p. 87-88.

27. Pham Van Ky, Frères de sang, Paris, Seuil, 1947, p. 99.

28. On pense à cette réflexion de Julia Kristeva: « Ne pas parler sa langue maternelle. Habiter des sonorités, des logiques coupées de la mémoire nocturne du corps, du sommeil aigre-doux de l'enfance. Porter en soi comme un caveau secret, ou comme un enfant handicapé - chéri et inutile -, ce langage d'autrefois qui se fane sans jamais vous quitter. » (Étrangers à nousmêmes, Paris, Gallimard, coll. Folio/essais », 1991 (éd. or. 1988), p. 32).

29. Cf. notre article "L'ombre obsédante de Cassandre dans l'imaginaire de Linda Lê», in Cassandre, figure mythique du témoignage et de la transmission mémorielle, sous la direction de Véronique Léonoard-Roques et Philippe Mesnard, Paris, Éditions Kimé, à paraître.
} 
shérif d'une petite ville de Californie à Egisthe. En un prologue et quatre actes, il est question du meurtre d'un vétéran de la guerre du Vietnam, surnommé le boucher des rizières, par sa propre femme et le shérif de la ville. Située quelque part en Californie, en 1983, près de dix ans après la fin du conflit, la pièce met en scène Kriss, une Électre moderne guettant le retour de son frère Stan/Oreste, censé venger la mort de son père. L'intérêt de cette pièce réside sans aucun doute dans la réappropriation du mythe qui lui permet de transmettre la mémoire de la guerre du Vietnam et d'aborder la question du pardon et du dépassement.

La pièce s'ouvre sur un prologue qui met en scène trois vétérans de la guerre du Vietnam psalmodiant des souvenirs de guerre. Ces voix qui disent tout le traumatisme de la guerre ne se répondent pas. Chaque vétéran évoque par bribes ce qu'il a vécu. Le prologue inscrit ainsi la tragédie dans l'Histoire. Souvenirs obsédants, bouffées hallucinatoires, troubles de la mémoire, accès d'angoisses, ruminations amères: les traumatismes psychiques de guerre ${ }^{30}$ se manifestent de manière violente à travers leurs discours qui résument tout le choc de la guerre.

Vétéran 1

Treize millions de tonnes de bombes

Vétéran 2

De petits salauds en guenille, qu'ils disaient, les généraux.

Vétéran 3

C'est rien qu'un bronzage dans les rizières, qu'ils disaient. Nos B52 contre des soldats de plomb.

[...]

V2

Opération Niagara ${ }^{31}$, qu'ils disaient. Une cascade de bombes sur la fleur de lotus, et la victoire est à nous.

V3

Fleur de lotus, mon œil! Plutôt un droséra en plein merdier. Et nous largués là-dedans comme des mouches. Engluées. Gobées. Broyées.

Ces entrelacements discursifs reconstituent des bribes de souvenir de la guerre du Vietnam avec son lot de désillusions et d'exactions. Les

30. Crocq, Louis, Les traumatismes psychiques de guerre, Paris, Jacob, 1999, 385 p.

31. L'opération Niagara nous renvoie à la vaste opération de bombardements aériens lancée par le général Westmoreland pour défendre la base de Khe Sanh, située au nord-ouest du Sud-Vietnam, près de deux bastions nord-vietnamiens : le Laos, à 16 km à l'ouest, et la zone démilitarisée, à 25 km au nord. Le siège de Khe Sanh (21 janvier-8 avril 1969) se déroula pendant l'offensive du Têt. 
vétérans vivent tourmentés par un passé des plus présents. Ils souffrent de reviviscences intrusives des événements traumatiques qui se manifestent par des souvenirs répétitifs.

V3

[...] Faites-la taire, cette femme qui crie parce qu'on lui a amputé les deux bras. Faites-la taire, elle sanglote parce qu'on lui a annoncé qu'avec ses seins calcinés elle ne pourra pas allaiter son bébé. Faites-la taire! Ce n’est pas moi. Ce n'est pas moi qui l'ai arrosée de napalm. Ce n'était pas moi. J'avais bien vu qu'elle ne faisait que couper de la canne à sucre.

Et plus loin: "Ce n'était pas moi. C'était la guerre, la peur, la peur et la guerre ». Les vétérans invoquent les motifs de la guerre pour justifier leurs actes.

V2

Il faut rendre la liberté à une nation en détresse, disaient les généraux. C'était notre mission de délivrer ce pauvre peuple pris entre un gouvernement de trouillards pourris jusqu'au trognon et des terroristes en guenilles qui attendaient la victoire pour le mettre en cage. »

V1, sur un ton martial

Le général dit: Il y a trois raisons de continuer la guerre, mais elles n’ont pas la même importance. À soixante-dix pour cent, il faut faire la guerre pour garantir notre réputation. À vingt pour cent, pour ne pas céder ce territoire à l'emprise d'une autre puissance. À dix pour cent, pour permettre à ce peuple de vivre mieux et plus librement. »

V3

« Non, je n'étais pas à My Lai. Je ne sais rien. Je ne me souviens de rien. »

La confusion des souvenirs met en lumière la mémoire traumatisée de ces vétérans et les enjeux de la guerre du côté américain. Aux discours fragmentés des vétérans, semble répondre la voix du chœur, représenté par des enfants entourant Kriss, dès le premier acte.

Les vétérans faisaient honte à l'Amérique

Ils avaient perdu la guerre et c'étaient des monstres. Ils étaient devenus des monstres, là-bas, dans la jungle.

Marginalisés au retour de la guerre, les vétérans américains ont inspiré la honte et le dégoût; ils sont revenus dans un contexte marqué par un malaise social et politique, généré à la fois par la défaite, mais aussi par les doutes sur l'opportunité et les objectifs de cette guerre. Il fallait à tout prix oublier cette guerre, et avec elle ceux qui y avaient combattu. "Papa dit que c'était injuste. On leur a tout mis sur le dos. On leur en voulait d'avoir perdu. On ne les acceptait plus à leur retour. » La confrontation 
de ces points de vue sur la guerre du Vietnam et ses conséquences donne naissance à un discours ambivalent où Linda Lê ne prend pas parti. Dans le deuxième acte intitulé " Le retour ", où Stan revient, mais se fait passer pour un ami et annonce à sa mère la mort de son fils, un dialogue s'instaure entre les deux personnages sur la guerre du Vietnam. La mère y était farouchement opposée. Elle manifestait à l'époque " pour la fin de la guerre et la liberté de ce peuple ". Ce à quoi Stan répond: "Le peuple vietnamien est si heureux de sa nouvelle liberté qu'il fuit en masse. [...] J'ai vu des étudiants déchirer leur carte militaire. Des vétérans rentrer tête basse. Et maintenant je vois les Vietnamiens fuir sur des rafiots. Quand la mer ne les engloutit pas, les pirates se chargent de les massacrer. » Linda Lê met en regard deux discours opposés afin de les questionner et évite tout positionnement. Cette démarche lui permet ainsi une réflexion et un questionnement toujours en formation et s'interdit de réduire l'histoire du Vietnam à une seule voix.

La pièce aborde, par ailleurs, la question du pardon. La réécriture du mythe d'Electre lui permet, en effet, de faire culminer la tension entre les personnages pour finalement aboutir à la dissolution de la tragédie. Stan refuse de décliner son identité et par là même refuse l'héritage de vengeance et de haine. "Quoi? Ce ne sera qu'une farce qui terrifie une vieille femme? "Si l'image du poignard vengeur — le kriss qui inspire le titre - plane sur la pièce comme une épée de Damoclès, il n'en sera rien. Néanmoins, Stan sème le trouble dans l'esprit de sa mère qui finit hantée par le sphex à ailes jaunes, métaphore de la culpabilité. Et quand le chœur composé d'enfants rappelle à Kriss le souvenir de son père au Vietnam, elle leur répond: "Le Vietnam? Je ne connais pas. Mon père n’y est jamais allé. » La réappropriation du mythe d'Electre permet ainsi une approche inédite de la guerre du Vietnam. Cet exemple montre bien comment s'opèrent ces transferts culturels France-Vietnam au sein de la création littéraire.

$$
* * *
$$

Si l'écrivaine ne se positionne pas en tant que passeur d'une culture à l'autre, si sa démarche ne s'inscrit pas dans une volonté de jeter des passerelles entre l'Orient et l'Occident, son œuvre et sa démarche créatrice semblent être le produit d'une hybridation. Linda Lê est une écrivaine duelle, ni d'ici ni d'ailleurs, dont l'écriture se déploie sous le signe d'une altérité fertile et inspirante. Elle tire la substance de ses textes d'un imaginaire ancré dans la culture occidentale, mais ne cesse d'interroger ses 
origines. À propos de l'écrivain vietnamien francophone Pham Van Ky, elle écrit dans son dernier essai Par ailleurs (exils):

Écrivain à double face, étranger à jamais, [il] écrivait depuis ces profondeurs où toute réconciliation avec soi-même semblait impossible, et peut-être n'était-elle pas souhaitable, car pour le romancier qu'il était, suspendu entre deux mondes, l'écriture était tout à la fois la plaie et le couteau, l'espoir d'une guérison et la certitude qu'aucun mieux-être ne serait possible tant qu'il serait entre deux eaux, car il n'était ni tout à fait le fils du Vietnam ni tout à fait un enfant de cet Occident encore à conquérir ${ }^{32}$.

Il semblerait qu'à travers ces quelques lignes, elle dessine son autoportrait en creux, celui d'une exilée " silanxieuse " - pour reprendre la belle expression de Gherasim Luca - demeurant dans l'incertitude de son identité, comme le personnage de Lame de fond (2012), perdu entre Orient et Occident.

32. Ibid., p. 88. 


\section{Une nouvelle ère du roman?}

\section{Regard sur la création romanesque dans le Vietnam contemporain Cam Thi Doan}

"Il n'y a pas, pour le moment, de forme littéraire dont le pouvoir soit aussi grand que celui du roman.»

Michel Butor, Essais sur le roman, Gallimard.

On ne cherchera pas ici à dresser un panorama ou à proposer un bilan du roman vietnamien actuel, tâche considérable car l'époque est riche en innovations et révélations, liées à l'émergence d'une nouvelle génération d'auteurs nés pour la plupart après la fin de la guerre et à la nouvelle conjoncture d'un Vietnam communiste à l'épreuve de la globalisation. On s'attachera plutôt à décrire le processus-même qui est à l'œuvre ces dernières années: le retour du roman; plus précisément encore, c'est d'un renouveau romanesque qu'il s'agit.

Cette étude qui débute par des réflexions générales permettant de situer les faits, s'appuie sur la lecture de textes contemporains dont les aspects les plus novateurs en partagent la quête formelle.

\section{Évolution d'un genre}

Le roman vietnamien, on s'en doute, n'a pas attendu le xxie siècle pour voir le jour, et même le genre plonge ses racines dans un passé très reculé. Dès le $\mathrm{II}^{\mathrm{e}}$ siècle avant l'ère commune, sous la domination chinoise, les lettrés vietnamiens écrivaient en han, d'où la naissance d'une littérature savante dominée par des contes fantastiques tels le Linh Nam chich quai ${ }^{1}$

1. Linh Nam chich quai ("Recueil des êtres extraordinaires de Linh Nam »), œuvre attribuée à Trân Thê Phap (1428 ou 1492-1493 selon les sources). 
ou le Truyên $k y$ man $l u c^{2}$ et des romans semi-historiques tels Hoang Le nhat thong chi $^{3}$.

C'est l'écriture nôm (en caractères démotiques, dérivés des sinogrammes), inventée par des lettrés patriotes vers le $\mathrm{xIII}^{\mathrm{e}}$ siècle, qui a peu à peu donné naissance à des œuvres romanesques pour atteindre son apogée aux $\mathrm{XVIII}^{\mathrm{e}}$ et $\mathrm{XIX}^{\mathrm{e}}$ siècles. Parmi les œuvres les plus remarquables en nôm s'impose le magistral Kiêu de Nguyên Du, roman en vers d'une langue limpide et harmonieuse ${ }^{4}$.

L’apparition de l'écriture romanisée, mise au point par Alexandre de Rhodes pour faciliter l'évangélisation au milieu du xvII ${ }^{\mathrm{e}}$ siècle. et appelée quôc ngu (langue nationale ${ }^{5}$ ) a fait naître deux siècles plus tard les premiers romans vietnamiens modernes dont les intrigues présentent des caractères actuels et réels, par opposition aux œuvres classiques qui mettaient en scène des aventures empruntées à une littérature étrangère ou à une époque révolue comme dans le cas de Kiêu qui, écrit au XIX ${ }^{\mathrm{e}}$, représentait la période des Ming au XvII ${ }^{\mathrm{e}}$. La modernité ${ }^{6}$ est par ailleurs

2. Truyên ky man luc ("Vaste recueil de légendes merveilleuses »), œuvre de Nguyên Dữ (14.15.). Sa traduction en français a paru aux éditions Gallimard, coll. "Connaissance de l'Orient », format poche ( $\left.n^{\circ} 37\right)$, Série vietnamienne, 1989. »

3. Hoang Le nhat thong chi ("Chronique de la dynastie des Lê »), œuvre collective du groupe Ngô văn gia phai, parue au $18^{\mathrm{e}}$.

4. Kiêu raconte l'histoire d'une jeune fille de l'échelon intermédiaire de l'aristocratie qui, pour sauver son père, victime d'une calomnie, se vend comme concubine d'un homme riche avant de connaître une longue suite de déchéances. Les sentiments amoureux de Kiêu dépassent souvent pudeur et convenance. Ils sont d'ailleurs qualifiés par les lettrés de libertins. Ce chefd'œuvre de Nguyên Du est aussi un texte très moderne par le regard attentif et dépourvu de préjugés qu'il porte sur le sujet féminin.

5. Cette nouvelle écriture a permis d'alphabétiser des masses considérables de Vietnamiens en un temps record. Entre 1898 et 1912, lors des concours de recrutement du mandarinat, les épreuves en quoc ngu et en français ont été ajoutées aux épreuves en caractères chinois. De nombreux lettrés vietnamiens ont travaillé pour le promouvoir. À la fin du XIXe, Truong Vinh Ky, directeur du collège des interprètes à Saigon, a édité en quoc ngu des romans classiques écrits en nôm et un texte de lui-même intitulé Chuyen di Bac Ky nam At Hoi ("Relation du voyage au ord du Vietnam » en 1876), le premier texte en langue romanisée d'un style vivant. Également directeur du premier journal en quoc ngu, le Gia Dinh Bao, auteur d'une grammaire et d'un dictionnaire, Truong Vinh Ky a lancé un vaste mouvement de traduction d'œuvres littéraires françaises. Des revues vietnamiennes comme Dong Duong Tap Chi ou Nam Phong ont publié des traductions de romans classiques français comme Manon Lescaut, Les Trois Mousquetaires, La Peau de chagrin, etc.

6. Il est néanmoins stérile, en raison de la complexité de la question, de s'enfermer dans le débat sur le quoc ngu comme véhicule de la modernité. D'un côté, il est clair que les lettrés réformateurs vietnamiens comme Nguyên Truong To ou Phan Thanh Gian n'ont pas écrit leurs textes en quoc ngu mais en han. De l'autre, il est difficile d'imaginer que le nôm permettrait, comme les syllabaires japonais, de faire passer la culture vietnamienne dans la modernité, car à la différence des syllabaires, le nôm était d'un usage bien moins facile, n'ayant pas été codifié. 
parallèle à une mutation générique liée au développement de l'écriture en prose. Rappelons que les romans vietnamiens anciens étaient écrits non seulement en chinois ou en nôm, mais aussi en vers, genre littéraire le plus noble aux yeux des anciens lettrés. Il est donc essentiel de souligner que pour les écrivains en quoc ngu, la prose, plus que la poésie, permet au récit de traduire les nuances de la réalité et de diversifier les thèmes romanesques ${ }^{7}$. Mieux encore, libéré des règles millénaires de la versification, le roman, qui se constitue comme un genre autonome avec ses propres lois, est dès lors apte même à faire à la poésie une concurrence ouverte.

C'est dans ce contexte que se distingue L'Histoire de Lazaro Phiên, écrit par le catholique Nguyên Trong Quan et paru à Saigon en 1887. Ce roman, sans doute le premier en quôc ngu, présente en effet plusieurs éléments nouveaux. Histoire de jalousie et de meurtre, il traite du remords, thème chrétien par excellence, en relation avec des principes moraux des Vietnamiens. Rédigé dans une langue proche du quotidien, récit en prose à la première personne, il noue une intrigue au présent et propose une subtile analyse de la vie psychologique des personnages. Autre trait remarquable: sa narration est assurée par un double " je " de deux narrateurs qui mènent chacun leur récit et ne rapportent que ce qu'ils voient de leurs propres yeux ainsi que leurs sentiments intimes. De ce point de vue, L'Histoire de Lazaro Phiên est le premier texte littéraire vietnamien où l'expression du Moi connaît un véritable développement.

La parution de L'Histoire de Lazaro Phiên a joué un rôle pionnier en donnant une forte impulsion au genre romanesque. En 1925, paru à Hanoi, le roman Tô Tâm ${ }^{8}$ de Hoang Ngoc Phach qui traite d'une histoire d'amour tragique, se construit sur une prose complexe, mêlant récits, poèmes, lettres et journal intime. Les années 1930, particulièrement fructueuses, voient naître un mouvement puissant — le Tu Luc Van Doan (Groupe littéraire autonome)-, centré sur la liberté de l'individu comme fondement d'un renouveau romanesque. Il revendique pour le sujet, à travers ses œuvres nombreuses et diverses, le droit légitime de vivre et d'aimer.

De 1954 à 1975, alors que le pays est divisé en deux, la littérature connaît deux évolutions différentes. Au Nord, étroitement surveillée par

7. Sur ce sujet, voir l'ouvrage essentiel de Bui Huy Bao, Le roman vietnamien contemporain. Tendances et évolution du roman vietnamien 1925-1945, Sai Gon, Tu sach nhan van xa hoi, 1972. 
le comité central du parti communiste, elle est considérée comme un simple instrument idéologique. Au Sud, portée par une certaine liberté d'expression et influencée par la culture occidentale en raison de la traduction massive des œuvres européennes et américaines, la création romanesque connaît un formidable élan qui a malheureusement été brisé dès avril 1975. Dès lors, le réalisme socialiste s'impose comme cadre obligé de la littérature du Vietnam réunifié. Transformés en propagandistes du régime, nombre d'écrivains renoncent à l'art et à la création, comme le constatera avec amertume Nguyên Minh Châu dix ans plus tard. Alors figure majeure de la littérature officielle, ce dernier écrit dans son fameux Requiem pour une littérature d'illustration: "Les auteurs n'ont plus de pensée, je veux dire de pensée novatrice et originale. Ils existent comme un être sans âme ou avec une âme vendue au régime. Tel est le résultat le plus grave de la littérature d'illustration ${ }^{9}$."

Dès 1986, le Dôi Moi, mouvement de renouveau économique et culturel lancé par le parti communiste sous l'influence de la perestroïka soviétique, a suscité l'émergence d'écrivains critiques à l'égard du réalisme socialiste tels Bao Ninh, Duong Thu Huong, Pham Thi Hoai, et, singulièrement, Nguyên Huy Thiêp dont les premières nouvelles ont profondément modifié le paysage littéraire. Outre ses sujets audacieux - le parricide ou la mise à nu d'une société détruite par la guerre et le communisme -, l'œuvre de Nguyên Huy Thiêp explore le Mal avec concision, ironie et dérision, au point d'être souvent taxée d'immoralité par les bien-pensants. Il n'empêche que son succès a ouvert la voie à une génération d'auteurs, de nouvellistes en particulier. Le Vietnam, pendant les deux dernières décennies du $\mathrm{xx}^{\mathrm{e}}$ siècle, a connu un âge d'or du texte court - la nouvelle, le conte, le récit, le poème -, genre apte à restituer avec rapidité les bouleversements du pays d'aujourd'hui ${ }^{10}$.

Néanmoins un phénomène se confirme depuis une dizaine d'années: la nouvelle semble peu à peu reculer au profit du roman. Le nombre

9. Le 5 décembre 1987, la revue Van Nghê publie « Requiem pour une littérature d'illustration » de Nguyên Minh Châu, figure majeure de la scène littéraire. Dans cette confession douloureuse, l'auteur met à nu sa lâcheté, celle de sa génération qui a « détruit sa propre personnalité et courbé sa plume devant le pouvoir ».

10. Ce succès était dû d'autre part à l'essor de la presse écrite au début du Dôi Moi. Par sa souplesse et sa concision, la nouvelle a en effet trouvé, un support idéal dans les journaux qui, grâce à un public nombreux, assuraient aux auteurs des revenus confortables. Dans la préface de l'anthologie intitulée Au rez-de-chaussée du paradis, récits vietnamiens 1993-2003 (Philippe Picquier, 2005), j'ai analysé le phénomène de la nouvelle. 
d'ouvrages candidats aux concours de roman augmente chaque année pour faire de celui-ci le genre dominant de la création littéraire ${ }^{11}$.

\section{Quête et conquête}

Tout se passe comme si la forme brève ne pouvait plus satisfaire le plaisir narratif, le goût renouvelé pour le roman chez les jeunes auteurs, nés pour la plupart après la fin de la guerre et désireux de plonger dans le réel complexe d'un Vietnam communiste face à la mondialisation ${ }^{12}$. Le retour du genre ne va pas sans une forte volonté d'innovation, étroitement liée à la conscience - par ailleurs justifiée - de l'incapacité des formes de récit existantes à traduire les nouvelles réalités. En dépit de la censure qui reste puissante, émerge alors une littérature de créateurs ou de rupture, pour reprendre des termes de Julien Gracq ${ }^{13}$, qui refuse de concevoir le roman comme un simple véhicule de la pensée, une arme idéologique, encore moins un art d'agrément voué à la fiction. Il doit, au contraire, être un laboratoire du récit ${ }^{14}$, c'est-à-dire un des lieux privilégiés où il forge les réflexions sur lui-même en tant que type particulier de l'énoncé littéraire, sur ses modes de fonctionnement ou les spécificités de son discours, voire l'acte d'écriture.

En premier lieu, ce qui éloigne la nouvelle génération d'auteurs de la précédente, c'est une forte prise de conscience, exprimée par un truchement spécifique, la mise en scène d’un écrivain fictif qui jette un regard critique sur la littérature: c'est un point commun des romans de Nguyên

11. Comme le prouve le nombre croissant d'œuvres candidates au concours du roman organisé tous les quatre ans par l'Union des écrivains : 176 romans en 2001, 200 en 2005, 247 en 2010. Par ailleurs, on compte de plus en plus de prix littéraires destinés à la forme longue du récit. Outre celui de l'Union des écrivains, il faut citer le prix Pour la paix de notre vie co-organisé par l'Union des écrivains et le ministère de la Police et ayant attiré 146 romans en 2011, le prix Écrire quand a vingt ans organisé par les éditions de la Jeunesse et ayant attiré 147 romans en 2014, ou encore le Prix Doan Thi Diem qui, tout récemment lancé par la compagnie privée Quang Van, attire déjà un nombre élevé de candidats. Sur ce sujet, lire l'article de Hien Nguyên, Truyện dài, bước đi vừa sức của người viết trẻ? (" La forme longue du récit, la plus adéquate à la capacité des jeunes écrivains? »), paru le 7 août 2014 dans la revue To Quoc ("Patrie »), selon lequel l'intérêt financier et le prestige social - le roman est par exemple plus susceptible que la nouvelle d'être adapté à l'écran -, sont autant des raisons qui poussent l'écrivain et l'éditeur à choisir le genre romanesque. http://www.toquoc.vn/sites/vi-vn/details/11/di-san/125280/ truyen-dai-buoc-di-vua-suc-cua-nguoi-viêt-tre-. aspx

12. Certes, la constitution vietnamienne s'ouvre encore sur la dictature du prolétariat, mais le pays a rejoint l'ASEAN en 1995 et l'OMC en 2006.

13. « Pourquoi la littérature respire mal ? » in Julien Gracq, Préférences, Corti, 1961, p.71-104.

14. Michel Butor, Essais sur le roman, Gallimard, Tel, 1955, p. 9. 
Binh Phuong ${ }^{15}$, de Nguyên Viêt $\mathrm{Ha}^{16}$ ou de Thuân ${ }^{17}$. Si le premier reprend dans À l'Origine (2004) le manuscrit de " Et l'herbe », une nouvelle inédite du vieux Phùng (le personnage de l'écrivain), il ne cesse pour autant de démystifier ce dernier. C'est avec dérision qu'il le décrit: lorsque la jeune héroïne, insatisfaite par son mari dès la nuit de noces, rejoint Phùng, l'auteur le laisse impuissant face à ses désirs, tout comme il a toujours dénoncé chez lui une certaine lâcheté devant le mal et la barbarie qui l'entourent. Dérision, mais surtout autodérision: la création de ce personnage n'est-elle pas une façon pour Nguyên Binh Phuong de mettre en garde le lecteur contre l'illusion du pouvoir de l'écrivain?

Dans Made in Vietnam (2003) ${ }^{18}$, Thuân met en scène deux personnages féminins - la journaliste Phuong et l'auteure d'un roman précisément nommé Made in Vietnam dont la protagoniste s'appelle aussi Phuong. Mais elle ne leur crée pas un lien étroit, encore moins intime: leur communication ne passe que par fax ou téléphone et lorsqu'elles se rencontrent, l'une ignore l'autre. Et là est justement la force de ce texte: s'affranchir de l'analyse psychologique et de l'imitation du réel, fondements du roman traditionnel. La littérature est constituée par son propre discours. Même impression de perplexité avec $T$. a disparu, roman à suspense où la narratrice de l'épilogue se présente comme l'auteure d'un roman justement intitulé T. $a$ disparu, fantasme sur son héroïne T. tout en refusant de la voir:

Pendant que l'Airbus 330 de la compagnie Dragon Air volait au-dessus de la mer du Sud puis de la mer Orientale reliant Hong Kong à Saigon, j'imaginais une jeune femme assise au $40^{\mathrm{e}}$ rang, à côté de la fenêtre droite, dans un avion qui avait décollé en même temps que le mien, mais au départ de Charles-de-Gaulle. Je l'ai nommée T. T. pour ne pas la confondre avec une fleur, un parfum, la neige, l'automne, l'intelligence, l'harmonie ou n'importe quel prénom féminin vietnamien. T tout court [...] T. a levé la tête mais pour la baisser immédiatement, le visage toujours caché derrière sa longue chevelure noire ${ }^{19}$.

15. Poète et romancier né en 1965, Nguyen Binh Phuong est considéré comme un des pionniers du renouveau littéraire vietnamien. II vit à Hanoi.

16. Nguyen Viet Ha, écrivain catholique, domine la scène littéraire dès son premier roman paru en 1999, Une opportunité pour Dieu. Aujourd'hui, son œuvre compte une dizaine de romans, d'essais et recueils de nouvelles.

17. Après des études à Moscou, Thuân s'est installée à Paris qu'elle quitte parfois pour Hanoi, New York ou Berlin. Après Chinatown paru au Seuil en 2009, elle a publié T. a disparu (2012), L'Ascenseur de Saigon (2013) et Paris 11 août (2014) aux éditions Riveneuve.

18. Thuân, Made in Vietnam, éd. Van Moi, Californie, 2002.

19. Thuân, T. a disparu, roman traduit du vietnamien par Cam Thi Doan, coll. «Littérature vietnamienne contemporaine », éd. Riveneuve, 2012, p. 236. 
Comment écrire? La question anime désormais la création artistique. La qualité d'une œuvre dépend autant de sa composition, de sa sensibilité, de son langage que de son sujet. En d'autres termes, les questions fondamentales - l'individu, la société, l'histoire, les enjeux de la mondialisation — n'excluent nullement des expérimentations formelles. Celles-ci permettent au romancier, il est vrai, de trouver " un moyen de forcer le réel à se révéler ${ }^{20}$ ". Très précisément, Chinatown (de Thuân) ${ }^{21}$ portant sur l'exil, Khmer Boléro (de Do Khiem) ${ }^{22}$ sur l'errance, À l'origine (de Nguyên Binh Phuong) ${ }^{23}$ sur la guerre et la folie, utilisent des structures fortes, comparables à celles de la poésie ou de la musique. À l'origine est composé comme une pièce de théâtre à trois parties. Lorsque la partie $B$, la plus longue, est consacrée au " Récit ", la partie A — "Biographies » — d'une durée de trois pages, présente ses personnages, numérotés de 1 à 18, principaux et secondaires confondus, hommes et animaux unis, et surtout de manière étrange: " 4. Madame Nheo: son corps est comme une racine de ginseng. Venue s'installer au village de Linh Son avec Madame Lien. Signes particuliers: néant. Morte de maladie » (p. 13) ou «16. Le hibou: plumage couleur de fleur d'abricotier, quarante centimètres d'envergure. Bec crochu, pointu. Blessé à 11 h 15. S'envole à 12 h 00. On ne sait pour où » (p. 15). Enfin, la partie C, appelée "Epilogue ", comporte six pages, divisées en deux sous-parties — « Euvre de Monsieur Phung " et " Des rêves ». Cette dernière est fragmentée à son tour en "Les rêves de Tinh » et " Les rêves de Hiên ", lesquels, détaillés en " la nuit du 5 juin ", " la nuit du 30 juin de l'année suivante ", " La nuit du 23 d'une autre année », etc., n’ont aucun lien apparent entre eux. Que cette structure exprime-t-elle, sinon la signification profonde de l'œuvre - l'éclatement du monde?

Par ailleurs, la nouvelle création romanesque, douée d'une étonnante aptitude à explorer l'être et le monde, intègre les éléments les plus hétérogènes: documents bruts, vers, description, narration, analyses sociologique, économique ou politique, réflexion philosophique, etc. De ce point

\footnotetext{
20. Michel Butor, op. cit, p. 17

21. Thuân, Chinatown, roman traduit du vietnamien par Cam Thi Doan, coll. « Cadre vert », Seuil, 2009.

22. Né au Vietnam en 1955, Dô Khiêm alias Do Kh., vit entre Paris et la Californie après Beyrouth où il s'est marié. Poète, romancier, essayiste et scénariste, il compte parmi les écrivains les plus novateurs de la littérature vietnamienne actuelle. Ses deux premiers romans, Khmer Boléro et Saigon Samedi, écrits directement en français, ont paru aux éditions Riveneuve (en 2013 et 2014).

23. Nguyên Binh Phuong, À l'Origine, roman traduit du vietnamien par Danh-Thành Do-Hurinville, coll. « Littérature vietnamienne contemporaine », éd. Riveneuve, 2014.
} 
de vue, Une opportunité pour Dieu ${ }^{24}$, écrit en 1999 par Nguyên Viêt Ha, incarne une démarche pionnière. Euvre majeure sur la jeunesse d'aujourd'hui qui, confrontée à une impasse matérielle et morale, se réfugie dans l'exil, l'argent, l'alcool ou la mort, Une opportunité pour Dieu ne cesse de fasciner par la richesse de ses sujets - la religion et la désillusion, l'amour ou le renoncement au bonheur, l'amitié et la vanité des choses humaine. Mais ce roman séduit avant tout par son art qui réside en la mise en jeu des frontières génériques. Véritable atelier où se côtoient de multiples techniques narratives - récit à la troisième personne, journal fictif, lettre, pastiche, mise en abyme, essai, récit dans le récit —, l'œuvre propose aussi une grande palette de tons: humour, ironie, tendresse, burlesque, dérision, autodérision. Il est en effet capable d'absorber tous les genres et tous les discours pour se transformer en une œuvre d'art aussi élaborée que singulière.

D’autres aspects non moins cruciaux sont au cœur du débat formel - l'inspiration autobiographique et l'interaction des arts. C'est dans ce sens que le premier roman du peintre Vu Dinh Giang ${ }^{25}$, Parallèles, paru en 2008 à Saigon, mérite une attention particulière. Si l'œuvre dépeint les relations entre de jeunes amants homosexuels, c'est leur univers psychique et esthétique que l'auteur décrit. Là réside sa grande originalité car d'autres textes contemporains, traitant du même sujet, s’intéressent davantage à leur conflit avec la société. L'intérêt du roman se situe également dans sa forme: il se présente comme un journal intime mené à plusieurs voix dont l'identité n’est jamais déterminée. Ses personnages, désignés comme des codes ou des sigles — Gg, H, Kan — parlent d'eux-mêmes avec une telle incertitude qu'on doute de leur existence: "j'appartiens aux régions obscures ", " je suis propriétaire de la solitude ", " au fond de moi-même, je suis un insensible ", "En fait, très peu de gens savent réellement qui je suis. " Par ailleurs, pour nous faire pénétrer dans un monde onirique, intuitif, obsessionnel, dans l'inconscient à l'état pur des êtres à la recherche du plaisir et au bord de la folie, Vu Dinh Giang crée un langage qui se rapproche de la peinture abstraite, comme l'expriment ces premières lignes du roman:

Je représente les personnages secondaires et très secondaires. Les rôles secondaires, à la base, n'ont pas de visage concret - une foule invi-

24. Nguyên Viêt Ha, Une opportunité pour Dieu, roman traduit du vietnamien par Cam Thi Doan, coll. « Littérature vietnamienne contemporaine », éd. Riveneuve, 2013.

25. Né en 1976 et vivant à Ho Chi Minh Ville, Vu Dinh Giang est l'auteur de deux romans et de plusieurs recueils de nouvelles. Il est également éditeur et plasticien. 
sible. Oui, nous sommes une masse invisible, mais nous sommes tout de même nombreux. En fait, la masse ne saurait créer de force menaçante telle qu'on peut se l'imaginer. Nous ne sommes qu'une flamme à l'éclat intermittent, vacillant au gré du vent. Si nécessaire, on allume la mèche. Lorsqu'on ne veut plus de nous, nous sommes piétinés jusqu'à l'extinction. Alors, lecteur, ne soyez pas incommodé de nous voir surgir de nulle $\operatorname{part}^{26}$.

Perpétuellement à la recherche de sujets inconnus et de formes inédites, le roman contemporain est aussi incarné par Blogger ${ }^{27}$ de Phong Diêp ${ }^{28}$, paru en 2008, où Internet, jusque-là cantonné dans un simple rôle de moyen de rédaction ou de diffusion, est devenu une véritable matière littéraire. Isolée de par son origine provinciale, mise à l'écart par ses collègues, rejetée par son fiancé, contrainte d'avorter, l'héroïne de Blogger, une jeune employée d'une société d'État à Hanoi, mène son existence entre son bureau ennuyeux et sa chambre minuscule. Pour tromper son désarroi, elle ne cesse de naviguer sur Internet qu'elle pirate grâce à la connexion wifi de l'immeuble voisin. Introvertie dans la vie réelle mais reconnue comme hot blogger, elle réussit donc la communication lorsque celle-ci n’implique pas une présence physique. Tout se passe comme si son succès était conditionné par la disparition de son corps féminin perpétuellement mis à mal, victime de pauvreté, d'actes sexuels violents, d'avortement, jusqu'à l'autodestruction puisqu'elle finit par se suicider. Éclate sous une lumière crue le paradoxe d'un Vietnam encore marqué par le confucianisme et le communisme, mais mis aujourd'hui à l'épreuve de la Toile, qui autorise une certaine forme de liberté. Blogger montre précisément que la schizophrénie latente dans laquelle vivent ses personnages a pour origine cette tension permanente entre le réel et le virtuel et que, pire, ce trouble n'a d'autre issue que la mort. Ne soyons donc pas surpris que le roman de Phong Diêp, lieu d'interrogations sur le numérique et son impact social, prenne la forme d'un blog avec des entry où la confusion des auteurs est la plus grande et qui ont pour titre des termes tirés du vocabulaire internet - Blogger, Copy and paste, Chat 1 , Chat 2, Comment, Entry pour H.

26. Vu Dinh Giang, Parallèles, roman traduit du vietnamien par Yves Bouillé, coll. «Littérature vietnamienne contemporaine », Riveneuve Editions, 2014, p12.

27. Phong Diep, Blogger, roman traduit du vietnamien par Nguyên Phuong Ngoc, coll. "Littérature vietnamienne contemporaine », éd. Riveneuve, 2014.

28. Vivant à Hanoi, Phong Diep est une figure marquante de la nouvelle vague d'écrivains nés après la guerre. Elle est l'auteure du recueil de nouvelles Delete publié en 2013 aux éditions Riveneuve. 
Le travail sur la langue est donc au cœur de la recherche romanesque contemporaine. Dans Chinatown de Thuân où l'héroïne et narratrice, une Vietnamienne coincée dans une station de métro à Paris en raison d'une alerte de sécurité, se laisse emporter par rêveries, souvenirs et fantasmes, il s'agit moins de narrer une intrigue palpitante que de trouver une forme originale à son récit. Texte sans chapitre ni paragraphe dont le bloc n'a pas d'autre but que celui de restituer le flux de la conscience du personnage, le roman s'ouvre avec: «Ma montre indique dix heures [...] le métro s'arrête " et se referme sur "On attend toujours la police spéciale qui examinera le bagage abandonné [...] Ma montre indique midi. »

Ces deux heures passées sur le rail immobile suffisent pour que la narratrice nous conduise d'un bout à l'autre de la planète, du Hanoi communiste au Moscou de la Perestroïka, en passant par le Paris à l'aube du $\mathrm{xxI}^{\mathrm{e}}$ siècle. Nouement et dénouement, autant de critères fondamentaux $\mathrm{du}$ mécanisme dramatique, sont donc délaissés au profit du mouvement de la pensée. De même, la cadence accélérée des phrases juxtaposées sans lien apparent, traduit la précipitation du monde tel qu'il apparaît dans le labyrinthe mental:

Vinh se rendort la tête contre mon épaule. À douze ans, il est aussi grand que Thuy quand celui-ci en avait seize. Il déjeune à la cantine. Une assiette de purée de pomme de terre. Un steak. Deux tranches de jambon. Deux morceaux de fromage. Yaourt. Glace. Gâteau. Thuy déjeunait chez lui. Dès son retour de l'école, il faisait la cuisine pour lui et ses deux jeunes sœurs. Deux portions de riz, une poignée de liserons d'eau, six crevettes. Vinh est aussi grand que Thuy quand celui-ci avait seize ans. Comme Thuy, il a des cheveux courts. Comme Thuy, il a les yeux bridés. Ses camarades l'appellent le Chinois. Dans la rue, les gens l'appellent le Chinois. Dans le treizième arrondissement, on lui parle cantonnais. À l'école, on appelait Thuy le Chintoc. Le compatriote de Deng Xiaoping. Le larbin de Pékin ${ }^{29}$.

Do Kh, quant à lui, jubile avec des mots, multiplie ses clins d'œil et émaille ses textes de références. Dans Saigon Samedi qui, écrit directement en français, met en scène la métropole du Sud, un jour de janvier 1975, à la fin de la guerre du Vietnam où des tirs de roquettes se mêlent à la musique des pistes de dancing, l'auteur évoque Céline " cette histoire, heureusement elle se passe beaucoup la nuit ", s'amuse avec Duras et Hô Chi Minh:

L'Amant (nous allons l'appeler ainsi en hommage à la Dame de Sa Dec), naviguait les nids-de-poule avec la sérénité qui seyait à sa moustache

29. Thuân, Chinatown, op. cit, p.7-8. 
bien taillée. Signe de distinction dans un pays où la population mâle est imberbe avant d'atteindre le rang vénéré d'“ Oncle », l’homme était en civil, ce qui était un autre signe extérieur non négligeable toujours dans un pays où quasiment toute la population mâle était en armes.

Cet humour mêlé de désinvolture est à interpréter comme une volonté de lever des tabous et par-delà, de bousculer les catégories du poétique et du prosaïque, du noble et du vulgaire. Reconnaissons donc en Do Kh. l'un des rares écrivains ayant réussi à décomplexer le sexe. Voici comment il blague avec Sartre là où l'on attend le moins le très sérieux philosophe:

« Tu aurais pu la faire tourner pendant la danse [...] Et tu lui plaques la main directement sur le con! « À l'envers, mon ami, à l'envers!

— C'est ce que j'aurais dû faire, soupira Hung.

— Mais ce n'était pas pour moi. « L'envers... L'envers, c’est les autres ${ }^{30}$ ! ».

Il faut lire dans cette pratique de l'intertextualité — au sens large du terme - dont usent avec perfection Thuân et Do Kh., autant un refus de la ligne droite qu'un profond désir, celui de naviguer entre plusieurs langues, entre différentes cultures. De roman en roman, ces deux écrivains de l'exil dessinent la nouvelle configuration du monde, tel qu'il se présente depuis la fin de la guerre froide, la chute de l'Union soviétique et la montée en puissance de la Chine. Dans Khmer Boléro de Do Kh., deux anciens amants immigrés à Paris qui se retrouvent par hasard à Bangkok, partent ensemble vers l'Est, en direction du Vietnam, sans jamais l'atteindre. Les romans de Thuân, quant à eux, sont des carnets d'errance comme le suggèrent leurs titres - Chinatown, L'Ascenseur de Saigon, T. a disparu - où on déambule entre Hanoi, Saigon, Paris, Moscou, Pékin, Pyongyang, Séoul. Tout se passe comme si pour Thuân et Do Kh., leurs personnages devaient quitter non seulement le Vietnam, terre natale, mais aussi la France, pays d'adoption. À la recherche d'un nouvel ordre du monde? Peu probable car ce dernier est dorénavant décentré, et ils en sont conscients puisque précisément leurs héros ne sont pas nostalgiques sans être pour autant héroïques. Chez Thuân et Do Kh., le voyage n’est jamais initiatique.

La vision complexe que la nouvelle génération d'auteurs porte sur le Vietnam, la France et le monde autorise, en guise de conclusion, quelques

30. Do Kh., Saigon Samedi, coll. «Littérature vietnamienne contemporaine », éd. Riveneuve, 2014. Nos citations renvoient aux pages 8, 12 et 259. 
observations sur les sources de la vitalité de leur création, en liaison avec la culture et la langue française. De même que le roman vietnamien, dans sa forme moderne, se nourrit, on l'a vu, de traductions françaises ${ }^{31}-$ de même il jouit aujourd'hui d'une attention croissante de la part du public francophone. La France compte en effet trois collections dédiées à la littérature vietnamienne contemporaine, hébergées par L’Aube, Philippe Picquier et Riveneuve. D'autre part, selon l'index translationum du site de l'Unesco, la France est le pays où les livres vietnamiens sont le plus traduits. Cet intérêt contribuera-t-il à créer une nouvelle dynamique de la production romanesque vietnamienne? Il est trop tôt pour répondre à cette question, mais il semble pertinent, à l'heure de la mondialisation, de réfléchir sur l'usage du français comme langue relais pour des traductions dans d'autres langues, et peut-être pour une universalisation de la littérature vietnamienne. C'est dans ce travail d'échange et de transfert entre la culture vietnamienne et d'autres cultures du monde que la francophonie prendra toute sa signification.

31. Constamment, les lecteurs vietnamiens manifestent une préférence marquée pour le livre français. Plusieurs milliers d'ouvrages français ont été traduits au cours des dix dernières années. À côté des auteurs classiques comme Hugo, Balzac, Dumas, on trouve des contemporains tels Céline, Pennac, Houellebecq, Modiano, Proust, etc. Par ailleurs, il est important de noter que cette traduction concerne non seulement la littérature mais aussi des auteurs français de sciences sociales - Foucault, Deleuze, Bourdieu,... Soulignons ce fait, parce qu'il est particulièrement nouveau. En effet, sous la colonisation, alors que nombre de romans français ont été traduits, les idées de Voltaire, Montesquieu, Rousseau ou Darwin sont arrivées au Vietnam via le Japon et la Chine, deux pays où se rend dès la fin du XIXe siècle une partie des lettrés vietnamiens en quête d'une nouvelle source intellectuelle et scientifique. Les représentants de la France leur ont précisément caché les ferments de la pensée française et de la civilisation occidentale. 


\section{Mémoire poétique et identité historique}

\section{Éléments d'analyse du Cambodge en voix off de Nantarayao Samputho Veronica Ntoumos}

Nous souhaitons analyser ici les transferts culturels comme outils d'approche d'une mémoire et d'une identité indicible et fuyante dans le contexte post-génocidaire du Cambodge, ce voisin du Vietnam, membre jadis d'un supposé organisme indochinois. Un regard sur l'œuvre bilingue français-khmer, Le Cambodge en voix off, de Nantarayao Samputho, auteur francophone cambodgien, permettra de faire ressortir la spécificité d'une modalité de transfert culturel sous la forme de va-et-vient entre deux langues dont l'une, le français, permet plus d'audaces que le khmer, mais aussi entre deux époques. Se pose la question de savoir comment ce perpétuel va-et-vient permet de dire et de se dire après un événement traumatique tel celui du génocide perpétré par les Khmers rouges ${ }^{1}$. Comment en effet écrire après ces atrocités? Comment encore prendre la parole après qu'un tiers de la population a été assassiné?

Après avoir présenté l'écrivain et son œuvre, il s’agira de partir à la rencontre des voix marginales qui prennent la parole dans le récit et nous convient à une découverte du monde dans sa pluralité. La dernière partie poursuivra l'enquête en mettant en évidence les modes de représentations culturelles présents dans l'écriture de Nantarayao Samputho.

1. Parti communiste cambodgien d'inspiration maoïste qui a dirigé le Cambodge de 1975 à 1979. 


\section{Contexte de l'œuvre}

Combinant son métier d'écrivain à celui de professeur, Nantarayao Samputho est actuellement professeur à l'université de Phnom Penh. Écrivant à la fois en khmer et en français, il a publié chez Funan, à Phnom Penh, en 2004, dans une édition bilingue, Le Cambodge en voix off: récit de fiction qui donne la parole à ceux qu'il désigne comme les laisséspour-compte de la société cambodgienne. De la sorte, il fait référence aux marginaux d'une société elle-même meurtrie par la période coloniale et l'auto-génocide. Ces laissés-pour-compte représentent en fait un pan essentiel de la société cambodgienne comme si la marge se faisait centre et que le centre était représenté par une minorité.

Le choix de la langue s'inscrit d'emblée dans une volonté de faire dialoguer la culture khmère et la culture française. Ainsi dans une interview parue dans Courrier international (16 mars 2006), l'auteur déclare:

Aux yeux des Cambodgiens, du gouvernement, ce que j'ai écrit pourrait être choquant, et écrire en français est une forme de protection. Je peux tout dire dans cette langue. En khmer ce n'est pas évident. ${ }^{2}$

Le livre nous emmène dans le dédale des rues de Phnom Penh où se dévoile progressivement la réalité héritière d'une histoire tumultueuse. Cette plongée labyrinthique dans un Cambodge en marge échappe à toute vision monolithique: les portraits des personnages ne sont pas lisses mais révèlent au fil des pages des esquisses, des courbes dressant non pas un portrait mais une pluralité d'instantanés photographiques, non sans rappeler les portraits dressés par Raymond Depardon et Claudine Nougaret dans leur documentaire Journal de France. Cette même subjectivité se veut revendicaton dans les portraits dressés par Nantarayao Samputho où l'errance, la solitude sont les leitmotive d'instantanés littéraires.

Le regard que l'écrivain pose sur la société cambodgienne passe par l'imaginaire, par un récit de fiction qui contrairement aux comptes rendus historiques et aux enquêtes sociologiques s'intéresse en tout premier lieu à des individus et non à des échantillons.

Le récit présente ces individus sous un ensemble de volets: fenêtres sur cours retraçant la perspective des plus démunis qui n'ont d'ordinaire pas droit de cité. Sorte de tableau sur lequel est jetée à vif la peinture, tel un Pollock, la couleur choque. Les pages se présentent sous forme de diptyques: à gauche le texte en khmer et à droite en français. La calligraphie

2. Christine Chaumeau, "Entretien de Nantarayao Samputho », Courrier international, $n^{\circ} 802$, 16 mars 2006. 
même du khmer apparaît comme une illustration poétique du texte français. Chaque chapitre se referme par une illustration comme si le lecteur était face à l'envers du tableau. La première partie poursuit un découpage chronologique où les jours de la semaine se font structure du récit. Le panel des jours se décline selon le récit d'une mendiante errant dans la ville de Phnom Penh telle une hyène à la recherche de chair. Dans la seconde partie, le découpage des jours laisse place à des portraits ancrés dans une réalité bien concrète de la société khmère: un marchand de préservatifs, une belle de nuit, un passant philosophe et un futuriste. Les voix de ces laissés-pour-compte dressent ainsi le tableau d'un Cambodge contemporain porteur d'une lourde histoire qui y laisse des traces.

\section{Fiction et histoire: mode de représentation polyphonique}

Faire une lecture du Cambodge en voix off en termes de transferts culturels amène à se poser différentes questions: ce récit de fiction dans lequel se mêlent les voix de différents personnages peut-il représenter la réalité culturelle cambodgienne? Cette fiction dit-elle le vrai ou le faux? A-t-elle un poids tout aussi considérable que des comptes rendus journalistiques, sociologiques ou anthropologiques? Les deux points de vue (le compte rendu scientifique et la fiction) à leur façon cherchent le vrai. La vérité en littérature, ce serait la prise de conscience que la réalité est inépuisable et l'enjeu littéraire serait d'imaginer toujours de nouveaux points de vue sur elle.

Ces points de vue dans l'écriture de Nantarayao Samputho s’inscrivent dans une tension entre la réalité - témoignages recueillis dans les rues de Phnom Penh - et un plan fictionnel, la mise en intrigue. Le récit produit ainsi des symboles en identifiant le passé qui ouvre un espace singulier au présent.

Quels sont dès lors les modes de représentations dont use la fiction? Dans Le Cambodge en voix off ils sont pris en charge par une multiplicité de voix subalternes. En recueillant les voix du peuple, le livre se veut un roman polyphonique: de la voix du marchand de préservatifs à celle d'une prostituée en passant par celle d'un philosophe de la rue, l'écriture se décline de façons diverses. Nantarayao Samputho absorbe ainsi divers discours qui s'hybrident à l'intérieur de l'œuvre: feuilleté de voix qui s'entremêlent et qui empruntent les voies multiples et sinueuses de la réalité cambodgienne. La lecture qui conviendrait relèverait d'une écoute bakhtinienne. Il s'agit d'appréhender l'œuvre non plus en considérant 
un point de vue unique de l'auteur sur la réalité cambodgienne, mais en écoutant la voix de personnages multiples. Ceux-ci ne sont plus uniquement des personnages-sujets mais des êtres en dialogues dont les paroles résultent des interrelations entre personnages. Chacun a ses propres lunettes et par cette confrontation de discours, l'auteur ne veut pas imposer au lecteur une conclusion ou une synthèse mais s'inscrit davantage dans une sorte de dialogue. La photographie de la réalité cambodgienne ne résulte pas d'un seul point de vue, mais se construit au contraire grâce aux imbrications de discours divers: la réalité " naît entre les hommes qui la cherchent ensemble, dans le processus de leur communication dialogique $^{3}$ ". Ce dialogue socratique sur la société cambodgienne permet à Nantarayao Samputho de cultiver les contrastes et de remettre en question les idées reçues. Par le choix d'une écriture polyphonique il adhère au refus du peuple cambodgien de se dépeindre de manière monolithique.

La question posée par ces mises en voix, c’est la manière de se représenter la délicate réalité d'un pays qui renaît progressivement de ses cendres. Nantarayao Samputho refuse de dresser un portrait statique de la société cambodgienne car celle-ci fluctue de la trace d'un douloureux passé - qui veut à la fois être oublié et pourtant si présent - vers une conjoncture du présent qui bâtit un futur marqué par des ruptures. Chaque génération a son moment de finitude suivi d'une période à réinventer. Moment de période génocidaire sous le régime de $\mathrm{Pol} \mathrm{Pot}^{4}$ et de ses camarades suivi d'une période à réinventer, celle du post-traumatisme. Tout est à recréer et en littérature aussi quand l'on sait qu'avant 1975 le Cambodge comptait deux cents écrivains et qu'à la fin du régime des Khmers rouges seuls quatre d'entre eux ont survécu. On mesure à quel point il existe un creuset entre l'avant génocide et la période qui a suivi.

\section{Douloureuse réinvention}

Pour comprendre ces transferts d'une période de massacres à celle qui suit, quelques balises historiques se révèlent nécessaires. Si la volonté des Khmers rouges lors de leur entrée à Phnom Penh le 17 avril 1975 était

3. Mikhaill Bakhtine, Poétique de Dostoïevski, Paris, Le Seuil, 1970, p. 155.

4. Pol Pot, de son vrai nom Saloth Sâr (1925-1998), Premier ministre du Cambodge (le Kampuchéa démocratique) de 1976 à 1979. Après des études en France de 1949 à 1952 où il fréquente le cercle marxiste des étudiants khmers, il sera le leader révolutionnaire du régime des Khmers rouges. 
d'en finir avec deux mille ans d'histoire et de faire table rase, il s'agissait avant tout de mettre en place la naissance de l'homme nouveau entièrement soumis à la collectivité et à l'État.

Façonner une nouvelle société signifiait pour Pol Pot et ses camarades à la fois vider la capitale ainsi que toutes les villes du pays de ses habitants, coupables, selon eux, d'embourgeoisement mais surtout une rupture radicale et nécessaire avec le passé et l'étranger. Afin de mettre en place cet homme nouveau, les familles ont été dispersées, les meurtres sont devenus pratique courante.

Comment affronter ensuite l'écriture dans un pays mal remis de l'oppression où la démocratie figure certes dans les textes, mais non dans les pratiques? Comment libérer les esprits de l'autocensure?

Par l'usage d'écrits métaphoriques, par la polyphonie sans point axial, l'écriture de Nantarayao Samputho s'efforce de rendre vie à une littérature assassinée par la révolution, marquée par la destruction des livres et la mise à mort des intellectuels en vue de permettre au lecteur de frayer son chemin dans le dédale d'un Cambodge en reconstruction.

Les mots, les images, les films, peuvent-ils se faire témoin de l'horreur? Si la fiction tant littéraire, picturale que cinématographique ${ }^{5}$ ne s'attache pas à dépeindre de manière scientifique et objective les atrocités du passé, elle parvient toutefois à transmettre l'émotion du vécu. Pour faire comprendre la manière dont se comporte la société cambodgienne, ce qu'elle fait et les raisons pour lesquelles elle agit de la sorte, Nantarayao Samputho a choisi de représenter cette société, de se la représenter, c'est-à-dire de l'imaginer.

Dans cette perspective, on peut se demander si pour arriver à dire l'indicible - dans ce cas particulier du Cambodge ou l'horreur fut, dans une large mesure, également une affaire de transfert et où l'identité khmère est restée comme affolée, hors d'elle-même - le recours au transfert ne se révèlerait-il pas nécessaire? Ce passage d'une langue à l'autre, d'une période génocidaire à la période suivante, du traumatisme à l'écriture, ne serait-il pas un moyen privilégié de revendiquer une relation spécifique à la réalité?

\section{Transferts culturels, sociaux et graphiques}

Dans Le Cambodge en voix off, la culture est envisagée à travers une logique relationnelle. Cette perspective permet d'envisager l'individu,

5. Dont notamment le documentaire franco-cambodgien, L'image manquante, primé à Cannes en 2013 et réalisé par Rithy Panh. 
non pas à partir de ses caractéristiques, mais à partir d'un réseau ou sous forme de rhizome. Cette logique relationnelle ne laisse pas de côté le lecteur mais au contraire l'investit dans les questionnements des personnages. Ainsi, lorsque le personnage de la mendiante s'interroge sur son identité en se comparant à un arbre chétif, elle s'adresse directement au lecteur:

Je suis comme un arbre chétif. La lune et les étoiles se chamaillent. Quand il pleut, il tombe des trombes. Tout est inondé, l'arbre frissonne. Quand il fait chaud, on brûle l'arbre, l'arbre étouffe, il s'effeuille. Comment voulez-vous que cet arbre prospère alors que la nature ferme les yeux? Même quand il pleure, à qui crier sa souffrance? Je suis semblable à un édifice sans fondation, un arbre sans racines. Jusqu'à quand pourra-t-il tenir droit? Un simple souffle suffit pour le renverser! Comment voulez-vous qu'il donne des fruits, s'il n'a aucune chance ${ }^{6}$ ?

En ayant recours à la métaphore de l'arbre, l'écriture dévoile au lecteur la dure nécessité de trouver sa place dans une société dont les racines mêmes sont non seulement asséchées par les atrocités du passé mais aussi arrosées actuellement en vain par l'élite cambodgienne. À qui crier sa souffrance dans un monde de sourd? Comment communiquer? Tout comme le Cri noir du Cahier d'un retour au pays natal', le cri cambodgien est celui d'un peuple dont l'identité a été bafouée. Face à ce peuple sans racine, face à ce peuple qui étouffe, qui frissonne, qui n'a aucune voix dans l'histoire, se dresse l'écrivain qui s'engage à témoigner, à rendre compte par des transferts littéraires des contours d'une identité en pleine mutation.

Le lecteur est convié à suivre les réflexions du personnage de la mendiante: réflexion critique sur la société bourgeoise cambodgienne, sur la pauvreté et l'inégalité des statuts. Comme si plus rien n'avait d'importance, comme si toute dignité avait été bafouée, le personnage de la mendiante convie le lecteur à suivre son récit à travers une véritable plongée dans les pérégrinations des voies subalternes. Et personnifiant le malheur jusqu'à lui donner le statut d'interlocuteur, la mendiante lui adresse ces propos: “Tu m’as été donné en héritage dès l'âge de quatre ans. J'ai mendié en permanence pendant des mois et des mois, des années et des années jusqu'au commencement de mon déclin ${ }^{8}$."

\footnotetext{
6. Nantarayao Samputho, Le Cambodge en voix off, Phnom Penh, Funan, 2004, p. 51

7. Aimé Césaire, Cahier d'un retour au pays natal, Paris, éd. Présence africaine, 1971.

8. Ibid., p. 17.
} 
La mise en page du texte français et du texte khmer côte à côte permet au fil des phrases d'être en présence de la culture de l'autre qui dans la première partie du recueil semble unie par une réalité similaire et bouleversante qu'est la pauvreté. Cette pauvreté tragique est rendue par une écriture poétique, une musicalité du texte: musique qui produit un discours au-delà du particularisme vers un va-et-vient de l'autre à soi.

Poursuivant son récit, le personnage de la mendiante s'interroge sur les raisons de son destin: "Mais cette pauvreté d'où vient-elle? Du manque d'intelligence, de prévoyance, ou de la société qui m’écrase et m'empêche d'avoir la tête hors de l'eau'. " Cause interne ou cause externe, la question reste en suspens. Le récit nous invite à réfléchir sur la logique de reproduction sociale, dans sa dimension proprement sociale et à travers la violence symbolique qu'elle engendre. Cette violence qui s'exprime dans une langue crue et acerbe faisant écho à la violence quotidienne de la vie des marginaux au fil des rues de Phnom Penh. Le personnage de la mendiante peut-il sortir de l'habitus qui l'enchaîne à cette spirale infernale héritée de génération en génération? Ne faut-il pas également s'interroger sur le rôle des transferts sociaux qui façonnent tout autant que les transferts culturels le patchwork des identités?

$\mathrm{Au}$ fil des pages, au fil des jours évoqués dans le récit, les identités s'ancrent et s'inscrivent davantage dans une réalité cambodgienne. Ne correspondant pas au tableau prestigieux dressé par les différentes périodes de la civilisation khmère, le personnage de la mendiante évoque tour à tour les grandes étapes de la civilisation cambodgienne. Ainsi, les temples d'Angkor et leur beauté qui a traversé les millénaires; le règne du roi Norodom Sihanouk, comme époque paisible et extraordinairement prospère; la période de Pol Pot comme grande période de collectivisation sans individualisme, sans ambition, sans féodalisme; l'époque de l'Apronuc $^{10}$ comme symbole du maintien de la paix. Chacun vantant à sa manière les divers moments qui ont ponctué l'histoire du Cambodge sous forme d'apologie qu'il ne faudrait en rien entacher par un paysage de pauvreté.

Ainsi, Nantarayao Samputho en tant qu'écrivain aborde les transferts culturels avec l'outil de la littérature. Il a bien conscience des contraintes de son matériau qu'est l'écriture et en fait le vecteur d'une transposition

9. Nantarayao Samputho, op. cit., p. 57.

10. L'APRONUC est l'autorité provisoire des Nations Unies au Cambodge dont l'objectif était de faire respecter les accords de Paris de 1991 qui visaient à mettre fin à la guerre entre l'Etat cambodgien et les Khmers rouges. 
graphique, analogie entre le va-et-vient d'une culture à l'autre et le transfert graphique (va-et-vient de l'œil du texte français au texte khmer).

Le Cambodge en voix off examine les possibilités et les limites du transfert de la notion du traumatisme des Khmers rouges dans le champ littéraire, en explorant l'espace intermédiaire entre le non-dit traumatique et le dit textuel, entre témoignage et fiction.

$$
* * *
$$

Perpétuelle oscillation, constant va-et-vient envisagé comme outil d'approche d'une mémoire, l'écriture par transfert de Nantarayao Samputho permet de s'éloigner ce que l'on reprochait aux ethnographes d'autrefois: de projeter les catégories européennes sur la société cambodgienne. De même qu'on ne peut projeter nos catégories actuelles sur un passé divers et multiple, il faut s'abstenir de projeter un modèle de l'unité de la conscience individuelle sur les discours et les savoirs d'autres cultures, comme cela a bien été souvent le cas pour écrire la période des Khmers rouges. Il s'agit au contraire de redéployer notre vision, et de ressaisir les configurations dans lesquelles notre savoir est éparpillé. En proposant un double jeu d'écriture pour approcher la réalité cambodgienne, Nantarayao Samputho échappe au danger de reconstituer quelque chose comme un tissu homogène qui serait l'esprit d'une culture, d'une époque, d'une classe sociale.

Pour avoir transfert, il faut envisager des relations de recomposition qui permettent de se situer en amont des catégories figées et de remettre en cause nos concepts préconçus. Ce que Nantarayao Samputho a réussi en posant la littérature non pas comme pensée sur l'histoire, sur l'anthropologie, sur les études coloniales mais avec elles. Cette démarche permet de mettre en situation de dialogue différentes disciplines en vue d'aller chercher des solutions au sein d'autres époques et d'autres cultures. En contexte de globalisation, il est de plus en plus nécessaire de défendre ainsi les spécificités en préservant la conscience des processus de dialogue et de corrélation qui sous-tendent l'identité littéraire, historique, culturele.

Par un transfert d'imaginaire oscillant entre univers asiatique et univers français, par un transfert du traumatisme khmer, Nantarayao Samputho ouvre des voies possibles pour une écriture de l'élucidation d'une mémoire effacée. Il propose des modes d'écriture pour dire le passé, pour écouter la souffrance, pour comprendre sans juger les réalités cambodgiennes contemporaines en observant leur texture de transferts polyphoniques. 
CINQUIÈME PARTIE

$$
\begin{gathered}
\text { LES COLLECTIONS } \\
\text { VIETNAMIENNES DE LA } \\
\text { BIBLIOTHÈQUUE NATIONALE } \\
\text { DE FRANCE }
\end{gathered}
$$



18

\section{Le fonds indochinois de la Bibliothèque nationale de France}

\section{Denis Gazquez}

Fonds régional particulier, le fonds indochinois est un fonds clos regroupant des documents en vietnamien romanisé (quôc ngu) édités dans l’Indochine coloniale entre 1922 et 1954 et entrés par dépôt légal. Il fait partie des nombreux fonds concernant le Vietnam colonial présent dans les différents départements ${ }^{1}$. Il faut souligner l'importance de ces fonds: du fait de la colonisation, la France possède la plus importante documentation existant au monde sur le Vietnam colonial. Dans la documentation coloniale française, les documents édités en Indochine ou en métropole sur l'Indochine sont parmi les plus nombreux bien que la colonisation ait été relativement tardive et courte (90 ans pour la Cochinchine, 70 ans pour l'Annam et le Tonkin), et que la population française y ait toujours été très peu nombreuse. Du point de vue de la masse documentaire seule l'Algérie dépasse l'Indochine mais avec une colonisation bien plus longue et surtout une population européenne beaucoup plus nombreuse.

\section{Les fonds concernant le Vietnam colonial dans les départements spécialisés}

Le département des Estampes et de la Photographie regroupe gravures, affiches, photographies, cartes postales, images de toutes sortes (images d’Epinal, publicités, collections de particuliers,...). Des documents

1. Gazquez, Denis. Les fonds sur l'Indochine coloniale à la Bibliothèque nationale de France: esquisse, Paris, Bibliothèque nationale de France, 2012. 
sur le Vietnam sont présents dans tous ces supports avec un grand nombre de photographies, par exemple celles du Dr Hocquard des années 1883-1886, et de séries de cartes postales.

Le département des Cartes et Plans conserve à côté de cartes anciennes d'avant la conquête, des cartes manuscrites des explorateurs et des militaires. Et surtout la très nombreuse cartographie coloniale éditée essentiellement par le service géographique de l'Indochine: cartes de l'Indochine au 1/200000 publiées entre 1888 et 1894; cartes du Vietnam au1/25000 éditées entre 1902 et 1953 qui couvrent essentiellement les deltas du Mékong et du fleuve Rouge, les plus peuplés et les plus importants sur le plan économique, la série n’était pas terminée en 1954; cartes du Vietnam au 1/100 000 éditées entre 1947 et 1955.

La Société de géographie, fondée en 1821 à Paris, regroupe explorateurs et devient à la fin du XIX ${ }^{\mathrm{e}}$ siècle un puissant lobby colonial. Elle organise puis subventionne des voyages d'exploration, établit une correspondance avec voyageurs et explorateurs, organise des conférences, publie des relations inédites dans son bulletin, crée une bibliothèque d'une très grande richesse: livres, manuscrits, cartes, photographies ${ }^{2}$. Elle est en dépôt à la Bibliothèque nationale depuis mars 1942, au département Cartes et Plans. L'Indochine est très présente dans ses fonds:

- 230 manuscrits environ: rapports manuscrits inédits, correspondance de nombreux voyageurs et explorateurs avec la Société de géographie, textes manuscrits d'articles publiés dans les revues de la Société.

- des milliers de photographies, cartes postales, aquarelles et dessins, cartes manuscrites, 80 \% antérieurs à 1914, dont de nombreuses photographies sur plaques de verre en cours de traitement et de numérisation.

Dans le département des Manuscrits il y a quelques centaines de documents en caractères chinois mais aussi, dans le fonds maçonnique, la correspondance entre des loges indochinoises et le Grand-Orient de France. Quelques documents aussi au département des Arts du spectacle: programmes de théâtres et de cinémas, fête de Jeanne d'Arc, voyage de Khai-Dinh en France en 1922...

Dans le département des Médailles et Monnaies on trouve des pièces, des billets et de jetons.

2. Fierro, Alfred. Inventaire des manuscrits de la Société de géographie, Paris, Bibliothèque nationale, 1984. ; Fierro, Alfred. Inventaire des photographies sur papier de la Société de géographie, Paris, Bibliothèque nationale, 1986. ; Méhaud, Catherine. Manuscrits de la Société de géographie concernant l'Asie et l'Océanie, Paris, Bibliothèque nationale, 1979. 
Le département de l'Audiovisuel contient des enregistrements sonores hérités du musée de la Parole, dont ceux de l'exposition coloniale de 1931, des enregistrements datant de la guerre d'Indochine (Leclerc, Ho Chi Minh), des disques de musique et de chants populaires ${ }^{3}$, des films documentaires...

\section{Les fonds imprimés sur le Vietnam colonial dans les départements thématiques de Tolbiac}

La Bibliothèque nationale de France offre un ensemble particulièrement riche d'ouvrages et de périodiques imprimés.

Dès la conquête de la Cochinchine l'administration coloniale édite en français périodiques, brochures, codes juridiques... On peut dire que la presse à imprimer a accompagné le sabre de la conquête; dès 1862 est publié à Saigon le Journal officiel de l'expédition française. Les ouvrages édités en français sont nombreux et arrivent en partie à la Bibliothèque nationale, le dépôt légal s’applique aussi pour les publications éditées dans les colonies mais il est aléatoire. Les ouvrages sont traités comme ceux édités en France et recensés dans le catalogue général.

Parallèlement, il y a aussi une édition d'ouvrages en vietnamien romanisé (le quôc ngu): de 1874 à 1921 environ un millier de livres entrent dans les collections et sont intégrés dans le fonds général ${ }^{4}$.

Les imprimés en français et les périodiques en quôc ngu édités entre 1922 et 1954 continuent à être traités au fur et à mesure dans le fonds général. Par contre les livres, brochures et plaquettes en vietnamien sont mis de côté et formeront plus tard le fonds indochinois proprement dit.

Il n'existe pas de catalogue particulier pour les ouvrages français édités en Indochine mais on peut utiliser la Bibliotheca indosinica (19121932) d'Henri Cordier pour la période qui va de la conquête à 1912, et la Bibliographie de l'Indochine française de Paul Boudet pour la période 1913 à 1935, la presque totalité des ouvrages recensés sont présents à la BNF.

Quelques remarques sur les fonds imprimés:

3. Collard, Claude (ed.), Des sources pour l'histoire de l'émigration en France: guide, Paris, Bibliothèque nationale, 2006.

4. Rageau, Christiane. Les ouvrages relatifs au Vietnam dans les collections de la Bibliothèque nationale, Paris, Bibliothèque nationale, 1980. 
- On peut estimer le nombre de périodiques édités en Indochine en français et dans les langues locales à environ 1500 titres. Le catalogue de Jean-Claude Poitelon en recense 2350 mais inclut des périodiques postérieurs à l'indépendance et jusqu'à 1975;

- les ouvrages et les périodiques dont on vient de parler ne sont pas tous entrés grâce au dépôt légal: beaucoup ont été intégrés lors de la disparition dans les années 1960 après la fin de la guerre d'Algérie de nombreuses institutions coloniales qui possédaient bibliothèques et centres de documentation. Et nous continuons à enrichir nos collections sur l'Indochine coloniale en particulier grâce à des dons: par exemple nous venons de recevoir une collection unique en France du quotidien édité à Saigon par l'armée française: Indo-Soir: quotidien du soldat français en Indochine (du n $\mathrm{n}^{\circ}$, 9 mars 1946, au n 66, 8 juin 1946) et à quelques achats (par exemple des publications du $2^{\mathrm{e}}$ bureau, destinées aux officiers du corps expéditionnaire);

- Il y a aussi ce qui ne devrait pas y être, par exemple les bulletins internes de la police criminelle de Cochinchine et du Tonkin des années 1930 et 1940 avec leurs annexes de listes de condamnés, évadés, recherchés, déserteurs-insoumis;

Les recueils sont des imprimés conservés et catalogués par ensembles créés par les bibliothécaires et classés par organismes émetteurs ou par thèmes. Nous avons compté 2300 recueils de documents coloniaux français et environ le tiers concerne l'Indochine;

Ce sont des documents très variés: statuts, publicités, documents administratifs, techniques (horaires des trains, livrets de marche des conducteurs de locomotives), tracts, prospectus touristiques, documents internes d'associations, amicales...). Quelques-uns sont classés dans le fonds général: tracts vietminh, affiches de Leclerc... mais la plupart sont classés à part avec cotes WZ. Une liste des 2300 recueils coloniaux est en préparation.

\section{Le fonds indochinois: historique d'un fonds}

Je ne résiste pas au plaisir de reproduire le début de l'introduction de Christiane Rageau au catalogue du fonds indochinois:

La lueur émeraude des lampes de table de la grande salle de lecture me plaisait. Je la trouvais gaie. Pour cette raison sans doute, je préférais faire mes recherches à la Bibliothèque nationale, calme, discrète et presque déserte à l'époque. C'était en 1965 [connaissant le vietnamien elle est 
embauchée pour préparer le catalogue général en projet en s’occupant de la tranche des Trân]. Autorisée à consulter les ouvrages directement sur les rayonnages, j'étais fréquemment amenée à me retrouver à un même endroit du magasin où s'empilaient plusieurs centaines de boîtes en carton contenant des brochures plutôt que des livres rédigés en vietnamien romanisé. Parfois il s’agissait de livres plus épais, pauvres d'aspect, souvent vieillis, jaunis et bien fragiles. Il était facile aussi de constater que les paquets encore ficelés qui se trouvaient à côté des boîtes n’avaient jamais été ouverts depuis leur dépôt et contenaient le même genre d'ouvrages ${ }^{5}$.

On peut attribuer indirectement à Albert Sarraut, gouverneur général de l'Indochine puis ministre des colonies, et à Paul Boudet son directeur des archives et des bibliothèques de l'Indochine, la constitution du fonds indochinois. Si l'édition a connu un extraordinaire développement dès le XIX ${ }^{\mathrm{e}}$ siècle, c'est sans doute lié à l'adoption de l'écriture du vietnamien en lettres latines et aussi aux particularités de l'histoire de la colonisation de l'Indochine. La nouvelle écriture a aussi favorisé la démocratisation de l'enseignement. Son adoption progressive par tous: missionnaires, administration, nouvelles élites indigènes et même par ceux qui s’opposent au système colonial, va créer les conditions d'un formidable essor.

Partout, les Français trouvent une administration indigène efficace qui assure la présence royale partout, qui collecte les impôts, rend la justice, administre villages et villes. Les autorités coloniales vont conserver cette administration indigène locale et la faire fonctionner à leur service. Mais cette nombreuse administration indigène doit être formée. Une partie est en relation suivie avec les administrateurs coloniaux: se crée ainsi un besoin de scolarisation en langues locales, mais aussi un besoin limité d'écoles primaires en français. La faiblesse de la population civile française (23700 Français pour 16 millions d'indigènes en 1913, 24482 pour 20 millions en 1921, 34000 pour 22 millions en 1940) oblige aussi les colons et les entreprises coloniales à embaucher des indigènes formés: comptables, secrétaires, infirmiers, cadres moyens, ouvriers qualifiés connaissant bien le français.

Mais c'est Albert Sarraut, partisan d'une mise en valeur des colonies et d'une politique d'association franco-indochinoise qui va accélérer le mouvement en créant un nouveau système d'enseignement franco-vietnamien sans enseignements traditionnels, sans caractères chinois. Il crée

5. Rageau, Christiane. Catalogue du Fonds indochinois: livres imprimés en quôc-ngu, 1922-1954: préface. Paris : Bibliothèque nationale, 1991. p. 5. 
aussi l'université de Hanoi. Il crée donc ainsi de nouveaux lecteurs et de nouveaux auteurs, les futures élites vietnamiennes qui vont favoriser un accroissement considérable de l'édition en français et surtout en vietnamien.

Cette dernière qui dès 1922 est plus importante que l'édition en français, multiplie par trois ou quatre le nombre annuel de titres édités entre 1922 et 1929, la production ensuite se maintient jusqu'en 1942. D’après les statistiques du dépôt légal en 1923: 147 titres édités en vietnamien et 103 en français, en 1929: 643 titres en vietnamien et 233 en français.

Paul Boudet crée la Bibliothèque de Cochinchine à Saigon et la Bibliothèque centrale de l'Indochine à Hanoi. Il organise le dépôt légal spécifique pour l'Indochine; le décret du 31 janvier 1922 institue le dépôt de deux exemplaires de toutes les publications, un pour la Bibliothèque de Hanoi et un autre pour la Bibliothéque nationale de Paris. Le dépôt légal devient effectif pour la plupart des publications. En même temps une liste des imprimés est publiée par la Direction des Archives et des Bibliothèques, 42 fascicules seront édités entre 1922 et 1944.

\section{Description et traitement du fonds indochinois}

Les ouvrages en vietnamien romanisé laissés de côté représentent environ 100 mètres linéaires, 12000 ouvrages environ arrivés surtout entre 1922 et 1940 et dans une moindre mesure entre 1940 et 1954 . Ils sont d'une très grande variété; voici les catégories d'ouvrages et les évaluations de Christiane Rageau:

1. L'apport occidental direct (les traductions d'ouvrages occidentaux) est faible, en nombre limité et majoritairement composé de textes religieux;

2. La vulgarisation de l'apport occidental: manuels scolaires et pratiques, essais sur l'Occident, textes scientifiques et techniques;

3. Le fonds culturel traditionnel: littérature classique vietnamienne et chinoise, textes bouddhistes dont la production double entre 1928 et 1936.

4. La culture populaire nouvelle: nouvelles créations (roman, théâtre, poésie, littérature pour enfants, contes). C'est la catégorie dominante, entre 40 et $60 \%$ du total.

Si on regarde l'évolution de la production de ces quatre ensembles on constate de grandes différences: les deux premiers progressent 
faiblement alors que les œuvres traditionnelles et surtout les nouvelles créations multiplient le nombre de titres.

Si on regarde le nombre de titres le plus important pour ces quatre ensembles, on dessine une chronologie de l'importance des thèmes:

- apport occidental direct: 1925-1927 = 109 titres ;

- vulgarisation: 1928-1930 = 192 titres ;

- fonds culturel traditionnel: 1931-1933 = 237 titres ;

- culture nouvelle : 1934-1936 = 1392 titres.

Les périodiques en vietnamien reçus pendant cette période ont été intégrés dans le fonds général (cotes JO ou Clément) mais il y a dans le fonds indochinois quelques bulletins internes d'associations.

Il y a quelques documents bilingues (par exemple, une conférence sur Huê et ses chansons) et aussi des textes uniquement en français: statuts d'associations indigènes essentiellement, quelques documents de sociétés (Société nouvelle des phosphates du Tonkin ou Société des tuyaux centrifugés de l'Indochine) qui relèveraient davantage des recueils.

Difficiles à traiter en raison de leur langue mais aussi de leur aspect extérieur et de leurs sujets, ces ouvrages ne seront catalogués qu'à partir de la fin des années 1960 sous la responsabilité de Christiane Rageau. Ils seront rassemblés sous une cote unique INDOCH, précédée du format (Fol, 4, 8, 16) et suivie pour la moitié des ouvrages par la mention pièce car il y a de nombreuses brochures. Ils sont recensés dans un catalogue (première édition en 1979, deuxième 1988) puis reproduits sur microfiches de juillet 1986 à juin 1988. Le catalogue est lui-même sur microfiches mais un exemplaire papier est consultable en salle $\mathrm{X}$.

Les 10500 microfiches sont communicables par le système informatique et leur description bibliographique est maintenant accessible dans le catalogue général en ligne.

\section{Un exemple de transfert culturel}

Le catalogage et le microfichage du fonds indochinois auront été l'occasion d'une opération de coopération avec la Bibliothèque nationale de Hanoi, qui a reçu une copie des 12000 microfiches: une partie de ces titres était introuvable dans les bibliothèques vietnamiennes.

Il y a sans doute un lien avec un autre programme de coopération réalisé quelques années auparavant. En 1982, à la suite de la visite du ministre français de l'industrie et de la recherche, un accord de coopération scientifique et technique fut signé entre les gouvernements français 
et vietnamiens. Sur la suggestion de Raymond Aubrac, grand résistant et ami de Ho Chi Minh, il comprendra une étude de repérage de la documentation existant en France au sujet du Vietnam et susceptible de l'aider dans son développement.

La documentation comprend ouvrages, périodiques, thèses, rapports, cartes, archives; quatorze instituts spécialisés, quinze bibliothèques, sept dépôts d'archives vont participer à cette action ambitieuse. La documentation recensée sera présentée par Bertrand Cadiot dans un rapport de quatre gros volumes en 1983.

Pour la Bibliothèque nationale, Nicole Simon et Christiane Rageau rédigent un guide des collections vietnamiennes conservées aux imprimés, aux périodiques et dans les départements spécialisés.

Les Vietnamiens vont ensuite sélectionner des documents qui seront microfichés. Outre de nombreux ouvrages et articles, la totalité des études géologiques préparatoires à la construction du chemin de fer trans-indochinois vont ainsi revenir au Vietnam. 


\section{L'écriture du vietnamien}

\section{Éléments d'histoire à travers le témoignage des collections de la BnF \\ Giang Huong Nguyen}

Dans cet article, nous allons illustrer l'évolution de l'écriture du vietnamien à travers les collections de la Bibliothèque nationale de France. Ce travail vise à démontrer la richesse et la valeur scientifique indiscutables des fonds d'archives et de documents imprimés concernant le Vietnam et leur intérêt majeur pour l'étude de l'histoire de sa langue et de l'histoire de son peuple.

\section{La langue vietnamienne}

Le Vietnam, situé à la jonction de l'Asie orientale et de l'Asie du SudEst, entre la Chine et le monde malais, témoigne des flux de migrations successifs de divers groupes ethniques dès l'époque antique. Les fouilles archéologiques révèlent un brassage entre les groupes de type mélanésien ou indonésien et des éléments mongoliques venus du Nord: les origines du peuple vietnamien sont difficiles à identifier et restent jusqu'à nos jours discutées. Ces incertitudes s'étendent à la langue vietnamienne elle-même.

Dès le $\mathrm{xIX}^{\mathrm{e}}$ siècle, des études sérieuses sont cependant consacrées à celleci; elles sont le fait d'orientalistes occidentaux, notamment du Père Henri Emmanuel Souvignet et d'Henri Maspero. Dans ses Études sur la phonétique historique de la langue annamite (1912), Henri Maspero pointe la contribution à la formation du vietnamien de trois grandes familles linguistiques: le môn-khmer, le thaï et le chinois. Maspero a observé que le vietnamien ne ressemblait au môn-khmer qu'au niveau lexicographique, noté que le chinois différait véritablement du vietnamien au plan syntaxique et insisté 
sur les empreintes importantes du thai ${ }^{1}$. Pour sa part, le père Souvignet avance sa thèse au tout début de son ouvrage Les Origines de la langue annamite (1923): "L'annamite est une langue à fond indo-malais avec superposition de chinois ${ }^{2}$ ", idée matrice qu'il étaye en ciblant des correspondances entre l'annamite et le malais, le khmer, le bahnar, le santali, le nikobarais, le dioi, le thaï, le sanscrit, le chinois et le muong.

Dans les classements contemporains, on considère le vietnamien comme une langue de la branche môn-khmère des langues austro-asiatiques, enrichie au fil des siècles par un nombre important de vocables chinois, avec durant l'époque coloniale, extension au français de cette logique d'emprunt.

Concernant son écriture, le vietnamien bascule successivement dans différents systèmes de transcription:

- L'écriture chinoise traditionnelle que les Vietnamiens appellent le hán;

- Le nôm, l'écriture vietnamienne formée d'idéogrammes empruntant au chinois ;

- Le quốc ngữ, l'écriture vietnamienne moderne en caractères latins.

\section{Présence à la BnF}

Notons d'abord qu'à la Bibliothèque nationale de France, le Vietnam est le premier des pays asiatiques pour lequel a été créé, en 1977, un fonds particulier sous sa cote propre (X011), intégré au fonds général des imprimés. Le vietnamien est la première des langues asiatiques à avoir motivé l'ouverture d'un service autonome au sein du service Asie, département des entrées étrangères; son rôle pionnier s'est encore affirmé en 1988, le vietnamien étant la première langue asiatique à avoir été intégrée dans la base BN-OPALE, avec tous les signes diacritiques nécessaires.

Nous allons donc présenter les différents fonds du vietnamien à travers les jalons que sont les différents systèmes d'écriture de cette langue.

\section{Le fonds en écriture hán}

$\mathrm{Du}^{\mathrm{er}}$ siècle av. J.-C au $\mathrm{x}^{\mathrm{e}}$ siècle apr. J.- $\mathrm{C}^{3}$, le pays Viêt fait partie de l'Empire du Milieu. Durant cette période, les dirigeants chinois cherchent

1. Henri Maspero, Études sur la phonétique historique de la langue annamite: les initiales, Hanoi, F.-H. Schneider, coll. « Bulletin de l'École française d'Extrême-Orient », 1912, p. 118.

2. Henri Emmanuel Souvignet, Les Origines de la langue annamite, 4e édition., Hanoi, Impr. d'Extrême-Orient, coll. "Variétés tonkinoises », 1923, p. 6.

3. Date de l'indépendance gagnée par Ngô Quyền (897-944) qui fonde par la suite le royaume Đại Việt (en 939). 
à intégrer le Vietnam à leur empire en le transformant en une province portant le nom de Giao Chỉ et en usant de multiples moyens d'assimilation politique et culturelle. La langue, les doctrines philosophiques, les mœurs et les coutumes chinoises s'insinuent dans la vie et dans l'esprit des Vietnamiens selon un processus semblable à celui de l'adoption du latin par les Gallo-Romains. C'est que la civilisation de l'Empire chinois est une des grandes civilisations du monde, qui s'est développée très tôt dans l'histoire. Outre le Vietnam, bien d'autres pays en Asie orientale tels la Corée ou le Japon voyaient dans cette culture le symbole de l'humanisme et de l'universalité. Les Vietnamiens apprirent des Chinois, de manière à la fois consentante et rendue obligée par la colonisation, des techniques de culture, des modes de fabrication des outils de travail, une organisation sociale et administrative et, notamment, leur écriture officielle. Ainsi, à la différence de la langue française dont l'utilisation en Indochine résulta directement de la colonisation, l'écriture chinoise fut diffusée au Vietnam de manière certes contrainte mais aussi assez évidente, du fait de la proximité d'un empire influent. Sur une très longue durée, elle s'est imposée dans la création littéraire érudite vietnamienne et est devenue de moins en moins étrangère. Reste que, malgré l'emploi des caractères chinois, les Vietnamiens prononçaient ces derniers de manière tout à fait spécifique (on parle de sino-vietnamien), si bien que Chinois et Vietnamiens ne pouvaient se comprendre à l'oral.

Illustration du statut officiel de l'écriture hán, son emploi monétaire: témoignant d'un règne, d'une dynastie, et de large circulation. Au département des Monnaies et Médailles de la Bibliothèque nationale de France, la collection patrimoniale de monnaies vietnamiennes regroupe quelque deux mille cinq cents pièces qui couvrent la presque totalité du champ historique du Vietnam indépendant ( $\left.x^{\mathrm{e}}-1946\right)$. Il s'agit de la plus importante collection d'Europe et de l'une des toute premières au monde. L'ensemble s'est constitué, depuis le $\mathrm{xIx}^{\mathrm{e}}$ siècle, par acquisition et par don. Citons le don Eugène Gibert, chef d'escadron d'artillerie de marine, en date du 3 juin 1899, qui a enrichi le fonds de la Bibliothèque d'une centaine de monnaies, parmi lesquelles de nombreuses grandes pièces de présentation des Nguyễn (1802-1945); une importante collection de monnaies chinoises, achetée chez Klincksiek, contient une centaine de pièces du Vietnam; et enfin, le don Ciani, fait le 28 février 1929, comprend près de mille cinq cents monnaies. Dans les dons de monnaies chinoises de Ciani, du 20 octobre et du 9 novembre 1928, on trouve également de nombreuses monnaies vietnamiennes classées chinoises par erreur. 
Une politique d'achat sélective, menée depuis 1985 par le département des monnaies a permis, notamment, d'acquérir un rare lingot d'argent du roi Tự Đức ${ }^{4}$ et d'entamer un processsus de de valorisation maximale de cette collection patrimoniale en complétant les séries ${ }^{5}$.

La monnaie vietnamienne est soumise aux mêmes principes que la monnaie chinoise. Des points représentent les étoiles, des cercles le Soleil et des croissants la Lune. Le caractère bảo 寶 " monnaie " figure sur toutes les pièces; sa graphie présente des variantes dont les principales sont les suivantes:

- Le zhen bao, dont l'élément 珍 zhen [précieux] figure à la place de l'élément 尔 ěr [tu] dans la partie supérieure du caractère ;

- Le wang bao, dont l'élément 王 wang [roi] figure à la place de l'élément 尔 dans la partie supérieure du caractère ;

- Et le fou bao 寶, dont l'élément 缶 fou [vase] figure à la place de l'élément 尔 dans la partie supérieure du caractère.

De cette manière, les caractères gravés sur la monnaie nous apportent déjà une idée sur l'état de l'écriture au Vietnam et plus précisément celle du hán à travers chaque époque - le numéraire vietnamien est classé au département des Monnaies selon trois grandes périodes historiques, la conquête et la défense de l'indépendance (939-1428), la dynastie Lê (1428-1788) et la dynastie Nguyễn (1802-1945). En tenant compte de la matière et des caractères qui figurent sur les monnaies, on distingue les monnayages officiels, rebelles et privés attestés, les monnaies incertaines et les copies de monnaies chinoises ou japonaises.

\section{En écriture nôm}

Le nôm est un système d'écriture fondé sur des caractères chinois modifiés et transformés par les Vietnamiens pour transcrire leur langue parlée. Les caractères nôm furent peu à peu utilisés concurremment avec les caractères chinois dans la rédaction des requêtes, des lettres, des actes, des contrats et même de certaines poésies. Cependant, ils n’ont jamais été reconnus comme écriture officielle dans le pays, cette fonction privilégiée étant réservée aux caractères chinois, au hán.

La datation de la naissance du nôm reste jusqu'à nos jours discutée. Certains chercheurs, en s'appuyant sur les légendes populaires, veulent

4. L'empereur Tự Đức (1829-1883), est le 4 e souverain de la dynastie des Nguyễn. Fils de l'empereur Thiệu Trị, il lui succède de 1847 à 1883.

5. Bibliothèque nationale (France), Catalogue des monnaies vietnamiennes, Paris, Bibliothèque nationale, dép. des Monnaies, Médailles et Antiques, 1987, p. 1. 
croire que le nôm a été inventé par Shi Xi 士 焂 (136?-226) [vn. Sĩ Nhiếp], mandarin chinois qui gouverna le district Giao Chỉ, pendant la période de domination chinoise. Cette écriture aurait servi initialement à transcrire les noms propres vietnamiens dépourvus d'équivalents en chinois.

Dans son étude sur les trois systèmes d'écriture du vietnamien, Georges Cordier en renvoie l'apparition à la fin du $\mathrm{xIII}^{\mathrm{e}} /$ commencement du XIv $^{\mathrm{e}}$ siècle, en s'appuyant sur plusieurs sources qui lui semblent plus ou moins fiables. Par exemple, dans les annales dynastiques, le Khâm định Việt sử thông giám cương mục, on peut lire:

Au huitième mois de l'automne de l'an 1282, un crocodile ayant apparu dans le Fleuve Rouge, l'empereur ordonna au ministre de la justice Nguyễn-Thuyên de le chasser à l'aide de charmes. L'opération réussit. L'empereur ayant pris acte de ce que son ministre avait, en ceci, imité le célèbre homme d'état chinois, Hàn Yu, changea le nom de famille de Nguyễn-Thuyên et lui donna celui de Hàn. Il s'appela désormais HànThuyên... Ce Hàn-Thuyên, originaire du huyện de Thanh-lâm, dans la province de Hải-dương, excellait à composer des poésies en langue nationale. Beaucoup de gens l'ayant imité, on appela hàn luật les poésies écrites en chũ̃-nôm ${ }^{6}$.

Un autre livre, le Hải đông chí lược, cité par Georges Cordier, fait état de l'emploi abondant de l'écriture nôm dans la littérature du pays, qui commence avec Hàn Thuyên. L'ouvrage atteste aussi qu'en 1306, circulaient des pamplets en nôm qui raillaient l'union annoncée entre la princesse vietnamienne Huyền Trân et le roi de Champa.

Cordier note également qu'une inscription datée de 1343 a été trouvée gravée sur la montagne Hồ Thanh Sơn, dans la province de Ninh Bình, dans laquelle une vingtaine de caractères nôm sont utilisés pour transcrire des noms de villages et de hameaux.

S'agissant de la constitution des caractères nôm, on peut avoir des emprunts phonétiques, des emprunts sémantiques, des combinaisons d'éléments sémantiques, ou des combinaisons d'un élément sémantique et d'un élément phonétique.

L'emprunt phonétique consiste à utiliser des caractères chinois pour noter un mot vietnamien homophone ou parophone. Exemples:

6. Khâm định Việt sử thông giám cương mục, cité par Georges Cordier, «Les Trois Écritures utilisées en Annam : chữ nho, chữ nôm et chữ quốc-ngữ », Bulletin de la Société d'enseignement mutuel du Tonkin, XV, 1935, p. 117. 
Le Vietnam - Une histoire de transferts culturels

\begin{tabular}{lcccc} 
Caractère & \multicolumn{2}{c}{ Prononciation } & \multicolumn{2}{c}{ Sens } \\
\hline & Sino-vietnamien & nôm & chinois & nôm \\
\hline 卒 & [tốt] & [tốt] & soldat & bon \\
買 & [mãi] & [mấy] & acheter & quelque
\end{tabular}

L'emprunt sémantique consiste à prendre le caractère chinois pour le son seul et de lui affecter le son du mot vietnamien.

Exemple:

\begin{tabular}{ccccc} 
Caractère & \multicolumn{2}{c}{ Prononciation } & \multicolumn{2}{c}{ Sens } \\
\hline & Sino-vietnamien & nôm & chinois & nôm \\
\hline 土 & [thố] & [đất] & \multicolumn{2}{c}{ terre } \\
\end{tabular}

On peut avoir également des formes graphiques purement vietnamiennes, composées par la combinaison d'éléments sémantiques du sino-vietnamien.

Exemple:

\begin{tabular}{ccc} 
Caractère nôm & Sémantique 1 & Sémantique 2 \\
\hline $\begin{array}{c}\text { 胥 trời : ciel } \\
\text { (vietnamien) }\end{array}$ & $\begin{array}{c}\text { 天 thiên: ciel } \\
\text { (sino-vietnamien) }\end{array}$ & $\begin{array}{c}\text { 上 Thượng: haut } \\
\text { (sino-vietnamien) }\end{array}$
\end{tabular}

La combinaison d'un élément sémantique et d'un élément phonétique concerne le caractère complexe comportant deux éléments, sémantique et phonétique: le premier est généralement une clé chinoise; le second, un caractère chinois homophone ou parophone.

Exemples:

\begin{tabular}{lcc} 
Caractère nôm & Sémantique 1 & Sémantique 2 \\
\hline 伵 tớ: serviteur & 人 homme & 四 [tứ] \\
㓠 chém: couper & 刀 couteau & 占 [chiêm]
\end{tabular}

En réalité, loin d'être écriture populaire ou vulgaire, le nôm occupe une place importante dans la société vietnamienne, parallèlement à l'utilisation 
officielle du hán jusqu'à la fin du Xix ${ }^{\mathrm{e}}$ siècle ${ }^{7}$. La littérature en nôm atteint son apogée vers la fin du xviII ${ }^{\mathrm{e}}$ et le début du XIx ${ }^{\mathrm{e}}$ siècle avec des poètes reconnus comme Hồ Xuân Hương (1772-1822), la sous-préfète de Thanh Quan (1805-1848), Đoàn Thị Điểm (1705-1748), et surtout le célèbre poète Nguyễn Du (1766-1820) avec Truyện Kiều (L'Histoire de Kiêu), considéré comme le principal chef-d'œuvre de la littérature vietnamienne.

Les différentes versions de ces œuvres littéraires sont représentées dans le fonds vietnamien des manuscrits orientaux de la Bibliothèque nationale de France. Ce fonds fut créé en 1951-1952, sous la désignation de fonds annamite, par la réunion d'ouvrages dispersés auparavant dans le fonds chinois; de nombreux volumes issus du dépôt légal d'Indochine; d'autres documents provenant d'achats ou de dons enregistrés depuis le début du siècle.

Ce fonds vietnamien est divisé en trois parties: Vietnamien A pour les ouvrages en caractères chinois (A 1 à A 107); Vietnamien B pour les ouvrages en caractères nôm (B 1 à $B$ 134); Vietnamien $C$ pour les papiers d'orientalistes et les textes en quốc ngũo (C 1 à $C 5$ ). Malgré sa volumétrie restreinte de 245 ouvrages, ce fonds contient des éditions xylographiées et des manuscrits de grande valeur. Le Vietnamien A contient des codes de lois, biographies, des pièces administratives, des manuels scolaires et des documents en histoire, en poésie et en théâtre. Le Vietnamien B comprend des ouvrages sur la religion, des manuels, des enquêtes et surtout des romans, des chants et des poésies. Le Vietnamien C est composé de quatre ouvrages seulement, dont une liste due à l'orientaliste Millot, et une histoire de France en quốc ngũ.

Les plus anciens documents, datant du xviII ${ }^{\mathrm{e}}$ siècle, proviennent d'Étienne Fourmont (1690-1746) et du séminaire des missions étrangères: ce sont surtout des vies de saints. S'agissant de ce genre particulier, on note la présence d'une traduction en vietnamien, par l'abbé Masson, de l'Histoire de sainte Élisabeth, reine de Hongrie écrite par Charles-René de Montalembert en 1836; ce dernier en fit don en 1849.

Parmi les principales richesses de ce fonds, on citera les cinq éditions en nôm, et une en hán de Truyện Kiều (L'Histoire de Kiêu) de Nguyễn Du (cf. supra). Par la déclinaison de ses versions en nôm, ce chef-d'œuvre littéraire est aussi d'un apport précieux pour l'étude de l'histoire de l'écriture vietnamienne.

7. Sous la dynastie du roi Hồ Quý Ly (1336-1407) le nôm est même institué comme langue officielle, Hồ Quý Ly fait traduire en nôm quelques classiques confucéens et utilise cette écriture pour introduire le vietnamien à la place du chinois dans les actes administratifs. 
Eu égard à cette étude, le fonds dans son ensemble est d'un notable intérêt. D’une part, il témoigne de la coexistence de trois systèmes d'écriture du vietnamien durant tout le $\mathrm{XIX}^{\mathrm{e}}$ siècle et jusqu'au début du $\mathrm{xx}^{\mathrm{e}}$ siècle. D'autre part, il illustre parfaitement la place cruciale tenue par le nôm dans le passage du système d'écriture emprunté (le hán) à celui propre aux Vietnamiens, le quốc ngũ qu'ils continuent à utiliser jusqu'à nos jours. Notons que les systèmes d'idéogrammes, le hán et le nôm, ne sont guère utilisés au Vietnam à partir du début $\mathrm{du} \mathrm{xx}^{\mathrm{e}}$ siècle. Ils durent céder la place au quốc ngữ [langue nationale], l'écriture romanisée de la langue vietnamienne. La fin de l'époque des idéogrammes est marquée par l'abolition des concours mandarinaux de la Cour impériale en Cochinchine en 1867, au Tonkin en 1916 et en Annam en 1919.

\section{L'écriture quốc ngũ}

Si les deux fonds des monnaies et des manuscrits permettent de se plonger dans l'univers des idéogrammes de la langue vietnamienne, le fonds vietnamien des imprimés témoigne, lui, de manière singulière, du processus de création et de développement de l'écriture vietnamienne moderne, qu'on appelle à l'origine le quốc ngũ.

Le quốc ngũ est une transcription des sons du vietnamien oral en caractères latins agrémentés de signes diacritiques pour rendre compte des tons. Cet outil a été inventé par des missionnaires catholiques portugais, espagnols et français au XvII ${ }^{\mathrm{e}}$ siècle, pour faciliter leur apprentissage de la langue locale. L'acte de naissance du quốc ngứ est le dictionnaire trilingue vietnamien-latin-portugais Dictionarium Annamiticum lusitanum et latinum du père jésuite Alexandre de Rhodes (1591-1660), paru en 1651. Du XvII ${ }^{\mathrm{e}}$ au XIx ${ }^{\mathrm{e}}$ siècle, le quốc ngư est strictement utilisé dans le cadre religieux par les missionnaires européens: employé dans les ouvrages de piété, il est enseigné dans les écoles religieuses aux enfants annamites chrétiens. Le reste de la population vietnamienne, toujours dépendante de la bureaucratie impériale, reste fidèle à l'écriture hán et nôm et à la culture traditionnelle sino-vietnamienne.

Par la suite, le quốc ngữ s'émancipe du cadre chrétien. Il est introduit dans les écoles coloniales franco-indigènes comme un outil de médiation, permettant à la fois de familiariser les Vietnamiens à une approche visuelle de la langue française (que les autorités coloniales envisageaient au début de diffuser largement) et de faciliter l'apprentissage du vietnamien par les Européens.

La graphie du quốc ngũ̃ contient 44 signes qui se combinent: 
27 signes consonantiques

\begin{tabular}{|c|c|c|c|}
\hline Lettre & Prononciation & Lettre & Prononciation \\
\hline $\mathrm{m}$ & $/ \mathrm{m} /$ & ch & /c/ \\
\hline $\mathrm{b}$ & $/ \mathrm{b} /$ & $\operatorname{tr}$ & $/ \mathrm{ts} /$ \\
\hline$p$ & $/ \mathrm{p} /$ & $\mathrm{kh}$ & /kh/ \\
\hline $\mathrm{v}$ & /v/ & đ & $/ \mathrm{d} /$ \\
\hline $\mathrm{ph}$ & $/ f /$ & $\mathrm{t}$ & $/ \mathrm{t} /$ \\
\hline $\mathrm{n}$ & $/ \mathrm{n} /$ & th & $/ \mathrm{t}^{\mathrm{h}} /$ \\
\hline $\mathrm{nh}$ & $/ \mathrm{n} /$ & d & $|z|$ \\
\hline $\mathrm{ng}$ & $/ \mathrm{y} /$ & gi & /j/ \\
\hline ngh & $/ \mathrm{y} /$ & $r$ & $|z|$ \\
\hline g & $/ y /$ & s & $/ \mathrm{s} /$ \\
\hline gh & $/ y /$ & $x$ & /s/ \\
\hline$q(u)$ & $/ \mathrm{w} /$ & I & $/ 1 /$ \\
\hline $\mathrm{k}$ & /k/ & $\mathrm{h}$ & $/ \mathrm{h} /$ \\
\hline c & $/ \mathrm{k} /$ & & \\
\hline
\end{tabular}

12 signes vocaliques

\begin{tabular}{|c|c|c|c|}
\hline Lettre & Prononciation & Lettre & Prononciation \\
\hline i & /i/ & ô & /o/ \\
\hline$y$ & /i/ & $\sigma$ & /ə:/ \\
\hline ê & /e/ & 0 & $/ 2 /$ \\
\hline e & $/ \varepsilon /$ & ă & $/ a /$ \\
\hline u & $/ \mathrm{i} /$ & â & /ə/ \\
\hline $\mathrm{u}$ & $/ \mathrm{u} /$ & $a$ & /a:/ \\
\hline
\end{tabular}

5 signes tonals

\begin{tabular}{lcc} 
Ton & Diacritique & Exemple \\
\hline ngang & nul & bi \\
huyền & $\cdot$ & bì \\
ngã & $\sim$ & bĩ \\
\hline hỏi & $\cdot$ & bí \\
sắc & $\cdot$ & bí \\
nặng & $\cdot$ & bị
\end{tabular}


$\mathrm{Au}$ total, on a 72 signes vocaliques en combinaison avec des signes tonals.

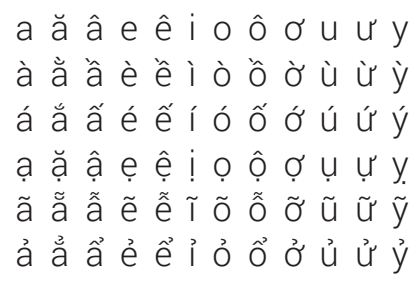

Le quốc ngũ sort des écoles et part à l'assaut de la société avec la parution (de 1865 à 1897), du premier journal en écriture romanisée, le Gia Định báo (Journal de Cochinchine). Initialement destiné à informer des affaires publiques la partie de population qui ignorait tant le chinois que le français, cette publication s'imposa en effet, grâce à son rédacteur en chef, Huỳnh Tịnh Của alias Paulus Của (1834-1907) et son directeur de publication Trương Vĩnh Ký alias Pétrus Ký (1837-1898) comme un instrument actif de vulgarisation du quốc ngữ $\tilde{u}^{8}$ Ce dernier ne tarda pas à devenir un moyen de communication de masse et, progressivement, une langue littéraire. Ainsi portée par l'essor du journalisme en Indochine, la langue vietnamienne trouva chaque jour à s'enrichir, surtout dans les domaines de la philosophie et des sciences, d'apports nouveaux puisés dans les deux cultures: française et chinoise. L'unification de l'écriture s’opéra entre le Nord, le Centre et le Sud, du pays grâce à des échanges de publications et à l'installation dans le Sud des journalistes originaires du Nord et du centre.

À la Bibliothèque nationale de France, la première édition sur microfiches du Catalogue des périodiques vietnamiens remonte à 1993; y contribuèrent Jean-Claude Poitelon et Nguyễn Tất Đắc. Ce catalogue mentionne l'ensemble des documents publiés des origines à 1975 et conservés dans les différents départements de la Bibliothèque. Il s'agit des périodiques publiés au Vietnam, ou rédigés en vietnamien, ou destinés aux Vietnamiens, ou traitant du Vietnam. Les périodiques du Cambodge et du Laos ont été retenus seulement lorsqu'ils entraient, totalement ou en partie, dans l'une de ces dernières catégories. Ce catalogue énumère au total plus de 2350 publications avec près de 500 renvois, et regroupe plus de 2800 fiches. De nos jours, l'alimentation de la bibliothèque en périodiques

8. À la BnF, ce périodique reste incomplet. La collection la plus complète se trouve à la BULAC. 
vietnamiens (126 titres actuellement) se poursuit grâce aux échanges avec la bibliothèque nationale du Vietnam.

Encore aujourd'hui, la richesse et l'importance du fonds vietnamien de la Bibliothèque nationale de France le placent en tête de classement mondial à l'extérieur du Vietnam. Les collections vietnamiennes nous offrent une image fidèle de l'état de la langue et la littérature dans le Vietnam actuel et à travers sa diaspora.

En haut-de-jardin, le fonds vietnamien offre à un large public cinq cents volumes d'ouvrages de référence sur la langue et la culture vietnamienne, des romans contemporains dont certains sont accompagnés de leurs dernières traductions en français, ou à défaut en anglais. Le secteur vietnamien $\mathrm{du}$ fonds récent, Langues vivantes: initiation et pratique, propose des dictionnaires bilingues courants et des outils élémentaires au service des personnes désirant apprendre cette langue.

Concernant la bibliothèque de recherche, le fonds du rez-de-jardin regroupe environ huit cents volumes de romans en langue originale ainsi que d'études linguistiques et littéraires approfondies. De nombreux ouvrages de référence sont aussi proposés: dictionnaires bilingues ou unilingues, généraux et spécialisés, outils biographiques, anthologies; ils permettent aux chercheurs d'avoir une idée plus étayée de l'ensemble de la production littéraire vietnamienne, des origines jusqu'à l'époque contemporaine, dans le pays et dans la diaspora.

Les magasins recèlent plus de cinquante mille titres de monographies dans tous les domaines traditionnellement représentés à la bibliothèque. Les documents arrivent de plusieurs sources - dons, échanges internationaux, acquisitions - et sont répartis entre départements et services selon les disciplines. Ils sont répartis entre quatre catalogues chronologiques:

- Le catalogue du fonds vietnamien 1890-1921 répertorie plus de 1053 titres. Cependant, certains ouvrages, témoins des débuts de la littérature moderne vietnamienne et relevant de la littérature patriotique et de résistance, n’y figurent pas ;

- Le catalogue du fonds indochinois 1922-1954 regroupe de plus de 12000 titres; c'est un fonds patrimonial issu du dépôt légal de l'Indochine à l'époque coloniale ;

- L’inventaire des livres imprimés vietnamiens 1960-1979 réunit 1548 ouvrages, dont la majorité provient de la République démocratique du Vietnam ; 
Le Vietnam - Une histoire de transferts culturels

- Depuis 1980, toutes les entrées sont enregistrées dans le catalogue général en ligne.

$$
* * *
$$

Ainsi, les collections vietnamiennes présentent une grande diversité documentaire qui témoigne de l'évolution de l'écriture vietnamienne en particulier, et de l'histoire du Vietnam, de l'époque monarchique jusqu'au temps présent. Ses catalogues - avec abondance de documents — se sont révélés être des outils indispensables aux chercheurs du monde entier travaillant sur les différentes périodes du Vietnam. Ils leur permettent de s'appuyer sur une identification fine de précieux documents en langue vietnamienne, acquis depuis plus d'un siècle par la Bibliothèque nationale de France. 


\section{0}

\section{Le département des Arts du spectacle}

\section{Focus sur des collections uniques Joëlle Garcia}

La représentation d'un spectacle est par nature éphémère; elle laisse pourtant de multiples traces de son existence. Le département des Arts du spectacle de la Bibliothèque nationale de France conserve l'une des plus riches collections dans le monde où se reflète la fascination des artistes et du public français pour les différentes formes de spectacle venus d'Asie. Cette présentation veut ouvrir quelques pistes vers des sources qui contribuent à l'histoire des relations culturelles entre la France et le Vietnam.

\section{Conserver l'éphémère du spectacle}

Aux origines du département, se trouve la collection privée d'Auguste Rondel (1858-1934), banquier marseillais passionné de théâtre et de spectacle. Étendant sa curiosité bien au-delà des textes dramatiques, il constitue une collection remarquable par son acception très large de la notion de spectacle, et par la diversité des documents rassemblés. À partir de 1892, et jusqu'à sa mort, Rondel rassemble une masse considérable de documents, non seulement autour du théâtre et de l'opéra mais aussi sur les divertissements les plus variés (arts forains, fêtes, expositions, sport...), notamment sur les formes artistiques émergentes que sont alors le cinéma et le théâtre radiophonique. Rondel collecte des livres, des brochures, mais aussi des articles de presse, et des images de toute nature. Il recueille également les archives de ses amis praticiens, et des lieux de spectacle. La valeur de cette bibliothèque privée, ouverte au public, est 
rapidement reconnue. En 1920, Auguste Rondel en fait don à l'État. Après quelques péripéties, la collection est abritée à la bibliothèque de l'Arsenal. À la mort de Rondel, le travail de collecte est poursuivi dans le même esprit. Des séries documentaires sont constituées par type de documents. Sont régulièrement accueillis de nombreux fonds d'archives privées concernant l'activité d'auteurs, de metteurs en scène, de cinéastes, de réalisateurs, de théâtres et de compagnies, d'acteurs, de chanteurs, de danseurs, de mimes, etc. En 1976, sous l'impulsion d'André Veinstein, homme de théâtre qui s'est consacré à l'étude et l'enseignement des arts du spectacle, les collections théâtrales intégrées à la Bibliothèque nationale forment un département à part entière.

Aujourd'hui le département des Arts du spectacle conserve, donne à consulter et enrichit quelque quatre millions de documents, sur tous les domaines du spectacle vivant (théâtre, théâtre lyrique, danse, mime, cirque, marionnettes, chanson, cabaret, music-hall, fêtes, feux d'artifices) mais aussi le cinéma jusqu'en 1945 et la radiotélévision jusqu'à la fin des années 1960. Dans ses collections, se trouvent aussi bien des livres, des périodiques, des manuscrits, des correspondances, des tapuscrits, que des archives privées, des archives administratives, des maquettes de décors et costumes, des dessins, des estampes, des photographies, des affiches, des programmes, des coupures de presse, des archives sonores et audiovisuelles, des costumes, des accessoires, des marionnettes, des peintures, des sculptures, et autres œuvres d'art. Cette diversité témoigne de l'ensemble du processus complexe de l'élaboration d'un spectacle, de la conception d'une œuvre (documentation préparatoire, élaboration du texte, travail sur le plateau), de sa représentation (conduites et plans techniques, maquettes, costumes, accessoires, éléments de décor, musique de scène, photographies, affiches, programme), de sa réception auprès du public (presse, lettres de spectateurs, études).

\section{Les spectacles en Indochine dans la collection Rondel}

Les documents concernant l'Indochine sont principalement classés dans une sous-section consacrée au théâtre des provinces et des colonies de la section créée pour le théâtre français (Rf) mais aussi dans la section consacrée au théâtre étranger (Re) qui rassemble les textes dramatiques et littéraires en langue originale ou traduits, des documents sur l'histoire et la vie théâtrale de chaque pays, les activités des compagnies et les carrières des acteurs. Se trouvent également des documents dans les séries 
consacrées au cinéma (Rk), aux arts des fêtes, feux d'artifices, spectacles de cour ( $\mathrm{Ra}$ ) et dans les séries iconographiques (ICO).

Les éditions de textes dramatiques collectées par Auguste Rondel reflètent la vie théâtrale de l'Indochine entre 1900 et 1930. Au fil des références, se déroulent le répertoire français diffusé dans le pays par des éditeurs à Saigon ou Hanoi, les comédies, opérettes et revues données au théâtre municipal de Saigon inauguré en 1900, de Hanoi en 1911 et de Haiphong en 1912, spectacles représentés par des troupes venues spécialement de France pour la saison théâtrale ou par des troupes en tournée en Asie. La progression du théâtre classique se manifeste avec la traduction en vietnamien de Molière, Corneille et Racine; y apparaît un théâtre vietnamien. Si ces éditions se trouvent également, pour la plupart, dans les collections entrées par dépôt légal à la Bibliothèque nationale, quelques volumes ou pièces ne sont conservés que dans la collection Rondel.

L'intérêt de la collection réside dans les recueils constitués de programmes et de coupures de presse. En effet, lorsqu'Auguste Rondel commence sa collection, la critique dramatique, véritable genre littéraire, l'actualité des spectacles et ses échos mondains prennent une place importante dans les quotidiens et se révèlent une source de premier plan. Le découpage quotidien de la presse, la constitution méthodique de ces coupures en recueils permet de constituer un ensemble documentaire exceptionnel. Sont ainsi conservés des recueils factices d'article de presse et de programmes concernant le théâtre français en Indochine entre 1904 et 1931, le théâtre à Hanoi entre 1926 et 1928 (direction Bourrin), le théâtre à Saigon entre 1899 et 1930, le théâtre indochinois, le théâtre annamite, le cinéma en Indochine...

Dans les décennies où Rondel décide des principes de constitution de sa collection, la photographie se développe dans la presse, les programmes; le public s'arrache les portraits-cartes photographiques des grandes vedettes de l'époque. La constitution de recueils factices d'images de presse, de dessins, de photographies et de cartes postales aux côtés de dossiers de presse fait alors particulièrement sens. Ces lots d'images documentant les spectacles, les artistes, l'architecture des salles, comme, par exemple, sur l'architecture du théâtre de Saigon ${ }^{9}$, présentent des ensembles exceptionnels.

Ces documents trouvent parfois un écho dans d'autres collections entrées au département des arts du spectacle. Ainsi, un film sur les sports indochinois tourné en 1911 se trouve documenté par une coupure de

9. BnF, ASP, 4-ICO-ARC-372. 
presse dans la collection Rondel ${ }^{10}$ et par un synopsis ${ }^{11}$ conservé dans une collection de synopsis de films distribués par la firme Pathé entre 1909 et 1917. Entrés à la Bibliothèque nationale par le dépôt légal des imprimés, et versés au département des Arts du spectacle à la fin des années 1970, ces feuillets portent au recto un résumé de l'histoire et parfois de la distribution; au verso, sont collées une ou plusieurs bande(s) de photogrammes extraits du film. Le synopsis du film Sports en Indochine nous apprend ainsi que le film faisait 135 mètres de pellicule, montrait des courses de pirogues, concours de natation, lutte de champions chinois, luttes de nains, et nous en présente quelques rares photogrammes.

\section{Des relations culturelles visibles dans les fonds d'archives}

Plusieurs fonds d'archives de personnalités du théâtre et du cinéma contiennent des correspondances qui témoignent aussi bien de l'organisation des spectacles en Indochine pendant la période coloniale, que des échanges culturels entre la France et l'Indochine puis le Vietnam. Par exemple, dans le fonds du comédien, metteur en scène, directeur de théâtre, réalisateur et critique français André Antoine ${ }^{12}$ (1858-1943), se trouvent quelques lettres de G. Cotty sur la saison 1924-1925 de Saigon ou un dossier de candidature de Claude Bourrin à la direction du théâtre municipal de Saigon. Dans le fonds de l'écrivain, acteur, auteur dramatique, scénariste et réalisateur de cinéma Marcel Achard (1899-1974) ${ }^{13}$, une abondante correspondance avec la famille du docteur Nguyen Manh Don $^{14}$, ami proche du couple Achard, témoigne de l'histoire et du quotidien d'une famille vietnamienne de 1943 à 1978.

Signalons tout particulièrement le fonds de l'écrivain vietnamien Phạm Văn Ký (1916-1992) ${ }^{15}$. Écrivant pour plusieurs revues à Saigon puis à Huế, Phạm Văn Ký a publié deux premiers romans en vietnamien, puis a poursuivi son œuvre en français. Il a reçu, en 1936, le premier prix de poésie aux Jeux floraux d'Indochine pour son recueil de poèmes en

\footnotetext{
10. BnF, ASP, 8-RK-10583.

11. BnF, ASP, 4-COL-4 (5322).

12. BnF, ASP, COL-113.

13. BnF, ASP, COL-101.

14. Connu pour avoir introduit l'acupuncture en France, Nguyen Manh Don a également été acteur de cinéma (il a notamment joué dans le film de Georg-Wilhelm Pabst Le Drame de Shanghai).

15. BnF, ASP, COL-25.
} 
français Une voix sur la voie. Venu à Paris en 1938 pour poursuivre ses études, il devint professeur de français, puis rédacteur à la préfecture de la Seine. À la Libération, il entra à la radiodiffusion française où il devint par la suite responsable du service français des émissions radiophoniques destinées à l'Indochine. Il a écrit des pièces radiophoniques telles que $L a$ Muraille de Chine, Gandhi ou Attila Roi (prix de la Mise en ondes en 1954). Il a collaboré à diverses revues littéraires et publié plusieurs romans dont Frère de sang, Celui qui régnera, Perdre la demeure (grand prix du roman de l'Académie française en 1961), des recueils de poèmes, dont Fleur de jade, l'argument du ballet Danse avant l'aube, une pièce de théâtre, Le Rideau de pluie, jouée en 1974. Ce fonds contient principalement les manuscrits de ses romans, œuvres poétiques et dramatiques, essais, scénarios, contes et nouvelles. Plusieurs de ces textes sont inédits. Le fonds contient également des archives personnelles, des photographies de la correspondance, ainsi que des articles écrits sur ses œuvres. Comme l'a montré Giang Huong Nguyen ${ }^{16}$, Phạm Văn Ký évoque dans plusieurs de ses textes l'identité vietnamienne dans ses rapports avec la civilisation française.

Plusieurs fonds témoignent également des spectacles créés en France qui ont été inspirés par l'histoire vietnamienne ou sa culture. Prenons l'exemple du Théâtre du Soleil, dont les créations collectives sous la direction d'Ariane Mnouchkine se sont souvent inspirées des traditions orientales. Dans L'Histoire terrible mais inachevée de Norodom Sihanouk, roi du Cambodge, spectacle créé par le Théâtre du Soleil en 1985, l'auteur Hélène Cixous présente une vision de l'histoire du Cambodge entre 1955 et 1979. Sous la forme d'un récit épique, sont mis en scène des événements récents de l'histoire cambodgienne, dont le Vietnam est partie prenante. En dialogue avec les archives d’Hélène Cixous conservées au département des Manuscrits de la Bibliothèque nationale de France, les archives du fonds du Théâtre du Soleil ${ }^{17}$ documentent toutes les étapes de la création et de la vie de ce spectacle, depuis la documentation constituée par l'auteur et la troupe pour nourrir le travail d'écriture et de plateau jusqu'aux lettres de spectateurs. Un autre spectacle créé en 1999, Tambours sur la digue, fable politique et philosophique imaginée par Hélène Cixous, est fortement imprégné par les formes orientales de la marionnette. Les habitants d'une Chine mythique et archaïque sont confrontés à la menace d'une

16. Giang Huong Nguyen, Phạm Văn Ký: une rencontre de l'Orient et de l'Occident. Le théâtre français en Indochine, oct. 2013, Marseille, France. < hal-01059875 >

17. BnF, ASP, COL-153. 
inondation qui risque d'emporter les digues protectrices de la cité. Pour ce spectacle, la metteur en scène Ariane Mnouchkine a choisi une forme inspirée du nô et du bunraku japonais: des acteurs masqués campent des poupées vivantes, actionnées par des acteurs manipulateurs. Dans la scène finale, les personnages sont figurés par des marionnettes miniatures manipulées sur l'eau qui a envahi le plateau. La technique des marionnettes sur eau du Vietnam a en effet nourri le travail sur ce spectacle. Dans le fonds du théâtre du Soleil sont conservées ces émouvantes marionnettes de tissu, de fil de fer et de polystyrène, qui nous rappellent la fragilité des traces du spectacle.

$$
* * *
$$

Ce panorama rapide n'évoque pas toutes les collections et tous les fonds du département des Arts du spectacle qui contiennent des documents utiles à l'étude des relations culturelles entre la France et le Vietnam mais montrent la richesse et la variété des sources disponibles. Une grande partie de ces collections et fonds est décrite ou en cours de description dans les catalogues de la $\mathrm{BnF}^{18}$. Des documents restent à découvrir dans des ensembles qui, même en cours d'inventaire, sont consultables dans la salle de lecture du département des Arts du spectacle. Ce patrimoine méconnu invite à diversifier les sources et les approches pour approfondir notre connaissance de l'histoire des spectacles comme des relations culturelles et des influences croisées entre les deux pays.

18. Pour la collection Rondel, la collection de monographies et de périodiques, la collection de dessins, de maquettes, de documents iconographiques, d'affiches, les archives photographiques, les dossiers de presse constitués à partir de la $2^{\mathrm{e}}$ moitié du XX $\mathrm{X}^{\mathrm{e}}$ siècle, consulter le catalogue général (catalogue. bnf.fr); pour les manuscrits et les fonds d'archives, consulter le catalogue Archives et manuscrits (archivesetmanuscrits. bnf.fr). 


\title{
21
}

\section{Ressources photographiques concernant le Vietnam}

\author{
Les plaques de verre \\ de la Société de géographie \\ Olivier Loiseaux
}

La Société de géographie a été fondée en 1821 avec pour but de concourir aux progrès de la géographie, d'encourager les études et les découvertes géographiques et de faire entreprendre des voyages dans des contrées inconnues.

Une bibliothèque est prévue dès l'origine mais sans budget d'acquisitions. Elle s'enrichit essentiellement par don et échanges de publications. Sont conservés des récits de voyage, des revues de géographie, des cartes, des atlas et dans le dernier quart du XIX ${ }^{\mathrm{e}}$ siècle des photographies. Cette bibliothèque est installée en 1878 dans l'hôtel du 184 boulevard SaintGermain que la Société de géographie fait construire

La collection photographique nait à cette période sous l'impulsion de James Jackson, le bibliothécaire de la Société de géographie qui est lui-même photographe, soutenu dans cette entreprise par le secrétaire général de la société, Charles Maunoir.

Jackson sollicite les dons par une circulaire envoyée à tous les membres. Plus de 15000 photographies papier seront reçues pendant son mandat à la tête de la bibliothèque.

Parallèlement, la Société de géographie organise depuis 1875 des conférences illustrées par des projections photographiques. Elle a à la fin $\mathrm{du} \mathrm{XIX}^{\mathrm{e}}$ siècle son projectionniste attitré en la personne d'Alfred Molteni, issu d'une longue famille de fabricant d'appareils d'optique. Le principe est de faire réaliser pour chaque conférence une série de vues positives 
sur verre (de format 8 x $10 \mathrm{~cm}$ ) qui seront projetées à l'aide d'un appareil utilisant la lumière oxhydrique. À l'issue de la conférence ces positifs de projection sont donnés à la Société de géographie.

Ce fonds photographique qui s'enrichit depuis la fin du XIX ${ }^{\mathrm{e}}$ siècle comprend aujourd'hui 100000 tirages papier et 40000 plaques de verre. Il est en dépôt à la Bibliothèque nationale de France sous la responsabilité du département des Cartes et Plans.

La collection de plaques de verre (positifs de projection mais aussi plaques négatives et stéréoscopiques) est en cours d'inventaire et de numérisation. Cette numérisation est visible dans Gallica, la bibliothèque numérique de la BnF et pour les documents sous droits d'auteur dans les salles de lecture de la BnF via Gallica intra-muros.

Plus de 1500 plaques de verre concernant l'Indochine sont déjà inventoriées dont les deux tiers se rapportent au Vietnam. C'est sur cette partie de la collection que portera cet article en deux parties: la collection des positifs de projection et le fonds Firmin-André Salles constitué de plaques négatives et positives.

\section{Les projections de vues sur verre}

Une première conférence sur l'Annam et la Cochinchine est donnée en 1879 par Tran Nguyen Hanh, répétiteur à la chaire de vietnamien de l'école des langues orientales et membre de la Société de géographie commerciale de Paris. Cette conférence est illustrée mais par des clichés sur verre établis d'après des photographies d’Émile Gsell.

C'est véritablement à partir de 1885-1890 que l'Indochine s'invite dans les thèmes des conférenciers à la Société de géographie.

Pierre Mirande, médecin de la Marine, donne une conférence le $1^{\text {er }}$ décembre 1893 sur les Ba-Bé, une région montagneuse du Nord du Tonkin qu'il a visitée quelques mois auparavant. Il s'est rendu aux grottes de Pung, une montagne traversée par un fleuve, le Song Gam formant un tunnel long de 350 mètres. Mirande explique la complexité de cette curiosité géographique à l'aide de croquis cartographiques sur verre qu'il fait réaliser pour l'occasion par le cartographe de la Société de géographie, Jules Hansen. Les parois de ce tunnel donnent accès à deux immenses grottes occupées par plusieurs villages (fig. 1). Mirande visite les différentes communautés de cette région et photographie les habitants dans leurs occupations quotidiennes.

Le lieutenant de vaisseau Marie Léon Escande est un officier de marine spécialisé dans l'hydrographie et la géodésie. Il participe à 
plusieurs opérations pendant la guerre du Tonkin et est remarqué pour ses travaux hydrographiques sur le fleuve Rouge (fig. 2). Au cours de ses campagnes, il effectue des levés du fleuve et procède à de nombreuses études hydrographiques tant la navigabilité du fleuve, voie de pénétration et axe stratégique vers la Chine, est un enjeu important. À son retour en France, il donne à la Société de géographie, le 15 février 1895, une conférence illustrée par des clichés photographiques qu'il a réalisés en complément de ses études.

Gabrielle Vassal est une des premières femmes à donner une conférence à la Société de Géographie; elle intervient à la tribune le 17 mars 1911. Après trois ans passés en Annam, aux côtés de son mari nommé sous-directeur de l'Institut Pasteur de Nhatrang, elle livre ses souvenirs et laisse une centaine de clichés sur verre à la Société de géographie.

Durant cette période, elle a eu l'occasion de se rendre dans les villages moïs sur le plateau du Lang Bian où est projeté l'installation d'un sanatorium. Elle relate plusieurs épisodes cynégétiques et montre son goût pour la chasse aux grands fauves.

Léon Busy, intendant militaire en Indochine et au Tonkin entre 1898 et 1917 fut un des opérateurs d'Albert Kahn pour son projet des archives de la planète. Il a laissé à la Société de géographie, probablement pour une projection, une cinquantaine de clichés de la région de Lang Son, dans le nord du Tonkin, pris en 1920. Busy s'intéresse avant tout aux populations de cette région proche de la frontière chinoise et fait preuve d'une grande maîtrise dans la technique photographique à travers de saisissants portraits et scènes de groupes.

\section{La collection Firmin-André Salles}

C’est en 1921 que Firmin-André Salles, membre de la Société de géographie, a donné sa collection de plusieurs milliers de photographies sur papier et plaque de verre.

Pour la seule Indochine, cette collection est forte de plus d'un millier de plaques négatives et de positifs de projection s'étendant sur deux décennies (1885-1904). L’inventaire des positifs de projection est achevé. Les plaques négatives ( 9 × $12 \mathrm{~cm}$; 13 x $18 \mathrm{~cm}$ ou 18 x $24 \mathrm{~cm})$ sont en cours d'inventaire et de numérisation.

Firmin-André Salles est né à Tarbes en 1860 mais débute sa carrière comme commissaire de la marine, attaché au port de Brest. En $1884 \mathrm{Il}$ prend part aux opérations conduites par l'amiral Courbet qui marquent le début de la guerre du Tonkin. Il pratique déjà la photographie, légende 
et date précisément ses clichés. Les toutes premières photographies que nous conservons sont réalisées dans ce cadre: une série de vues de la baie de Ha Long prises les les 5 et 6 juillet 1885 (fig. 3).

En 1894, il réussit le concours d'inspecteur des colonies et retourne à ce titre en Indochine où il va rester quatre ans (1895-1898). Il assiste l'inspecteur général Verrier dans son travail. Son travail photographique est considérable et couvre tous les domaines.

Il photographie les paysages, les ouvrages d'art, forts et ponts qui jalonnent la route Mandarine. Il s'intéresse à l'architecture: les monuments célèbres (tours Cham) se retrouvent dans ses clichés. Il porte un même intérêt aux événements de la vie coloniale et aux scènes de la vie quotidienne: travaux des champs, petits métiers des rues des grandes villes, processions et fêtes. D’une compréhension moins évidente, une série de portraits de sous-officiers des troupes indigènes où se mélangent préoccupations ethniques et anthropologiques, ou encore une saisissante galerie de portraits de détenus du bagne de Poulo Condor.

Hanoi occupe une place particulière dans sa production photographique à travers deux séries de scènes: les principaux sites et monuments religieux aux abords du lac Hoan Kiem (fig. 4) et les rues commerçantes de la ville marchande (fig. 5).

Par l'ampleur de son travail Firmin-André Salles peut être considéré comme l'un des pionniers du reportage photographique.

\section{Pour consulter les images:}

- Bibliothèque numérique Gallica: http://gallica.bnf.fr/Search?ArianeWireIndex=index\&p=1\&lang=FR \&f_typedoc=images\&q=salles+Vietnam

- Exposition virtuelle "Visions de Hanoi »: http://expositions.bnf.fr/socgeo/vietnam/01.htm 


\section{Table des matières}

Les auteurs ....................................................... 3

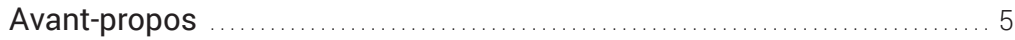

\section{PREMIÈRE PARTIE}

Mise en perspective théorique et historique ............................ 13

1. Les transferts culturels franco-vietnamiens (Michel Espagne) ....... 15

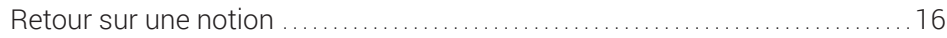

Transferts en contexte colonial ....................................... 18

Le Vietnam ou la perpétuité du transfert................................ 19

Une architecture urbaine .......................................... 22

Dalat, ville-transfert ............................................ 23

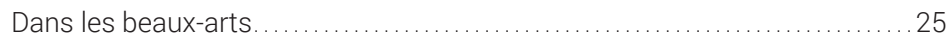

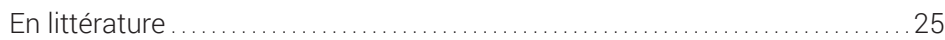

Dynamiques de la langue ........................................ 29

2. Un laboratoire pour l'étude des transferts (Philippe Papin) ............ 33

Maillon d'empire et croisée des chemins ............................. 33

L'emprunt, signe d'activité politique et sociale ............................... 35

Usages, contextes et diversité ...................................... 37

L'adaptation est-elle systématique ? . .................................. 38

La reprise et la réplique........................................ 40

Vertu de la duplication. .............................................. 42

Rupture, déconnexion, placage de références nouvelles ...............43

\section{DEUXIÈME PARTIE}

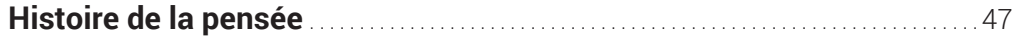

3. Vents d'Est, vents d'Ouest (Phuong Ngoc Nguyen) ................. 49

Début du $x x^{e}$ siècle : crise et ouverture ............................... 50

Acteurs, moments, lieux, moyens de communication .................... 54

Quelles Lumières ?................................................... 61

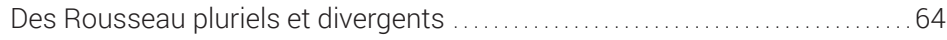


4. La catholicité vietnamienne (Pierre Brocheux) …......................69

L'ouverture de l'Asie à l'évangélisation....................................69

Implantation au Đại Việt. ................................................. 70

Des persécutions à l'hypothèque coloniale ............................. 71

La décolonisation de la catholicité vietnamienne........................ 74

Des décennies 1930 et 1940 décisives ................................ 77

5. Un bouddhisme vietnamien de France? (Jérôme Gidoin) ..............81

Un arrière-plan politique............................................. 82

L'ÉBVU en France ................................................... 85

L'activisme bouddhique en terre d'exil................................ 87

Un essor via la prise en charge des hommages aux défunts .............. 88

Assimilation de l'identité bouddhique à l'identité culturelle ................90

La pagode, un symbole de la vietnamité en France ....................... 92

\section{TROISIÈME PARTIE}

Construction des savoirs ............................................... 95

6. L'économie hybride du post-Champa (Andrew Hardy) .................. 97

Le modèle économique post-Champa ................................ 100

Les places d'échanges ............................................. 103

Les relais ......................................................... 110

7. Des orientalistes en Orient (Pierre-Yves Manguin) ................... 115

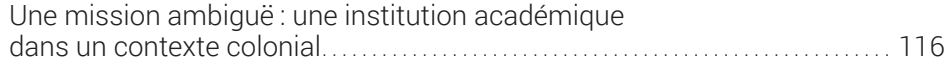

Le legs de l'EFEO au Vietnam........................................ 121

La dette de l'EFEO : les lettrés de Hanoi ..................................... 128

8. Pratiquer la médecine enseignée à la française

(Laurence Monnais) ............................................. 133

Un bref portrait quantitatif et qualitatif............................... 134

L'école de médecine de Hanoi et ses meilleurs étudiants ................ 135

Expatriation, expertise et hiérarchies.............................. 137

De la sphère publique à la sphère privée .............................. 139

Un vent de liberté lucratif .......................................... 140

À propos de l'offre et de la demande ....................................... 142

Défenseurs d'un pluralisme médical ?.................................... 143

9. L'Université indochinoise et l'œuvre culturelle de la France au Vietnam (Tuân Hoang Van) ...................................... 151

La naissance et le développement de l'enseignement supérieur au Vietnam152

Les objectifs de l'enseignement supérieur au Vietnam ..................... 157

L'accueil par les Vietnamiens de ce nouveau système d'enseignement. .. 159

L'influence de l'enseignement supérieur sur les Vietnamiens.............. 161

L'influence de la culture française sur celle du Vietnam ................... 164

Une œuvre de portée limitée ...................................... 166 
10. Sel, soufre et mercure (Thuy Phuong Nguyen) ..................... 169

Pour une histoire des élèves............................................. 169

Un quotidien multiculturel .......................................... 171

Entre deux cultures ................................................. 179

La base initiale d'une vie ............................................ 182

11. L'ère des tempêtes (Alain Ruscio) .................................. 187

Le général Navarre : un nouveau sauveur ? ............................. 187

L'assaut......................................................... 188

La première vague ............................................... 190

Nos héros face à la ruée jaune ....................................... 193

Une intervention américaine directe ? .............................. 196

La chute ................................................... 198

La fin d'une guerre .............................................. 200

Épilogue ........................................................... 201

\section{QUATRIÈME PARTIE}

\section{Arts et littérature.}

12. Deux mondes artistiques, une rencontre (Nadine André-Pallois). . 205

L'Orient des peintres français. ........................................ 206

Premiers regards, un état des lieux ............................... 207

Rendus d'humanité ............................................... 207

L'École des beaux-arts de l'Indochine ................................ 212

Les fruits de la rencontre............................................ 216

Bibliographie ........................................................ 219

13. Le sujet lyrique dans la poésie de Han Mac Tu

(Hoai Huong Aubert-Nguyen) ...................................... 221

Le sujet lyrique dans la poésie romantique française.................... 222

La structure de l'énonciation dans la langue vietnamienne ............... 223

Chez Han Mac Tu : le sujet lyrique de la période classique................ 224

Affirmation d'un sujet lyrique pluriel .................................. 225

Le sujet lyrique et l'altérité .......................................... 230

\section{Légendes des terres sereines de Pham Duy Khiêm}

(Thanh Vân Tôn Thât) ............................................... 235

Parcours d'un homme et premiers transferts ........................ 236

Esquisse de classement et de typologie............................ 238

Enquête littéraire du collectionneur et quête identitaire .................. 242

15. Entrecroisements littéraires (Julie Assier) ....................... 249

Itinéraire d'une francophile vietnamienne .......................... 250

Ancrage occidental, traces extrême-orientales .......................... 252

L'histoire du Vietnam à la lumière des mythes gréco-romains............ 256

16. Une nouvelle ère du roman? (Cam Thi Doan) ..................... 261

Évolution d'un genre ............................................. 261

Quête et conquête ................................................. 265 
Le Vietnam - Une histoire de transferts culturels

17. Mémoire poétique et identité historique (Veronica Ntoumos)..... 273

Contexte de l'œuvre................................................ 274

Fiction et histoire : mode de représentation polyphonique ............. 275

Douloureuse réinvention ......................................... 276

Transferts culturels, sociaux et graphiques ......................... 277

CINQUIÈME PARTIE

Les collections vietnamiennes de la Bibliothèque nationale

de France

18. Le fonds indochinois de la Bibliothèque nationale de France

(Denis Gazquez) .................................................... 283

Les fonds sur le Vietnam colonial dans les départements spécialisés. ... 283

Les fonds imprimés sur le Vietnam colonial dans les départements

thématiques de Tolbiac .............................................. 285

Le fonds indochinois : historique d'un fonds ....................... 286

Description et traitement du fonds indochinois.......................... 288

Un exemple de transfert culturel ................................... 289

19. L'écriture du vietnamien (Giang Huong Nguyen) .................... 291

La langue vietnamienne ............................................... 291

Présence à la BnF ................................................. 292

L'écriture quốc ngữ .................................................... 298

20. Le département des Arts du spectacle (Joëlle Garcia) ............. 303

Conserver l'éphémère du spectacle ....................................... 303

Les spectacles en Indochine dans la collection Rondel .................. 304

Des relations culturelles visibles dans les fonds d'archives................ 306

21. Ressources photographiques sur le Vietnam (Olivier Loiseaux)... 309

Les projections de vues sur verre ..................................... 310

La collection Firmin-André Salles .................................. 311 


\section{Déjà parus aux éditions DEMOPOLIS}

Brossat, Alain

Abécédaire Foucault

Boltanski, Luc

Rendre la réalité inacceptable

Bourdieu, Pierre - Boltanski, Luc

La Production de l'idéologie

dominante

Césaire, Aimé - Malcolm X

Black revolution

Clover, Charles

Surpêche

Da Lage, Olivier (sous la dir. de)

Qatar: les nouveaux maîtres du jeu

Denord, François

Néo-libéralisme, version française

Duclert, Vincent

Occupy Gezi

Durpaire, François -

Richomme, Olivier

L'Amérique de Barack Obama

Obama face à la crise

Gaulard, Mylène

Karl Max à Pékin - Les racines de

la crise en Chine capitaliste

Garo, Isabelle

Foucault, Deleuze, Althusser et Marx

Hobsbawm, Éric

Marx et I'histoire

Hroub, Khaled

Le Hamas

Jaurès, Jean

Le socialisme et la Révolution

française

Jennar, Raoul Marc Khieu

Samphan \& les Khmers rouges

Khaldy, Eddy - Fitoussi, Muriel
Main basse sur l'École publique

La République contre son école

Kalfon, Pierre

Chroniques chiliennes

Kamata, Satoshi,

Toyota: I'usine du désespoir

Mamdani, Mahmoud

La CIA et la fabrique

du terrorisme islamique

Labat, Séverine

Les islamistes tunisiens - entre

l'État et la mosquée

Latour, Bruno - Lippman, Walter

Le public fantôme

Lénine

Petit manuel pour rompre avec le capitalisme

Lénine

1914, repenser le nationalisme et

la guerre

Marx, Karl

Qu'est-ce que le capitalisme?

Les Crises du capitalisme

Le Capital financier

Mordillat, Gérard -

Prieur, Jérôme

De la crucifixion considérée

comme un accident du travail

Nsar, Vali

Le renouveau chiite

Pivert, Marceau

L'Église et l'École

Prochasson, Christophe

L'Empire des émotions:

les historiens dans la mêlée

Rebérioux, Madeleine

Vive la République 
Rodinson, Maxime

Islam et capitalisme

Sassen, Saskia

Critique de l'État

Saurin, Patrick

Les prêts toxiques: une affaire d'État

Shah, Sonia

Cobayes humains: le grand secret des essais pharmaceutiques

Uchitelle, Louis

Le salarié jetable

Wallerstein, Immanuel

L'Universalisme européen:

de la colonisation au droit

d'ingérence

Wilkinson, Richard

L'égalité, c'est la santé

\section{Collection « QUAERO »}

Cassin, Barbara et Wosny, Danièle (dir.)

Les intraduisibles du patrimoine en Afrique subsaharienne

Dawod, Hosham (dir.)

La constante «Tribu », variations

arabo-musulmanes

Ehrenfreund, Christian et

Schreiber, Jean-Philippe (dir.)

Les marranismes. De la religiosité

cachée à la société ouverte

Fontaine, Alexandre

Aux heures suisses de l'école

républicaine

Ghasarian, Christian

Rapa. île du bout du monde, île dans le monde

Niveleau, Charles-Édouard (dir.)

Vers une philosophie scientifique Le programme de Brentano

Rabault-Feuerhahn, Pascale (dir.)

Théorie intercontinentales

- Voyages du comparatisme postcolonial

Salamagne, Michèle- $\mathrm{H}$. et Thominet, Patrick (dir.),

Accompagner. Trente ans de soins palliatifs en France 
«Second tirage»

Achevé d'imprimer en France en 2016 dans les ateliers de Dupli-print à Domont (95) $\mathrm{N}^{\circ}$ d'impression : 2016010105 


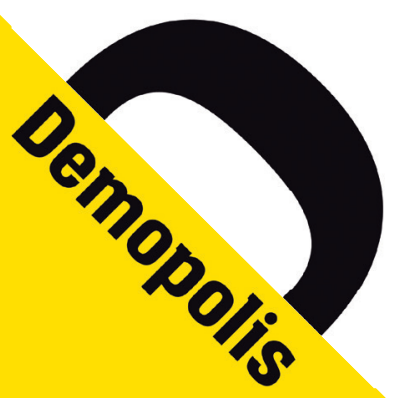

Loin de se résumer à un champ de bataille ou à un espace colonial dont l'émancipation a déclenché tous les mouvements de libération ultérieurs, le Vietnam a été l'un des lieux les plus frappants de rencontres entre les peuples et les cultures dans l'histoire humaine, un creuset de civilisations par rapport auquel les melting pots les plus souvent invoqués se résument à des

équations beaucoup plus simples. Il y a certainement un moment vietnamien de l'histoire globale, les transferts culturels qui ont formé le pays donnant un écho universel aux cataclysmes qui se sont abattus sur lui. L'histoire ancienne du pays est déjà une histoire d'imbrications entre les cultures, chinoise et indienne. L'histoire moderne l'est davantage encore. De la littérature aux beaux-arts en passant par les phénomènes religieux, le système éducatif ou la constitution des savoirs, le temps est venu d'observer le dense réseau d'interactions qui constituent le Vietnam et le relient au monde.

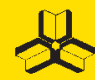

SODIS : 7235500

ISBN : 978-2-35457-077-4

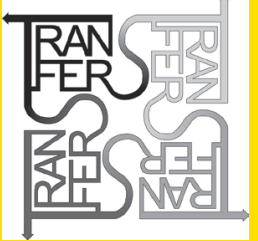

Ce livre a été conçu par Hoai Huong Aubert-Nguyen, enseignante de lettres et écrivain, et par Michel Espagne, historien des transferts culturels. Il rassemble vingt et un auteurs issus de disciplines très différentes : spécialistes du Vietnam, historiens de l'art, de la littérature, des sciences, archivistes.

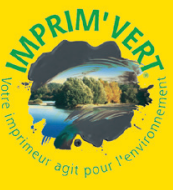

